Beate Hörr, Wolfgang Jütte (Hg.)

\title{
Weiterbildung an Hochschulen
}

\author{
Der Beitrag der DGWF zur Förderung \\ wissenschaftlicher Weiterbildung
}


Gesamtherstellung:

W. Bertelsmann Verlag, Bielefeld

wbv.de

Bielefeld 2017

Umschlagabbildung:

Robert Kneschke/Fotolia

Bestell Nr.: 6004479

ISBN: 978-3-7639-5564-0

DOI: $10.3278 / 6004479 w$

Printed in Germany
Diese Publikation ist frei verfügbar zum

Download unter wbv-open-access.de

Diese Publikation ist unter folgender CreativeCommons-Lizenz veröffentlicht:

http://creativecommons.org/licenses/by$\mathrm{sa} / 3.0 /$

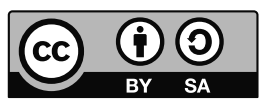

Für alle in diesem Werk verwendeten Warennamen sowie Firmen- und Markenbezeichnungen können Schutzrechte bestehen, auch wenn diese nicht als solche gekennzeichnet sind. Deren Verwendung in diesem Werk berechtigt nicht zu der Annahme, dass diese frei verfügbar seien.

\section{Bibliografische Information der Deutschen Nationalbibliothek}

Die Deutsche Nationalbibliothek verzeichnet diese Publikation in der Deutschen Nationalbibliografie; detaillierte bibliografische Daten sind im Internet über http://dnb.d-nb.de abrufbar. 


\section{Inhalt}

Geleitwort des Vorstandes der DGWF . . . . . . . . . . . . . . .

Der Beitrag der DGWF zur Förderung wissenschaftlicher Weiterbildung.

Zur Einführung

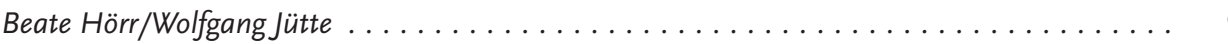

Zielsetzung und Wirkungsweise: die Fachgesellschaft $\ldots \ldots \ldots \ldots \ldots \ldots \ldots$

Entwicklung einer Fachgesellschaft: Die Deutsche Gesellschaft für wissenschaftliche Weiterbildung und Fernstudium e. V. im Wandel Beate Hörr . . . . . . . . . . . . . . . . . . . . . . . . .

Wissenskooperation durch Tagungen und Publikationen

Wolfgang Jütte/Claudia Lobe/Markus Walber

Thematische Bereiche: die Arbeitsgemeinschaften

Die Arbeitsgemeinschaft der Einrichtungen für Weiterbildung an Hochschulen (AG-E) Karla Kamps-Haller/Bernhard Christmann/Helmut Vogt

Zur Entstehungsgeschichte der Arbeitsgemeinschaft für das Fernstudium an Hochschulen (AG-F)

Burkhard Lehmann

Bundesarbeitsgemeinschaft Wissenschaftliche Weiterbildung für Ältere (BAG WiWA)

Thomas Bertram/Silvia Dabo-Cruz/Karin Pauls/Michael Vesper

Profilbildung und Professionalisierung durch Forschung. Die Arbeitsgemeinschaft Forschung

Wolfgang Jütte/Maria Kondratjuk/Mandy Schulze. 
Landesgruppe Baden-Württemberg

Ulrich Wacker

Landesgruppe Bayern

Gabriele Vierzigmann/Michael Renz/Sylvia Derra/Sybille Barth/Johannes Ries/Volker Stieg . . 101

Landesgruppe Berlin und Brandenburg

Peer-Olaf Kalis/Annette Strauß . . . . . . . . . . . . . . . . . . . . . . . . . . 109

Landesgruppe Hessen

Silke Vergara ....................................... 113

Landesgruppe Nord

Annekatrin Mordhorst/Sabine Riemer/Anno Stockem . . . . . . . . . . . . . . . . . . 117

Landesgruppe Nordrhein-Westfalen

Stefan Gesmann . . . . . . . . . . . . . . . . . . . . . . . . . . . . . . . 121

Landesgruppe Mitteldeutschland

Maria Kondratjuk/Kerstin Tänzer . . . . . . . . . . . . . . . . . . . . . . . . . . . . 127

Landesgruppe Rheinland-Pfalz und Saarland

Margot Klinkner .......................................... 133

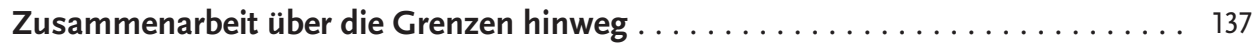

Netzwerk für wissenschaftliche Weiterbildung und Personalentwicklung der

Universitäten in Österreich: AUCEN

Elke Gornik/Monika Kil/Katharina Mallich-Pötz/Anna Steiger/Christine Stöckler-Penz

Wirkungsvolle Interessenvertretung der universitären Weiterbildung in der

Schweiz: Swissuni

Andreas Fischer

Working together to promote University Lifelong learning in Europe: eucen

Françoise de Viron 159 
Rechtliche und organisatorische Herausforderungen bei der Implementierung der wissenschaftlichen Weiterbildung

Ursula Bade-Becker

Offene Hochschule: Motor wissenschaftlicher Weiterbildung?

Andrä Wolter.

Beruflichkeit und wissenschaftliche Weiterbildung

Bernd Kaßebaum .

Zielgruppen in der wissenschaftlichen Weiterbildung

Wolfgang Seitter

Wissenschaftliche Weiterbildung im Medium von Praxisforschung eine relationstheoretische Deutung

Ortfried Schäffter

Empfehlungen der DGWF .

Organisation der wissenschaftlichen Weiterbildung an Hochschulen 243

Formate wissenschaftlicher Weiterbildung 255

Perspektiven wissenschaftlicher Weiterbildung in Deutschland aus Sicht der Einrichtungen an Hochschulen 263

Verzeichnis der Autorinnen und Autoren 279 



\section{Geleitwort des Vorstandes der DGWF}

Die Hochschullandschaft hat sich in den zurückliegenden Jahren erheblich verändert. Aus den akademischen Lehranstalten, die sich als Bildungsorte für die gesellschaftlichen Eliten verstanden haben, sind Hochschulen geworden, an denen sich mehr als $50 \%$ eines Jahrgangs auf die Wissensgesellschaft und die Wissensökonomie von morgen vorbereiten. Mahnende Stimmen wollen diesem vorgeblichen „Akademikerwahn“ Einhalt gebieten. Das zentrale Versprechen eines „Aufstiegs durch Bildung" hat jedoch bis heute nichts von seiner Sogwirkung eingebüßt. An die Stelle des traditionellen Diploms sind gestufte Studiengänge getreten, die eine Verknüpfung des Studiums mit Phasen der Berufstätigkeit ermöglichen, und Konzepte der offenen Hochschulen tragen zu mehr Chancengerechtigkeit bei, indem sie die Gleichwertigkeit verschiedenartiger Bildungsgänge betonen und für mehr Durchlässigkeit in einem durch Zugangsberechtigungen verregelten Bildungssystem sorgen.

Alle diese hier nur kursorisch angedeuteten Veränderungen des Hochschulwesens sind an der wissenschaftlichen Weiterbildung nicht spurlos vorbeigegangen: Ihr Weg, der in manchen Beschreibungen wie eine Art von Passionsgeschichte klingt, führt an den Stationen der "Göttinger Seminarkurse" vorbei, macht Halt beim Studium im dritten Lebensabschnitt oder einer „öffentlichen Wissenschaft“, um vorläufig bei den berufsbegleitend studierbaren Bachelor- oder den postgradual weiterbildenden Masterstudiengängen zu landen, von denen man Erstere im Windschatten der „offenen Hochschule“ auch ohne Abitur studieren kann. Angesichts solcher Entwicklungen und angesichts des respektablen Alters, das die „Deutsche Gesellschaft für Wissenschaftliche Weiterbildung und Fernstudium e. V.“ (DGWF) als Organisation erlangt hat, war es längst an der Zeit, Rechenschaft gegenüber sich selbst und der Fachöffentlichkeit abzulegen sowie eine Art von Inventur - oder besser Selbstvergewisserung - zu betreiben. Der drängende Impuls dazu hat sich Bahn gebrochen und herausgekommen ist ein umfangreiches Werk, dem Pioniercharakter zugeschrieben werden kann.

Der vorliegende Sammelband erfüllt mehrere Funktionen. Zum einen ist er Teil der Erinnerungsarbeit einer Fachgesellschaft. Aufgeschrieben wurde das, was bislang nur Teil eines kommunikativen Gedächtnisses war, das von den Akteurinnen und Akteuren bei verschiedenen Zusammenkünften immer wieder neu belebt und beschworen wurde, das sich nun aber mit dem fast vollzogenen Generationswechsel 
innerhalb der DGWF ausdünnt und gänzlich zu verschwinden droht. Zum anderen ist der Band Ausdruck des aktuellen Selbstverständnisses der Fachgesellschaft und liefert Dokumente und Materialien, um dieses zu fixieren. Aber nicht nur das: Die einzelnen Beiträge bieten erstmals eine Insidersicht auf die DGWF: Die Lebendigkeit der Organisation, ihre Gliederung in Landesgruppen und Sektionen sowie deren Wirken im Zusammenspiel mit der Arbeit des Vorstandes werden sichtbar. Das ist für alle von Bedeutung, die sich grundlegend über den Verband informieren möchten und/oder an einer potenziellen Mitwirkung interessiert sind. Der Sammelband bestimmt zudem die legitimatorischen Grundlagen der DGWF, auf deren Basis sie ihre Stimme für die wissenschaftliche Weiterbildung in all ihren Facetten erhebt, und er ist Teil eines umfassenden Kommunikationskonzeptes, das letztlich nur dazu dient, die Weiterbildung an Hochschulen weiter zu stärken, ihre Erfolge und ihre Bedeutung weithin sichtbar zu machen. Wozu auch sonst?

Ganz herzlicher Dank gilt Beate Hörr und Wolfgang Jütte. Ihrem unermüdlichen Engagement ist es zu verdanken, dass nun der erste umfassende Sammelband über die DGWF vorliegt. Der Dank gilt aber auch den vielen Autorinnen und Autoren, die an der Publikation mitgewirkt haben. Ohne sie wäre dem Vorhaben kein Erfolg beschieden gewesen.

Die Vorsitzenden

Burkhard Lehmann, Gabriele Vierzigmann, Silke Vergara 


\section{Der Beitrag der DGWF zur Förderung wissenschaftlicher Weiterbildung. Zur Einführung}

BEATE HÖRR/WOLFGANG JÜTTE

\section{Zielsetzung: Professionalisierung des Feldes}

Die Deutsche Gesellschaft für wissenschaftliche Weiterbildung und Fernstudium e. V. (DGWF) kann auf eine über 45-jährige Geschichte zurückblicken. Der vorliegende Band bilanziert die bisherige Arbeit der DGWF und entwirft Szenarien für die nahe Zukunft, verfolgt also eine doppelte Zielsetzung.

Zum einen will dieses Buch eine Zwischenbilanz über die Arbeit einer Fachgesellschaft ziehen. Dabei geht es jedoch nicht um eine umfassende historische Rekonstruktion. Weder ist die Geschichte der wissenschaftlichen Weiterbildung hinreichend aufgearbeitet worden, noch gibt es eine detaillierte Darstellung der DGWF. Punktuell wurde im Laufe ihrer Geschichte immer wieder ihre Arbeit reflektiert: Dies erfolgte entweder zum Anlass von Jubiläen - so zum 25-jährigen Bestehen (Dikau/Nerlich/Schäfer 1996) und zum 40-jährigen Bestehen (Schwerpunktheft „Hochschule und Weiterbildung“ 2010, H. 2) - oder im Rahmen einer Festschrift (Cordes/Dikau/Schäfer 2002). Eine chronologische Darstellung der Entwicklung der DGWF, deren zentrale Stationen, deren Pioniere und Promotoren, so wie von anderen wissenschaftlichen Fachgesellschaften vorgenommen (siehe für die Sektion Erwachsenenbildung Schmidt-Lauff 2014), steht ebenso noch aus wie eine Überführung der Empfehlungen, Papiere und Stellungnahmen in ein vollständiges digitales Archiv. Das eröffnet insofern noch die Möglichkeit der weiteren geschichtlichen Aufarbeitung zum 50. Geburtstag der DGWF. In dieser Zwischenbilanz werden anlassbezogen die strukturellen, systemischen und kontextuellen Bezüge beleuchtet, in denen die DGWF sich als Fachgesellschaft bewegt.

Zum anderen wird jedoch der Blick nach vorne gerichtet. Die strukturellen, systemischen und kontextuellen Bezüge werden auf die weitere Professionalisierung des Feldes bezogen. Dies ist vor dem Hintergrund des Wandels im letzten Jahrzehnt wie der starke Zuwachs an neuen Mitgliedern, die interne Ausdifferenzierung, ver- 
stärkte Kooperation mit anderen Fachgesellschaften (bspw. DGHD, DGfE, DGfHF), die Internationalisierung - ein wichtiger Entwicklungsschritt (siehe dazu auch den folgenden Beitrag in diesem Band). Die DGWF positioniert sich zunehmend als ein zentraler Akteur im Feld der Hochschulbildung.

\section{Community of Practice: miteinander lernen und gestalten}

Das vorliegende Buch gibt auch Einblick in die Verbandsarbeit und ihre Spannungsverhältnisse (siehe den Beitrag von Hörr in diesem Band). Ada Pellert hat in einem Beitrag zur dghd das Problem treffend charakterisiert: „[...] Wie intelligent agiert man organisatorisch, um etwa über Arbeitskreise und Delegation dezentrales Handeln zuzulassen und darauf zu achten, dass neue Themen und neue Kollegen und Kolleginnen hier ihren Platz finden. Partnerschaft und Delegation von Macht sind Erfolgsprinzipien funktionierender Netzwerke“ (Pellert 2013, S. 23). Eine Fachgesellschaft kann als eine Lern- und Wissensgemeinschaft verstanden werden.

Ein Kernstück der alltäglichen Arbeit der DGWF bilden neben der Arbeit im geschäftsführenden und Gesamtvorstand die ausdifferenzierten thematischen Arbeitsgemeinschaften und Landesgruppen. Ihre Beiträge spiegeln die thematische Bandbreite der Fachgesellschaft und ihre zahlreichen Vernetzungen wider. Dies eröffnet Einblicke in das „Verbandsleben“ und eröffnet Möglichkeiten des Engagements.

\section{Generationswechsel als Herausforderung}

Ein Blick in das vorliegende Verzeichnis der über 30 Autorinnen und Autoren im Anhang macht einen weiteren Wandel sichtbar. Während die Beiträge in den früheren Jubiläumsbänden überwiegend von Männern geschrieben worden sind, ist dies heute nicht mehr der Fall. Die Evaluation der Jahrestagungen (siehe dazu den Beitrag von Jütte/Lobe/Walber in diesem Band) zeigt, dass die Teilnehmenden zu etwa zwei Dritteln weiblich sind. Ohne hier näher auf die Gründe für diesen Wandel eingehen zu wollen - die Projektförmigkeit und Förderprogramme tragen sicherlich auch dazu bei -, erfährt die DGWF wie auch andere Organisationen neben der Erhöhung des Frauenanteils einen Generationswechsel. Als Bezugspunkte zu diesem Phänomen in der Weiterbildung führt Alke (2015, S. 113) an:

- „die Institutionalisierungs- und Professionalisierungsphase der Weiterbildung in den 1970er- und 1980er-Jahren, als dessen Folgeerscheinung der ,Generationenwechsel‘ zu erachten ist, sowie

- die spezifische Beschaffenheit von Weiterbildungseinrichtungen, die sich z. B. in ihrer Beziehungs- und Werteorientierung widerspiegelt, wodurch $u$. a. eine Affinität zu generationsbezogenen Diskursen in den Organisationen evoziert wird.“

Hier wird die Herausforderung für die Fachgesellschaft sichtbar. Die Älteren sind anders sozialisiert worden als viele Jüngere, die durch aktuelle Förderprogramme 
zur DGWF kommen; die Diskurse der 1970er-Jahre sind vielen der Jüngeren nicht mehr bekannt. Umso wichtiger, mit dieser Publikation einen Überblick über die DGWF-Arbeit und ihre Funktionsweisen auch für die neu hinzukommenden Mitglieder zu liefern.

\section{Wissenschaftliche Weiterbildung grenzenlos}

Internationale Bezüge spielen schon seit der Universitätsausdehnungsbewegung in der wissenschaftlichen Weiterbildung eine Rolle. Aber vor allem im letzten Jahrzehnt intensivieren sich die Kontakte auch vor dem Hintergrund der Europäisierung. Insofern ist es nur folgerichtig, dass hier auch AUCEN, SwissUni und eucen Einblicke in ihre Arbeit geben. Aber der grenzüberschreitende Austausch wird vor allem durch gemeinsame Jahrestagungen (etwa in Bern oder Wien) und die Arbeit in den Arbeitsgemeinschaften gelebt. Karl Weber hat in einem ausführlichen Interview (Weber 2016) auf die grenzüberschreitenden Lernprozesse verwiesen. „Sowohl der AUE wie die Sektion Erwachsenenbildung waren für mich wichtige Foren des Austausches, der wissenschaftlichen Diskussion, der Netzwerkbildung, einfach, weil sich diese Felder in Deutschland als relativ eigenständige Communities ausdifferenziert hatten.“ (ebd., S. 283). Dabei betonte er, dass bei der „Gestaltung [eigener] Weiterbildungsprojekte in den 1980er-Jahren der AUE mit seinen Publikationen eine wichtige Referenz darstellte“ (ebd., S. 276).

Die vorliegende Publikation wird als Open Access verbreitet. Die schnelle und freie Verbreitung relevanter Informationen gehört zunehmend zum Selbstverständnis einer modernen Fachgesellschaft. Die Website der DGWF (www.dgwf.net) eröffnet weitergehende Informationen zur Verbandsarbeit: zum aktuellen Vorstand', zur DGWF-Geschäftsstelle, zu den institutionellen Mitgliedern und gibt Hinweise auf aktuelle Veranstaltungen.

Der vorliegende Band eröffnet Interessierten an Fragen der wissenschaftlichen Weiterbildung die Möglichkeit, sich über Organisationsstrukturen, das Selbstverständnis, aktuelle Themen sowie die inhaltlichen Beiträge der DGWF in Form von Empfehlungen umfassend $\mathrm{zu}$ informieren und lädt zur Mitarbeit ein.

1 Auf der Jahrestagung 2016 wurden als neuer Vorsitzender Dr. Burkhard Lehmann, Universität Koblenz-Landau, und als stellvertretende Vorsitzende Dr. Silke Vergara, Universität Kassel, und Prof. Dr. Gabriele Vierzigmann, Hochschule für angewandte Wissenschaften München gewählt. 


\section{Literatur}

Alke, M. (2015): „Generationenwechsel“ in Weiterbildungseinrichtungen. Hessische Blätter für Volksbildung, (2), 106-115.

Cordes, M./Dikau, J./Schäfer, E. (Hrsg.) (2002): Hochschule als Raum lebensumspannender Bildung - Auf dem Weg zu einer neuen Lernkultur. Festschrift für Ernst Prokop.Regensburg.

Dikau, J./Nerlich, B. P./Schäfer, E. (Hrsg.) (1996): Der AUE an der Schnittstelle zwischen tertiärem und quartiärem Bildungsbereich: Bilanz und Perspektive; Festschrift aus Anlaß des 25-jährigen Bestehens des AUE. Bielefeld: AUE.

Pellert, A. (2013): Fachgesellschaften als Community of Practice: Lernen miteinander und Unterstützung füreinander. 40 Jahre dghd. In: Spiekermann, A. (Hrsg.): Lehrforschung wird Praxis. Hochschuldidaktische Forschungsergebnisse und ihre Integration in die Praxis. Bielefeld. 13-24.

Schmidt-Lauff, S. (Hrsg.) (2014): Vergangenheit als Gegenwart: zum 40-jährigen Bestehen der Sektion Erwachsenenbildung der DGfE. Opladen; Berlin.

Weber, K. (2016): Interview. Geführt von W. Jütte. In: Zimmermann, T. E./Jütte, W./ Horváth, F. (Hrsg.): Arenen der Weiterbildung. Bern, 269-291. 


\section{Zielsetzung und Wirkungsweise: die Fachgesellschaft}





\title{
Entwicklung einer Fachgesellschaft: Die Deutsche Gesellschaft für wissenschaftliche Weiterbildung und Fernstudium e.V. im Wandel
}

BEATE HÖRR

\begin{abstract}
Die DGWF blickt auf eine mehr als 45-jährige Geschichte zurück. Dies ist der Anlass die Entwicklung und den Wandel des AUE/der DGWF sowie ihre gewachsenen Strukturen zu beschreiben. Der Beitrag verortet außerdem die DGWF im nationalen, europäischen und internationalen Kontext. Es geht um den Beitrag der DGWF zu Fragen der Standortbestimmung und Definitionen der wissenschaftlichen Weiterbildung, veröffentlicht in ihrer Zeitschrift Hochschule und Weiterbildung, ihren Empfehlungen und Beiträgen.
\end{abstract}

\section{Die DGWF als Fachgesellschaft und die Stimme der wissenschaftlichen Weiterbildung in Deutschland}

Eine Fachgesellschaft ist eine Interessenvertretung und will inhaltlich und organisatorisch ein bestimmtes Thema in Politik und Gesellschaft vorantreiben. Die Deutsche Gesellschaft für wissenschaftliche Weiterbildung und Fernstudium e. V. hat sich vor mehr als 45 Jahren als Fachgesellschaft namens AUE (Arbeitskreis universitäre Erwachsenenbildung) in der Rechtsform eines gemeinnützigen Vereins gegründet. Der immer noch gültige und in der Satzung verankerte Gründungszweck war und ist die Förderung, Entwicklung, Koordinierung und Repräsentation der von den Hochschulen und hochschulnahen Einrichtungen getragenen wissenschaftlichen Weiterbildung und des Fernstudiums, und zwar nicht nur in Deutschland, sondern auch international. Das Ziel umfasst ausdrücklich die Förderung der Forschung und der Lehre sowie des wissenschaftlichen Nachwuchses auf dem Gebiet der Erwachsenenbildung und des Fernstudiums. Die DGWF will die wissenschaftliche Weiterbil- 
dung sichtbar machen und als die Stimme der wissenschaftlichen Weiterbildung Deutschlands gelten. Um repräsentativ zu sein, strebt sie eine möglichst hohe Zahl an Mitgliedern an. Diese Mitglieder können Hochschulen sowie hochschulnahe Einrichtungen sein oder auch Angehörige dieser Einrichtungen, die in der Hochschulweiterbildung tätig sind. Vor dem Hintergrund der sich ausdifferenzierenden Weiterbildungslandschaft sieht die Satzung der DGWF inzwischen auch die Mitgliedschaft von Institutionen oder Personen vor, die sich in Zusammenarbeit mit den Hochschulen um die Förderung der wissenschaftlichen Weiterbildung bemühen.

Die DGWF bietet ihren Mitgliedern ein Forum für den kollegialen Austausch auf ihren Jahrestagungen sowie den Veranstaltungen der Sektionen. Diese zwölf Sektionen der DGWF bestehen aus den vier thematischen Arbeitsgruppen (Arbeitsgemeinschaft der Einrichtungen für wissenschaftliche Weiterbildung an Hochschulen (AG-E), Arbeitsgemeinschaft für das Fernstudium (AG-F), Bundesarbeitsgemeinschaft wissenschaftliche Weiterbildung Älterer (BAG WiWa), Arbeitsgemeinschaft für Forschung sowie den acht Landesgruppen (Bayern, Baden-Württemberg, BerlinBrandenburg, Hessen, Mitteldeutschland, Nord, Nordrhein-Westfalen sowie Rheinland-Pfalz und Saarland).

Die DGWF setzt sich mittels Petitionen, Einlassungen und Berichten an einschlägige politische Stellen und Akteure dafür ein, dass die wissenschaftliche Weiterbildung gefördert wird. Der Mehrwert für die Mitglieder besteht darin, dass die DGWF als Verband ihre Interessen vertritt und eher Einfluss auf hochschulpolitische Entwicklungen zu nehmen vermag als die einzelne Einrichtung. Zwei aktuelle Beispiele für gelungene Interessenvertretung sind etwa die DGWF-Stellungnahme zum EUBeihilferecht sowie die Stellungnahme zur Änderung des Bundesstatistikgesetzes, die sich beide für die Interessen der wissenschaftlichen Weiterbildung positiv auswirkten.

\section{Das Dilemma - die DGWF zwischen Mitglieder-Logik und Einfluss-Logik}

Die DGWF als Fachgesellschaft ist ein Intermediär: Sie verbindet zwischen dem Wirken nach innen und nach außen. Die DGWF wirkt auf ihre Mitglieder, ihre Organe und Versammlungen einerseits („Mitglieder-Logik“) und strebt andererseits Sichtbarkeit in der Öffentlichkeit und politische Einflussnahme an („Einfluss-Logik“) (nach Hoffjann/Stahl 2010). Die DGWF bewegt sich zwischen den beiden Polen ihrer Professionspraxis sowie ihrem Fach einerseits und Politik und Öffentlichkeit andererseits. Sie fungiert als Dolmetscher: Sie übersetzt die eigenen Erkenntnisse und aus ihrer Sicht notwendigen Forderungen in politisch anschlussfähige und transportable Botschaften. Die DGWF erfüllt die Funktion eines Transmitters, indem sie auf Fachtagungen oder über verschiedene mediale Kanäle, sei es ihre Internetseite, ihre Fachzeitschrift „Hochschule und Weiterbildung“ (ZHWB) oder ihre regelmäßig ver- 
öffentlichten „Empfehlungen“ bzw. andere Publikationsformen, ihre Botschaft an relevante Akteure und die interessierte Öffentlichkeit sendet. Diese nach außen gerichteten Aktivitäten haben immer das Ziel Einfluss zu nehmen bzw. an Einfluss zu gewinnen. Diese Interessenvertretung ihrer Mitglieder ist ein entscheidender Bestandteil der Daseinsberechtigung der DGWF. Schließlich muss sie begründen können, warum man ihr beitreten sollte. Das Versprechen der Einflussnahme im Sinne des Mitgliederauftrags auf relevante Akteure spielt dabei eine wichtige Rolle.

Diese Scharnierfunktion der DGWF ließe sich auch mit negativem Vorzeichen beschreiben als „Sitzen zwischen den Stühlen“. Tatsächlich hat die DGWF wie alle Gesellschaften mit einem "Organisationsdilemma“ (vgl. Wiesenthal 1993) zu kämpfen. Auch innerhalb der DGWF herrscht dieser unauflösbare Widerspruch zwischen der Mitglieder-Logik einerseits und der Einfluss-Logik andererseits (Schmitter/Streeck 1981). Die DGWF strebt eine möglichst hohe Zahl an Mitgliedern an, sie will damit nach außen eine breite Unterstützung und Zustimmung signalisieren, ihre Legitimation und Glaubwürdigkeit erhöhen, ihr Einflusspotenzial sichern. Versucht die DGWF nun allen Mitgliedern in ihren hochschulpolitischen Forderungen gerecht zu werden, verwässern diese Forderungen in ihren Formulierungen zwangsläufig so sehr, dass sie hochschulpolitisch unwirksam sind. Versucht die DGWF durch eine Zuspitzung bzw. durch Komplexitätsreduktion der Mitgliedermeinung die Botschaft hochschulpolitisch verdaulich zu machen, verliert sie den Rückhalt ihrer Mitglieder, da diese sich nicht angemessen vertreten fühlen.

\section{Das Bild der DGWF in der Öffentlichkeit - Gelingens- faktoren einer modernen Verbandskommunikation}

Dieses Organisationsdilemma wirft auch ein Schlaglicht auf die überlebenswichtige Fähigkeit der DGWF als Intermediär: ihre Fähigkeit zur Kommunikation, und zwar nach innen (Mitglieder) und nach außen (Bildungs- und Hochschulpolitik, Öffentlichkeit). Heutzutage geht ohne eine erfolgreiche Kommunikationsarbeit auch in einer Fachgesellschaft nichts. Deshalb hat sich die DGWF entschieden ein umfassendes Kommunikationskonzept zu erarbeiten, das derzeit in der Phase der Implementierung ist.

Drei relevante Entwicklungen der Kommunikation sind zu beobachten und für die Arbeit der DGWF zu berücksichtigen: „Die Medialisierung insbesondere der politischen Kommunikation, die zurückgehende Bindung von Mitgliedern und die Pluralisierung organisierter Interessen." (Hoffjann/Stahl 2010, S. 59). Dieser Hintergrund des Bedeutungszuwachses der Verbandskommunikation bedeutet in der Konsequenz für die DGWF, dass die Anforderungen an ein professionelles Kommunikationsmanagement steigen (vgl. Seitter 2016, S. 118). Die Medialisierung erhöht den Druck auf die DGWF, die angesichts der entsprechenden Aktivitäten anderer konkurrierender Verbände mit diesen gleichziehen muss. 
Eine Professionalisierung von Kommunikationsmanagement an sich ist nicht das Problem, wenn die Mittel und die Fachleute da sind. Im Falle des ehrenamtlich arbeitenden DGWF-Vorstands stellt dies aber in der Tat ein großes Problem dar. Die organisationsstrukturellen Voraussetzungen sind alles andere als günstig. In der DGWF arbeiten keine Profis an Kommunikationskonzepten, sondern Laien, die wohl vom Gegenstand, nichts aber von dessen Vermarktung verstehen. Sich das teure Know-how auf dem Markt einzukaufen, erlauben die Finanzen der DGWF nicht. Um als kleinerer Verband in dem immer bunteren Markt der Mitgliedschaftsmöglichkeiten nicht unterzugehen, aber nicht selbst teure Werbekampagnen aus den knappen Ressourcen finanzieren zu müssen, sucht die DGWF bereits existente bundesweite oder europäische Plattformen, um diese zur Bekanntmachung des eigenen Verbandes zu nutzen. So hat sich die DGWF etwa 2016 entschlossen, als Kooperationspartner beim Deutschen Weiterbildungstag einzusteigen. Zwei Jahre lang ist damit die DGWF bundesweit Teil dieser Kampagne. Und eine ihrer Arbeitsgemeinschaften, die BAG WiWA sucht aus eben diesen Gründen seit einigen Jahren den engen Schulterschluss zur BAGSO, der Bundesarbeitsgemeinschaft der Seniorenorganisationen (siehe dazu auch den Beitrag zur BAG WiWA in diesem Band). Teil der Bemühungen der DGWF um eine zeitgemäße Kommunikation mit ihren Mitgliedern sowie mit anderen an der wissenschaftlichen Weiterbildung Interessierten ist die Revision der DGWF-Verbandszeitschrift „Hochschule und Weiterbildung“ (ZHWB) seit 2013. Diese erscheint wie bisher zweimal pro Jahr in gedruckter Form, ist aber mittlerweile, ebenso wie die Abstractbände zu den DGWF-Jahrestagungen, auch als Open Access erhältlich. Die Zeitschrift wurde hinsichtlich ihrer Rubriken umstrukturiert, es hat sich um den Herausgeber seitens des DGWF-Vorstands, Wolfgang Jütte, ein junges Redaktionsteam formiert und es wurden Formen des PeerReview-Verfahrens eingeführt, um die Qualität zu erhöhen.

\section{Zur Geschichte des AUE/der DGWF und der Entwicklung der Mitgliederstruktur}

Die Geschichte der wissenschaftlichen Weiterbildung (vgl. auch Schäfer 1988 und Dikau 2010) kann mit Wolter in drei Phasen eingeteilt werden, nämlich in „(1) der Universitätsausdehnungsbewegung (z. B. durch volkstümliche Hochschulkurse), etwa seit dem Ausgang des 19. Jahrhunderts, (2) der universitären Erwachsenenbildung, etwa seit der zweiten Hälfte der 1950er Jahre, und (3) der wissenschaftlichen Weiterbildung (seit Mitte der 1970er Jahre)“ (Wolter 2016, S. 23).

Die „Gesellschaft für Verbreitung von Volksbildung 1871“ war der erste deutschlandweit und inhaltlich umfassend agierende Volksbildungsverband. Die Gründung wissenschaftlicher Gesellschaften und Fachverbände im Deutschen Reich war zunächst aus einer Widerstandsbewegung entstanden: Gegen die innovationsfeindlichen, von Theologen und Philosophen dominierten Universitäten, aber auch gegen die Akademien (zur Geschichte wissenschaftlicher Gesellschaften vgl. Fröhlich 2002). Dieser 
Reformimpuls ist bis ans Ende des 20. Jahrhunderts der Beweggrund für Neugründungen geblieben. Die Neugründungen von Fachgesellschaften und Verbänden waren außerdem Ausdruck eines Ringens um Anerkennung kleinerer Fächer und Disziplinen. Die Erwachsenenbildung war als eigene Fachrichtung noch recht jung und die Professuren für Erwachsenenbildung waren noch nicht zahlreich (vgl. zur Geschichte der Erwachsenenbildung Seitter 2007, insbes. Kap. 7).

Auch die offizielle Gründung des „Arbeitskreises universitäre Erwachsenenbildung“ (AUE) entsprang diesem oben beschriebenen Reformimpuls und löste damals Unruhe bei den Etablierten aus: „Aber die erkennbare Reformeuphorie [...] hatte für viele zunächst auch was Beunruhigendes - für Universitätsvertreter, deren Interesse besonders auf die Erhaltung der bestehenden Hochschulstrukturen und Entscheidungsprozesse gerichtet war, sowieso, aber auch für stärker Reformwillige, denen solche Euphorie [...] den Rahmen des gesellschaftspolitischen Konsenses zu bedrohen schien“ (Dikau 2010, S.19).

Vom 04. bis o6. Januar 1968 fand an der Universität Göttingen die erste AUE-Jahrestagung statt zum Thema „Universität und Erwachsenenbildung“ mit Vorträgen der Gründungsväter Joachim Dikau, Hans-Dietrich Raapke, Hans-Dietrich Schmitz und Werner Schneider. Am 03. Oktober 1970 wurde im Rahmen der 3. AUE-Jahrestagung an der Technischen Universität Hannover die erste Mitgliederversammlung abgehalten, eine Satzung beraten und verabschiedet und Prof. Dr. Werner Schneider zum ersten Vorsitzenden des AUE gewählt. Auf dieser ersten Versammlung wurde ein 10-Punkte-Programm zur „Institutionalisierung der Erwachsenenbildung an den Universitäten der Bundesrepublik“ beschlossen. Es bot Universitäten den nötigen bildungspolitischen Rückenwind, um selbst auch Arbeitsstellen, „Kontaktstellen“ bzw. „Sekretariate für Seminarkurse“, wie die damaligen Einrichtungen für wissenschaftliche Weiterbildung hießen, zu gründen. Die erste Geschäftsstelle des AUE war ebenfalls in Hannover angesiedelt. Die Eintragung des AUE ins Vereinsregister beim Amtsgericht Hannover erfolgte drei Jahre später, am 20. Juli 1971. Im Jahr 2001 wurde der AUE umbenannt in „Deutsche Gesellschaft für wissenschaftliche Weiterbildung und Fernstudium e.V“, weil die wissenschaftliche Weiterbildung sich als Terminus etabliert hatte und auch das Fernstudium explizit als Teil der DGWF sichtbar werden sollte. Die 25-jährige Entwicklung des AUE wurde in einer eigenen Festschrift (Dikau/Nerlich/Schäfer 1996) gewürdigt, ebenso wie 40 Jahre nach Gründung ein Schwerpunktheft der Zeitschrift Hochschule \& Weiterbildung 2010/2 zur Entwicklung erschien, weshalb hier auf eine ausführliche Darstellung dieser Periode verzichtet wird.

Seit ihrer Gründung kennt die Entwicklung der Mitgliederzahlen der DGWF nur eine Richtung, nämlich nach oben. Die Zahl der DGWF-Mitglieder wächst insbesondere in den letzten zehn Jahren rasant. Allein in den letzten fünf Jahren ist die Zahl von 274 Mitgliedern in 2011 auf 319 in 2016 (Stand 01.01.2016) angewachsen. Gleichzeitig ist $\mathrm{zu}$ beobachten, dass die Mitgliedschaft heterogener geworden ist. Damit sind auch die Mitgliederinteressen, die es zu bedienen gilt, vielfältiger geworden 
und gleichzeitig sind damit die Ansprüche der Mitglieder gewachsen. Das ist ein Trend, der nicht nur die DGWF, sondern viele Fachgesellschaften betrifft (vgl. Steiner/Jarren 2009, S. 260). Außerdem sind die Mitglieder mittlerweile selbstbewusster und verstehen sich auch als „Kunden“ der DGWF. Die Ansprüche an den Service der DGWF sind ebenfalls gestiegen, wie $u$. a. die wachsende Zahl an Anfragen an die Geschäftsstelle der DGWF beweist.

Ein Ausdruck dieses neuen Selbstbewusstseins der Mitglieder und ihres veränderten Anspruchsdenkens ist die - absolut berechtigte - Forderung nach mehr Transparenz in der Kommunikation nach innen, etwa bei der Kandidatensuche für neu zu besetzende DGWF-Vorstandsposten und den Wahlverfahren. Auch in der DGWF sind die Zeiten vorbei, in denen informelle Zirkel an den Abenden vor der Wahl diese am Kneipentisch schon einmal vorbereitete und entschied. Tatsächlich wurden seit der Gründung im Jahr 1970 die Geschicke der DGWF bis 2012 im geschäftsführenden Vorstand ausschließlich von Männern gelenkt. Im Gesamtvorstand der DGWF gab es jahrelang nur eine Frau, erst ab 2003 kamen nach und nach weitere Frauen dazu. Mittlerweile ist der DGWF-Vorstand des Jahres 2016 mit 50\% Frauen und mit 50\% Männern besetzt. Im geschäftsführenden Vorstand haben sogar mittlerweile (seit 2012) die Frauen die Mehrheit (zwei Frauen, ein Mann).

Mehr Basisdemokratie ist ein weiteres, ebenfalls vollkommen berechtigtes und nachvollziehbares Anliegen der „neuen“ Mitglieder. Wie Evaluationen der letzten DGWFJahrestagungen (siehe dazu auch den Beitrag in diesem Band) durchgängig zeigen, hat sich an der Hauptmotivation einer Mitgliedschaft jedoch nichts geändert: Im Mittelpunkt steht nach wie vor der fachliche und persönliche Austausch, sei es im Rahmen von Tagungen oder Landes- und Arbeitsgruppentreffen, trotz oder vielleicht gerade in Zeiten von social media.

\section{Struktur der DGWF}

Die DGWF ist ein gemeinnütziger Verein, alle Vorstandsmitglieder arbeiten ehrenamtlich. Aufgrund der stark angewachsenen Mitgliederzahl wird die Geschäftsstelle der DGWF seit zwei Jahren durch eine bei der DGWF angestellte Assistenz unterstützt. Die DGWF finanziert sich weitgehend aus Mitgliedsbeiträgen, diese liegen bei derzeit $330 €$ pro Jahr für institutionelle Mitglieder. Hinzu kommen die Einnahmen aus Tagungen, sofern Überschüsse erzielt werden. Die Mitgliederstruktur setzt sich aus etwa zwei Drittel Institutionen und einem Drittel persönlicher Mitglieder zusammen.

\subsection{Beirat}

Die DGWF lässt sich von einem Beirat in grundsätzlichen Fragen der wissenschaftlichen Weiterbildung beraten. Die Mitglieder des Beirats sind Vertreterinnen und Vertreter von Organisationen, die für die wissenschaftliche Weiterbildung von Bedeu- 
tung sind, sowie Angehörige anderer überregionaler Erwachsenenbildungs- oder Forschungseinrichtungen. Derzeit setzt sich der Beirat aus Vertreterinnen und Vertretern des Bundesministeriums für Bildung und Forschung (BMBF), der Hochschulrektorenkonferenz (HRK), der Gewerkschaft (IG Metall), des Deutschen Instituts für Erwachsenenbildung (DIE), des Bundes Deutscher Arbeitgeber (BDA), des Volkshochschulverbands (vvhs), der Kultusministerkonferenz (KMK) sowie aus zwei Hochschulvertreterinnen und Hochschulvertretern zusammen. Der Beirat kommt zu einer halbtägigen Sitzung gemeinsam mit dem Gesamtvorstand der DGWF einmal pro Jahr zusammen.

\subsection{Beisitzer}

Die vier Beisitzer sind Mitglieder des DGWF-Vorstandes und werden wie der geschäftsführende Vorstand auf zwei Jahre von der Mitgliederversammlung gewählt. Die Beisitzer haben bestimmte inhaltliche Zuständigkeiten (etwa die Ressorts „Tagungen“, „Qualitätsmanagement“, „Kommunikation“, „Internationales“), in denen sie den Vorstand unterstützen und ihm zuarbeiten.

\subsection{Sektionen (Arbeitsgemeinschaften und Landesgruppen)}

Die DGWF ist in ihrer heutigen Struktur stark ausdifferenziert, sowohl in thematischer als auch regionaler Hinsicht. Es gibt mittlerweile vier Arbeitsgemeinschaften und acht Landesgruppen, in denen alle 16 Bundesländer vertreten sind. Die Genese, die Arbeitsschwerpunkte und Herausforderungen dieser zwölf Sektionen sind in den jeweiligen Kapiteln ausführlich beschrieben, auch hinsichtlich ihrer historischen Entwicklung. Sie geben einen vertiefenden Einblick in die Arbeit der DGWF.

\subsubsection{Arbeitsgemeinschaften}

Die Ausdifferenzierung in thematische Arbeitsgruppen begann bereits früh im AUE. Schon 1971 wurden erste AUE-,Kommissionen“ eingerichtet, die sich den verschiedenen zu behandelnden Themen widmen sollten. Es entstanden zuerst die Kommissionen „Weiterbildung der Studiengänge/Mitarbeit in der Erwachsenenbildung“ sowie „Kontaktstudium“ und „Kontaktstellenkonzeption“ (heutige Zentren für wissenschaftliche Weiterbildung). Hieraus entwickelte sich die Arbeit der ersten Arbeitsgemeinschaft der DGWF, der Arbeitsgemeinschaft für Einrichtungen (AG-E). Sie begann ihre Arbeit 1981 mit einer Tagung in Kassel als Vorbereitung zu einem Projekt. In der AG-E geht es um Fragen der Organisation, aber auch um das eigene Professionsverständnis. Außerdem formierte sich 1979 die BAG WiWA, wenn auch noch nicht als Arbeitsgemeinschaft der DGWF (das geschah erst 1994), sondern zunächst als loser Zusammenschluss von Hochschulen, die sich in diesem Bereich engagierten. Ein Jahr nach Gründung der BAG WiWA, also 1995, erfolgte die Gründung der Arbeitsgemeinschaft Fernstudium, einer zunächst eigenständigen Gruppierung bestehend aus Vertretern der Studienzentren der Fernuniversität Hagen, die dann in der DGWF als dritte Arbeitsgemeinschaft aufging. Erst 2012 kam die Ar- 
beitsgemeinschaft Forschung als jüngste Arbeitsgemeinschaft hinzu. Diese Arbeitsgemeinschaft trägt der Tatsache Rechnung, dass es bis dahin für die Nachwuchswissenschaftlerinnen und -wissenschaftler kein eigenes Forum für den Austausch über die wissenschaftliche Weiterbildung gab und dass die DGWF dezidiert den Schulterschluss zum wissenschaftlichen Bereich der Erwachsenenbildung und Weiterbildung suchte.

Die thematischen Arbeitsgemeinschaften innerhalb der DGWF sind Orte des Austauschs zwischen den Mitgliedern entlang ihrer eigenen Arbeitsschwerpunkte und Interessen. Sobald eine Einrichtung oder eine Person der DGWF beitritt, kann sie entscheiden, in welcher Arbeitsgemeinschaft und/oder Landesgruppe sie mitarbeiten möchte. Sowohl die Arbeitsgemeinschaften als auch die Landesgruppen halten regelmäßige Treffen ab. Alle Arbeitsgemeinschaften bieten im Frühjahr eine Tagung an, die Landesgruppen treffen sich regelmäßig zu Sitzungen, häufig auch mit geladenen Gästen, um die Kooperationen vor Ort mit relevanten Akteuren der Weiterbildung, mit Unternehmen und Ministerien zu stärken.

\subsubsection{Landesgruppen}

Die Landesgruppen haben insofern eine besondere Bedeutung für die Arbeit der DGWF, als die gesetzlichen Bestimmungen zur wissenschaftlichen Weiterbildung in den jeweiligen Hochschulgesetzen, die Landesgesetze sind, geregelt werden. Während die DGWF als Gesamtorganisation zwar auf Ebene des Bundes agieren kann, wird die Hochschulpolitik in den Ländern gemacht. Diese Länderbestimmungen prägen die Tagespolitik und die Rahmenbedingungen der Kolleginnen und Kollegen, die im Bereich der wissenschaftlichen Weiterbildung an Hochschulen arbeiten. Um also angemessen auf die sich aus diesen konkreten Landesgesetzen möglicherweise ergebenden Probleme und Fragestellungen der wissenschaftlichen Weiterbildung reagieren zu können, sind konzertierte Aktionen als Landesgruppe der DGWF etwa gegenüber dem zuständigen Ministerium hilfreich. Der kollegiale Austausch vor allem mit denjenigen, die unter denselben Rahmenbedingungen arbeiten, ist äußerst hilfreich. Deshalb hat sich der Vorstand der DGWF seit 2012 gezielt darangemacht, die noch fehlenden Landesgruppen zu gründen, sodass seit 2015 alle Bundesländer in DGWF-Landesgruppen vertreten sind. Um eine bessere Verzahnung zwischen der Arbeit in den Landesgruppen und der Ausrichtung der DGWF insgesamt zu erreichen, haben mittlerweile die jeweiligen Sprecherinnen und Sprecher der Landesgruppen, ebenso wie schon bisher die Sprecherinnen und Sprecher der Arbeitsgemeinschaften, einen Sitz im DGWF-Vorstand. Auf diese Weise soll die Abstimmung zwischen der Arbeit in allen Sektionen und der Arbeit des übrigen Vorstandes verbessert werden. Damit wuchs der DGWF-Vorstand in der Amtsperiode 2012 bis 2016 auf insgesamt 18 Personen an. 


\section{Die DGWF im Dialog: Kooperationen}

Der AUE vernetzte sich von Anfang an strategisch mit Verbänden, Institutionen und Einrichtungen, die er für die Weiterentwicklung der wissenschaftlichen Weiterbildung für wichtig erachtete. Gleich nach seiner Gründung am 6. Oktober 1971 wurde vom AUE ein Beirat eingesetzt, in diesem saßen Vertreterinnen und Vertreter des damaligen Bundesbildungsministeriums, der KMK, der WRK, des BDA, des DGB sowie von ARD und ZDF. Der AUE leistete auch die Geburtshilfe für eine neue Sektion der Deutschen Gesellschaft für Erziehungswissenschaft (DGfE): Im Anschluss an die AUE-Jahrestagung am 9. Oktober 1971 fand die konstituierende Sitzung der „Sektion Erwachsenenbildung“ der Deutschen Gesellschaft für Erziehungswissenschaft statt. Die Fachvertreter des AUE hatten in beiden Gesellschaften eine Mitgliedschaft inne, was sich günstig auf die enge inhaltliche Verzahnung auswirkte (Dikau 2002, S.20). Der Deutsche Verband der Volkshochschulen (DVV) hatte seit der Gründung des AUE lange Jahre einen Vorstandsposten im AUE als geborenes Mitglied inne. Der Schulterschluss mit anderen freien Trägern der Erwachsenenbildung wurde vom AUE bewusst von Anfang an gesucht. Dieser von der Gründung des AUE an sichtbare Kooperationswillen ist auch in der DGWF erhalten geblieben. Die DGWF arbeitet bis heute eng mit der DGfE, der Hochschulrektorenkonferenz (HRK), dem Deutschen Volkshochschulverband e. V. (DVV), der Deutschen Gesellschaft für Hochschulpädagogik (DGHD), dem Deutschen Institut für Erwachsenenbildung (DIE) ${ }^{7}$, Gewerkschaften, Stiftungen und Verbänden bzw. Kammern wie dem BDA und dem DIHT zusammen, um nur die wichtigsten zu nennen.

Die DGWF kooperiert seit ihrer Gründung mit dem Bundesministerium für Bildung und Forschung. Bereits 1975 brachte der AUE gemeinsam mit dem damaligen BMBW das Fördervorhaben „Öffnung der Hochschulen durch den Ausbau der wissenschaftlichen Weiterbildung im Zusammenwirken von Hochschulen und Weiterbildungseinrichtungen des Einzugsbereichs“ auf den Weg. Auch in die Planung und Durchführung des im Jahr 2011 vom BMBF gestarteten Bund-Länder-Wettbewerbs „Aufstieg durch Bildung. Offene Hochschulen“ war die DGWF eingebunden. Dieser Wettbewerb hat noch einmal deutlich Dynamik in den Bereich der wissenschaftlichen Weiterbildung gebracht. Es bleibt - wie bei allen Projektförderungen - abzuwarten, welche Bilanz insbesondere im Hinblick auf die Nachhaltigkeit nach Auslaufen der Förderung zu ziehen sein wird.

1 Aktuell ist hier besonders die Kooperation im Rahmen des laufenden Projekts GRETA zur Professionalisierung von Erwachsenenbildnern zu erwähnen. 


\section{Die DGWF im internationalen Kontext des University Lifelong Learning (ULLL)}

Die Tradition der internationalen Ausrichtung der DGWF reicht bis in ihre Anfänge zurück. Der AUE trat bereits im November 1971 dem „International Congress of Universities for Adult Education“ bei. Der AUE blieb stets in gutem Kontakt zu den einschlägigen internationalen Verbänden und Einrichtungen. Seit Ende der 9oer-Jahre kümmerte sich die damalige Vizepräsidentin der Universität Oldenburg, Ina Grieb, u. a. zuständig für Weiterbildung, im DGWF-Vorstand als Beisitzerin um das Ressort „Internationales“.

Die DGWF ist bis heute auf europäischer und internationaler Ebene gut vernetzt. So ist ein Beisitzer im DGWF-Vorstand Mitglied des Schweizer Netzwerks für wissenschaftliche Weiterbildung an Universitäten, swissuni (vgl. dazu den Beitrag von Andreas Fischer). Zwei Jahrestagungen der DGWF fanden bereits in Kooperation mit dem österreichischen Verband für wissenschaftliche Weiterbildung, AUCEN (Austrian Universities Continuing Education Network), an der Universität Wien statt (2005 und 2016). Und schließlich pflegt die DGWF seit vielen Jahren enge Beziehungen zu dem europäischen Verband der wissenschaftlichen Weiterbildung an Universitäten, EUCEN (European Universities Continuing Education). Im Jahr 2007 fand eine EUCEN-Jahrestagung an der Universität Hannover statt, in 2017 wird die Universität Mainz Gastgeberin sein. Einzelne Mitglieder der DGWF haben sich auch innerhalb des EUCEN-Vorstandes engagiert, sie haben Mandate für die Vertretung in anderen Netzwerken wie EUCIS LLL oder bei Hearings bei der Kommission in Brüssel übernommen (siehe zu EUCEN auch den Beitrag in diesem Band von Francoise de Viron, der derzeitigen EUCEN-Präsidentin). Auch mit den Netzwerken in Lateinamerika (RECLA, Red de Educación Contínua Latinoamérica) und in den USA (UPCEA, University Professional and Continuing Education Association) steht die DGWF mittlerweile in Kontakt.

Der Aufgabenzuschnitt der verschiedenen internationalen Gesellschaften ist dabei unterschiedlich: Während die DGWF, swissuni, EUCEN und RECLA sich ausschließlich um die wissenschaftliche Weiterbildung an Hochschulen kümmern, befassen sich die Verbände AUCEN und UPCEA auch um die Belange der Personalfortbildung an Hochschulen.

Insbesondere aufgrund der Entwicklungen im Bereich E-Learning und Fernstudium, die es ermöglichen Lernen zeit- und ortsunabhängig zu machen, ist eine verstärkte Kooperation der verschiedenen Akteure auf internationaler Ebene sinnvoll. Die Zusammenarbeit ist aber auch aus Sicht der Organisationsentwicklung wichtig. Wenn Einrichtungen für wissenschaftliche Weiterbildung die Gelegenheit nutzen und im Rahmen etwa von europäischen Projekten zusammenarbeiten, dann profitieren nicht nur das jeweilige Programm oder bestimmte Themen davon, sondern auch die Organisation selbst. Der Mehrwert liegt in dem Voneinanderlernen und dem Kennenlernen von Beispielen guter Praxis. Und nicht zuletzt erfordert der eu- 
ropäische Rechtsrahmen einen Blick über den eigenen nationalen Tellerrand. So hat etwa der beihilferechtliche EU-Rahmen in einigen Einrichtungen bereits konkrete Auswirkungen auf die Kalkulationsmodelle der wissenschaftlichen Weiterbildung. Neben allen Gemeinsamkeiten, die im Dialog mit Kolleginnen und Kollegen aus anderen Ländern festzustellen sind, stößt das begriffliche Verständnis dessen, was unter "wissenschaftlicher Weiterbildung“ in Deutschland zu verstehen ist, im Ausland (Ausnahmen bilden Österreich und die Schweiz) eher auf Unverständnis.

\section{Definitionen, Empfehlungen und Beiträge der DGWF}

Bewegt man sich auf internationalem Parkett, dann ist es schwierig zu erklären, was der deutsche Begriff „wissenschaftliche Weiterbildung“ genau umfasst und wie er zu verstehen ist. Es gibt dazu verschiedene Definitionen und Ansätze, sowohl im deutschen als auch europäischen bzw. internationalen Diskurs. ${ }^{2}$ Aber zunächst einmal kann festgehalten werden, dass das Attribut „wissenschaftlich“ auf die institutionelle Herkunft verweist: Anbieter der wissenschaftlichen Weiterbildung sind wissenschaftliche Einrichtungen, also Hochschulen oder hochschulnahe Einrichtungen. Das impliziert, dass es sich beim Lehrpersonal um Wissenschaftlerinnen und Wissenschaftler handelt. Das Attribut „wissenschaftlich“ bezieht sich außerdem auf das Niveau: Es werden Programme angeboten, die forschungsbasiert und auf dem neuesten Stand der Erkenntnisse sind, gleichzeitig aber einen engen Bezug zur Praxis haben sollen. ${ }^{3}$

\subsection{Definitionen}

Das Konzept des Lebenslangen Lernens (LLL) wurde im Zuge der europäischen Beschäftigungsstrategie definiert als „alles Lernen während des gesamten Lebens, das der Verbesserung von Wissen, Qualifikationen und Kompetenzen dient und im Rahmen einer persönlichen, bürgergesellschaftlichen, sozialen, bzw. beschäftigungsbezogenen Perspektive erfolgt“. (Europäische Kommission 2001, S. 34). In Deutschland wird bis heute das aus 2001 stammende Verständnis der wissenschaftlichen Weiterbildung bemüht, wie es die KMK formulierte. Hiernach wird Weiterbildung an Hochschulen definiert als „die Fortsetzung oder Wiederaufnahme organisierten Lernens nach Abschluss einer ersten Bildungsphase und in der Regel nach Aufnahme einer Erwerbs- oder Familientätigkeit, wobei das wahrgenommene Weiterbildungsangebot dem fachlichen und didaktischen Niveau der Hochschule entspricht. [...] Wissenschaftliche Weiterbildung knüpft in der Regel an berufliche Erfahrungen

2 Zu nennen sind hier etwa die EUA Charter on LLL, die Definitionen der 17 Nationalen Netzwerke, die in EUCEN Mitglied sind, sowie etwa die Veröffentlichungen Slowey/Schuetze 2013 oder Hanft/Knust 2007. Aktuellste Annäherung an die Begriffe Lebenslanges Lernen und akademische Bildung: Banscherus/Wolter 2016, S. $53 \mathrm{ff}$.

3 Wolter (2016, S. 24) nennt noch die Zielgruppen als kennzeichnend für das Attribut „wissenschaftlich“: „Weiterbildung von Hochschulabsolvent_innen“, fügt aber direkt einschränkend hinzu: „... (auch wenn viele Maßnahmen für Personen ohne Hochschulabschluss offen sind)." 
an, setzt aber nicht notwendigerweise einen Hochschulabschluss voraus “. (KMK 2001, S. 2 f.). Im Zuge der Öffnung der Hochschulen für beruflich Qualifizierte haben sich die Begriffe und Herleitungen insbesondere unter Einbezug der internationalen Perspektive verändert. In den letzten Jahren wird der Begriff des „University Lifelong Learning“ (ULLL) auch im deutschsprachigen Diskurs gerne synonym verwendet für die wissenschaftliche Weiterbildung. Außerdem wird versucht keine Definition des Begriffs, sondern unterschiedliche Arten des Verständnisses zu liefern. So beschreiben etwa Wolter/Banscherus (2016) basierend auf dem Konzept des lebenslangen Lernens vier verschiedene Ansätze in Hochschulen zur wissenschaftlichen Weiterbildung (Inklusions-Ansatz, holistischer Ansatz, Delivery-Ansatz und Arbeitsmarktansatz, ebd. S. 68 ff.). An der wissenschaftlichen Behandlung der Frage der wissenschaftlichen Weiterbildung in der letzten Dekade lässt sich gut ablesen, dass die Betrachtung zwar mittlerweile sehr ausdifferenziert ist, dass aber eine aktuelle Definition der wissenschaftlichen Weiterbildung fehlt bzw. die Behandlung der Frage, ob denn dieser Begriff immer noch zu verwenden sei und wenn ja mit welcher Bedeutung, noch offen ist.

\subsection{Empfehlungen und Beiträge}

Die DGWF selbst hat im Laufe ihrer mehr als 45-jährigen Geschichte zahlreiche Empfehlungen herausgegeben und damit einen wichtigen Beitrag zur hochschulpolitischen und wissenschaftlichen Diskussion geleistet.

Bereits 1975 veröffentlichte der AUE „Materialien zur Studien- und Berufssituation in der Erwachsenenbildung: Gesamterhebung“. 1980, also nach einem Jahrzehnt AUE, erschienen vier Empfehlungen des AUE zu den Weiterbildungsaufgaben der Hochschulen: die „Empfehlungen zum weiterbildenden Studium“, die „Empfehlungen zur Beteiligung der Hochschulen an Veranstaltungen der Weiterbildung“, die „Empfehlungen zur Weiterbildung des Hochschulpersonals“ und die „Empfehlungen zu den Studiengängen Weiterbildung“. Die jüngsten Empfehlungen der DGWF befassen sich mit Fragen der Organisation und der Formate. Alle Empfehlungen sind über die DGWF-Seite abrufbar.

Abgesehen von den Empfehlungen haben immer wieder Mitglieder insbesondere des geschäftsführenden DGWF-Vorstandes eigene Beiträge zur wissenschaftlichen Weiterbildung und deren Selbstverständnis verfasst. So hat etwa der frühere DGWFVorsitzende Peter Faulstich sich insbesondere mit wissenschaftlichen Beiträgen zum Thema der öffentlichen Wissenschaft befasst (bspw. Faulstich 2008). Der bisherige stellvertretende Vorsitzende der DGWF, Andrä Wolter, Hochschulforscher an der Humboldt-Universität zu Berlin, hat $u$.a. in seiner Funktion als einer der wissenschaftlichen Begleiter des Projekts der „Offenen Hochschule“, aber auch als Mitglied in der Kommission, die für den Nationalen Bildungsbericht zuständig ist, zahlreiche Veröffentlichungen zu Fragen der wissenschaftlichen Weiterbildung beigesteuert. Diese Tradition der engen Verknüpfung zwischen Wissenschaftsbezug einerseits 
und organisationspraktischen Fragen andererseits ist bis heute ein wesentliches Merkmal der DGWF-Arbeit geblieben.

\section{Ausblick}

Die DGWF hat in ihrer über 45-jährigen Geschichte als Fachgesellschaft u.a. über ihre Veröffentlichungen viele wichtige Impulse sowohl in die Hochschulen hinein, als auch in die hochschulpolitische Debatte zu Fragen der wissenschaftlichen Weiterbildung geliefert. Sie hat nicht selten als Referenzpunkt gedient, wenn es darum ging, an Hochschulen die wissenschaftliche Weiterbildung zu etablieren oder neu zu strukturieren. Die DGWF ist mittlerweile allein zahlenmäßig ein „Schwergewicht“ unter den Fachverbänden der Hochschullandschaft. Sie ist gut vernetzt und zieht mit ihren renommierten Jahrestagungen mittlerweile weit über 300 Gäste an - auch aus den europäischen Nachbarländern. Wohin kann also die Reise der DGWF noch gehen, so könnte man fragen?

$\mathrm{Zu}$ den Fragen, die immer wieder aufkommen und die bis heute nicht befriedigend oder abschließend geklärt sind, gehört die Frage nach der Begrifflichkeit und Definition der wissenschaftlichen Weiterbildung in Zeiten von Internationalisierung und gestuften Studiengängen mit Bachelor- und Master-Abschlüssen. Des Weiteren gehört dazu die Frage der Finanzierung der wissenschaftlichen Weiterbildung, die eng zusammenhängt mit der Frage nach dem Verhältnis zwischen staatlich und privat finanzierten Hochschulen und Bildungsangeboten. Auch die Fragen nach geeigneten Formaten der wissenschaftlichen Weiterbildung und ihren didaktisch-methodischen Implikationen und Voraussetzungen ist auch bzw. gerade in Zeiten von MOOC und E-Learning drängend. Der DGWF gehen also auch nach über 45 Jahren Diskussion und Ringen um Fragen der wissenschaftlichen Weiterbildung und des Fernstudiums in Deutschland drängende und zu behandelnde Themen nicht aus.

\section{Literatur}

Dikau, J./Nerlich, B./Schäfer, E. (Hrsg.) (1996): Der AUE an der Schnittstelle zwischen tertiärem und quartärem Bildungsbereich. Bilanz und Perspektive. Festschrift zum 25jährigen Bestehen. Bielefeld.

Dikau, J. (2002): Wissenschaftliche Weiterbildung an Hochschulen in Berlin und Brandenburg. Eine Bestandsaufnahme. In: Informationsdienst Hochschule \& Weiterbildung, AUE Sonderheft 2002.

Dikau, J. (2010): Die Frühphase der universitären Erwachsenenbildung in der Bundesrepublik Deutschland Von ihren ersten Ansätzen bis zur Bildungsreform der siebziger Jahre. In: Hochschule \& Weiterbildung, H. 2, S. 16-25. 
Europäische Kommission (Hrsg.) (2001): Einen europäischen Rahmen des lebenslangen Lernens schaffen. Mitteilung der Kommission KOM (2001) 678 vom 21.11.2001. https:// www.bibb.de/dokumente/pdf/foko6_neues-aus-euopa_o4_raum-lll.pdf

Faulstich, P. (2008): Vermittler wissenschaftlichen Wissens: Biographien von Pionieren öffentlicher Wissenschaft. Münster.

Fröhlich, G. (2002): Verein.Wissenschaft. Entstehung und Funktion wissenschaftlicher Gesellschaften. In: Kammerhofer-Aggermann, U. (Hrsg.): Ehrenamt und Leidenschaft. Vereine als gesellschaftliche Faktoren. Salzburger Landesinstitut für Volkskunde, S. 255-278.

Hanft, A./Knust, M. (2007): Weiterbildung und lebenslanges Lernen an Hochschulen. Eine internationale Vergleichsstudie zu Strukturen, Organisation und Angebotsformen. Münster.

Hoffjann, O./Stahl, R. (Hrsg.) (2010): Handbuch Verbandskommunikation. Wiesbaden: Springer Verlag.

KMK (Konferenz der Kultusminister der Länder in der Bundesrepublik Deutschland) (2001): Sachstands- und Problembericht zur „Wahrnehmung wissenschaftlicher Weiterbildung an Hochschulen“. Beschluss der Kultusministerkonferenz vom 21.09.2001. http:// www.kmk.org/fileadmin/Dateien/veroeffentlichungen_beschluesse/2001/2001_09_21Problembericht-wiss-Weiterbildung-HS.pdf

Schäfer, E. (1988): Historische Vorläufer der wissenschaftlichen Weiterbildung. Von der Universitätsausdehnungsbewegung bis zu den Anfängen der universitären Erwachsenenbildung in der Bundesrepublik Deutschland. Opladen.

Schmitter, P./Streeck W. (1981): The Organization of Business Interests: Studying the Associative Action of Business in Advanced Industrial Societies. MPIfG Discussion Paper, IIM/LMP, S. 81-13.

Seitter, W. (2007): Geschichte der Erwachsenenbildung. Eine Einführung. Bielefeld: W. Bertelsmann Verlag.

Seitter, W. (2016): Herausforderungen und Möglichkeitsräume eines intermediär verorteten Verbandes. In: Hessische Blätter für Volksbildung: 70 Jahre hvv - Verbände und Supportstrukturen, 2, S. 116-123.

Slowey, M./Schuetze, H. (2013): Global perspectives on higher education and lifelong learners. Routledge paperback.

Steiner, A./Jarren, O. (2009): Intermediäre Organisationen unter Medieneinfluss? Zum Wandel der politischen Kommunikation von Parteien, Verbänden und Bewegungen. In: Marcinkowski, F./Pfetsch, B. (Hrsg.): Politik in der Mediendemokratie. Wiesbaden: Springer Verlag, S. 251-269.

Wiesenthal, H. (1993): Akteurkompetenz im Organisationsdilemma. Grundprobleme strategisch ambitionierter Mitgliederverbände und zwei Techniken ihrer Überwindung. In: Berliner Journal für Soziologie 3 (1), S. 3-18.

Wolter, A. (2016): Die Rolle der Hochschulen auf dem Weiterbildungsmarkt. In: Borgwardt, A.: Akademische Weiterbildung. Eine Zukunftsaufgabe für Hochschulen. Schriftenreihe Hochschulpolitik (Friedrich-Ebert-Stiftung), Berlin, S. 23-36. 
Wolter, A./Banscherus, U. (2016): Lebenslanges Lernen und akademische Bildung als Reformprojekt. In: Wolter, A./Banscherus, U./Kamm, C. (Hrsg.): Zielgruppen Lebenslangen Lernens an Hochschulen, Ergebnisse der wissenschaftlichen Begleitung des BundLänder-Wettbewerbs Aufstieg durch Bildung: offene Hochschulen. Band 1. Münster, S. 53-80.

Wolter, A./Banscherus, U./Kamm, C. (Hrsg.) (2016): Zielgruppen Lebenslangen Lernens an Hochschulen, Ergebnisse der wissenschaftlichen Begleitung des Bund-Länder-Wettbewerbs Aufstieg durch Bildung: offene Hochschulen. Band 1, Münster. 



\title{
Wissenskooperation durch Tagungen und Publikationen
}

\author{
Wolfgang Jütte/Claudia Lobe/Markus Walber
}

\begin{abstract}
Der vorliegende Beitrag wirft einen Blick auf die „Kommunikationsinfrastruktur“ der DGWF: die Jahrestagungen und ihre Fachzeitschrift. Es wird nachgezeichnet, wie über die Jahrzehnte die Bedarfe an Kommunikation und ihre Formen einen kontinuierlichen Wandel erfahren haben. Dabei wird ein besonderer Blick auf unterschiedliche Akteursgruppen mit ihren jeweiligen Kommunikationsansprüchen geworfen. Ein Schwerpunkt wird auf die Auswertungsverfahren (Monitoring) der Jahrestagung und die veränderten Publikationsstrategien gelegt. ${ }^{1}$
\end{abstract}

\section{Zum Wissensaustausch in einer Fachgesellschaft}

In einer Fachgesellschaft kommt der wissenschaftlichen Kommunikation eine herausragende Bedeutung zu. Durch die Organisation von Tagungen und die Möglichkeiten des persönlichen Austausches prägt die DGWF als zentraler „Player“ seit Jahrzehnten das Feld. Zunächst war für den Arbeitskreis Universitäre Erwachsenenbildung (AUE) vor allem die Außenorientierung eine bedeutsame Aufgabe: So „erwies sich der AUE als ein wichtiges trägernahes Bindeglied, das zunehmend öffentliche Aufmerksamkeit fand, sich zugleich allerdings steigenden Erwartungen der Kooperationspartner gegenübersah“ (Dikau 1996, S. 29). In den folgenden Jahren profilierte und veränderte sich das institutionelle Selbstverständnis, sodass sie derzeit durchaus Funktionen einer Fachgesellschaft im klassischen Sinne übernommen hat. Deren Rollenvielfalt, so wie sie in einer aktuellen Untersuchung aus Großbritannien unterstrichen wird, trifft mittlerweile wohl auch umstandslos auf die DGWF zu:

„Learned societies are valued primarily for organizing conferences, publishing peer-reviewed academic journals, and defining and advocating for the field's values and policy priorities. Conferences are valued for their formal function of helping academics keep up with new scholarship, and the informal role of connecting academics with peers" (Housewright/Schonfeld/Wulfson 2013, S. 92).

1 Dieser Beitrag basiert u. a. auf vorherigen Ausführungen der Autoren (Jütte 2013, Jütte 2015, Lobe/Walber 2015, Lobe/ Walber 2016), ohne dass diese besonders gekennzeichnet sind. 
Im letzten Jahrzehnt ist eine generelle Zunahme von Tagungen und Konferenzen nicht zuletzt aufgrund der Zunahme von Projektförmigkeit - zu beobachten. Zugleich ist das allgemeine Publikationsaufkommen spürbar angestiegen.

In diesem Zuge hat in der DGWF auch eine Ausdifferenzierung nach innen stattgefunden. Mittlerweile existieren vier Arbeitsgemeinschaften und acht Landesgruppen (siehe dazu die Beiträge in diesem Band), die sich regelmäßig treffen und das Feld auf vielfältige Weise bearbeiten. Diese Vielfalt der Stimmen und Bedarfe schlägt sich auch in den Jahrestagungen nieder.

\section{Jahrestagungen als kommunikativer Fixpunkt}

Wissenschaftliche Konferenzen haben verschiedene Formen und Namen und sind je nach Fachkulturen recht unterschiedlich gestaltet. Fachgesellschaften mit einer Vereinsstruktur wie die DGWF nennen sie Jahrestagung. Damit wird die Periodizität ausgedrückt. Sie sind ein ,jährlicher Fixpunkt, bei dem die Experten wissenschaftlicher Weiterbildung unter einem aktuell gewählten Leitthema Daten und Erkenntnisse der Fachdisziplin zusammenführen und unter Kollegen kommunizieren" (Strittmatter-Haubold 2014, S. 81). Konferenzen leben vom mündlichen Austausch. Mit der zunehmenden Größe der Tagung entsteht ein besonderes Problem: Der Raum zum direkten Austausch wird knapp; dies schlägt sich auch in der Evaluation der Jahrestagungen nieder.

Die Konferenzen der DGWF werden seit ihrem frühen Beginn in den 1970er-Jahren - hier noch unter dem Namen AUE (Arbeitskreis universitäre Erwachsenenbildung) - jährlich durchgeführt. Im Folgenden sind die Tagungen von über vier Jahrzehnten (1970-2016) angeführt²:

- Die universitäre Erwachsenenbildung in der Technischen Welt (1970, Hannover)

- Weiterbildung als eine Aufgabe der Hochschulreform. Das Kooperationsfeld der universitären Erwachsenenbildung in Theorie und Praxis (1971, Frankfurt/ Main)

- Zieldimensionen und Organisationsstrukturen universitärer Erwachsenenbildung. Institutionalisierung wissenschaftlicher Weiterbildung (1972, Trier)

- Qualifikationsveränderungen und wissenschaftliche Weiterbildung (1973, München)

- Universitäre Erwachsenenbildung für wen und mit wem? (1974, Bochum)

- Projektplanung „Öffnung der Hochschulen durch den Ausbau der wissenschaftlichen Weiterbildung im Zusammenwirken von Hochschulen und Weiterbildungseinrichtungen des Einzugsbereichs“ (1975, Vallendar)

- Weiterbildung und Hochschule. Perspektive ohne Expansion? (1976, Oldenburg)

Wir danken Bernhard Christmann für seine Vorarbeiten. 
- Mitwirkung der Hochschulen in der Weiterbildung (1977, Augsburg)

- Hochschule und Weiterbildung. Bilanz und Perspektive nach 20 Jahren universitärer Erwachsenenbildung (1978, Göttingen)

- Das Arbeitsprogramm des AUE (1979, Bremen)

- Wissenschaftliche Weiterbildung im Spannungsfeld zwischen Hochschule und Öffentlichkeit (1980, Bamberg)

- Hochschule und Weiterbildung in Nordrhein-Westfalen (1981, Aachen)

- Weiterbildung in einem differenzierten Hochschulsystem (1982, Hamburg)

- Hochschule, Weiterbildung und Arbeitsmarkt (1983, Bad Zwischenahn)

- Wissenschaftliche Weiterbildung: Analysen, Reflexionen, Statements (1984, Regensburg)

- Hochschule in der Region. Wissenschaftliche Weiterbildung, Wissenstransfer, Serviceleistungen (1985, Osnabrück)

- Die Zukunft von Hochschule und Weiterbildung (1986, Soest)

- Weiterbildung an Hochschulen als öffentliche Aufgabe. Beiträge zur Infrastruktur des Bildungswesens (1987, Karlsruhe)

- Chancen und Barrieren der wissenschaftlichen Weiterbildung (1988, Bielefeld)

- Perspektiven der wissenschaftlichen Weiterbildung für die neunziger Jahre (1989, Hannover)

- Wissenschaftliche Weiterbildung als gemeinsame Aufgabe der deutschen Hochschulen (1990, Kiel)

- Europäische Integration als Herausforderung für Wissenschaft, Hochschule und Weiterbildung (1991, Konstanz)

- Wissenschaftliche Weiterbildung und Hochschulerneuerung (1992, Magdeburg)

- Fernstudium - ein wichtiger Impuls für die wissenschaftliche Weiterbildung (1993, Braunschweig)

- Wissenschaftliche Weiterbildung an Hochschulen. Ein innovatives Potential für die regionale Wirtschaft? (1994, Potsdam)

- Qualitätsmanagement in der wissenschaftlichen Weiterbildung (1995, Stuttgart)

- Perspektiven wissenschaftlicher Weiterbildung in Europa (1996, Regensburg)

- Wissenschaftliche Weiterbildung im Netz der Wissenschaften. Interdisziplinarität, Innovation, Transfer - Beispiele aus Umweltschutz und Gesundheit (1997, Flensburg)

- Weiterbildung als Kernaufgabe der Hochschulen. Wissenschaftliche Weiterbildung unter neuen Rahmenbedingungen (1998, Hamburg)

- Wissenschaftliche Weiterbildung in neuer Umgebung. Internationalisierung gestufte Abschlüsse - moderne Strukturen (1999, Bochum)

- Zukunftsforum wissenschaftliche Weiterbildung - Markt der wissenschaftlichen Weiterbildung (2000, Augsburg)

- Lernen ein Leben lang. Beiträge der wissenschaftlichen Weiterbildung: Hochschulpolitik - Strukturentwicklung - Qualitätssicherung - Praxisbeispiele (2001, Leipzig) 
-Weiterbildungsmanagement und Hochschulentwicklung. Die Zukunft gestalten (2002, Kaiserslautern)

- Profil und Qualität der wissenschaftlichen Weiterbildung zwischen Wirtschaftlichkeit und Wissenschaft (2003, Dresden)

- Wandel der Hochschulkulturen? Wissenschaftliche Weiterbildung zwischen Kultur und Kommerz (2004, Hannover)

- Wissenschaftliche Weiterbildung im Hochschulraum Europa (2005, Wien)

- Wissensmanagement und wissenschaftliche Weiterbildung (2006, Karlsruhe)

- Wa(h)re Bildung! Gegenwart und Zukunft der wissenschaftlichen Weiterbildung angesichts von Bologna und GATS (2007, Bern)

- Strukturwandel der Arbeit. Zukunft der wissenschaftlichen Weiterbildung (2008, Bochum)

- Wissenschaftliche Weiterbildung: Zehn Jahre nach Bologna. Alter Wein in neuen Schläuchen oder Paradigmenwechsel? (2009, Berlin)

- Hochschulen im Kontext lebenslangen Lernens. Konzepte, Modelle, Realität (2010, Regensburg)

- Produktive Grenzüberschreitungen in der wissenschaftlichen Weiterbildung. Öffnung - Interdisziplinarität - Transnationalisierung (2011, Bielefeld)

- Wächst zusammen, was zusammengehört? Wissenschaftliche Weiterbildung berufsbegleitendes Studium lebenslanges Lernen (2012, München)

- Auf dem Weg zur Hochschule des Lebenslangen Lernens. Mehrwert, Aufwand und Erträge (2013, Rostock)

- Wissenschaftliche Weiterbildung neu denken! Ansätze und Modelle für eine innovative Gestaltung von Weiterbildung und lebenslangem Lernen an Hochschulen (2014, Hamburg)

- Lehr-/Lernarrangements in der wissenschaftlichen Weiterbildung - Herausforderungen und Erfolgsfaktoren für eine wirksame Didaktik (2015, Freiburg)

- Die Vielfalt der Lifelong Learners - Herausforderungen für die Weiterbildung an Hochschulen (2016, Wien)

Ein erster Blick zeigt: Seit ihren Ursprüngen sind die Themen aktuell und „modern“. Eine differenziertere Analyse, die nicht allein auf die Konjunktur der Themen abzielt, sondern auch die Größe der Veranstaltung, die Zielgruppen, den Ablauf und die Organisation etc. mit in den Blick nimmt, wäre ein lohnenswertes Unterfangen.

Ernst Prokop (1996) hat in einer Zwischenaufnahme den Stellenwert der Tagungen zu Beginn des AUE beleuchtet: „Die Jahrestagungen hatten eine repräsentative Funktion gegenüber der Öffentlichkeit. Sie dienten von vornherein dazu, den innovativen Akzent unübersehbar zu präsentieren, der darin gesetzt wurde, die Weiterbildung als dritte Hochschulaufgabe neben Forschung und Lehre zu betonen. So verbanden sich die Themen von Jahrestagungen im Jahrzehnt der Gründung häufig mit den, dem Zeitgeist verbundenen Erörterungen“ (ebd., S. 129). Dieser Grundgedanke kann sicherlich bis heute beobachtet werden. Womöglich ist der Stellenwert der Jahrestagungen als Ort für den wissenschaftlichen Austausch gestiegen. 
Die Vorträge der Jahrestagung wurden in der Regel in einem Tagungsband in Buchform von einem Herausgeber-Komitee redigiert und Monate später in den „Beiträgen“ veröffentlicht. Mit der Neuausrichtung der „Hochschule und Weiterbildung“ (s. Kap.4) und den erweiterten medialen Möglichkeiten erfuhr dieses Vorgehen eine Veränderung und die Reihe „Beiträge“ wurde eingestellt. Für die Dokumentation der Jahrestagung in Hamburg (2014) wurde erstmals ein Abstract-Band online veröffentlicht. Seitdem werden die Konferenzbeiträge regelmäßig in Form von Abstract-Bänden zeitnah in elektronischer Form auf der Website der DGWF einer breiten Fachöffentlichkeit zur Verfügung gestellt. Dies ermöglicht es, sich schnell einen Überblick über vergangene Jahrestagungen zu verschaffen sowie Themen und Projekte zu identifizieren. Ein weiterer Vorteil liegt darin, dass die Beiträge den „inneren Zirkel“ der Fachgesellschaft überschreiten und sichtbarer werden. Hier zeichnet sich ab, dass die Digitalisierung auch einen Wandel in der Kommunikation beschleunigt. Insofern ist sicherlich nicht zufällig, dass die Website der DGWF (www. dgwf.net) an Stellenwert für die Kommunikation nach außen und innen an Bedeutung gewinnt.

\section{Die Vielfalt der Akteure. Von der Tagungsevaluation zum Monitoring}

Eine Evaluation der Jahrestagungen wurde erstmals 2007 eingeführt und zunächst von der Universität Hamburg realisiert. Seit 2014 wird die Erhebung an der Universität Bielefeld von einer Forschergruppe am Institut für wissenschaftliche Weiterbildung (IWW) vorgenommen (Lobe/Walber/Wistinghausen 2015, Lobe/Walber 2016, 2017).

Mit dem Wechsel entstand die Idee, die Tagungsevaluation nicht ausschließlich als Qualitätssicherungsmaßnahme für die inhaltliche, didaktische und organisatorische Verbesserung der Tagung einzusetzen. Fraglos ist dies eine wichtige Funktion der Tagungsevaluation, die auch in Zukunft fortbestehen soll. Darüber hinaus liegt es aber nahe, die Tagungsevaluationen auch als Berichtssystem zu verstehen, das in jährlicher Periodizität Auskunft über aktuelle Trends, thematische Präferenzen und die Zusammensetzung der Akteure in der wissenschaftlichen Weiterbildung geben kann. Im Zeitverlauf werden so Veränderungen und Verschiebungen sichtbar, die sich - wie dies z. B. in der Trendforschung oder im Bildungsmonitoring geschieht kontinuierlich erfassen und auswerten lassen. Die hier angestrebte Auswertung erhebt dabei nicht den Anspruch, zukünftige Entwicklungen zu prognostizieren oder bildungspolitische Steuerung zu unterstützen, sondern versteht sich als periodisches Beobachtungsinstrument, das Entwicklungen in der wissenschaftlichen Weiterbildung aus der Perspektive ihrer Fachgesellschaft heraus nachhält und dokumentiert. Ihr Potenzial wird sich vor allem zukünftig anhand von Zeitreihenanalysen zeigen.

Für die DGWF-Jahrestagung 2014 in Hamburg wurde insofern der bisherige Evaluationsfragebogen einer kritischen Revision unterzogen und für einen kontinuierli- 
chen Einsatz adaptiert. Dabei wird insbesondere folgenden Fragestellungen nachgegangen: Wie setzen sich die Teilnehmenden im Hinblick auf ihre berufliche Beschäftigung mit wissenschaftlicher Weiterbildung zusammen? Welchen Institutionen gehören sie an, welche Tätigkeiten üben sie schwerpunktmäßig aus, was motiviert sie zur Teilnahme und für welche Themen interessieren sie sich besonders? Von Interesse ist zudem: Wie strukturiert sich das „Einzugsgebiet“ der DGWF in Deutschland und darüber hinaus, wie ist das Verhältnis zwischen Mitgliedern und Nicht-Mitgliedern auf den Jahrestagungen, wie ist das Verhältnis zwischen Mitgestaltenden (Referent_innen, Moderator_innen) und "passiv“ Teilnehmenden? Und schließlich stellt sich die Frage: Unterscheiden sich verschiedene Teilnehmergruppen systematisch in ihren thematischen Präferenzen oder der Bewertung des Tagungsoutcomes?

Inzwischen liegen die Evaluationsergebnisse der DGWF-Jahrestagungen aus den Jahren 2014 bis 2016 vor, die sich zueinander sowie vereinzelt auch zu Daten aus dem Jahr 2013 in Bezug setzen lassen. Im Folgenden werden ausgewählte Erkenntnisse vorgestellt mit dem Blick auf die Zielgruppen der Jahrestagungen. ${ }^{3}$ Herausgegriffen werden hier folgende drei Gesichtspunkte:

- Veränderungen in der Teilnehmerstruktur der Jahrestagungen

- Veränderungen in den Teilnahmemotiven und -profilen und die Analyse von Zusammenhängen mit berufsbezogenen Merkmalen

- Veränderungen in den Bewertungen der Jahrestagungen und die Analyse von Zusammenhängen mit berufsbezogenen Merkmalen.

\subsection{Teilnehmerstruktur}

Die Altersstruktur zeigt, dass sich der Altersdurchschnitt in den letzten Jahren kontinuierlich leicht verringert hat (s. Abb. 1). Dies zeigt sich sowohl an den Mittelwerten als auch am Median, der mittlerweile bei 38 Jahren liegt. Auffällig ist, dass die „sehr Alten“ (70+) 2016 gar nicht mehr vertreten waren und zugleich bereits „sehr Junge“ (18) Teilnehmer_innen zu verzeichnen sind.

Dies könnte dafür sprechen, dass die Jahrestagungen für Studierende und Nachwuchswissenschaftler_innen zunehmend interessant werden, das Interesse der „Ruheständler_innen“ dagegen eher abnimmt.

Wir danken Marielle Thünemann für ihre Unterstützung im Auswertungsprozess. 


\begin{tabular}{|l|c|c|c|}
\hline Statistische Maße & $\mathbf{2 0 1 4}$ & $\mathbf{2 0 1 5}$ & $\mathbf{2 0 1 6}$ \\
\hline Gültig & 158 & 164 & 186 \\
\hline Fehlend & 13 & 19 & 9 \\
\hline Mittelwert & 42,77 & 42,41 & 40,67 \\
\hline Median & 42 & 40 & 38 \\
\hline Standardabweichung & 11,74 & 11,27 & 18,43 \\
\hline Minimum & 25 & 26 & 69 \\
\hline Maximum & 85 & 86 & 18 \\
\hline
\end{tabular}

Abb. 1: Altersverteilung der Stichprobe im Zeitverlauf (2014-2016)

Die Mehrheit der Teilnehmenden ist zu etwa zwei Dritteln weiblich. Im Zeitverlauf zeigt sich, dass der Anteil der Teilnehmerinnen kontinuierlich leicht angestiegen ist (s. Abb. 2).

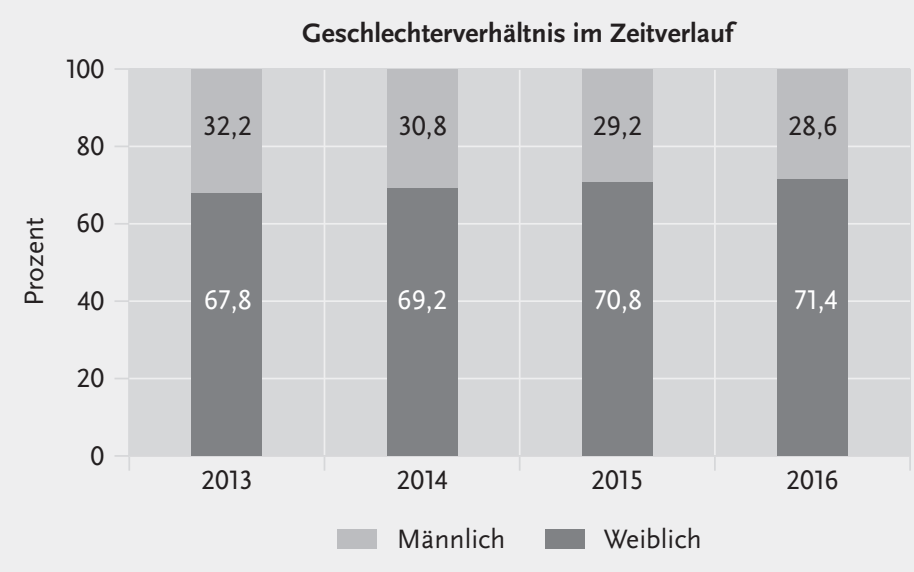

Abb. 2: Geschlechterverhältnis der Stichprobe im Zeitverlauf (2013-2016) in Prozent

Die Tendenzen bezüglich Alter und Geschlecht könnten mit dem Programm „Aufstieg durch Bildung - Offene Hochschulen“ zusammenhängen, durch das im Feld der wissenschaftlichen Weiterbildung zahlreiche Projektstellen für den wissenschaftlichen Nachwuchs geschaffen wurden. Die in diesem Jahr neu eingeführte Frage nach der Zugehörigkeit der Teilnehmenden zu einem Projekt dieses Programms ergibt, dass knapp ein Drittel (30,5\%) der Befragten in einem „Offene-HochschuleProjekt“ angestellt ist (s. Abb. 3). 


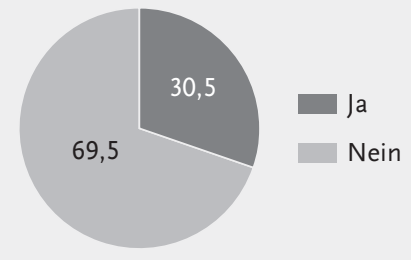

Abb. 3: Mitgliedschaft im Programm „Offene Hochschule“ 2016 in Prozent

Die institutionellen Kontexte, in denen die Teilnehmenden mit wissenschaftlicher Weiterbildung befasst sind, erweisen sich wie in den vergangenen Erhebungsjahren als fast ausschließlich hochschulisch geprägt (s. Abb.4).

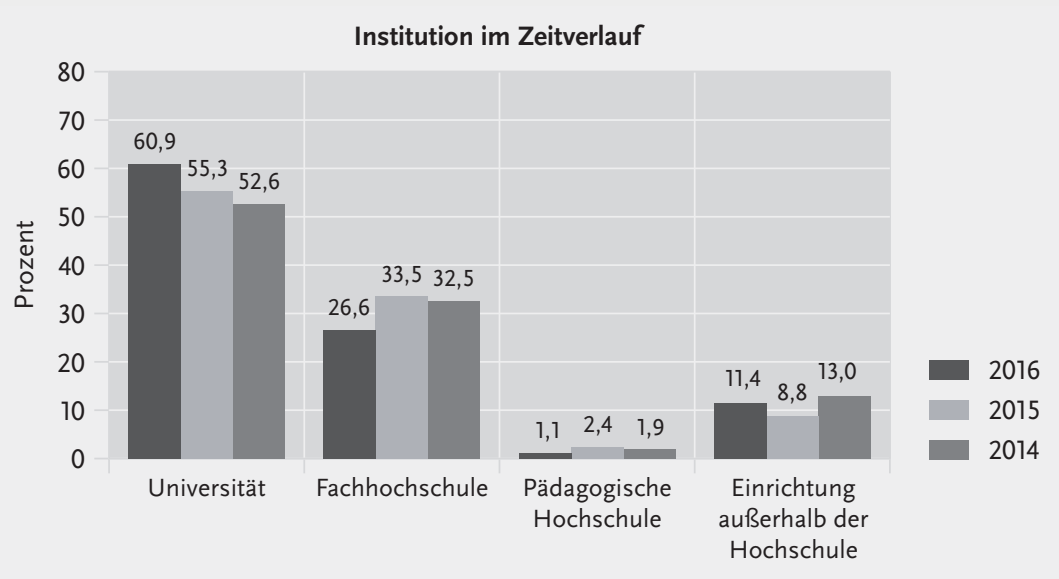

Abb. 4: Institution im Zeitverlauf (2014-2016) in Prozent

Der weit überwiegende und in den letzten Jahren gestiegene Anteil der Teilnehmenden ist an Universitäten tätig, während nur etwa ein knappes Drittel der Befragten an Fachhochschulen beschäftigt ist (2016 sind es sogar nur gut ein Viertel). Teilnehmende aus Pädagogischen Hochschulen bilden jeweils nur einen marginalen Anteil der Befragten. Um die zehn Prozent der Teilnehmenden ordnen sich jeweils Einrichtungen außerhalb der Hochschule zu. Die institutionellen Teilnahmeprofile erweisen sich insgesamt im Zeitverlauf als relativ stabil.

Die im Jahr 2015 neu eingeführte Frage nach der Trägerschaft der Institutionen zeigt auch im Jahr 2016, dass Teilnehmende aus öffentlichen Einrichtungen gegenüber Teilnehmenden aus privaten Einrichtungen in der Stichprobe jeweils deutlich überwiegen: Etwa zwei Drittel der Einrichtungen außerhalb der Hochschule (65,0\%) und der weit überwiegende Teil der Fachhochschulen (95,9\%) und Universitäten $(98,2 \%)$ stehen in öffentlicher Trägerschaft. 
Neben dem institutionellen Kontext wurden auch die Tätigkeiten erfragt, mit denen die Teilnehmenden im Bereich der wissenschaftlichen Weiterbildung befasst sind. Die Befragten wurden gebeten, den Umfang der einzelnen Tätigkeitsbereiche auf einer Skala von 1 (gar nicht) bis 6 (sehr) einzuschätzen. Die folgende Abbildung (s. Abb. 5) gibt Aufschluss über den Umfang der Tätigkeiten in den Bereichen Administration/Operatives Management, Wissenschaft/Forschung, Beratung, Leitung/Strategisches Management, Lehre sowie Politik. Im Folgenden wird für die sechs Tätigkeitsfelder jeweils der Mittelwert angegeben.

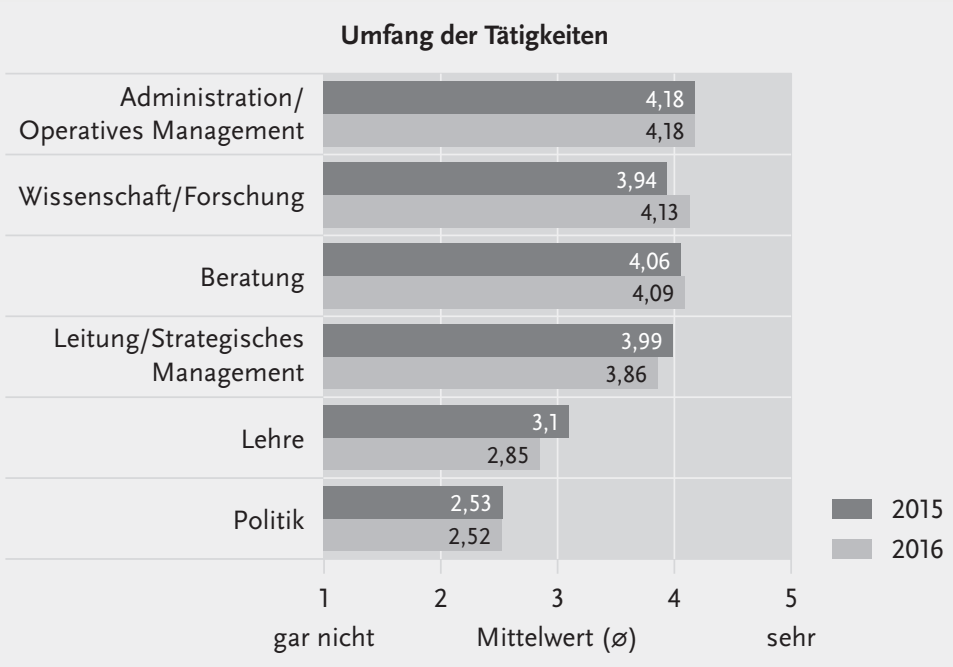

Abb. 5: Mittelwerte der einzelnen Tätigkeitsbereiche im Zeitverlauf (2015-2016)

Im Jahr 2016 sind die Befragten insbesondere in den Tätigkeitsfeldern Administration/Operatives Management und Wissenschaft/Forschung aktiv, gefolgt von den Tätigkeitsfeldern Beratung und Leitung/Strategisches Management. Die Tätigkeitsfelder Lehre und Politik spielen dagegen im Durchschnitt eher eine untergeordnete Rolle in den Tätigkeitsprofilen der Teilnehmenden. ${ }^{4}$

Zusätzlich zu den personenbezogenen Sozialdaten und den berufsbezogenen Informationen wurde der Rolle genauer nachgegangen, die die Teilnehmenden während der Tagung innehatten. Zudem wurde geprüft, in welchem Verhältnis die Teilnehmenden zur DGWF stehen. Hinsichtlich der Tagungsrolle zeigt sich, dass 40,6 Prozent der Befragten auf der Tagung vortrugen oder als Moderator_innen fungierten.

4 Die Standardabweichungen für die einzelnen Tätigkeitsbereiche liegen zwischen 1,6 und 2,1, sodass sie etwa anderthalb bis zwei Skalenstufen auf der sechsstufigen Bewertungsskala umfassen. Die relativ hohen Mittelwerte sprechen dafür, dass die meisten Befragten tatsächlich in mehreren Tätigkeitsfeldern aktiv sind, wobei das Ausmaß der Standardabweichungen auf eine starke Streuung der Werte und somit auf recht unterschiedliche Gewichtungen der Tätigkeitsfelder in den Tätigkeitsprofilen der Einzelnen hindeutet. 
Die meisten (59,4\%) der Teilnehmenden aus der Stichprobe hatten keine dieser Rollen inne und waren somit ausschließlich Teilnehmer_innen (s. Abb. 6).

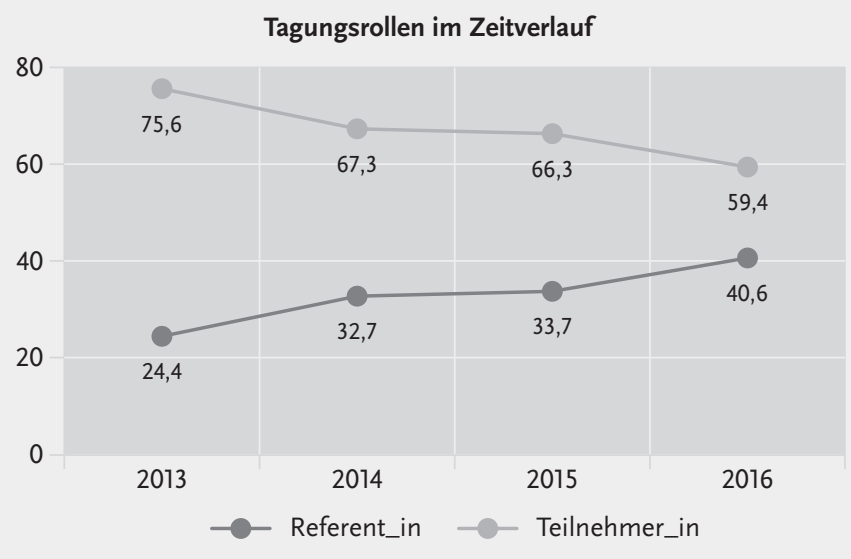

Abb. 6: Tagungsrollen im Zeitverlauf (2013-2016) in Prozent

Im Zeitverlauf wird deutlich, dass die Zahl der Referent_innen in den Jahren 2013 bis 2016 insgesamt um 16,2 Prozent angestiegen ist. Dies spricht für ein hohes Interesse an der Mitgestaltung der Tagung, was für eine Expansion der Vortrags-Slots im Tagungsprogramm sorgt.

Bezüglich der Mitgliedschaft in der $\mathrm{DGWF}^{5}$ wird deutlich, dass über die Hälfte der Befragten (56,8\%) Mitglieder der DGWF sind (s. Abb.7). Etwa jede_r zehnte Befragte $(10,9 \%)$ ist zugleich Funktionsträger_in (Vorstand, Beirat, Programmkommission).

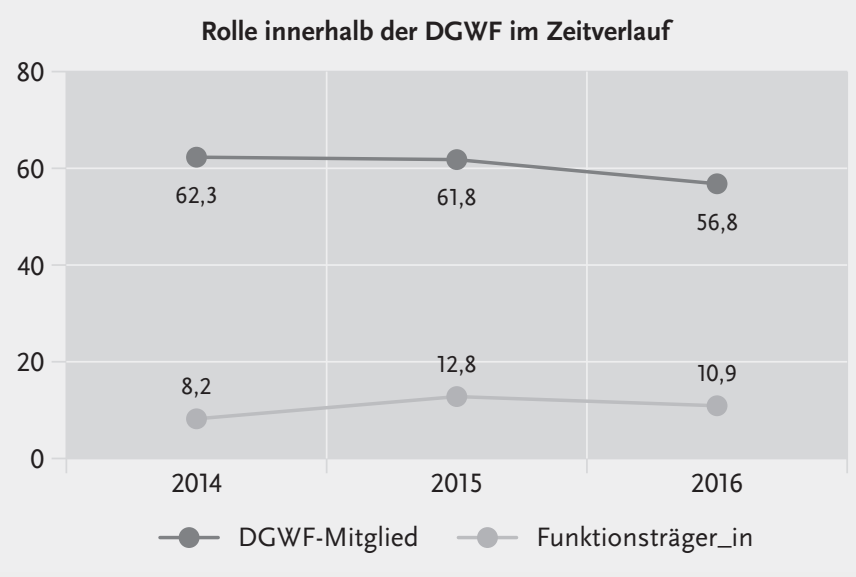

Abb. 7: Rolle innerhalb der DGWF im Zeitverlauf (2014-2016) in Prozent

5 Im Jahr 2014 wurde nach einer Mitgliedschaft in einer „Vereinigung der wissenschaftlichen Weiterbildung“ gefragt; seit 2015 explizit nach einer „Mitgliedschaft in der DGWF“. 
Damit hat sich der Anteil der DGWF-Mitglieder in der Stichprobe seit dem Jahr 2014 um 5,5 Prozent verringert und es nehmen verstärkt auch Nicht-Mitglieder an der Tagung teil.

\subsection{Teilnahmemotivationen und -profile}

Um Aufschluss über die Teilnahmemotivation der Befragten zu erhalten, wurden acht Motivationsdimensionen unterschieden und auf einer sechsstufigen Skala erfasst: Inhaltlicher Austausch, Sozialer Austausch, Vernetzung und Kooperation, Attraktivität des Tagungsorts, Aktualität des Themas, Praktische Themen, Wissenschaftliche Themen und Empirische Ergebnisse. Die Teilnehmer_innen wurden gebeten, die Bedeutung der einzelnen Motivationsdimensionen für ihre Teilnahme an der Jahrestagung auf einer Skala von 1 (unwichtig) bis 6 (sehr wichtig) zu bewerten. Folgende Abbildung (s. Abb. 8) zeigt die Mittelwerte für die Motivationsdimensionen im Zeitverlauf.

Wie in den beiden Vorjahren zeigt sich auch im Jahr 2016, dass alle Motivationsdimensionen von den Befragten als relativ wichtig für die Teilnahmeentscheidung eingeschätzt werden, sodass alle Mittelwerte im Bereich der oberen Skalenhälfte ange-

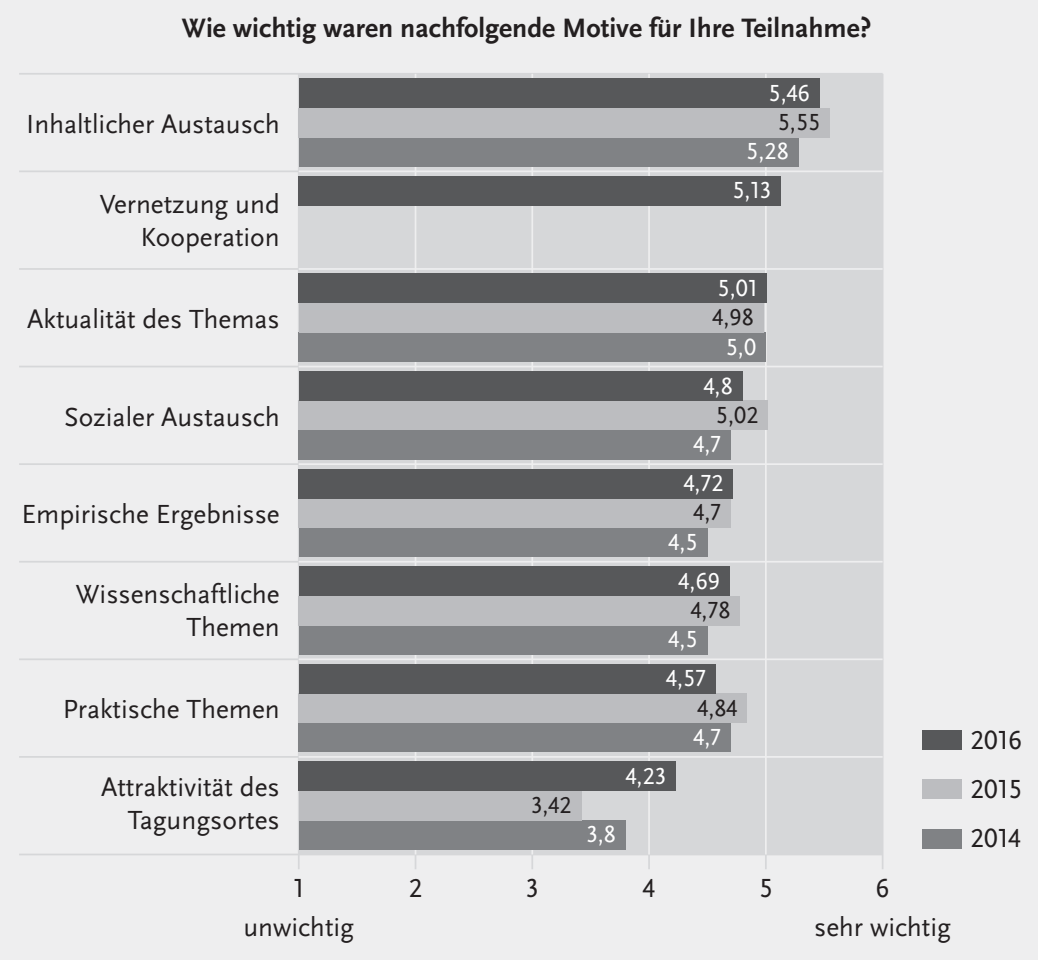

Abb. 8: Mittelwertvergleich der Motivationsitems im Zeitverlauf (2014-2016) 
siedelt sind. Aus dem Vergleich der Evaluationsergebnisse der Jahre 2014 bis 2016 gehen nur leichte Unterschiede im Hinblick auf die Motive der Tagungsteilnahme hervor. In allen drei Erhebungsjahren wurde dem inhaltlichen Austausch die höchste Priorität beigemessen, während der Attraktivität des Tagungsorts im Vergleich jeweils eher eine untergeordnete Rolle zukommt. Im Vergleich zum Vorjahr 2015 (Freiburg) verzeichnet diese Dimension jedoch für den Tagungsort 2016 in Wien einen Zuwachs von o,81. Auffällig ist, dass die 2016 neu aufgenommene Motivationsdimension „Vernetzung und Kooperation“ von den Teilnehmenden mit einem Mittelwert von 5,13 stark frequentiert wurde.

Es wurde nun geprüft, inwiefern die Motivationsdimensionen mit unterschiedlichen Tätigkeitsschwerpunkten im Feld der wissenschaftlichen Weiterbildung zusammenhängen (s. Abb. 9).

Die Grafik zeigt, dass unterschiedliche Tätigkeitsfelder mit je spezifischen Teilnahmemotivationen einhergehen. Während ein hohes Engagement im Bereich der Wissenschaft/Forschung mit einem hohen Interesse an wissenschaftlichen Themen und empirischen Ergebnissen einhergeht, stehen Leitungsaufgaben vor allem mit einem Interesse an Vernetzung und Kooperation in Zusammenhang. Sowohl praktische Themen als auch die Aktualität des Tagungsthemas bekommen vor allem von den Befragten, die sich stark mit Beratung befassen, eine hohe Wichtigkeit zugesprochen. Praktische Themen sind in besonderem Maße auch für die befragten Teilnehmer_innen aus dem Bereich der Administration bzw. des operativen Managements von hoher Relevanz, während hier hingegen ein negativer Zusammenhang zwischen empirischen Ergebnissen und wissenschaftlichen Themen erkennbar ist. Für das Tätigkeitsfeld der Politik zeigen sich positive Zusammenhänge im Hinblick auf Vernetzung und Kooperation sowie praktische Themen, während hinsichtlich empirischer Ergebnisse ein negativer Zusammenhang besteht. Ein Vergleich mit den Korrelationskoeffizienten aus dem Jahr 2015 ergibt, dass sich die Tendenzen der meisten Tätigkeitsgruppen als relativ stabil erweisen. Hierin liegt also ein großes Potenzial für die genauere Analyse der Zielgruppen und Interessenlagen der Tagungsteilnehmer_innen. 


\section{Zusammenhang zwischen Tätigkeit und Teilnahmemotivation}

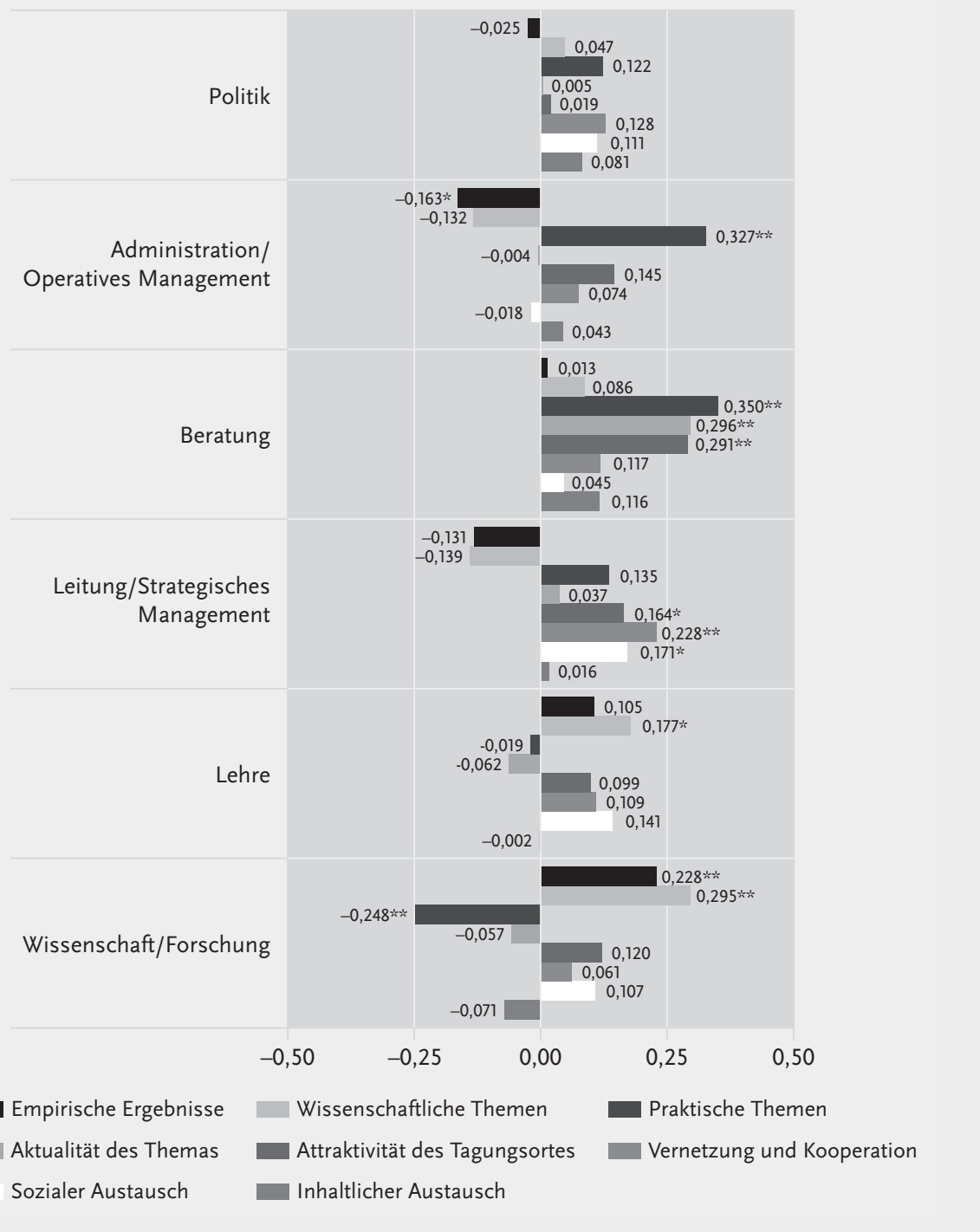

Abb. 9: Zusammenhangsstärke (Pearson $\left.{ }^{6}\right)$ zwischen Tätigkeit und Teilnahmemotivation 2016

6 Der Korrelationskoeffizient gibt an, wie stark die Zusammenhänge zwischen der Gesamtzufriedenheit und den jeweiligen Teilnahmemotiven ausfallen. 


\subsection{Teilnehmerbewertungen}

Es wurde auch der Frage nachgegangen, inwiefern die Variable „Gesamtzufriedenheit“ mit den Teilnahmemotiven in Zusammenhang steht (s. Abb.10) ${ }^{7}$. Dies gibt Auskunft darüber, inwiefern die Tagung den Erwartungshorizonten der Teilnehmenden entsprochen hat.

Die Daten für das Jahr 2016 zeigen positive Korrelationen für alle Motivationsdimensionen mit Ausnahme der Dimension „Praktische Themen“. Hier ergibt sich eine negative Korrelation. Dies lässt sich so deuten, dass den Erwartungen der Befragten durch die Tagungsgestaltung tendenziell entsprochen wurde, die Erwartungen der Teilnehmenden in der Motivationsdimension „Praktische Themen“ hingegen tendenziell nicht erfüllt wurden. Die Stärke der Zusammenhänge mit der Gesamtzufriedenheit variiert allerdings für verschiedene Motivationsdimensionen. ${ }^{8}$ Insgesamt zeigt sich eine hohe Pluralität im Zeitverlauf, was dafür spricht, dass jede Jahres-

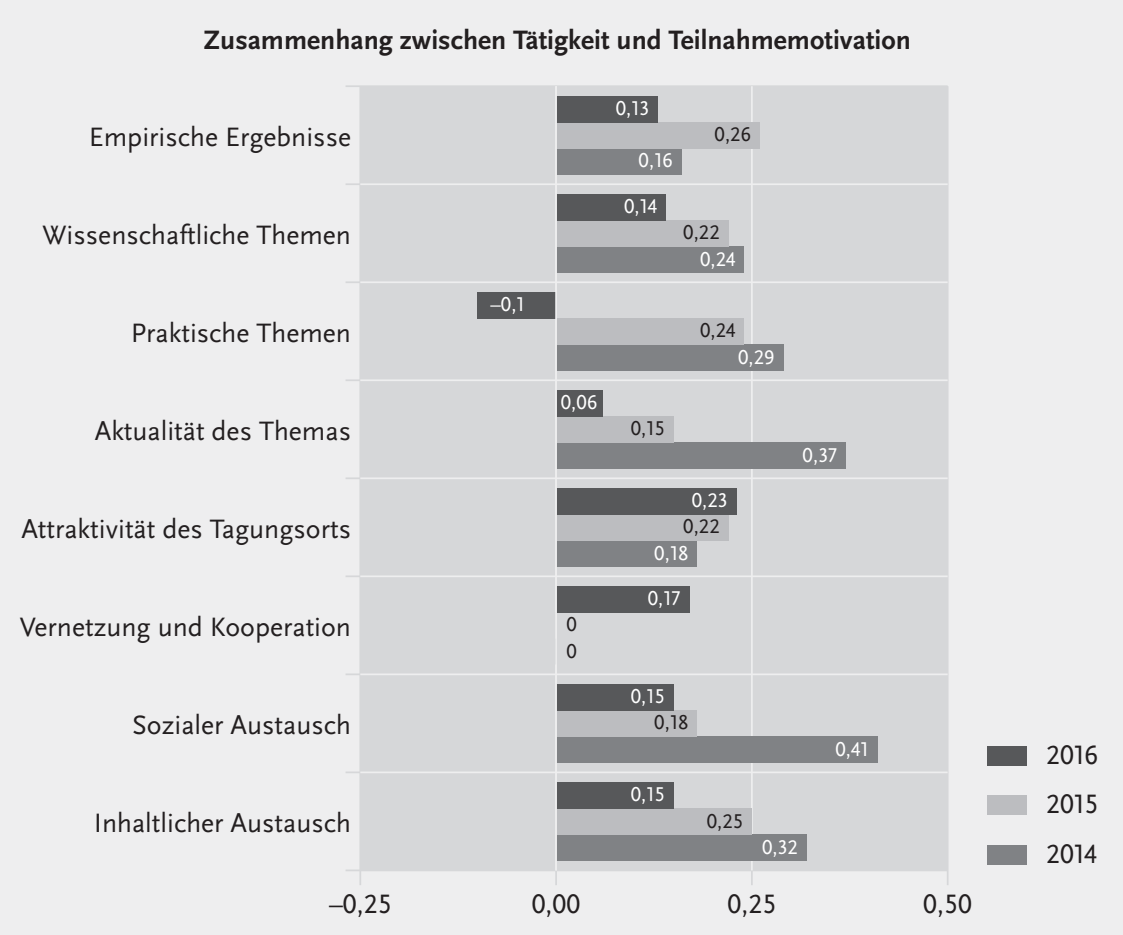

Abb. 10: Zusammenhangsstärke (Pearson) zwischen Gesamtzufriedenheit und Teilnahmemotivation 2016

7 In den Jahren 2014 und 2015 wurde jeweils aus allen bewertungsbezogenen Variablen ein Index zur Gesamtzufriedenheit errechnet. Seit 2016 wird die Gesamtzufriedenheit in einem eigenen Item explizit erfragt.

8 Die Korrelationen fallen im Jahr 2016 tendenziell schwächer aus, was an den methodischen Änderungen von einem errechneten Zufriedenheits-Index zu einem eigenen Item zur Erfassung der Gesamtzufriedenheit liegen kann und in den kommenden Befragungen zu beobachten sein wird. 
tagung im Hinblick auf die Analysedimensionen (empirische Ergebnisse, praktische Themen etc.) eigene Schwerpunkte setzt. Die stärksten Zusammenhänge lassen sich für 2016 zwischen der Gesamtzufriedenheit und den Teilnahmemotiven „Attraktivität des Tagungsortes“ und „Vernetzung und Kooperation“ feststellen. Die Fortsetzung der Zeitreihen in den kommenden Jahren wird Aufschluss darüber geben, ob sich spezifische Tagungsprofile etablieren. Darüber hinaus lassen sie sich als Planungsinstrument für eine gezielte Ausrichtung der Tagungen nutzen.

\section{Vom „AUE-Info“ zur Zeitschrift für Hochschule und Weiterbildung (ZHWB)}

Die Zeitschrift war schon immer ein wichtiger Ort der Information. Zunächst erschienen in unregelmäßiger, aber mit 300 Ausgaben in sehr häufiger Folge bis 1982 die „AUE Informationen“. Sie waren geprägt vom internen Kommunikationsbedarf. Heute in Zeiten des Internets ersetzen „Rundmails“ diese Form der Kommunikation. Der seit 1983 regelmäßig erscheinende „Informationsdienst Hochschule und Weiterbildung“ entwickelte sich als „Stimme des AUE in der bildungspolitischen Öffentlichkeit“ (Prokop 1996, S.135). Im letzten Jahrzehnt, spätestens seit der Umbenennung des Arbeitskreises Universitäre Erwachsenenbildung (AUE) in „Deutsche Gesellschaft für wissenschaftliche Weiterbildung und Fernstudium“ (DGWF) im Jahre 2003, wies dieses Publikationsorgan immer mehr Charakteristika einer wissenschaftlichen Fachzeitschrift auf. „Hochschule und Weiterbildung“ war seit ihrem Beginn - neben den Jahrestagungen und Arbeitstreffen - ein bedeutendes Medium, um sich über aktuelle Trends im Bereich der wissenschaftlichen Weiterbildung zu informieren.

Mit der Übernahme der Funktion des geschäftsführenden Herausgebers durch Wolfgang Jütte und einem sukzessiv erweiterten Redaktionsteam (Claudia Lobe, Maria Kondratjuk, Mandy Schulze, Therese Zimmermann) erfuhr seit 2013 die kommunikationsstrategische Ausrichtung der Zeitschrift „Hochschule und Weiterbildung“ kontinuierliche Veränderungen. Das Profil wurde geschärft und die Ansprüche an die Qualität schrittweise erhöht (Jütte 2013). Seit 2014 werden die Zeitschriften auf www.pedocs.de als „delayed access“ veröffentlicht; die Downloadzahlen betragen derzeit monatlich ca. 400 Artikel.

$\mathrm{Zu}$ folgenden Themen erschienen seit 2013 die Schwerpunkthefte:

- Erfolgskonzept Fernstudium: Betreuung, Individualisierung, Methodenmix und Virtualität (2013/1)

- Reorganisation wissenschaftlicher Weiterbildung (2013/2)

- Auf dem Weg zur Hochschule Lebenslangen Lernens: Mehrwert, Aufwand und Erträge (2014/1)

- Wissenschaftliche Weiterbildung und Hochschuldidaktik (2014/2)

- Innovative Gestaltung von Weiterbildung und lebenslangem Lernen an Hochschulen (2015/1) 
-Wissenschaftliche Weiterbildung und gesellschaftliche Verantwortung $(2015 / 2)$

- Hochschulweiterbildung und Beruf (2016/1)

- Forschung auf und in Wissenschaftliche(r) Weiterbildung (2016/2)

In den nächsten Jahren wird eine weitere qualitative Veränderung angestrebt. Ein bedeutsamer Schritt ist die Entscheidung, die Zeitschrift ab 2017 nicht nur als PrintPublikation, sondern zusätzlich auch als E-Journal erscheinen zu lassen. Mit diesem Schritt leistet die DGWF einen sichtbaren Beitrag zur wissenschaftspolitisch bedeutsamen Open Access-Strategie. Zugleich sollen die Ausgaben und Beiträge übersichtlich zum Recherchieren und zum Download unter der Domain www.hochschuleund-weiterbildung.net zur Verfügung stehen, die im Besitz der DGWF ist. Ebenfalls erfolgt damit die Einführung eines Peer-Review-Systems für die Themenbeiträge; dies ist auch dem Umstand geschuldet, dass Nachwuchswissenschaftler_innen Veröffentlichungen in „peer reviewten“ Fachzeitschriften suchen. Die Präzisierung des Namens in "Zeitschrift für Hochschule und Weiterbildung“ (zhwb) ist daher nur konsequent.

Die Publikationsstrategie reagiert nicht zuletzt auf die Tatsache, dass Zeitschriften zunehmend eine dominante Funktion im Wissenschaftsbetrieb einnehmen. Eine Herausforderung wird darin bestehen, den unterschiedlichen Interessen der DGWFMitglieder, so wie sie sich auch in der Teilnahmemotivation bei Jahrestagungen niederschlagen, ebenfalls in der Ausrichtung der Zeitschrift Rechnung zu tragen; wenngleich wir über das konkrete Rezeptions- und Publikationsverhalten im Feld wissenschaftlicher Weiterbildung noch wenig wissen.

\section{Literatur}

Dikau, J. (1996): Die Entwicklung des AUE bis zur Wiedervereinigung Deutschlands. In: Dikau, J./Nerlich, B./Schäfer, E. (Hrsg.): Der AUE an der Schnittstelle zwischen tertiärem und quartärem Bildungsbereich - Bilanz und Perspektive. Bielefeld, 24-39.

Housewright, R./Schonfeld, R. C./Wulfson, K. (2013): Ithaka S+R/Jisc /RLUK UK Survey of Academics 2012. London: University of London.

Jütte, W. (2013): Stichwort: Verändertes Publikations- und Rezeptionsverhalten im Feld wissenschaftlicher Weiterbildung. In: Hochschule und Weiterbildung, 9-13.

Jütte, W. (2014): Stichwort: Konferenzen. In: Hochschule und Weiterbildung, (1), 7-9.

Lobe, C./Walber, M./Wistinghausen, M. (2015): Evaluation zur DGWF-Jahrestagung 2014 an der Universität Hamburg. Auftakt für ein Berichtssystem in der wissenschaftlichen Weiterbildung. Bielefeld: DGWF. Online: https://dgwf.net/fileadmin/user_upload/Jah restagung/2014/DGWF-Jahrestagung_2014_Evaluationsbericht.pdf (Stand: 08.11.2016). 
Lobe, C./Walber, M. (2016): Evaluation zur DGWF-Jahrestagung 2015 an der Universität Freiburg. Erste Zeitreihendaten im Berichtssystem zur wissenschaftlichen Weiterbildung. Bielefeld: DGWF. Online: https://dgwf.net/fileadmin/user_upload/Jahresta gung/2015/Evaluationsbericht_DGWF-Jahrestagung_2015.pdf (Stand: 28.10.2016).

Lobe, C./Walber, M. (2017): Evaluation zur DGWF-Jahrestagung 2016 an der Universität Wien. Berichtssystem zur wissenschaftlichen Weiterbildung. Bielefeld: DGWF (im Erscheinen).

Prokop, E. (1996): Tagungen und Publikationen des AUE zu Hochschule und Weiterbildung. In: Dikau, J./Nerlich, B./ Schäfer, E. (Hrsg.): Der AUE an der Schnittstelle zwischen tertiärem und quartärem Bildungsbereich. Bilanz und Perspektive, Bielefeld, $128-136$.

Strittmatter-Haubold, V. (2014): Die DGWF erprobt dialogorientiertes Tagungsformat Jahrestagung der DGWF 25.-27. September 2013 an der Universität Rostock. In: Hochschule und Weiterbildung, (2), 81-86. 



\section{Thematische Bereiche: die Arbeitsgemeinschaften}





\section{Die Arbeitsgemeinschaft der Einrichtungen für Weiterbildung an Hochschulen (AG-E)}

Karla Kamps-Haller/Bernhard Christmann/Helmut Vogt

Im Folgenden wird ein Gespräch zwischen Karla Kamps-Haller, Bernhard Christmann (BC) und Helmut Vogt (HV) wiedergegeben; die kursiven Passagen sind Fragen und Anmerkungen von Karla Kamps-Haller.

Die AG-E ist die älteste Sektion der DGWF. Zwei Personen sind Männer der ersten Stunde: Bernhard Christmann von der Ruhr-Universität Bochum leitete und prägte die AG-E über viele Jahre. Helmut Vogt von der Universität Hamburg hat die AG-E seit ihrer Gründung u. a. auch als geschäftsführender Vorstand der DGWF begleitet.

Deshalb wird dieser Beitrag in Form eines Gespräches mit diesen beiden Kollegen geführt, die inzwischen aus dem aktiven Berufsleben an ihren Einrichtungen ausgeschieden sind, aber nach wie vor Akteure der wissenschaftlichen Weiterbildung bleiben.

\section{Wie alles anfing und wie sich die AG-E weiterentwickelte}

Bernhard, ich glaube du warst ein Mann der ersten Stunde bereits vor der Gründung der AG-E. Seit wann warst du dabei und in welcher Rolle?

BC: Was die AG-E betrifft, war ich in der Tat von Anfang an dabei, als Mitglied der Projektgruppe des sogenannten Kontaktstellenprojektes des AUE. Ich war dort Vertreter der Ruhr-Universität Bochum bzw. unseres Modellversuchs KONRUB. Das Kontaktstellenprojekt war ein Projekt zur Erhebung aller damals existierenden Einrichtungen für Weiterbildung an Hochschulen, das der AUE durchgeführt hat. Hierzu gab es 1981 eine vorbereitende Tagung an der Universität Kassel, zu der alle Einrichtungen - es waren damals 21 - eingeladen waren. Ursprünglich war von Projekt- bzw. Vorstandsseite des AUE geplant, dass es einen kleinen Beirat geben sollte, in dem sechs bis acht Einrichtungen nach regionalem Proporz vertreten sein sollten. 
Es gab aber bereits bei der vorbereitenden Tagung aus dem Kreis der Einrichtungen ein sehr starkes Interesse, dass alle Vertreter der Einrichtungen an dem gesamten Prozess beteiligt sein sollten. Das wurde vom AUE-Vorstand akzeptiert. So wurden zu den Projektgruppensitzungen Vertreter aller Einrichtungen eingeladen.

Und wie lange lief das Projekt?

BC: Das Projekt nahm im Januar 1982 die Arbeit auf und endete mit der Abschlusstagung im Dezember 1984 an der Evangelischen Akademie in Loccum.

Wo war das Projekt angesiedelt?

Das Projekt war an der Geschäftsstelle des AUE in Hannover angesiedelt. Diese Geschäftsstelle war damals mit einem hauptamtlichen Geschäftsführer besetzt.

HV: Die wissenschaftliche Leitung hatte für das Projekt Horst Siebert von der Universität Hannover.

BC: Es waren schon damals zwei Leiter zentraler Einrichtungen im Vorstand des AUE, was sehr wertvoll für das Projekt war. Es handelte sich um Peter Faulstich als Leiter der Einrichtung in Kassel und Gerd Michelsen als Leiter der Einrichtung in Hannover. Es gab also eine gute Verbindung zwischen der Projektgruppe und dem Vorstand des AUE.

Wann entstand die Idee, im Rahmen des AUE regelmäßig Veranstaltungen für die Einrichtungen zu organisieren, und wann ist aus dieser Projektgruppe während der Laufzeit die AG-E gegründet worden?

BC: Die spätere AG-E hatte damals den Namen „Ständige Kommission der Zentraleinrichtungen“. Diese wurde auf Initiative der Projektgruppe durch Beschluss des AUE-Vorstandes 1983 gegründet. Ihr Vorläufer war seit 1981 die Arbeitsgruppe für Weiterbildung an Hochschulen als Beirat für das Kontaktstellenprojekt.

Waren schon die unterschiedlichen Hochschultypen - also Fachhochschulen und Universitäten - vertreten?

HV: Von 28 Zentraleinrichtungen waren im Jahr 1983 drei an Fachhochschulen angesiedelt und projektbeteiligt. Die FH Wiesbaden gehörte dazu.

Vielleicht kommen wir ja später noch mal auf die Fachhochschulen zurück. Ich habe den Eindruck, dass in den letzten 16 Jahren, in denen ich bei der DGWF aktiv bin, die Beteiligung der Fachhochschulen gegenüber früher sehr gestiegen ist. Jetzt aber erst mal wieder zum Ursprung. Was war die Motivation, eine solche Arbeitsgruppe der Einrichtungen - die AG-E - in dem Verband des AUE zu gründen?

BC: Die Motivation ist im Rahmen dieses Kontaktstellenprojektes entstanden. Die wissenschaftliche Weiterbildung war 1981 noch relativ jung, und es gab nur wenige Einrichtungen an Hochschulen. Wenn also im Projektverlauf anfangs 16 und später 28 Einrichtungen beteiligt waren, konnte man sich bei den Treffen in einen Seminarraum setzen. Das Kontaktstellenprojekt bot nun zum ersten Mal die Chance zum 
kontinuierlichen Austausch aller Einrichtungen untereinander. Nach meiner Erinnerung traf man sich ein- bis zweimal im Jahr, hat den Fortschritt des Projektes diskutiert, aber auch relativ bald, noch im Rahmen des Projektes, auch andere Fragen diskutiert, die die Einrichtungen interessierten. Da ging es z. B. darum:

- Was machen wir inhaltlich, konzeptionell?

- Wie ist in den einzelnen Bundesländern der rechtliche Rahmen?

- Wie ist der organisatorische Rahmen?

- Wo ist die Einrichtung in der Hochschule angesiedelt?

- Wie sind die Einrichtungen finanziert und ausgestattet?

- Welches sind die Perspektiven für die Weiterbildung an den jeweiligen Hochschulen und in den Bundesländern?

Das waren zwar Fragen, die auch in dem Projekt behandelt wurden, die wir aber unabhängig davon untereinander diskutieren wollten.

Also war das Anliegen der AG-E von Anfang an, sich auszutauschen, voneinander zu lernen und sich zu informieren, welche Inhalte und Schwerpunkte die einzelnen Einrichtungen haben?

BC: Auf jeden Fall. Im Grunde ist das Kontaktstellenprojekt auf ein vorhandenes Interesse gestoßen, das virulent war und schnell eine Eigendynamik entwickelte. Im Verlauf des Projektes wurde schnell klar: Das wollen wir nach Ablauf des Projektes nicht einstellen. Wir wollen diese Treffen fortsetzen.

Wie ist das im Vorstand des AUE aufgenommen worden - denn es war ja ein AUE-Projekt?

BC: Ich denke, das wurde im Vorstand unterschiedlich gesehen: Es gab Peter Faulstich und Gerd Michelsen aus Kassel und Hannover, die Mitglieder im AUE-Vorstand waren. Das heißt, die Einrichtungen waren durch sie im AUE-Vorstand vertreten. Aber der geschäftsführende Vorstand war noch in der Tradition der Gründung des AUE, d.h. Hochschullehrer waren in der Regel Vorsitzende und stellvertretende Vorsitzende - z. B. Joachim Dikau und Detlef Kuhlenkamp. Es gab eine lange Phase, in der der AUE sehr stark konzeptionell gearbeitet hat. Das wird deutlich, wenn man sich die Liste der Veröffentlichungen aus den Jahren seit der Gründung 1971 anschaut. Diese Veröffentlichungen aus dem Vorstand waren sehr wichtig, damit die Organisation ein inhaltliches und konzeptionelles Gerüst hatte und die Mitglieder des AUE sich damit auseinandersetzen konnten. Mit dem Kontaktstellenprojekt wurde zum ersten Mal - etwas flapsig gesagt - die Praxis der Mitglieder in den AUEVorstand getragen. Und zwar in Form der Erhebungen - was machen denn eigentlich diese Zentraleinrichtungen -, und damit auch das Interesse transportiert, sehr aktuelle Aspekte zu diskutieren in Ergänzung der bis dahin eher theoretischen Diskussionen. Diese Interessen von der praktischen Seite wurden nicht unbedingt mit großem Wohlwollen begrüßt. Aber es war gut vorbereitet: Gegen Ende des Kontaktstellenprojektes entwarf eine Kommission ein Papier, um deutlich zu machen, dass die Beteiligten dieser Arbeitsgruppe einen kontinuierlichen Austausch brauchen 
und wir uns innerhalb des AUE konstituieren möchten. 1983 wurde dann durch Vorstandsbeschluss des AUE die "Ständige Kommission der Zentraleinrichtungen“ als offizielle Einrichtung des AUE, als Untergliederung mit einem Sprecherrat, gegründet. Von 1983 an hat dann diese Kommission, die sich später AG-E nannte, regelmäßig Tagungen durchgeführt.

Gab es damals schon die zentrale Jahrestagung des AUE und zusätzlich die Tagungen der $A G-E$ ?

BC: Die Jahrestagungen sind eine sehr alte Einrichtung, die es bald nach Gründung des AUE regelmäßig gab. Die AG-E hat dann kontinuierlich von 1983 an jedes Jahr eine eigene Tagung durchgeführt. Um nicht in Terminkollision zu kommen, wurde diese Tagung in der ersten Jahreshälfte geplant, da die Jahrestagungen des AUE/ DGWF immer im September stattfanden. Deshalb hat sich bald der Terminus „Frühjahrstagung" etabliert. Es war also die zweite Tagung des AUE, obwohl sie einen völlig anderen Charakter hatte, sowohl von den Thematiken her als auch von der Zielgruppe.

Helmut, wie war das mit den anderen beiden Sektionen?

HV: Die BAG WiWA bestand seit 1984 schon vor ihrer Integration in den AUE als nicht verfasste Gemeinschaft und brachte ihre jährlichen Tagungen als „Morgengabe“ im Jahr 1994 mit in den Verein ein. Sie wurden zeitlich an den AUE-Rhythmus angepasst und finden seither auch als Frühjahrstagungen statt. Bei der AG-F gab es jährliche Tagungen seit der Gründung 1995, die von Anfang an auch im Frühjahr durchgeführt wurden.

Zurück zu den Tagungen der AG-E. Sie waren und sind ein zentraler Arbeitsschwerpunkt $\operatorname{der} A G-E, d . h$. Themen zu überlegen, die Veranstaltungen vorzubereiten. Wie sind diese Themen zustande gekommen und inwieweit waren sie auch abhängig von aktuellen bildungspolitischen Fragen?

BC: Die Themenfindung fand immer innerhalb der Kommission statt. Es gab schon so eine Art Mitgliederversammlung, obwohl das alles lange Zeit gar nicht formell geregelt war. In diesem Kreis wurde auch besprochen, welche weiteren Themen angepackt werden sollten; Themen, die stark durch die Arbeit der Zentraleinrichtungen geprägt waren. Die Bezeichnung „Zentraleinrichtungen“ hat man später nicht mehr verwendet, weil sich im Laufe der Zeit auch andere institutionelle Formen der Weiterbildung an Hochschulen entwickelten. Hier ein paar Beispiele: 1984 wurde in Frankfurt das Thema „Informations- und Kommunikationstechniken als Herausforderung für wissenschaftliche Weiterbildung“ behandelt, 1985 in Osnabrück „Das Berufsbild der Leiter und Mitarbeiter von Zentraleinrichtungen“, 1986 in Karlsruhe "Die Entwicklung der hochschulgetragenen Weiterbildung in Baden-Württemberg“. Zentrale Fragen waren, wie entwickelt sich die Arbeit der Einrichtungen vor Ort, wie entwickelt sich die Organisation usw. Die übergeordneten bildungspolitischen Themen waren Gegenstand der Jahrestagungen und des Vorstandes. 
Haben sich daraus auch wieder Themen der AG-E abgeleitet?

BC: Es gab bestimmte Einschnitte. Der erste Einschnitt, bei dem die AG-E auf einer mehr bildungspolitischen Ebene diskutiert hat, waren die beiden Tagungen 1991 und 1992 nach der Wiedervereinigung.

1991 lautete das Thema in Weimar „Weiterarbeit der wissenschaftlichen Weiterbildung in Deutschland“. Die große Frage war, was machen wir mit den beiden Einrichtungsformen in West- und Ostdeutschland? Gibt es einen Weg, diese zusammenzuführen, und wie soll der Weg inhaltlich gestaltet sein? 1992 war das Thema noch pointierter: „Weiterbildung an Hochschulen als kulturelle Aufgabe im Rahmen der Vereinigung“. Die Frage war, wie gehen diese unterschiedlichen Kulturen, die da aufeinandertreffen, miteinander um und wie finden sie einen gemeinsamen Weg?

HV: Auf Verbandsebene bildete sich in den neuen Bundesländern zunächst eine selbstständige Organisation, der Arbeitskreis Universitäre Weiterbildung (AUW). AUE und AUW arbeiteten aber von Anfang an eng zusammen. Zum 01.01.1991 kam es schließlich zu einer Integration der beiden Verbände, die zugleich einer der Ausgangspunkte für eine größere Reorganisation darstellte. Diese mündete im Jahr 2003 in die Namensänderung zur Deutschen Gesellschaft für wissenschaftliche Weiterbildung und Fernstudium (DGWF) und in andere bedeutsame Satzungsänderungen.

BC: Ein zweiter Einschnitt war der Bologna-Prozess. Im Zuge der Umstellung auf die Bachelor- und Master-Struktur ergab sich erstmalig die Möglichkeit, in der Weiterbildung abschlussbezogene Studiengänge anzubieten, die Weiterbildenden Masterstudiengänge. In dieser Zeit wurde diese bildungspolitische Debatte auch stark in der AG-E geführt.

Das hatte auch ganz praktische Konsequenzen für die Einrichtungen, nämlich inwieweit beteiligen sie sich an diesen Weiterbildungs-Studiengängen der Fachbereiche? Welche Rolle spielten zum Beispiel die Zertifikate im Rahmen des Bologna-Prozesses?

BC: Der Bologna-Prozess hatte in mehrfacher Hinsicht Auswirkungen auf die Einrichtungen. Durch die Einführung der beiden neuen Abschlussformen Bachelor und Master kam insgesamt Bewegung in die Hochschulen im Hinblick auf Abschlüsse und was das Weiterstudieren nach dem Bachelor-Abschluss anbelangt. Die Frage, was und wie man an Hochschulen noch weiter studieren kann, stellte sich durch diese neue Studienstruktur ganz neu - natürlich auch durch die Einführung des Weiterbildungs-Masters. Das war eine deutliche Herausforderung für die wissenschaftliche Weiterbildung und ihre traditionellen Angebotsformate, etwa die Zertifikatsangebote.

Bernhard, du hattest vorhin erwähnt, dass die Tagungen des AUE im Herbst zu bestimmten bildungspolitischen Themen anders abliefen als die kleineren Frühjahrstagungen der AG-E. Kannst du zu den didaktischen Konzepten etwas sagen? Und wie war damals die Beteiligung - waren das eher die Leitungen der Einrichtungen, die sich getroffen haben? 
BC: Es gab und gibt zwischen den AG-E-Tagungen und der Haupttagung des Verbandes klare Unterschiede. Der Schwerpunkt der Jahrestagung des Verbandes liegt auf wichtigen bildungspolitischen Entwicklungen, angefangen mit der Entwicklung der ehemaligen Bundesrepublik, über das vereinte Deutschland bis hin zu Europa. Es gab aber auch Themen, die der Gesamt-Verband in Kooperation mit den Verbänden in Österreich und der Schweiz sowie auch mit EUCEN, dem europäischen Verband gemeinsam bearbeitet hat. Damit war der potenzielle Teilnehmerkreis, der von den Tagungsthemen angesprochen wurde, ein viel größerer. Es gab und gibt auf den Jahrestagungen einen deutlichen Anteil von Teilnehmenden, die nicht Verbandsmitglied sind. Es sind aber auch andere Verbände, andere Träger und Institutionen eingeladen, auf den Jahrestagungen mit der DGWF diese bildungspolitischen Fragen zu diskutieren. Das war und ist ein besonderes Ziel.

Demgegenüber sind die AG-E-Tagungen eher verbandsintern. Sie sind begründet als Zusammenkunft der Einrichtungen, und damit sind die Leitungen und die Mitarbeiterinnen und Mitarbeiter diejenigen, die vorrangig angesprochen sind.

\section{Organisation und Mitgliedschaft in der AG-E}

Ich möchte jetzt einen Blick auf die Organisation und Mitgliedschaft der AG-E werfen. Wer kann Mitglied in der AG-E werden?

BC: Laut Geschäftsordnung, die 2001 verabschiedet wurde, sind die Mitglieder der AG-E die Einrichtungen für Weiterbildung oder auch weiterbildende Studiengänge, die unabhängig von einer Weiterbildungseinrichtung an Hochschulen organisiert werden, sowie die hochschulnahen Einrichtungen, die als e. V., GmbH usw. organisiert sind. Man muss als Einrichtung bzw. als Hochschule Mitglied in der DGWF sein, damit man in der AG-E mitarbeiten kann. Die AG-E ist quasi eine Interessenvertretung der Einrichtungen für Weiterbildung innerhalb der DGWF.

Ihr habt sehr lange erfolgreich die Tagungen organisiert und inhaltlich gearbeitet - wie habt ihr 2001 die Geschäftsordnung begründet? Warum brauchte man jetzt eine Geschäftsordnung? Worin lag der Vorteil für die Mitglieder der AG-E, so eine Geschäftsordnung zu haben?

BC: Der Grund für die Geschäftsordnung, die 2001 erarbeitet und dann vom Vorstand verabschiedet wurde, war ganz banal: Die anderen beiden Arbeitsgemeinschaften, die BAG WIWA und die AG-F hatten eine Geschäftsordnung. So kam es, dass die AG-E auch eine Geschäftsordnung haben sollte, damit alles einheitlich geregelt war. Also haben wir ähnliche Regelungen für die AG-E formuliert und dem Vorstand vorgelegt, der sie dann verabschiedete. Wir haben von 1983 bis 2000 sehr gut ohne Geschäftsordnung gearbeitet. Wir wählten einen Sprecherrat und haben auch quasi Mitgliederversammlungen durchgeführt, ohne dass es schriftlich fixiert war.

An dieser Stelle mal kurz zurück zu der Frage der Beteiligung der Fachhochschulen: Die ständige Kommission der Zentraleinrichtungen wurde 1987 umbenannt in „Ar- 
beitsgruppe der Einrichtungen für Weiterbildung an Hochschulen - AG-E“. Die Umbenennung wurde damit begründet, dass sich das Spektrum der Einrichtungen erweitert und sehr stark differenziert hatte. Es waren längst nicht mehr nur Zentraleinrichtungen, sondern es gab Referate beim Präsidium bzw. Rektorat oder einzelne Projekte und andere Organisationsformen der Weiterbildung an Hochschulen. Der zweite Grund war, dass die Fachhochschulen immer deutlicher anfingen, Weiterbildungsangebote zu entwickeln. Deshalb war der Begriff „Ständige Kommission der Zentraleinrichtungen" nicht mehr angemessen. Man hat dann auch nicht mehr nur von Universitäten gesprochen, sondern „Arbeitsgruppe der Einrichtungen an Hochschulen“, sodass auch andere Hochschultypen einbezogen waren.

Die Fachhochschulen haben sich ja erst in den 7oer-Jahren gegründet, sodass sie auch eine kürzere Tradition mit der Entwicklung von Weiterbildung haben als Universitäten.

Für die Mitglieder der AG-E war die Geschäftsordnung eigentlich zweitrangig. Das zeigt, mit welcher Motivation die Mitglieder der AG-E sich eingebracht haben. Immer wenn die Motivation hoch ist, wird eine Geschäftsordnung nur für bestimmte Rahmenverabredungen erforderlich und spielt in der Zusammenarbeit nur eine sehr untergeordnete Rolle. Ich finde es heute allerdings praktisch, dass es eine Geschäftsordnung gibt, weil sich die Beteiligung an der AG-E im Laufe der Zeit auch verändert. Wir haben inzwischen durch die zahlreichen Projekte wie zum Beispiel "Offene Hochschule" viele junge Leute, die in die AG-E dazugekommen sind. Diese jungen Leute sind oft hoch motiviert mitzuarbeiten. Dafür ist es gut, so einen gemeinsamen Rahmen zu haben.

BC: Spontan fallen mir drei Aspekte ein, an denen es sich zeigt, dass es sinnvoll ist, eine Geschäftsordnung zu haben:

1. Wer kann Mitglied in der AG-E sein, auch in direktem Zusammenhang mit der Mitgliedschaft im DGWF?

2. Die Wahl des Sprecherrates - wer kann das sein?

3. und die Frage, welche Aufgaben hat die AG-E und welche Ziele verfolgt sie?

Hast du in der Organisationsform und der Mitgliedschaft eine Veränderung im Laufe der Jahre feststellen können?

BC: Es gab eine ganz wesentliche Veränderung, und das ist die Größe der AG-E und der DGWF insgesamt. Die Beteiligung der Hochschulen an der wissenschaftlichen Weiterbildung ist deutlich gewachsen. Wenn man bedenkt, dass 1983 gerade einmal 28 Einrichtungen in einer Totalerhebung untersucht wurden, liegt die Zahl der Einrichtungen heute in der Größenordnung des Zehnfachen. Das betrifft also die quantitative Entwicklung. Wir hatten 1994 eine Tagung in Rostock mit dem Titel: „Leistungsspektren zentraler Einrichtungen für Weiterbildung“. Es ging vor allem um den Austausch und die Selbstvergewisserung: Was machen wir eigentlich in unseren Einrichtungen? Die Tagung in Rostock fand mit rund 6o Teilnehmenden statt. Zehn Jahre später wurden 100 bis 120 Teilnehmende erreicht. Das war eine wesentliche Veränderung, die Auswirkungen hatte auf die Organisation der Tagungen und auf den Informationsaustausch zwischen den Tagungen. 
HV: Hier sollte man nicht unerwähnt lassen, dass vor einigen Jahren durch die Satzungsreform die Vorsitzenden der Sprecherräte der DGWF-Sektionen zu ordentlichen Mitgliedern des Vorstandes aufrückten. Zunächst wurden sie nur im Zusammenhang mit sie betreffenden Tagungsordnungspunkten eingeladen. Danach bekamen sie einen ständigen Sitz im Vorstand und nach der o. e. Satzungsreform auch eine Stimme.

\section{Ziele und Konzeption der AG-E}

In der Geschäftsordnung sind auch die Ziele formuliert. Daran können sich gerade neue Mitglieder der AG-E orientieren.

BC: Die Formulierung der Ziele war 2001 nur eine Fixierung dessen, was die AG-E schon mehr als 15 Jahre gemacht hat.

Mit der Anzahl der Mitglieder in der AG-E kam auch eine deutliche Differenzierung der Themen und Fragestellungen, die die sehr heterogene Mitgliedschaft mit sich brachte.

In der Geschäftsordnung sind die Ziele so formuliert:

- „eine Plattform zu bilden für die Diskussion aller theoretischen und praktischen Dimensionen der Weiterbildung an Hochschulen,

- das allgemeine Verständnis für die Hochschulweiterbildung zu fördern,

- eine Infrastruktur aufzubauen, zu pflegen und weiterzuentwickeln für die institutionsübergreifende Entwicklung, Verbreitung und Qualifizierung von Angeboten der wissenschaftlichen Weiterbildung,

- Forschung und Entwicklung zur Weiterbildung an Hochschulen zu initiieren und zu fördern und sich an entsprechenden Projekten zu beteiligen,

- die Weiterbildung an den Hochschulen Deutschlands auf europäischer und internationaler Ebene gemeinsam zu vertreten und an übernationalen Projekten zu University Continuing Education (UCE) teilzunehmen.“

Die Frühjahrstagungen waren und sind eng mit diesen Zielen verbunden. Die DGWF/ der AUE haben zahlreiche Empfehlungen herausgebracht. Mir sind die Empfehlungen "Perspektiven wissenschafticher Weiterbildung in Deutschland aus der Sicht der Einrichtungen an Hochschulen" präsent, die 2005 in Wien verabschiedet wurden. Bernhard, kannst du etwas sagen, wie es zu den Empfehlungen von 2005 gekommen ist?

BC: Es gab im Vorfeld der genannten Empfehlungen eine Diskussion über eine Vergewisserung darüber, was sich bildungspolitisch verändert hat und in Veränderung war. Und umgekehrt war die Frage, wofür steht eigentlich die DGWF in dieser bildungspolitischen Landschaft? Es ging um eine neue Standortbestimmung und Selbstvergewisserung. Die Empfehlungen von 2005 haben einen starken Kern, was Aufgabe und Struktur, Ausstattung und Finanzierung der Einrichtungen sein sollte. Aber das ist eingebettet in einen klar formulierten bildungspolitischen Rahmen. Es 
gibt einen langen Vorspann einer bildungspolitischen Einschätzung der Situation in Deutschland und zum Teil in Europa und die Frage, was daraus für die wissenschaftliche Weiterbildung folgen soll, inhaltlich, organisatorisch und im Hinblick auf die Ausstattung der Einrichtungen. Diese Empfehlungen wurden sehr breit im Verband diskutiert. Die Arbeitsgemeinschaften waren daran beteiligt, weil sie durch ihre Sprecherinnen und Sprecher wie von Helmut gerade beschrieben im Vorstand vertreten waren. Ich kann mich auch erinnern, dass dieser Entwurf sehr intensiv mit dem Beirat der DGWF diskutiert wurde. Danach wurde der Entwurf nochmals überarbeitet und in Wien verabschiedet.

Da spielte der Bologna-Prozess in der Zeit, in der die Umsetzung im vollen Gange war, eine Rolle. Die Frage war, was hat Bologna mit der wissenschaftlichen Weiterbildung zu tun?

BC: Ja genau. Es war klar, dass der Bologna-Prozess und der beginnende Prozess der Neuorganisation der Hochschulen - Stichwort Managementprozesse in die Hochschulen hineinzutragen - sehr stark diskutiert und auch in die Empfehlungen einbezogen wurde.

Diese Empfehlungen hatten sicher auch Einfluss auf die alltägliche Arbeit in den Einrichtungen. Sie konnten als Orientierung und Handwerkszeug für die eigene Einrichtung genutzt werden. Es wurden in der Zeit auch neue Einrichtungen aufgebaut oder neu ausgerichtet. Ich selbst habe diese Empfehlungen - auch die, die später noch entstanden sind, als sehr hilfreich für meine Arbeit erlebt und natürlich für die Personen, die neu in eine Einrichtung kommen.

BC: Ja, es kamen neue Einrichtungen hinzu und Einrichtungen sind gewachsen. Insofern waren diese Empfehlungen hilfreich als Handreichung. Sie sind aus der Perspektive der AG-E in den Diskussionen vorbereitet worden. Das sieht man auch an den Themen der Frühjahrstagungen in den Jahren davor, so z. B. 2002 in Münster "Qualitätssicherung und Akkreditierung wissenschaftlicher Weiterbildung“. 2003 diskutierten wir in Halle das Thema „Aktuelle Entwicklungen in der wissenschaftlichen Weiterbildung" und 2004 in Mainz lautete das Thema "Wissenschaftliche Weiterbildung im Umbruch - Rolle und Aufgabe der Einrichtungen für Weiterbildung“. Alles das sind Aspekte, die in die Empfehlungen von 2005 einflossen.

Es gab ja noch andere Empfehlungen, die auch für die AG-E und für die Einrichtungen relevant waren. Welche Empfehlung könntest du noch hervorheben?

BC: Es gab 2010 die Empfehlungen „Formate wissenschaftlicher Weiterbildung“. Aus der Mitgliederschaft der AG-E heraus entstand die Anforderung, zu der Vielfalt der Formate und deren Bedeutung und ganz konkreten Ausgestaltung Eckpunkte zu fixieren. Zu klären war, welche unterschiedlichen Formate gibt es, welche Bedeutung und Funktion haben sie jeweils, und wie ist das in den einzelnen Bundesländern geregelt, wie sind die traditionellen Formate im Verhältnis zum weiterbildenden Master $\mathrm{zu}$ sehen. Das Motiv war, etwas Licht in dieses Dickicht hineinzubringen. Eine kleine Gruppe aus dem AG-E-Sprecherrat hat diese Empfeh- 
lungen entworfen und sie dem Vorstand vorgelegt. Dort wurden sie dann verabschiedet. Auch für diese Empfehlungen gilt, dass es nicht möglich war, angesichts der Heterogenität in der Weiterbildungslandschaft an den Hochschulen eindeutige Empfehlungen auszusprechen. Es gab schon in dem Kontaktstellenprojekt die flapsige Bemerkung „Es gibt so viele Modelle wie es Einrichtungen gibt“; im Grunde genommen hat sich das kaum verändert. Das hat auch damit zu tun, dass durch die Gesetzgebung in den Bundesländern unterschiedliche rechtliche Rahmenbedingungen bestehen.

Es gibt also auch 2016 noch kein Patentrezept für die Einrichtungen, genau so muss eine Einrichtung mit ihren Inhalten und Organisationsformen aussehen. Dennoch sind die Empfehlungen Orientierung für die Verantwortlichen in den Einrichtungen, aber auch für Hochschulleitungen, die Einrichtungen neu aufbauen oder umstrukturieren wollen. Das bezieht sich auch auf die Empfehlungen der Formate.

BC: Die Empfehlungen für die Formate hatten orientierenden Charakter. Mit der Diskussion, dass es mit dem Weiterbildungs-Master eine Weiterbildung mit einem akademischen Abschluss gibt, kam die Frage auf, ob man die anderen Formate unterhalb des Abschlusses noch braucht. Es wurde nach den Zielgruppen gefragt, die wissenschaftliche Weiterbildungsangebote nachfragen. Eine zentrale Rolle hat dabei auch die Diskussion um die Zertifikate gespielt. Mit der Übersicht der Formate wurde gleichzeitig die Sinnhaftigkeit dieser verschiedenen Formate erläutert. Und noch weiter gedacht: Können diese Formate unterhalb des Masters mit der alten Idee einer Baukasten-Hochschule verbunden werden? Wenn es gelingt, diese Formate anrechenbar zu machen und mit Leistungspunkten zu versehen, können sie Bausteine sein für einen irgendwann kumulativ zu erreichenden Hochschulabschluss. Das ist bildungspolitisch eine ganz wichtige Idee, obwohl sie leider wenig umgesetzt wird.

Ich glaube, das braucht wieder Zeit, bis sich die Empfehlungen der Formate in der Umsetzung neuer Weiterbildungsangebote realisieren lassen. Sie müssen aber auch weiterentwickelt werden. Ich bin sicher, das Baukastensystem wird in den nächsten Jahren eine Bedeutung erhalten. Das ist ein klassisches Thema der Handlungsfelder, die sich die AG-E mit ihren Zielen in ihrer Geschäftsordnung gegeben hat. Könntest du darauf noch mal eingehen?

Es gab 2001 herum eine Diskussion über die Ziele und Handlungsfelder der AG-E. Es wurden drei Handlungsfelder formuliert:

1. Erfahrungsaustausch: eine Plattform der Diskussion theoretischer und praktischer Fragestellungen der Weiterbildung an Hochschulen zu schaffen. Das wird durch die Tagungen jährlich aufgegriffen.

2. Kooperation: d.h. die Einrichtungen, da wo es sinnvoll ist, miteinander zu verflechten regional und überregional. Da gibt es ja erfolgreiche Beispiele wie in Hessen. Ich meine damit das Netzwerk WissWeit. Es geht darum, eine Infrastruktur aufzubauen, um Möglichkeiten für gemeinsame Projekte oder auch andere Fragestellungen gemeinsam zu bearbeiten. 
3. Präsenz nach innen und außen: Die AG-E will sich zeigen und für sich selbst Werbung machen und damit auch das Verständnis für die Weiterbildung an Hochschulen fördern, innerhalb des Verbandes, aber auch nach außen. Dieses Handlungsfeld ist nie sehr stark bearbeitet worden. Letztendlich hat man dem Hauptverband diese Arbeit weiterhin zugestanden, mit dem er sich ansprechend präsentieren kann.

HV: Es haben sich im Laufe der Jahre flächendeckend Landesgruppen gegründet, die durch ihre Vorsitzenden inzwischen auch dem Vorstand angehören. Das ist aber noch ein Thema für sich.

Das dritte Handlungsfeld war für die AG-E in den letzten Jahren mit dem Thema Marketing besonders relevant. Wir haben 2014 die Tagungen in Konstanz und 2015 Zürich rund um das Thema gestaltet. Die AG-E hat mit den Kolleginnen und Kollegen darüber diskutiert, wie wir uns als Einrichtungen besser nach innen und außen präsentieren und darstellen können, sodass wir eine größere Relevanz in der eigenen Organisation und auf dem Weiterbildungsmarkt erhalten. Es geht auch darum, wie erfahren eigentlich Menschen im Berufvon den Angeboten der wissenschaftichen Weiterbildung an Hochschulen.

BC: Selbstverständlich. Im Grunde genommen geht das ineinander über, dass man als AG-E sagt, wir müssen uns selbst stärker präsentieren, um bekannt zu werden, und die einzelnen Einrichtungen müssen ihre Existenz und ihre Programme bewerben. Deshalb war auch die Frage des Marketings schon früh innerhalb der AG-E ein Thema.

\section{Highlights aus den Tagungen der AG-E}

Am Schluss will ich euch fragen, ob es für euch ein Highlight gibt, von dem ihr noch berichten wollt?

BC: Ja, für mich gibt es zwei Punkte. Es ist ja vorhin die Frage von dir gewesen, wie die Tagungen didaktisch aufgebaut waren. Die Mehrzahl der Tagungen sind sehr klassisch abgelaufen, damit meine ich Vortrag, Diskussion und wieder Vortrag. Aus der Mitgliedschaft kam dann der Wunsch auf, der Diskussion und damit dem Erfahrungsaustausch der Teilnehmenden mehr Raum zu geben. Das wurde berücksichtigt. Man bildete auf den Tagungen Arbeitsgruppen zu Teilthemen. So sollten die Tagungen didaktisch aufgelockert werden. Einige wenige Tagungen waren didaktisch komplett anders organisiert. Das waren Highlights. Dazu gehörte die Zukunftswerkstatt mit dem Titel „Zukunftsfähige wissenschaftliche Weiterbildung“ 1997 in Berlin. Diese Zukunftswerkstatt wurde von zwei externen Moderatoren gestaltet.

HV: Die Ausgangsfrage lautete „Was fördert oder behindert meine Bemühungen, an meiner Hochschule eine zukunftsfähige wissenschaftliche Weiterbildung dauerhaft zu etablieren?“ Diese Tagung kann ich in inhaltlicher und didaktischer Hinsicht und auch von den Ergebnissen wirklich als eines der Highlights nennen. 
BC: 2007 hatten wir einen sogenannten Strategie-Workshop der AG-E in Cottbus mit dem Titel: „Bologna-Prozess, Hochschulentwicklung und wissenschaftliche Weiterbildung“. Dieser Workshop wurde ebenfalls durch einen externen Moderator begleitet. Die Gruppenarbeit stand dabei im Zentrum, dort wurde zu vier Themen gearbeitet, die durch kurze Input-Referate eingeleitet waren. So konnten wir die Erfahrung der Teilnehmenden intensiv nutzen.

In den letzten vier Jahren hatten wir die Frühjahrstagungen der AG-E jeweils als moderierte Großgruppenkonferenz mit externer Moderation gestaltet und damit sehr gute Erfahrungen gemacht. Durch diese Didaktik wurde der Austausch gefördert und die Kompetenz der Teilnehmenden in den Mittelpunkt gestellt.

Herzlichen Dank für das Gespräch! 


\title{
Zur Entstehungsgeschichte der Arbeitsgemeinschaft für das Fernstudium an Hochschulen (AG-F)
}

\author{
BURKHARD LEHMANN ${ }^{1}$
}

Die Gründung der „Arbeitsgemeinschaft für das Fernstudium an Hochschulen“ (AG-F) erfolgte 1995 als eigenständige Institution. Nur wenige Monate nach erfolgter Gründung wurde sie zu einer von drei Sektionen des „Arbeitskreises Universitäre Erwachsenenbildung“ (AUE) ${ }^{2}$. Ins Leben gerufen wurde die AG-F von Vertretern regionaler Studienzentren der FernUniversität Hagen (FeU), d.h. den Zentren Bremen, Oldenburg, Karlsruhe, Saarbrücken, Koblenz, Kaiserslautern, Lüneburg, Hamburg, Frankfurt/Main, Hildesheim und Kassel. ${ }^{3}$ Unter institutionellem Blickwinkel betrachtet, war die Errichtung der Arbeitsgemeinschaft das Ergebnis einer Initiative von Fernstudien-Serviceprovidern - man kann auch sagen: von Zulieferern für das akademische Fernstudium in Deutschland. Das Gründungsjahr der AG-F fällt in eine Zeit des Fernstudienaufbruchs. Dieser Aufbruch beschreibt die zweite Phase der Fernstudienentwicklung in Deutschland, die in einer Diversifizierung und Ausweitung des akademischen Fernstudiums bestand. Die erste Fernstudienphase ${ }^{4}$ fällt mit der 1975 ins Leben gerufenen FernUniversität zusammen, die bis heute das „Leading House des deutschen Fernstudiums“ ist. Das gilt insbesondere für die beeindruckende Zahl von Fernstudierenden, die sich an dieser Hochschule immatrikuliert haben. Mit 77.000 Studierenden (WS 14/15) ist sie nicht nur Deutschlands größte Universität, sondern zugleich auch bundesweit der zahlenmäßig größte Fernstudienanbieter. ${ }^{5}$

1 Ich danke den Kolleginnen und Kollegen für die kritische Durchsicht des Beitrages (Herrn Iberer, Frau Stamm, Herrn Vogt). Insbesondere danke ich Otto Peters für seine wertvollen Ergänzungen und die lebendige Diskussion.

2 Die beiden anderen Sektionen heißen: „Arbeitsgruppe zentrale Einrichtungen für Weiterbildung an Hochschulen“ (AG-E) und „Bundesarbeitsgemeinschaft wissenschaftliche Weiterbildung Älterer“ (BAG WIWA).

3 Die an der Gründungsversammlung beteiligten Akteure waren: Ernst Raters (Bremen), Hans-Henning Kappel (Frankfurt/Main), Helmut Vogt (Hamburg), Erwin Wagner (Hildesheim), Burkhard Lehmann (Kaiserslautern), Joachim Klaus (Karlsruhe), Helmut Zimmermann (Kassel), Joachim Loeper (Koblenz-Landau), Jan Wellendorf (Lüneburg), Ulrich Bernath (Oldenburg) und Heinz Augenstein (Saarbrücken).

4 Dass das nicht-akademische Fernstudium gelegentlich auch als Fernlehre oder Fernunterricht bezeichnet, wird, bleibt hier unberücksichtigt. Der Vollständigkeit halber soll hier erwähnt werden, dass im Februar 1974 Bund und Länder ein Verwaltungsabkommen über den Modellversuch „Fernstudium im Medienverbund“ (FIM) abgeschlossen hatten, der allerdings nach allgemeiner Auffassung nicht erfolgreich verlief.

5 Im internationalen Vergleich gehört sie allerdings zu den kleineren Fernuniversitäten. 


\section{Neue Anbieter}

Der FernUniversität gebührt unzweifelhaft das Verdienst, dass sie das akademische Fernstudium in Deutschland hoffähig gemacht hat und eine Vielzahl von Vorurteilen gegenüber dem Fernlehrsystem beseitigen konnte, auch wenn es bis heute immer noch Ressentiments gibt. Jahrelang war die Hagener Universität die einzige Hochschule ihrer Art und damit der unangefochtene Platzhirsch. Das änderte sich erst 1981, als mit der „Fernhochschule für Berufstätige“ (AKAD) die erste (private) Fern-Fachhochschule in den Bildungsmarkt eintrat. Ihr folgten auf staatlicher Seite eine Reihe von Fernfachhochschulverbünden. So wurden 1993 die „Verbundstudien NRW“ (Hagen), 1994 die „Zentralstelle für Fernstudien an Fachhochschulen“ (Koblenz) und im gleichen Jahr der „Fernstudienverbund der Länder“ gegründet, aus dem der „Hochschulverbund Distance Learning“ (HDL) als Nachfolgeorganisation hervorgegangen ist. Auf privatwirtschaftlicher Ebene folgten 1997 die Errichtung der „Wilhelm Büchner Hochschule“ in Darmstadt sowie die „Hamburger Fernhochschule“, 2003 die „Europäische Fernhochschule“ (Hamburg) und 2005 die „Apollon Hochschule“ mit Sitz in Bremen.

\section{Historische Entwicklung}

Der entscheidende Impuls zur Diversifizierung und Ausweitung des Fernstudiums in Deutschland ging von den „Empfehlungen des Wissenschaftsrates zum Fernstudium“ (1992) aus. Diese Empfehlungen waren vor dem Hintergrund der Wiedervereinigung der beiden deutschen Staaten entstanden. Sie lieferten den Anlass zur Inspektion der Fernstudiensituation, da nicht nur in der alten Bundesrepublik ein Fernstudienwesen existierte, sondern ein solches auch in der ehemaligen DDR bestanden hatte. Beide Systeme waren jedoch in didaktischer und organisatorischer Hinsicht verschieden. In den alten Bundesländern (BRD) gab es unterhalb des Hochschulniveaus das kommerzielle Korrespondenzfernstudium, während sich in der ehemaligen DDR ein Konsultationsfernstudium ${ }^{6}$ nach dem Vorbild und Muster des sowjetischen Fernstudiums etabliert hatte (vgl. auch Schwier 1962; Pfundtner 1995). Die vom Wissenschaftsrat vorgenommenen Analysen und Betrachtungen mündeten in der Einrichtung eines „Förderschwerpunktes Fernstudium“ bei der „Bund-Länder-Kommission für Bildungsplanung und Forschungsförderung“ (BLK), ausgestattet mit einem beachtlichen finanziellen Volumen zur Entwicklung von Fernstudienangeboten. Es ist diesem Förderschwerpunkt $\mathrm{zu}$ verdanken, dass sich die Zahl der Fernstudienangebote und Fernstudienanbieter kontinuierlich auszuweiten begann. Eine Folge davon war u.a., dass die FernUniversität ihre Monopolstellung einbüßte, die sie bis dahin unangefochten innehatte.

6 Das Konsultationsfernstudium zeichnete sich vor allem durch großzügige Freistellungszeiten seitens der Arbeitgeber zur Teilnahme am Fernstudium und seine umfangreichen Präsenzzeiten aus. 
Die Gründer der AG-F waren Akteure an der Schwelle des Übergangs von der ersten zur zweiten Phase der Fernstudienentwicklung. Als Teil des Hagener Betreuungssystems bildeten sie einen integralen Bestandteil des FernUniversitätssystems und den damit im Zusammenhang stehenden Aufgaben, die auf Basis von Kooperationsverträgen erfüllt wurden. Als externe und nicht unmittelbar dem Direktionsrecht der FeU unterliegende Dienstleister außerhalb ihres Sitzlandes verfügten die Zentren über Handlungs- und Gestaltungsspielräume, die sie aktiv nutzten. Es wurden eigene Ideen zum Fernstudium und seiner Organisation diskutiert, der Blick über den Tellerrand gewagt und auch die Fühler nach anderen Fernstudiensystemen wie etwa in den Niederlanden oder dem Vereinigten Königreich ausgestreckt. Das, was die Gründerzentren prägte und zur Auslösung des Gründungsimpulses beitrug, war vor allem das Bewusstsein, nicht ein bloßes Anhängsel der FernUniversität zu sein, sondern eine Fernstudieneinrichtung mit eigenständiger Fachkompetenz und Expertise. Einige der an der Gründung der AG-F beteiligten Zentren waren u.a. zu Nutznießern des „Förderschwerpunktes Fernstudium“ geworden und betrieben die Entwicklung von Fernstudiengängen in Eigenregie. Aus Fernstudienservice-Providern waren auf diese Weise Fernstudienproduzenten, -anbieter und -vermarkter geworden, die zugleich dem Stammhaus des Fernstudiums bis auf Weiteres verbunden blieben.

Der eigentliche Gründungsakt der AG-F fand im Anschluss an eine Fachtagung der Fernstudienzentrumsleiter in Oldenburg statt. Nachdem die Tagung zum Thema „Fernstudium in den USA“ mit Gary Miller vom „State College“ in Pennsylvania als Referent beendet war, wurde die nachfolgende Tagesordnung aufgerufen:

1. „Wahl eines Versammlungsleiters und eines Protokollführers

2. Festsetzung der Tagesordnung

3. Berichte, Mitteilungen, Informationen

4. Diskussion zur Frage des $\mathrm{Ob}$ und des Wie eines Verbundes für das Fernstudium an den Präsenzhochschulen

Falls das vorgeschlagene Modell (AUE-AG) konsensfähig ist:

5. Diskussion, Verabschiedung und ggf. Unterzeichnung der Geschäftsordnung

6. Wahl eines vorläufigen Sprecherrates

7. Weiteres Verfahren

8. Kohlfahrt

Falls das vorgeschlagene Modell nicht konsensfähig ist, wohl aber die Gründung eines Verbundes als notwendig erachtet wird.

9. Weiteres Verfahren

10. Kohlfahrt

Falls die Gründung eines Verbundes nicht als sinnvoll erachtet wird.

11. Kohlfahrt"

Die Tagesordnung signalisiert, dass man auf alle Eventualitäten vorbereitet und keineswegs sicher war, dass die AG-F gegründet werden würde. Fest stand nur, dass die 
Veranstaltung - wie der Tagesordnung zu entnehmen ist - in einem norddeutschen Lokal-Kolorit, der Kohlfahrt münden würde.

Das Fragezeichen hinter dem „AUE-AG-Modell“ war konzeptionellen Erwägungen geschuldet. Schließlich musste erwogen werden, ob eine in Aussicht genommene Interessenvertretung des Fernstudiums mit den Belangen einer an der Weiterbildung an Hochschulen ausgerichteten Organisation harmoniert. Bekanntlich hatte sich der „Arbeitskreis Universitäre Erwachsenenbildung e. V.“ (AUE) 1970 als Dachverband der Weiterbildung an Hochschulen konstituiert. ${ }^{7}$ In der Vereinssatzung von 1971 heißt es programmatisch: Der Zweck des Vereins ist „die überregionale Förderung, Koordinierung und Repräsentation der von den Hochschulen getragenen Weiterbildung. Dazu gehört die Förderung der Forschung und Lehre auf dem Gebiet der Erwachsenenbildung“ ( $\sqrt{2}$, Abs.1). Eindeutig sind hier die Bindung und der enge Schulterschluss der universitären Weiterbildung, die später begrifflich in wissenschaftliche Weiterbildung umfirmiert wurde und auch die Fachhochschulen einbezog, an die akademische Disziplin der Erwachsenenbildung. In der Ursprungssatzung wird zugleich auch die Koppelung an die allgemeine Erwachsenen-/ Weiterbildung Rechnung getragen. So sieht die Satzung für den Fall der Auflösung des AUE-Vereins in $\ 13$ (Abs. 2) vor: „Das nach durchgeführter Auflösung vorhandene Vermögen fällt dem Deutschen Volkshochschulverband, Bonn“ zu. Diese „Vermögensbindung gilt auch bei Aufhebung des Vereins oder Wegfall seines bisherigen Zwecks“ (『2, Abs. 3, Satz 3).

Das Fernstudium repräsentiert im Unterschied zur wissenschaftlichen Weiterbildung, die im Konzert hochschulischer Bildungsangebote sektoral verankert ist und gelegentlich auch als eine Säule im Ausbildungsspektrum gesehen wird, nur ein Bildungsformat (Peters 1997), das völlig unabhängig von allen Sektoren oder Säulen zu betrachten ist. Das Kennzeichen dieses Formates ist, dass die konstitutive Wissenskommunikation, auf der Bildungsprozesse beruhen, auf Medien umgestellt wird. Damit wird eine Zeit- und Ortsunabhängigkeit des Lehrens und Lernens erreicht und es lassen sich Skaleneffekte realisieren, die der Bildung als eine wissensbasierte Dienstleistung normalerweise fremd sind. Im Unterschied zum Fernstudium geht das tradierte Bildungskonzept von einer Art von Versammlungspädagogik aus, die die raum/zeitliche Koexistenz der am Bildungsprozess beteiligten Akteure für nicht dispensierbar hält. Die Folge davon ist, dass Bildung eine überaus personalintensive Dienstleistung ist $^{8}$ und sich scheinbar allen Rationalisierungsbemühungen schon im Keim widersetzt. ${ }^{9}$

Mit dem Fernstudium ist grundsätzlich kein Präjudiz für die Weiterbildung verbunden. Der AUE war daher für die AG-F auch nicht so etwas wie der natürliche Hafen,

7 An der Wiege des AUE stand das in den 60er-Jahren praktizierte Konzept der Göttinger Seminarkurse, in die die Erfahrungen der britischen Besatzungsmacht zur Reeduaction eingegangen waren.

8 Das wird leicht daran erkennbar, dass grundsätzlich immer dann, wenn mehr Personen „beschult“ werden sollen, der Ruf nach einer Erhöhung der Zahl der Lehrkräfte laut wird. Die Formel lautet: Mehr Schüler, mehr Lehrer.

9 Skeptisch ließe sich allerdings fragen, ob nicht inzwischen die Apologeten der Selbstbildung hinter der Fassade des emphatisch beschworenen „Selbst“ bloß Rationalisierung betreiben, ohne das freilich so zu nennen. 
in den die Organisation als eine Art von Flaggschiff des Fernstudiums hätte einlaufen sollen. Das Fernstudium als Bildungsformat befasst sich originär nicht mit Fragen des Lehrens und Lernens Erwachsener, es geht ihm nicht um eine erwachsenengerechte Didaktik oder sonstige Problembestände, für die die Erwachsenenbildung ihre disziplinäre Zuständigkeit reklamiert. Konzediert werden kann jedoch, dass sich das Format des Fernstudiums in besonderer Weise für eine nebenberufliche Qualifizierung eignet und grundsätzlich eine Kombination von Studium und Beruf ermöglicht, die sich in Form von Präsenzangeboten eher mühsam umsetzen lässt. Ein nebenberuflich absolviertes Studium ist jedoch nicht zwingend ein Weiterbildungsstudium - und ein Weiterbildungsstudium nicht zwingend ein Fernstudium.

\section{Rahmungen}

Die für das Fernstudium konstitutive Mediatisierung der Wissenskommunikation führt zum Teil zu theoretischen Überhöhungen und überschießenden Interpretationen. Auch das ist eine Besonderheit, die das Fernstudium von anderen Bildungsformaten unterscheidet und vermutlich dem besonderen Charakter des Lehr-/Lernformates geschuldet ist.

Die Überinterpretation des Fernstudiums besteht beispielsweise in der Behauptung, dass es sich bei diesem Bildungsformat um die Realisierung eines ,autonomen Lernens“ handeln soll, das gleichsam die Inkarnation eines „selbstorganisierten bzw. selbstgesteuerten Lernens“ ist. Solche Charakterisierungen offenbaren indessen eher das ungläubige Staunen darüber, dass die Wissenskommunikation von der Mündlichkeit, d.h. einem anwesenden Lehrenden und seinem Rezipienten abgelöst und erfolgreich mediatisiert werden kann. Dieses Staunen bezeugt aber auch, dass die Charakterisierung des Fernstudiums sich letztlich nicht von der Folie der Präsenzlehrtradition befreit hat, sondern ihr tief verhaftet bleibt. Anders sind jedenfalls die um die Selbststeuerungsthematik rankenden Formulierungen kaum nachvollziehbar. Es soll demgegenüber hier die These vertreten werden, dass das Fernstudium weder selbst- noch fremdgesteuert ist. Es ist bestenfalls ein Lernen ohne unmittelbare soziale Kontrolle, da auch dann gelernt wird, wenn ein Banknachbar oder Lehrender nicht in der Nähe ist. Die in der mündlich praktizierten Wissenskommunikation mitlaufende Instruktion wird beim Fernstudium lediglich auf Medien umgestellt. Sie verschwindet dadurch nicht, wie das Narrativ des „Selbstlernes“ gern glauben machen möchte.

Dass die Gründungsmitglieder sich für das AUE-AG-Modell entschieden haben, beruhte letztlich auf pragmatischen Überlegungen. In erster Linie bestand ein Einvernehmen darin, dass man auf den Aufbau einer eigenen Organisation verzichten wollte. Um den Schulterschluss mit dem AUE praktizieren zu können, wurde in der Satzung der AG-F ein Bekenntnis zur wissenschaftlichen Weiterbildung abgelegt und das Fernstudium in das Profil des AUE eingepasst. Im Abschnitt „Zielsetzung“ der AG-F heißt es: „Die Arbeitsgemeinschaft für das Fernstudium an Hochschulen 
(AG-F) hat Studium und Lernen in der Verbindung mit Medien vornehmlich auf dem Gebiet der (berufsbezogenen) wissenschaftlichen Weiterbildung und der postgradualen Studien, soweit Hochschulen der Bundesrepublik Deutschland daran beteiligt sind, zum Gegenstand ihrer Tätigkeit. Die Mitglieder der AG-F verfolgen durch den Zusammenschluss insbesondere die Ziele,

- eine Plattform zu bilden für die Diskussion aller theoretischen und praktischen Dimensionen des Fernstudiums und des offenen Lernens an Hochschulen,

- das allgemeine Verständnis für mediales Lernen im Bereich (berufsbezogener) wissenschafticher Weiterbildung zu fördern,

- eine Infrastruktur aufzubauen für die überregionale Entwicklung und Verbreitung von Angeboten des (weiterbildenden) Fernstudiums an Hochschulen,

- das Fernstudium ihrer Hochschulen auf europäischer Ebene gemeinsam zu vertreten und sich an übernationalen Projekten auf dem Gebiet des Open and Distance Learning (ODL) zu beteiligen".

Wie die übrigen Sektionen auch, wird die AG-F von einem Sprecherrat geführt, der aus mehreren Personen besteht. Die Amtszeit beträgt jeweils zwei Jahre. Auf der ersten ordentlichen Mitgliederversammlung wurden Hans-Henning Kappel (Frankfurt/Main) zum Vorsitzenden des Sprecherrates und Helmut Vogt (Hamburg) und Ulrich Bernath (Oldenburg) als weitere Sprecher gewählt.

In der Namensnennung des AUE schlug sich die Aufnahme des Fernstudiums zunächst nicht nieder. Dies geschah erst mit der Änderung der Satzung im Jahr 2003 auf einer Mitgliederversammlung in Dresden. Aus dem ehemaligen AUE wurde die „Deutsche Gesellschaft für wissenschaftliche Weiterbildung und Fernstudium“. Seither ist das Fernstudium innerhalb der Standesorganisation der Weiterbildenden an Hochschulen prominent vertreten.

\section{Weitere (parallele) Entwicklungen}

Die erfolgreiche Konstitution der AG-F als Interessenvertretung von Fernlehranbietern kann nicht für sich in Anspruch nehmen, die erste und einzige Organisation dieser Art zu sein. Das Erstgeburtsrecht gebührt vielmehr dem „Arbeitskreis korrektes Fernlehrwesen“ (AkF), der 1969 aus der Taufe gehoben worden war. Übergegangen ist dieser Arbeitskreis 1980 in den "Deutschen Fernschulverband“ (DFV), der 2003 schließlich in das „Forum DistancE-Learning“ mündete. Das Forum kann als die Schwester- oder Bruderorganisation der AG-F aufseiten der privaten Bildungsanbieter betrachtet werden, da sich im Forum hauptsächlich privatwirtschaftliche Anbieter versammelt haben, während in der AG-F vornehmlich staatliche Fernstudienanbieter zu finden sind. Das ist nicht der einzige Unterschied zwischen den beiden Organisationen: So sind im Forum nicht nur akademische Einrichtungen, sondern auch nicht-akademische Institutionen des Fernlehrmarktes vertreten, was den kooperativen Beziehungen beider Institutionen keinen Abbruch tut. Zwischen den beiden Fernstudien- oder Fernlehrorganisationen besteht seit Jahren ein kollegiales 
Verhältnis. Eine Konkurrenz oder einen Wettbewerb gibt es nicht. In einer Protokollnotiz des AUE findet sich die Bemerkung, dass die AG-F die „aktivste Sektion“ innerhalb des Verbandes ist. Dieses Kompliment adressiert eine Vielzahl von Aktivitäten, die die AG-F in der Vergangenheit unternommen hat. Für die überaus ehrgeizigen Ambitionen der Organisation spricht, dass sie für einige Jahre Mitglied beim "International Council for Open and Distance Education" (ICDE), dem Weltverband des Fernstudiums wurde. Der Impuls zur Mitgliedschaft im Weltverband ging wesentlich von einer „German-Group-Tour“ aus. Im Jahr 1997 machten sich zahlreiche Mitglieder der AG-F auf den Weg zur 18th ICDE-World Conference an der „Pennsylvania State-University“ in den USA. Die Gruppenreise diente zugleich dazu, verschiedene Fernstudienanbieter und deren Ansätze in den USA kennenzulernen. Die Reise zur Weltkonferenz wurde so zu einer Bildungsreise. Sie umfasste die Stationen: SUNY New York - Empire State College in Saratoga Springs, Pennsylvania State-University und die University of Maryland - University College. Die Reise bot den Teilnehmenden nicht nur interessante Einblicke in das amerikanische Fernlehrewesen, sondern stärkte zugleich das Wir-Gefühl der Reisenden. Zu den internationalen Auftritten der AG-F-Mitglieder gehörte schließlich im Jahr 2000 die Beteiligung am ersten „World Education Market“ in Vancouver, an dem sich mehr als 450 Unternehmen aus 34 Ländern beteiligt hatten. Eine Anknüpfung an die in den USA gesammelten Erfahrungen bot den AG-F-Mitgliedern 2001 schließlich die Teilnahme an der „ICDE-Weltkonferenz“ in Düsseldorf. Die AG-F präsentierte sich dort in einem eigenen Track zum Thema: „Fernlehre und Fernstudium im deutschsprachigen Raum“.

\section{Das Projekt „Psychologische Gesundheitsförderung für Pflegepersonal“}

Die Aktivitäten beschränkten sich allerdings nicht auf die Partizipation an den Veranstaltungen des Weltverbandes des Fernstudiums. Die Mitglieder bildeten untereinander ein Netzwerk zur gemeinschaftlichen Durchführung fernstudiengestützter Bildungsmaßnahmen auf der Ebene von Zertifikatslehrgängen. Gebildet wurde ein Netzwerk gleichberechtigter Partner. Das Durchführungskonzept und das Material wurden geteilt, jeder an seinem Standort. Das war bei „GruZi“ so. Hierbei handelt es sich um ein im Verbund durchgeführtes Projekt, das sich gezielt an Grundwehrdienst- und Zivildienstleistende wandte und diesem Klientel studienvorbereitende und -orientierende Programme zur Mathematik anbot. $\mathrm{Zu}$ einem herausragenden Verbundprojekt entwickelte sich das Programm der „Psychologischen Gesundheitsförderung für Pflegepersonal“, das in Oldenburg entwickelt worden war. In einer Selbstbeschreibung des Projekts heißt es dazu: „Zum Beispiel bilden die Fernlehreinheiten des Oldenburger Entwicklungsteams der ,Psychologischen Gesundheitsförderung die Grundlagen für eigenverantwortliche Studienprogramme an anderen, im Prinzip beliebig vielen Hochschulstandorten. Die Fernlehreinheiten sind als 
Module für ein denkbares nebenberufliches Studium für Pflegekräfte entwickelt worden. Sie werden von Universitäten in Berlin, Bern, Hamburg, Hildesheim, Frankfurt, Karlsruhe, Kaiserslautern und Koblenz-Landau in halb- bzw. einjährigen Weiterbildungsprogrammen eingesetzt und erreichten bereits über 600 Teilnehmende. Das Kuratorium für Dialyse und Nierentransplantation e. V. in Neu-Isenburg hat die Zusammenarbeit mit dem Fernstudienzentrum der Universität Oldenburg und dem bestehenden Netzwerk gesucht, um eine weiterentwickelte Version der ,Psychologischen Gesundheitsförderung' mehr als 1.000 seiner Pflegekräfte als ein Fernstudienprojekt zur Weiterbildung anzubieten“ (Bernath/Kleinschmidt 1995). Es folgte über viele Jahre die aktive Beteiligung an der „LearnTec“ in Karlsruhe in Form der Ausrichtung eines ganztägigen Spezialkongresses.

\section{Die digitale Wende}

Die Beteiligung an der thematisch am Einsatz und der Verwendung von „Bildungstechnologien“ orientierten Messe war Ausdruck und Reaktion auf eine veränderte Situation im Bereich des Fernstudiums bzw. der Fernlehre. Bis zum Anfang der goer-Jahre des vergangenen Jahrhunderts herrschte das klassische, papierbasierte Fernstudium vor. Das Fernstudium war gewissermaßen bis dahin das legitime Produkt der Gutenberggalaxis. Mit dem Aufkommen von „Multimedia“, d.h. der Möglichkeit unterschiedliche Medien und Medienformate auf digitaler Basis zusammenzubinden und verfügbar zu machen, wurde das Zeitalter des netzbasierten Lehrens und Lernens eingeläutet. Das Zeitalter der Gutenberggalaxis machte den Weg für die Internetgalaxis frei. In dieser Umbruchsituation zeigte die AG-F auf der „Technologiemesse“ Flagge und inskribierte sich gleichsam in die neue Entwicklung. Zum Bannerträger reichte das Engagement freilich nicht. Obwohl das Fernstudium seit seinen Anfängen technologiebasiertes Lehren und Lernen ist und daher über eine Kernkompetenz im Bereich des Einsatzes von Bildungstechnologien verfügt, bildete sich eine „E-Community“ jenseits des Fernstudiums und seiner Akteure aus, die sich um die „Gesellschaft für Medien in der Wissenschaft“ (GMW) versammelte.

\section{Qualitätssicherung}

Die aufkommende und anhaltende Konjunktur der Qualitätsthematik, die eine neue Form der Regierung im Sinne Foucaults darstellt, forderte die AG-F zu einer proaktiven Haltung heraus. Es wurden sogenannte „gute Regeln“ für die Fernlehre erarbeitet. Sie haben bis heute Bestand und sind der Erkenntnis geschuldet, dass das distanzüberwindende Lehren und Lernen mithilfe von Bildungstechnologien Eigenheiten aufweist, die an die Qualitätssicherung besondere Anforderungen stellen.

Die Gründergeneration der AG-F ist inzwischen Bestandteil eines kommunikativen Gedächtnisses. Die überwiegende Zahl derer, die damals Pate gestanden haben, sind 
mittlerweile aus dem aktiven Berufsleben ausgeschieden. Gegenwärtig zählt die AGF 63 Mitglieder. Zum Ehrenmitglied der Organisation wurde Börje Holmberg ernannt, der langjährige Direktor des „Instituts für Fernstudienforschung“ an der FernUniversität in Hagen und Gründungspräsident der „Wilhelm Büchner Hochschule“ in Darmstadt war. Er ist einer der führenden internationalen Fernstudienexperten neben Otto Peters, dem Gründungsrektor der FernUniversität u. a.

\section{Strukturwandel}

Das Fernstudium ist keineswegs aus einer Nische hervorgegangen, wie manche vermeintliche Experten meinen. Im Gegenteil blickt es auf eine 150-jährige Geschichte zurück und ist dabei vielerorts vor allem international breit angelegt gewesen. In den letzten 25 Jahren hat sich sein Gesicht jedoch strukturell fundamental verändert. Es ist inzwischen weitgehend digitalisiert worden und hat sich zu Online-Distance-Education weiterentwickelt. Von vielen Institutionen des Fernstudiums wird die neue Form der Lehre und des Lernens in globalem Maßstab angeboten. Internationale Experten glauben, dass die Verbindung von Fernstudium und Online Learning sich in Zukunft zum Mainstream der wissenschaftlichen Lehre entwickeln könnte. Heute ist das Fernstudium daher längst keine Erscheinung am Rande mehr. Seine rasante Entwicklung während der letzten 25 Jahre hat ein generelles Umdenken erforderlich gemacht.

\section{Literatur}

Bernath, U./Kleinschmidt, A. (1995): Netzwerke. Ein neuer Weg im Fernstudium. In: Pressestelle der Carl von Ossietzky Universität Oldenburg (Hrsg.): UNI-INFO 22. Jg. 4, S. 5. http://www.c3l.uni-oldenburg.de/literat/netzwerk.htm. [Zugriff: 19.08.2015]

Peters, O. (1997): Didaktik des Fernstudiums. München.

Pfundtner, R. (1995): Fernstudium an Fachhochschulen. Das Modell der Fernstudienbrückenkurse in den neuen Bundeländern. In: Beiträge zur Hochschulforschung, 4, S. 379-404.

Schwier, C.G. (1962): Fernstudium als neuer Bildungsweg. In: Friedrich Naumann Stiftung (Hrsg.): Gegenwartsaufgabe der Erwachsenenbildung. Festschrift zum 70. Geburtstag von Richard Freudenberg. Köln: Opladen. S. 38 ff. 



\title{
Bundesarbeitsgemeinschaft Wissenschaftliche Weiterbildung für Ältere (BAG WiWA)
}

Thomas Bertram/Silvia Dabo-Cruz/Karin Pauls/

Michael Vesper

\begin{abstract}
Die Bundesarbeitsgemeinschaft Wissenschaftliche Weiterbildung für Ältere (BAG WiWA) ist eine Arbeitsgemeinschaft der DGWF. Der Artikel beschreibt ihre Entstehungsgeschichte und die Entwicklungsschritte beim Zusammenschluss mit der DGWF und ihrer Vorläuferorganisation. Neben der verbandspolitischen Entwicklung der BAG WiWA erfolgt die Verortung im Kontext des Bologna-Prozesses und die aktuelle Positionierung der BAG WiWA im Feld der Bildung für ältere Erwachsene. Dabei liegt ein Schwerpunkt auf neuen Zielgruppenanalysen und Forschungsfragen, mit denen zukünftig belastbare empirische Daten zur Bildung Älterer erhoben werden können. Abschließend werden die künftigen Herausforderungen für die BAG WiWA skizziert.
\end{abstract}

\section{Einleitung}

Die Bundesarbeitsgemeinschaft Wissenschaftliche Weiterbildung für Ältere (BAG WiWA) ist eine der vier Arbeitsgemeinschaften der DGWF. Sie setzt sich zusammen aus Einrichtungen, die an Hochschulen oder in enger Kooperation mit Hochschulen für die Entwicklung und Organisation wissenschaftlicher Weiterbildungsangebote für ältere Erwachsene zuständig sind. Sie organisiert den institutionellen Austausch innerhalb der Mitgliedsorganisationen wie auch zu externen Kooperationspartnern und fördert die bundesweite Weiterentwicklung der Weiterbildung Älterer an Hochschulen. Hauptinstrumente sind dabei die Jahrestagungen an wechselnden Orten sowie der Sprecher/innen/rat als gewählte Vertretung. Derzeit gehören der BAG WiWA 60 Einrichtungen an sowie zehn persönliche Mitglieder. ${ }^{1}$

1 Einen Überblick über die Mitgliedsorganisationen bietet die Homepage der BAG WiWA www.bagwiwa.de. 
Im Folgenden soll zunächst die Entstehung und Entwicklung der BAG WiWA kurz beschrieben werden. Daran anschließend werden zwei aktuelle Schwerpunkte ihrer Arbeit dargestellt, die Frage der Positionierung durch interne und externe Kooperation sowie die Erforschung ihrer Zielgruppen.

\section{Entstehung}

Seit 1994 ist die BAG WiWA als Arbeitsgemeinschaft in die DGWF (zu diesem Zeitpunkt noch Arbeitskreis Universitäre Erwachsenenbildung e. V. - AUE) eingebunden. Ihre Geschichte beginnt allerdings schon einige Jahre bevor sie Teil der DGWF wurde und ist in ihren Anfängen eng mit der Bewegung zur Öffnung der Hochschulen für ältere Erwachsene verbunden.

Im Dezember 1979 fand an der Universität Oldenburg der erste internationale Workshop „Öffnung der Universitäten für ältere Erwachsene“ statt, veranstaltet vom Zentrum für wissenschaftliche Weiterbildung (ZWW) der Universität Oldenburg in Kooperation mit der Interdisziplinären Arbeitsgruppe für Angewandte Soziale Gerontologie (ASG) der Gesamthochschule Kassel, maßgeblich von Barbara Fülgraff und Brunhilde Arnold konzipiert. Dabei wurde - in Deutschland in dieser überregionalen Form zum ersten Mal - die Entwicklung von Universitäten des 3. Lebensalters in Frankreich und anderen europäischen Ländern (vgl. Swindell/Thompson 1995; Zahn 1993) aufgegriffen und mit den Anliegen der damaligen Bildungsoffensive verbunden, in der Absicht, wissenschaftliche Bildungsangebote an Hochschulen für ältere Menschen konzeptionell und organisatorisch auf den Weg zu bringen. Damit fand auch eine Akzentuierung gerontologischer Forschungsperspektiven Eingang in den Kontext der wissenschaftlichen Weiterbildung (Zentrum für wissenschaftliche Weiterbildung Oldenburg 1980).

Der Workshop hatte eine nachhaltige Wirkung. An verschiedenen Hochschulen wurden Studienangebote für Ältere entwickelt, teilweise auch in Modellversuchen, z. B. an den Universitäten Dortmund und Marburg. Parallel dazu begannen sich die Institutionen und verantwortlichen Personen zu vernetzen. Fünf weitere Internationale Workshops „Öffnung der Hochschulen für ältere Erwachsene“ wurden veranstaltet, 1981 und 1984 in Dortmund, 1987 in Marburg, 1990 in Frankfurt a. M. und 1996 in Münster. Dem wachsenden Bedürfnis nach einem intensiveren fachlichen und kollegialen Austausch wurde mit der Gründung einer Bundesarbeitsgemeinschaft „Öffnung der Hochschulen für ältere Erwachsene“ (BAG) Rechnung getragen. An der konstituierenden Sitzung am 15.11.1985 an der Universität Dortmund unter Federführung von Ludger Veelken nahmen 44 Vertreterinnen und Vertreter von 27 Institutionen teil. ${ }^{2}$

vgl. Sitzungsprotokoll, Dortmund 19.12.1985, unveröffentlicht. 


\section{Erste Entwicklungsschritte und Zusammenschluss mit der AUE (heute DGWF)}

Die BAG hielt zwei Plenumstreffen pro Jahr ab, ein Sprecher/innen/rat wurde eingesetzt und eine Geschäftsstelle eingerichtet. Außerdem wurde das „BAG-Info“ herausgegeben, das über wichtige Ereignisse in den Mitgliedsorganisationen, Forschungen und Literatur zum Themenbereich sowie interessante Tagungen informierte. ${ }^{3}$ Gemeinsame Aufgaben waren zunächst die „Absicherung und Verstetigung der Angebote“, in der Folge die „Ausdifferenzierung der Modelle“ und - nachdem die Aufbruchsphase abgeschlossen war - die „Institutionalisierung in der Vielfalt“ (Sagebiel 2006b, S.192).

Die Vielfalt der Organisationsformen und Angebote bleibt bis heute ein Kennzeichen der wissenschaftlichen Weiterbildung für Ältere. Im Kontext von unterschiedlichen Vorstellungen, Bedingungen und Ressourcen an den beteiligten Hochschulen haben sich verschiedene Angebotsmodelle herausgebildet. Am häufigsten ist das Gasthörermodell vertreten, bestehend aus geöffneten Veranstaltungen des RegelStudienangebots einer Hochschule. Diese werden an vielen Standorten durch gesonderte Veranstaltungen in unterschiedlichem Umfang sowie durch Zertifikatsstudiengänge ergänzt. An einigen wenigen Einrichtungen bilden spezielle Angebote für ältere Studierende den größten Teil des Angebots. Bei aller Differenzierung verfolgen die in der BAG WiWA organisierten Einrichtungen als gemeinsame zentrale Ziele die Integration des Weiterbildungsangebots für Ältere in das Studienangebot der Hochschulen sowie einen intergenerationellen Ansatz.

Nach 1989 kamen einige ostdeutsche Institutionen, teils Neugründungen, teils erneuerte Einrichtungen auf der Basis der bereits vor 1989 bestehenden Veteranenoder Seniorenkollegs, zur BAG hinzu. Sie wuchs Anfang der 1990er-Jahre auf fast 50 Mitglieder an. Während die Arbeit der BAG von großem Engagement geprägt war, verliefen die Bemühungen um eine Verstetigung der Bildungsangebote für Ältere nicht immer erfolgreich. ${ }^{4}$ Mit einer 1991 verabschiedeten Resolution bezieht die BAG kritisch Stellung zur teilweise prekären Lage der wissenschaftlichen Weiterbildung an den Hochschulen. ${ }^{5}$

Auch wenn es der BAG gelungen war, ihre Arbeit als informelles Netzwerk zu verstetigen, so stellte sich nach knapp zehnjährigem Bestehen angesichts der genannten Probleme die Frage nach einer Organisationsform, die eine größere hochschulund bildungspolitische Einflussnahme ermöglichte. Da in der Mitgliedschaft der BAG zu anderen Bereichen der wissenschaftlichen Weiterbildung sowie zum Arbeitskreis Universitäre Erwachsenenbildung (AUE) gute Kontakte und teilweise per-

3 vgl. BAG-Info Nr. 1, Dortmund 1986, bis Nr. 13, Münster 1999.

4 Beispielsweise wurde das im Marburger Modellversuch „Entwicklung nicht berufsqualifizierender Studiengänge für ältere Erwachsene (1985-1988)“ entwickelte Programm nach Abschluss des Versuchs nicht fortgeführt.

5 vgl. Resolution zur Lage der Wissenschaftlichen Weiterbildung für Ältere Erwachsene in der BRD, von der BAG verabschiedet am 8.3.1991 in Schwäbisch Gmünd. 
sonelle und inhaltliche Überschneidungen bestanden, erschien es naheliegend, eine engere Verbindung zum AUE zu suchen. 1994 schloss sich die BAG, mit neuer Bezeichnung „Bundesarbeitsgemeinschaft wissenschaftliche Weiterbildung für Ältere“ (BAG WiWA), dem AUE an. Dieser Schritt war von einer Diskussion um das Selbstverständnis begleitet und führte zu einer Klärung und Formalisierung von Aufgaben und Rollen innerhalb der Arbeitsgruppenstruktur. Die Mitgliedschaft in der BAG WiWA war nun an eine Mitgliedschaft im AUE gekoppelt, ein/e Vertreter/in wurde kooptiertes Mitglied des AUE-Vorstandes. Verschiedene Arbeitsbereiche, z. B. Information, Publikationen und Tagungsplanungen, wurden in der Folge in den Kontext des AUE integriert.

Wie weit es auf diese Weise gelang und bis heute gelingt, der wissenschaftlichen Weiterbildung für Ältere mehr Bedeutung zu verschaffen und die Interessen der BAG WiWA-Mitglieder wirksamer zu vertreten, kann als ein dynamischer Prozess beschrieben werden. So sehr in bestimmten Situationen konstruktive gemeinsame Strategien und Aktionen hilfreich sind, so bilden doch ältere Studierende eine Zielgruppe, deren Anliegen nicht in der berufsbezogenen Weiterbildung aufgehen, die den primären Fokus der DGWF bildet. Themen des Studiums im Alter sind daher im Diskurs der DGWF zeitweise unterrepräsentiert. Die Präsentations- und Publikationsmöglichkeiten, die die Jahrestagungen und Veröffentlichungen der DGWF bieten, können auch deshalb von BAG WiWA-Mitgliedern nicht kontinuierlich ausgeschöpft werden, weil im Bereich des Studiums im Alter Ressourcen für Forschung und Entwicklung knapp bemessen sind. Aktuell bildet die Intensivierung von Forschungsaktivitäten einen Arbeitsschwerpunkt der BAG WiWA (siehe Abschnitt 5).

Trotz der inzwischen unstrittigen Bedeutung von Bildung für alle Altersgruppen im demografischen und strukturellen Wandel bleiben eine breite Akzeptanz der Bildung im Alter und eine angemessene Forschungsförderung aus. Die Oldenburger Erklärung, verabschiedet auf der Jahrestagung 2013 in Oldenburg, thematisiert dieses Spannungsfeld (siehe Abschnitt 4).

Neben solchen gesellschafts- und bildungspolitisch akzentuierten Stellungnahmen greift die BAG WiWA in ihren Jahrestagungen und den Publikationen aus ihren Reihen Themen auf, die sich unmittelbar mit dem Angebot und den Lehr-/Lernformen auseinandersetzen, wie Intergenerationelles Lernen (vgl. u.a. Keil/Brunner 1998), Forschendes Lernen (vgl. u. a. Meyer-Wolters et al. 2012), Neue Medien (vgl. u. a. Stadelhofer 1998). Netzwerkbildung und europäische Zusammenarbeit (vgl. u. a. Stadelhofer 2009) sind weitere wiederkehrende Themen. Auch der kollegiale Austausch von Good Practice im organisationalen Bereich hat traditionell einen hohen Stellenwert. Über das unmittelbare eigene Feld hinaus weisen Publikationen zu grundlegenden Bildungsfragen des Alters, die im Kontext der BAG WiWA initiiert und herausgegeben wurden (vgl. u.a. Becker/Veelken/Wallraven 2006; Malwitz-Schütte 2000). 


\section{Entwicklung im Kontext des Bologna-Prozesses}

Mit Bezug auf die Implementierung der Bologna-Beschlüsse an den Hochschulen konstatiert Felizitas Sagebiel 2006 eine Bedrohung der wissenschaftlichen Weiterbildung Älterer (Sagebiel 2006a). Das modularisierte Studienangebot sei weniger attraktiv für Seniorstudierende, die strikte Ausrichtung auf schnelle Berufsqualifizierung böte weniger Anlass und Raum für den Dialog zwischen jungen und älteren Studierenden. „Besonders für Studienmodelle [...], die strukturell von der Öffnung von Lehrveranstaltungen abhängig sind, verschlechtert sich die Situation“ (ebd. S. 12) bis hin zu einer Ausgliederung von Studienangeboten für Ältere und damit der Preisgabe der Integrationsidee.

Auf ihrer Jahrestagung 2006 in Frankfurt hat sich die BAG WiWA unter der Themenstellung „Demographischer Wandel und Bolognaprozess. Auswirkungen auf das Seniorenstudium“ mit dieser veränderten Situation an den Hochschulen kritisch auseinandergesetzt. Nicht von ungefähr wurde die Universität Frankfurt für diese Tagung ausgewählt. Dort hatte die Goethe-Universität den Zugang von Studierenden der Universität des 3. Lebensalters (zur U3 L siehe Dabo-Cruz 2015) zu regulären Lehrveranstaltungen drastisch eingeschränkt (Dabo-Cruz 2006). Probleme des zunehmenden Effizienzdrucks und der Ökonomisierung der Studienangebote in der Weiterbildung, auch Folgen der Bologna-Strategie, wurden auf der Hamburger BAGWiWA-Jahrestagung 2008 wieder aufgegriffen und in ihren Konsequenzen für die Entwicklung von neuen Angebotsformen und qualitätssichernden Maßnahmen diskutiert.

Die Tatsache, dass im Kontext der Implementierung des Lebenslangen Lernens an Hochschulen Seniorstudierende zunehmend als relevante Studierendengruppe adressiert werden ${ }^{6}$, eröffnet heute weiterführende Perspektiven. Eine Zukunftsaufgabe ist daher die Entwicklung des Studiums für Ältere zu einem „integralen Bestandteil der Angebotspalette einer Hochschule“. ${ }^{7}$

\section{Positionierung}

Anlässlich ihrer Oldenburger Jahrestagung im Februar 2013 präzisierte die BAG WiWA ihre Ziele und formulierte eine öffentliche Erklärung. In dieser Oldenburger Erklärung $^{8}$ stellt die BAG WiWA in fünf Punkten Forderungen für eine zukunftsweisende Entwicklung auf. Die BAG WiWA hatte sich in Oldenburg u. a. mit den Folgen des demografischen Wandels in seinen Konsequenzen für Bildung und Studium

6 vgl. „European Universities' Charter on Lifelong Learning“, zitiert nach Vogt, H. (2015): Studium für Ältere im Kontext lebenslangen Lernens an Hochschulen, Vortrag anlässlich der BAG WiWA-Tagung 2015 am 04. März 2015 in Kiel. https://www.awb.uni-kiel.de/de/allgemeine-bildungsangebote/bildungsangebote-der-bag-wiwa-hochschulen/dateien/ impulsvortrag-helmut-vogt [Zugriff:10.11.2016].

7 ebenda.

8 Oldenburger Erklärung https://dgwf.net/fileadmin/user_upload/DGWF-BAG-WiWA-Oldenburger-Erklaerung.pdf [Zugriff: 25.02.2016] 
im Alter befasst. „Es ist jetzt schon zu beobachten, dass mit der Veränderung des Altersaufbaus der Bevölkerung auch der Anteil aktiver und bildungsinteressierter älterer Menschen wächst. Daher ist eine Weiterentwicklung von Strukturen notwendig, die Bildung und Lernen in allen, besonders auch in späten Lebensphasen, ermöglichen und fördern. “9 Mit der Oldenburger Erklärung hat die BAG WIWA sich öffentlich neu positioniert und ihre Ziele und zukünftige Aufgabenfelder benannt. Diese beinhalten sowohl Hochschulfragen wie die Berücksichtigung wissenschaftlicher Weiterbildung bei Lehrdeputaten und der Kapazitätsplanung wie auch Fragen nach Zielgruppen, Inhalten und neuen Formaten und Produkten.

Wie kann nun ein stärkeres Bewusstsein für Bildungsfragen erreicht werden? Dies kann zum einen über Einflussnahme auf politische Erklärungen (vgl. Frankfurter Erklärung der BAGSO aus Anlass des 11. Deutschen Seniorentages), Mitwirkung bei Studien (z. B. vom Sprecher/innen/rat erstellter Fragenkatalog zum Generali-Zukunftsfonds im Jahr 2014) und ein verstärktes Engagement in landes- und bundespolitischen Arbeitsgruppen sowie durch internationale Kontakte erfolgen. Die BAG WiWA organisiert diesen institutionellen Austausch. Als langjährige externe Partner im Themenfeld Alter, Altern und Bildung im Alter, insbesondere Weiterbildung an Hochschulen, sind besonders drei Organisationen mit unterschiedlichen Schwerpunkten in diesen Bildungsbereichen zu nennen:

- AIUTA (Association Internationale des Universités du Troisième Age),

- EFOS (European Federation of Older Students),

- DENISS (Deutsches Netzwerk der Interessenvertretungen von Senior-Studierenden).

Im Zeitverlauf ist die Intensität des Austauschs zu externen Organisationen nicht immer gleichbleibend, da die Kontaktpflege auch vom persönlichen Engagement einzelner Mitglieder abhängt.

Mit dem Beitritt der DGWF zur Bundesarbeitsgemeinschaft der Senioren-Organisationen (BAGSO) entstand ein neues Netz mit Partnern, deren Sicht und Arbeitsschwerpunkte vor allem im karitativen Bereich und weniger auf Bildung für Ältere ausgerichtet sind. Die BAGSO ist für die BAG WiWA dennoch ein interessanter Partner, der überaus aktiv und erfolgreich Altersthemen in die Politik und Gesellschaft hineinträgt. Die Deutschen Seniorentage, die BAGSO-Nachrichten und ein Newsletter bieten der BAG WiWA Präsentations- und Publikationsmöglichkeiten, der wachsende Einfluss der BAGSO auf politische Entscheidungsträger wird mittelund längerfristig auch der BAG WiWA nutzen, ihre Anliegen stärker einzubringen.

Aber auch innovative Formate und neue Produkte „zwischen Bildungsauftrag und Markt ${ }^{\text {"10 }}$ können hier wesentliche Entwicklungsschritte leisten. Die BAG WiWA wird hierbei zur Koordinatorin oder auch selbst zur Anbieterin von wissenschaftlicher

9 ebenda.

10 Jahrestagung 2015 in Kiel; siehe: www.awb.uni-kiel.de/de/allgemeine-bildungsangebote/bildungsangebote-der-bagwiwa-hochschulen [Zugriff: 16.01.2016]. 
Weiterbildung für Ältere. Bestehende Projekte wie die bundesweite Online-Ringvorlesung ${ }^{11}$, erste kooperative Bildungsangebote mehrerer Einrichtungen (z. B. Sommer 2014 mit den Universitäten Trier, Hamburg und Kiel) und die geplante Entwicklung von Webinaren bis hin zu einem „Online Seniorenstudium“ weisen einen möglichen Weg.

Die institutionelle Verankerung der Bildung für Ältere in den Hochschulen kann insbesondere dann als ein Vorteil betrachtet werden, wenn es gelingt, wissenschaftliche Erkenntnisse und das gesellschaftliche und politische Gewicht der Hochschulen für die Diskussion um Bildung im Alter wie auch bei der Entwicklung neuer Weiterbildungsprodukte fruchtbar $\mathrm{zu}$ machen. Hochschulangebote, Wissenschaftlichkeit, überregionaler Verbund sind die entscheidenden Stichworte für die besondere Stellung am Markt. Die Vielzahl und große Vielfalt der Mitgliedsorganisationen stellen ein bisher für die Vermarktung gemeinsamer Produkte im Bildungsmarkt zu wenig genutztes Potenzial dar. Die Bereitstellung vernetzter Bildungsangebote durch die BAG WiWA und deren Präsentation auf den Webseiten sowohl der Mitgliedereinrichtungen wie auch der BAG WiWA könnte einen neuen Zugang zu der mittelbaren Zielgruppe der Teilnehmenden schaffen und insgesamt die öffentliche Wirkung aller Aktivitäten der BAG WiWA verstärken.

\section{Zielgruppenanalyse und Forschungsfragen}

Der Erforschung der Zielgruppen der BAG WiWA kann man sich von zwei Seiten nähern. Zum einen gibt es die unmittelbare Zielgruppe der BAG WiWA-Mitglieder; dies sind im Wesentlichen die Anbieter wissenschaftlicher Weiterbildung für Ältere. Zum anderen gibt es eine mittelbare Zielgruppe, nämlich die Teilnehmenden an wissenschaftlicher Weiterbildung für Ältere.

Empirische Untersuchungen über die unmittelbare Zielgruppe der Anbieter hat es zuletzt 2001 in einer bundesweiten Erhebung von Winfried Saup (2001) gegeben und daran anschließend 2009 eine Erhebung von Felizitas Sagebiel und Jennifer Dahmen (2009) zu ausgewählten Anbietern. Zurzeit gibt es in Deutschland insgesamt 88 staatliche Universitäten. ${ }^{12}$ An 45 Universitäten gibt es lediglich die Möglichkeit der Gasthörerschaft (d.h. nur Öffnung des regulären Lehrangebots ohne zusätzliche Beratungs- oder Veranstaltungsangebote). An 43 weiteren Hochschulen gibt es dagegen über die allgemeine Gasthörerschaft hinausgehende zusätzliche Angebote für Ältere. Daneben gibt es weitere private Einrichtungen, die entsprechende Programme anbieten; allerdings sind nicht alle staatlichen wie privaten Träger Mitglieder der BAG WiWA. Einen aktuellen Stand über Angebots- und Organisationsformen der Anbieterseite möglichst bundesweit zu erheben, kann als eine zukünftige Aufgabe der BAG WiWA identifiziert werden. Die Entwicklung der mittelbaren Ziel-

11 vgl. www.online-ringvorlesung.de [Zugriff: 16.01.2016].

12 nach Rathmann, Annika; unveröffentlichtes Manuskript, Magdeburg 2014. 
gruppe der Teilnehmenden ist im Laufe der Zeit mit zahlreichen Studien (meist in Form der klassischen Teilnehmerbefragung) recht gut begleitet worden. Die Anbieter, insbesondere die Mitglieder der BAG WiWA, sind hier die entscheidende Schnittstelle, denn nur sie erreichen die Teilnehmenden unmittelbar. Auch wenn es verschiedene nationale und internationale Studien zu dieser Zielgruppe gibt ${ }^{13}$, so sind für die BAG WiWA die lokalen Untersuchungen ihrer Mitglieder besonders interessant. Im Vergleich mit den übergreifenden Studien ermöglichen sie eine Validierung und Spezifizierung der Ergebnisse.

Es kommt nun darauf an, vorliegende Ergebnisse zu sammeln und möglichst allen Mitgliedern zugänglich zu machen ${ }^{14}$ sowie neue Untersuchungen mit möglichst einheitlichen und vergleichbaren Instrumenten durchzuführen. Beiden Zielen widmet sich ein Arbeitskreis der BAG WiWA, der sich auf der Jahrestagung 2015 in Kiel zusammengefunden hat und sich seitdem regelmäßig trifft. In dem so bezeichneten BAG-WiWA-Arbeitskreis Forschungsfragen und Statistik wurden in einem ersten Arbeitspaket Studien der letzten zehn Jahre aus sieben deutschen Städten ${ }^{15}$ in einer Synopse zusammengefasst, um daraus möglichst einheitliche und universell einsetzbare Items zu extrahieren. Als ein erstes Ergebnis dieser Arbeit wurde ein Fragebogen entwickelt, der im Frühjahr 2016 an den Universitäten Mannheim und Mainz in einem Pre-Test zum Einsatz kommt.

Was sagen bisherige Befunde über die mittelbare Zielgruppe der Teilnehmenden und was sind die Hypothesen für zukünftige Studien und Aufgabengebiete? In der hier gebotenen Kürze kann darauf nur exemplarisch geantwortet werden. Außer Frage dürfte die lebenslange Lernfähigkeit des Menschen stehen, die durch Studien der namhaftesten Alternsforscher inzwischen hinreichend belegt und publiziert worden ist. ${ }^{16}$ Die Fachdisziplinen der Psychologie und Soziologie, insbesondere des Alters, sowie der Gerontologie und Geragogik und in den letzten Jahren sicher auch der Neurowissenschaften waren und sind von hoher Bedeutung für die Arbeit der BAG WiWA, sollen aber hier nicht näher thematisiert werden. ${ }^{17}$

Für Angaben zu weitestgehend standortunabhängigen soziodemografischen Merkmalen von Teilnehmenden an Angeboten der wissenschaftlichen Weiterbildung für Ältere wurden hier die Studien der oben genannten Synopse zugrunde gelegt. Folgende Situation kann demnach beschrieben werden:

- Das Geschlechterverhältnis ist nahezu ausgeglichen, ein höherer Anteil von Frauen oder Männern variiert unter den Standorten nur geringfügig.

13 vgl. u. a.: nationale Studien: EdAge-Studie, Ludwig-Maximilians-Universität, München 2009; Generali Altersstudie. Zukunftsfonds der Generali Deutschland Holding AG, Köln 2013.; CiLL - Competencies in Later Life. Deutsches Institut für Erwachsenenbildung, Bonn 2014.

internationale Studien: PIAAC - Programme for the International Assessment of Adult Competencies. OECD, 2014.

14 Eine Bereitstellung der Studien auf der Internetseite der BAC WiWA ist in Vorbereitung.

15 Hannover, Köln, Leipzig, Magdeburg, Münster, Oldenburg, Stuttgart.

16 vgl. Publikationen von Paul B. Baltes, Ursula Lehr, Martin Korte, Andrea Kruse u. a.

17 Das Deutsche Zentrum für Altersfragen bietet auf seiner Internetseite hierzu eine umfangreiche Literaturdatenbank: http://www.dza.de/bibliothek/gerolit-der-online-katalog.html [Zugriff: 03.01.2016]. 
- Die Teilnehmenden kommen zum großen Teil aus bildungsaffinen Milieus, knapp zwei Drittel haben eine Hochschulzugangsberechtigung, rund die Hälfte von ihnen hat bereits einen akademischen Abschluss. Bildungsferne Milieus werden zwar auch erreicht, sind an allen Standorten aber deutlich unterrepräsentiert (Tippelt 2009).

- Das Durchschnittsalter liegt nach den sieben zugrunde gelegten Studien bei 67 Jahren.

- Fragt man nach der Motivation für die Teilnahme an wissenschaftlicher Weiterbildung im Alter, steht ganz klar die Befriedigung individueller Bildungsinteressen und der Wunsch, "geistig fit bleiben ${ }^{\text {“18 }} \mathrm{zu}$ wollen, im Vordergrund. Altruistische Motive (z. B. ehrenamtliches Engagement) werden zwar durchweg genannt, spielen aber eine eher nachgeordnete Rolle. Auch das Motiv, Bildung nachzuholen, für die früher keine Zeit war oder zu der kein Zugang bestand, scheint mehr und mehr in den Hintergrund zu treten. Insgesamt haben die Teilnehmenden eine hohe intrinsische Motivation und messen der Bildung einen hohen und zweckfreien Eigenwert bei.

- Neben der Teilhabe an wissenschaftlichen Lehrveranstaltungen ist für gut ein Drittel der Teilnehmenden der gemeinsame Vorlesungs- bzw. Seminarbesuch mit jüngeren Studierenden wichtig. Allerdings kommt es nicht in gleichem Maße zu einem intergenerativen Austausch oder intergenerativer Zusammenarbeit. Mehrfach wird angeführt, dass die Curricula seit der Bologna-Reform der Studiengänge zu restriktiv seien und damit Raum und Zeit für die Zusammenarbeit mit Jüngeren für beide Seiten eher eingeschränkt sind. In den Ergebnisberichten der Studien wird denn auch von den Auswertenden häufig die Sorge artikuliert, dass die Bologna-konformen Studiengänge zu einer Desintegration der Älteren im akademischen Lehrbetrieb und einer Segregation dieser Zielgruppe in eigens für sie geschaffene Veranstaltungsformate führen können. Die Förderung intergenerativer Kontakte und Zusammenarbeit (vgl. Franz 2014) kann sicher als eine Zukunftsaufgabe angesehen werden, zumal in einigen Studien Jüngere wie Ältere äußern, überwiegend gut bis sehr gut miteinander auszukommen und sich gegenseitig zu respektieren.

- Bei den Lernstilen legen die Teilnehmenden ein zunehmend rezeptives Verhalten an den Tag, die Vorlesung ist das bevorzugte Veranstaltungsformat, das Zuhören und Mitschreiben die bevorzugte Beteiligungsform. Eine praktische Konsequenz dieses Ergebnisses könnte sein, partizipativere Lernstile und die Bereitschaft zu aktiver Mitarbeit und eigenen Beiträgen gerade im Hinblick auf intergenerative Zusammenarbeit und die Hebung von Erfahrungsschätzen der Älteren zu fördern.

- Wenig erforscht ist bisher die Haltung der Lehrenden gegenüber den Älteren. Ihnen wird zwar von den Älteren eine hohe Wertschätzung entgegengebracht und eine hohe Fachkompetenz zugesprochen, die Einstellungen der Lehrenden selbst sind bisher aber bestenfalls aus informellen Gesprächen ableitbar. Eine

18 In genau dieser Formulierung ist das Item in Fragebögen mehrerer Standorte enthalten. 
Wertschätzung der Älteren und ihrer Wissens- und Erfahrungsressourcen wird durchaus genannt, ihre Teilhabe in der Regel befürwortet, jedoch stehen dieser Zwänge der Bologna-Administration und daraus folgende zeitliche Engpässe häufig im Wege.

Insgesamt belegen die Befunde im Zeitverlauf eine hohe Stabilität der Zielgruppen. Extreme Entwicklungen sind nicht nachweisbar, interessant bleiben die Veränderungen im Detail, wie z. B. Längsschnittvergleiche unterschiedlicher Standorte oder regionale bzw. standortbezogene Unterschiede der Angebotsstruktur und ihre Auswirkungen auf die Teilnehmerschaft (wobei die umgekehrte Perspektive von Auswirkungen der Teilnehmerschaft auf die Angebotsstruktur auch zu berücksichtigen wäre). Hierzu bedarf es aber noch differenzierter Auswertungen vorliegender und künftiger Studien.

\section{Was sind die künftigen Herausforderungen für die BAG WIWA?}

Die Verfasser/innen sehen im Wesentlichen drei Aufgabenfelder:

1. Der Weg des konzertierten Vorgehens sollte weiter beschritten werden. Eine Vernetzung und Zusammenarbeit der BAG WiWA-Mitglieder schafft eine Stärkung nach innen und hat Signalwirkung nach außen. Anzustreben ist daher auch die Intensivierung der konzeptionellen Zusammenarbeit. Das bestehende Modell Online-Ringvorlesung kann als Folie dienen für weitere überregionale Angebotsformen und -formate (zum Beispiel Webinare, Online-Universität).

2. Die gemeinsamen Forschungsaktivitäten der BAG WiWA sollten fortgesetzt und ausgebaut werden. Der Anfang 2015 gebildete BAG-WiWA-Arbeitskreis Forschungsfragen und Statistik hat hierfür wertvolle Vorarbeit geleistet, indem er aus einer Vielzahl unterschiedlicher lokaler Erhebungen und Untersuchungen den Grundstock für einen einheitlichen Fragebogen gelegt hat, der zukünftig flächendeckende und vergleichbare Daten liefern kann. Weitere Erhebungen können empirisch fundierte Anregungen für neue Angebotsformate liefern sowie empirisch begründete Argumente für die Sensibilisierung und für den Erhalt von wissenschaftlicher Weiterbildung für Ältere an den Mitgliedshochschulen. Wünschenswert wäre die Entwicklung und Implementierung eines Statistik-Portals, um die gesammelten Daten leicht zugänglich zu machen.

3. Eine wichtige Aufgabe bleibt es, das „Seniorenstudium“ als integralen Bestandteil der Hochschulen zu sichern. Die Frage, ob und inwieweit das Studium für Ältere Bologna-konform gestaltet und ausgerichtet wird, muss beobachtet und geprüft werden. In diesem Zusammenhang stellt sich auch die Frage nach dem Status der Teilnehmenden am Studium für Ältere und den Möglichkeiten ihrer Mitwirkungsrechte.

Die Intergenerativität im Hochschulkontext muss gewahrt bleiben. Eine fortschreitende Segregation des Studiums für Ältere muss identifiziert und Lösungen entge- 
gengestellt werden, sodass das bestehende Nebeneinander von integrativen (geöffnete Lehrveranstaltungen) und/oder additiven Formaten (spezielle Veranstaltungen für Ältere) auch weiterhin gewährleistet wird.

In diesem Sinne versteht sich die BAG WiWA zugleich als Forum und Faktor für eine aktive Mitgestaltung von Forschung, Lehre und Studienangeboten zur wissenschaftlichen Weiterbildung im Alter an Hochschulen.

\section{Literatur}

Becker, S./Veelken, L./Wallraven, K. P. (Hrsg.) (2000): Handbuch Altenbildung. Theorien und Konzepte für Gegenwart und Zukunft. Opladen: Leske und Budrich.

Dabo-Cruz, S. (2006): Zwischen Wunsch und Wirklichkeit. Forschungserfahrungen und Perspektiven zur Integration von älteren Studierenden. In: Hochschule und Weiterbildung, 2, S. 33-35.

Dabo-Cruz, S. (2015): Die Universität als Lernort für das 3. Lebensalter. In: Prömper, H./ Richter, R. (Hrsg.): Werkbuch neue Altersbildung. Praxis und Theorie der Bildungsarbeit zwischen Beruf und Ruhestand. Bielefeld: W. Bertelsmann Verlag, S. 259-269.

Franz, J. (2014): Intergenerationelle Bildung. Lernsituationen gestalten und Angebote entwickeln. Bielefeld: W. Bertelsmann Verlag.

Keil, S./Brunner, T. (Hrsg.) (1998): Intergenerationelles Lernen. Eine Zielperspektive akademischer Seniorenbildung. Grafschaft: Vektor-Verlag.

Malwitz-Schütte, M. (Hrsg.) (2000): Selbstgesteuerte Lernprozesse älterer Erwachsener im Kontext wissenschaftlicher Weiterbildung. Bielefeld: W. Bertelsmann Verlag.

Meyer-Wolters, H./Haller, M./Pietsch-Lindt, U./Costard, A. (Hrsg.) (2012): Alter forscht! Forschungsaktivitäten im Seniorenstudium. Forschendes Lernen, Aktionsforschung und Ageing Studies. DGWF-Beiträge 51. Hamburg.

Sagebiel, F. (2006a): Demographischer Wandel und Bolognaprozess. Auswirkungen auf das Seniorenstudium? In: Hochschule und Weiterbildung, 2, S. 9-19.

Sagebiel, F. (2006b): SeniorInnenstudium. In: Faulstich, P. (Hrsg.): Öffentliche Wissenschaft. Neue Perspektiven der Vermittlung in der wissenschaftlichen Weiterbildung. Bielefeld: Transcript-Verlag, S.198-210.

Sagebiel, F./Dahmen, J. (2009): Die Erforschung der Ist-Situation von Studienangeboten für Ältere an deutschen Hochschulen. DGWF-Beiträge 48. Hamburg.

Saup, W. (2001): Studienführer für Senioren. BMBF, Bonn.

Stadelhofer, C. (1998): Lernen in der Informationsgesellschaft. Nutzung und Nutzen des Internets für das weiterbildende Studium älterer Menschen. In: Malwitz-Schütte, M. (Hrsg.): Lernen im Alter. Münster: Waxmann, S. 149-176.

Stadelhofer, C. (2009): Europäische Zusammenarbeit über das Internet als neue Arbeitsund Lernform im Seniorenstudium. In: Hochschule und Weiterbildung, 1, S. 53-56. 
Swindell, R./Thompson, J. (1995): An international perspective on the university of the third age. In: Educational Gerontology 21 (5), S. 429-447.

Tippelt, R. (2009): Bildung Älterer. Chancen im demografischen Wandel. Bielefeld: W. Bertelsmann Verlag.

Zahn, L. (1993): Die akademische Seniorenbildung. Eine historische Bilanz in methodisch-didaktischer Absicht. Weinheim: Deutscher Studien Verlag.

Zentrum für Wissenschaftliche Weiterbildung Oldenburg (Hrsg.) (1980): Öffnung der Universitäten für ältere Erwachsene. Internationaler Workshop an d. Univ. Oldenburg 05.-07. Dezember 1979. 


\title{
Profilbildung und Professionalisierung durch Forschung. Die Arbeitsgemein- schaft Forschung
}

Wolfgang JÜtTe/Maria KondRatjuk/Mandy Schulze

\begin{abstract}
Die Zunahme von Förderprogrammen, Formaten, Projekten und auch Personal in der Weiterbildung an Hochschulen führt zu neuen wissenschaftlichen Fragestellungen. Die Transparenz, Verortung und Systematisierung dieser Fragen und ihrer Beantwortung durch Forschungsarbeiten ist das Anliegen der AG Forschung innerhalb der DGWF. Der vorliegende Beitrag fasst die Zielsetzungen, die Aktivitäten und Aufgaben der Arbeitsgruppe zusammen. Dabei wird vor allem auf das jährliche Forschungsforum als offenes Diskussionsformat mit Werkstattcharakter eingegangen.
\end{abstract}

\section{Genese}

Die Arbeitsgemeinschaft Forschung ist die jüngste der vier Arbeitsgemeinschaften in der DGWF. Sie startete als eine Initiative mit dem Ziel, den Erfahrungsaustausch und die Verständigung über Forschung in und über die Hochschulweiterbildung zu stärken. Vonseiten der DGWF und ihrem Vorstand bestand schon länger der Bedarf, der wissenschaftlichen und forschenden Auseinandersetzung mit Hochschulweiterbildung einen geeigneten organisatorischen Rahmen zu geben. Auf der Jahrestagung 2011 in Bielefeld wurde dieses Bemühen unterstrichen; dabei die Impulse zum DGWF-Forschungsprojekt zur Reorganisation der wissenschaftlichen Weiterbildung aufgreifend, das maßgeblich von Joachim Ludwig (vgl. dazu Dollhausen/Ludwig/ Wolter 2013) initiiert wurde. Damit war der Grundstein für eine AG Forschung in der Fachgesellschaft gelegt und sodann auf der Jahrestagung in München 2012 die Arbeitsgruppe Forschung offiziell gegründet. ${ }^{1}$ Als übergeordnete Zielsetzungen wurden formuliert:

1 Als Sprecher wurden Wolfgang Jütte und im Sprecherrat Maren Kreuz, Maria Kondratjuk, Mandy Schulze und Markus Walber gewählt. 
- die Unterstützung nachhaltiger Entwicklung und Profilierung des Forschungsfeldes Hochschulweiterbildung,

- die Systematisierung der Forschungsaktivitäten im Feld der Hochschulweiterbildung,

- die Förderung des Wissens- und Erfahrungsaustausches zu laufenden Qualifizierungs- und Forschungsarbeiten und

- die Förderung des wissenschaftlichen Nachwuchses, des kollegialen Diskurses und der Vernetzung im Feld.

Die Zielstellungen begründen sich mit dem aktuellen Stand der Hochschulweiterbildung, die aufgrund der zunehmenden Angebote und Formate ein weites Forschungsfeld bietet. Vor dem Hintergrund der projektförmigen Arbeit mit befristeten Beschäftigungsverhältnissen und knappen Projektmitteln fehlen jedoch die institutionellen Rahmenbedingungen, um sich an den Hochschulen als Aufgabenfeld zu verorten. Dieses Desiderat stellt eine Entwicklungsaufgabe dar: ein „forschender Blick“ auf die Entwicklung der Praxis von Hochschulweiterbildung fördert ihre Professionalisierung. Dies drückt auch Karl Weber, der die Arbeit der AG Forschung von Beginn an unterstützte und stets wertschätzend und doch kritisch kommentierte, in einem Interview aus. Dabei verweist er auf die „Expansion der Forschung“:

„Wissenschaftliche Weiterbildung ist nicht nur ein Handlungsfeld der Aktion, sondern inzwischen auch und vermehrt Gegenstand der wissenschaftlichen Reflexion. Dies dokumentiert beispielsweise auch die AG Forschung. Dank Forschung ist es möglich, distanzierter mit den Untiefen in der Praxis der Wissenschaftlichen Weiterbildung umzugehen. Diese Möglichkeit ist meines Erachtens wirklich neu." (Weber 2016, S. 289-290).

\section{Professionalisierung}

Um eine Beforschung des eigenen Feldes anzuregen und NachwuchswissenschaftlerInnen in ihrem Interesse an der thematischen Auseinandersetzung mit der Hochschulweiterbildung zu unterstützen, setzt die Arbeitsgemeinschaft Forschung ein Programm von Aktivitäten um, die der Professionalisierung und Profilierung des Feldes dienen.

Kernstück bildet das jährlich stattfindende „Forschungsforum wissenschaftliche Weiterbildung". Seit Juni 2012 treffen sich in Bielefeld im Umfeld des etablierten universitären Instituts für wissenschaftliche Weiterbildung (IWW) unter der Leitung von Wolfgang Jütte interessierte ForscherInnen und (Nachwuchs)wissenschaftlerInnen zu aktuellen Themen.

Das Forschungsforum dient vor allem der Vernetzung der NachwuchswissenschaftlerInnen und der forschenden AkteurInnen untereinander, die in ihren Qualifizierungsarbeiten oder Forschungsprojekten das Feld der Hochschulweiterbildung be- und erforschen. Hierzu werden in Form eines Werkstattgesprächs Forschungsarbeiten präsentiert und hinsichtlich ihrer methodischen, thematischen und methodo- 
logischen Herangehensweise gemeinsam diskutiert. Wichtige Elemente dieses Austausches sind neben dem kollegialen Charakter der integrative Ansatz, der auch NachwuchswissenschaftlerInnen einlädt, die erst am Anfang einer Qualifizierungsarbeit im Feld stehen. Hierbei wurden Anleihen an das Format der „Werkstatt Forschungsmethoden“ der Sektion Erwachsenenbildung der DGfE genommen, die seit 2003 als Form der Nachwuchsförderung jährlich stattfindet.

Daneben dient das Forschungsforum auch der Vernetzung mit „etablierten“ WissenschaftlerInnen im Feld. Zu Themen aus der aktuellen Forschung wurden u.a. bereits eingeladen: Karin Dollhausen, Carola Iller, Rita Meyer, Joachim Ludwig, Ingeborg Schüssler, Uwe Wilkesmann und Andrä Wolter. Neben dem inhaltlich-fachlichen Austausch werden auch Kooperationen mit benachbarten Fachgesellschaften gesucht, wie der Sektion Erwachsenenbildung der DGfE und der Gesellschaft für Hochschulforschung.

Konstituierend für das Forschungsforum ist, dass die Beiträge weitestgehend dem Bereich „Weiterbildung im Kontext der Hochschule bzw. Hochschulforschung“ zugeordnet werden. Innerhalb dieses Austausches werden unterschiedliche disziplinäre Schnittstellen nicht nur sichtbar, sondern aktiv zur Diskussion gebracht. So zeigen sich die vorgestellten Projekte und Forschungsvorhaben in einer breiten Vielfalt. Perspektiven, die z. B. aus der Hochschulforschung oder der Erwachsenenbildungs-/ Weiterbildungsforschung oder der Lehr-/Lernforschung eingenommen werden, erfahren eine Einordnung in den Kontext der Hochschulweiterbildung. Damit können blinde Flecken in der Forschungslandschaft identifiziert und Diskurse jenseits des „Theorie-Praxis-Gaps“ geführt werden. Die dadurch entstehenden Anregungen und sichtbar gewordenen Synergien dienen als Grundlage für die thematische Ausrichtung der AG Forschung. So werden die Ergebnisse des jährlichen Austausches auf der DGWF-Jahrestagung in einem eigenen Vorseminar, als einem weiteren Veranstaltungsformat, vorgestellt und mit den PraktikerInnen und WissenschaftlerInnen der Community diskutiert. In dem von aktivierenden Methoden unterstützten Seminar werden Impulse des überaus anregenden Austausches in die DGWF und vor allem die AG Forschung zurückgespiegelt, diskutiert und im Gegenzug Forschungsbedarfe aus der Praxis aufgegriffen.

Durch diese beiden Maßnahmen, das zweitätige Forschungsforum in Bielefeld und das Vorseminar auf den Jahrestagungen, und ihren sehr offenen und einladenden Charakter werden nicht nur die im Feld der Hochschulweiterbildung Tätigen über aktuelle Forschungsansätze informiert und NachwuchswissenschaftlerInnen auf Fragen der Praxis aufmerksam. Neben der erhöhten Sichtbarkeit von Forschung im Feld, sowohl im Rahmen von Drittmittelprojekten wie auch von externen Qualifikationsarbeiten, findet eine Systematisierung der Forschungsaktivitäten statt, welche durch die Themenwahl und die Organisation der Aktivitäten der AG Forschung bewusst unterstützt und strukturiert vorangetrieben wird. Das Verfassen von Tagungsbeiträgen oder von Rezensionen in der Zeitschrift „Hochschule und Weiterbildung“ 
(ZHWB) und auch durch Unterstützung der Redaktionsarbeit durch Mitglieder der AG Forschung runden die Kommunikation in die Fachgesellschaft hinein ab.

\section{Systematisierung von Forschung}

Das erste Forschungsforum im Juni 2012 diente der AG Forschung zum einen als Auftakt für die folgenden Aktivitäten und zum anderen als konstituierendes Moment, sich als eine unabhängige Arbeitsgruppe innerhalb der DGWF zu etablieren. Vorträge zu Forschungsarbeiten, die im Rahmen von Qualifikationsarbeiten entstanden, und Vorträge zu abgeschlossenen Begleitforschungsprojekten und Studien etablierter WissenschaftlerInnen ließen ein erstes Bild der Forschung in der Hochschulweiterbildung zeichnen. Ein Diskurs zur Systematisierung der Forschung in und über die Hochschulweiterbildung wurde begonnen (vgl. dazu Kondratjuk/ Schulze 2016).

Das zweite Forschungsforum im Frühjahr 2013 griff diese - durch einen bewilligten Antrag durch den DGWF Vorstand nun offizielle - Konstituierung auf und etablierte das Forschungsforum als offenes Diskussionsforum mit Werkstattcharakter. Durch Einbindung der Forschungsprojekte, die im Rahmen der Initiative „Aufstieg durch Bildung: Offene Hochschule“ liefen, konnte eine erste grobe Skizze der Forschungslandschaft Hochschulweiterbildung entworfen werden. Eine systematisierende $\mathrm{Zu}$ sammenführung der Erkenntnisse des Forums mündeten in einem Panel auf der DGWF-Jahrestagung in Rostock 2013 zum Thema „Forschungsperspektiven und Beobachtungen der wissenschaftlichen Weiterbildung als Beitrag zur Profilierung des Feldes“. Aus der Vorstellung aktueller Qualifizierungsarbeiten im Bereich der Akteure und Programme der Hochschulweiterbildung wurden Thesen für die weitere Diskussion formuliert (vgl. dazu Kondratjuk/Schulze 2014). Abschließend wurde die Frage nach einer „Brücke zwischen Forschung und Praxis“ in der Hochschulweiterbildung diskutiert.

Das dritte Forschungsforum 2014 mit dem Titel „Erste Schritte zur Kartografierung der wissenschaftlichen Weiterbildung" thematisierte Forschungsschwerpunkte und „blinde Flecken“ im Feld der Hochschulweiterbildungsforschung. Im Ansatz einer Kartografierung wurde mit den Teilnehmenden und deren konkreten Forschungsvorhaben versucht, die Forschungslandschaft in thematischer, theoretischer und methodischer Hinsicht zu skizzieren. Während der Diskussion über mögliche Systematisierungen wurde zum einen die große Vielfalt der Projekte, Referenzen und Zugänge deutlich. Zum anderen zeichneten sich jedoch auch erste „Ballungsräume“ sowie „weiße Flecken“ auf der Landkarte ab. So konnte ein Schwerpunkt in der empirischen Forschung, meist auf Grundlage eigener Primärerhebungen, festgestellt werden. Internationale Perspektiven oder Forschungsvorhaben im Bereich der neuen Medien waren hingegen kaum vertreten.

Mit diesem ersten Ansatz einer Systematisierung wurde auf der DGWF-Jahrestagung in Hamburg 2014 ein Vorseminar mit dem Titel „Hochschulweiterbildung 
(neu) beforschen!“ angeboten. Das Seminar mit Workshop-Charakter widmete sich den verschiedenen Zugängen und Methoden der Forschung in der Hochschulweiterbildung; den wahrgenommenen blinden Flecken und Wagnissen bei der Beforschung sowie den Potenzialen theoretischer Rahmung von Forschung zu Hochschulweiterbildung. Die Diskussion mit den Teilnehmenden bestärkte den Mehrwert, den theoretische Rahmungen zur Erforschung von Hochschulweiterbildung bieten können, und gab zahlreiche Anregungen für eine praxisorientierte Forschung in der Hochschulweiterbildung. Die Forschungsaktivitäten werden als kleinteilig und individuell, bspw. auf einzelne Formate wahrgenommen. Als wichtige Differenz wurde deshalb auf den Kontext von Forschung im Bereich verwiesen, der in der Hochschulweiterbildung oftmals durch Drittmittelprojekte oder in Form von Begleitforschung vorhanden ist.

Diesen Impuls aufnehmend widmete sich das vierte Forschungsforum im April 2015 dem Thema: „Nachhaltigkeit von Projekten?! Spurensuche aus Sicht der Forschung“. Den Fachvortrag von Ingeborg Schüßler aufgreifend, wurden Bezüge zur Hochschulweiterbildung als Forschungsfeld diskutiert. Schlagworte in der Diskussion waren „Wirkung“ und „Erwartung an die Wirkungsforschung“ sowie „Nutzen“ und „Nutzung von Ergebnissen“.

Im Vorseminar mit dem Titel „Hochschuldidaktische Handlungsebenen. Zwischen Analysemethode und Praxisverortung" wurde auf der folgenden Jahrestagung 2015 in Freiburg das von Flechsig/Haller (1975) entwickelte Konzept der didaktischen Handlungsebenen erweitert und als ein systematisierendes Angebot zur Verortung der Hochschulweiterbildung angeboten und daraus resultierende Implikationen auf Praxis und Forschung erarbeitet sowie Handlungsspielräume, Grenzen und Ressourcen thematisiert.

Auch das fünfte Forschungsforum 2016 trug zur Systematisierung von Forschung im Feld der Hochschulweiterbildung bei. Unter dem Titel „Kartografie der Hochschulweiterbildung“ wurde die Frage gestellt, wie sich das Forschungsfeld „vermessen“ lässt und welche Rolle die Qualifikationsarbeiten von NachwuchswissenschaftlerInnen dabei spielen? Wichtige Themengebiete waren die Identitätsbildung und die nachhaltige Steuerung zukünftiger Forschungsprogramme über das Mittel der strukturierten Darstellung von laufenden und abgeschlossenen Forschungsprojekten. Erfahrungen mit der Forschungslandkarte Erwachsenenbildung wurden über einen Vortrag von Joachim Ludwig eingebracht. An welchen Ebenen, Zugängen und Methoden eine Systematisierung des Feldes der Hochschulweiterbildung ansetzen kann, bleibt offen. Ein Ansatz ist die Unterstützung von präsentierten Qualifikationsarbeiten innerhalb der AG Forschung. So könnten möglichen Verzerrungen der häufig als kleinteilig wahrgenommenen Forschung aufgrund von Förderrichtlinien begegnet und innovative Ansätze mit breiten disziplinären Zugängen wahrgenommen werden. 


\section{Nachhaltigkeit}

Nachhaltigkeit zielt auf einen längerfristigen Nutzen für alle Beteiligten ab. In der Hochschulweiterbildung sind das neben den anbietenden Hochschulen mit ihren AkteurInnen, die Fachbereiche/Fakultäten und deren VertreterInnen, die Teilnehmenden sowie die NutzerInnen, das Beschäftigungssystem und seine Bereiche. In vielen Förderrichtlinien wird die Nachhaltigkeit von Konzepten als ein zentrales Qualitätskriterium definiert und zielt auf den Aufbau tragender Strukturen und von Programmen ab. Durch die Aktivitäten, die oftmals projektförmig ausgeführt und angegangen werden, ist die Nachhaltigkeit jedoch nicht selten gefährdet.

Die AG Forschung widmet sich der Nachhaltigkeit in zweifacher Weise:

1. mit Fragen der Nachhaltigkeit von Forschung in der Hochschulweiterbildung;

2. mit Fragen der Nachhaltigkeit in Form einer Institutionalisierung der AG Forschung innerhalb der DGWF selbst.

Zur Frage der Nachhaltigkeit von Forschung resümiert Maren Kreutz in ihrem Tagungsbericht zur Jahrestagung 2015:

„In der Praxis wissenschaftlicher Weiterbildung, insbesondere im Kontext öffentlicher Modellförderung wird viel experimentiert, didaktische Konzepte werden entwickelt und erprobt und nicht selten auf der Basis von Erfahrungsansätzen innoviert. Bisher nahezu unberücksichtigt bleibt jedoch, dass es sich bei der wissenschaftlichen Weiterbildung um ein historisch gewachsenes Feld handelt, in dem bereits Theorieansätze und Forschungsergebnisse vorliegen. Diese werden in den aktuellen Diskursen kaum zur Kenntnis genommen und in Bezug zueinander gesetzt bzw. für die Implementierung von Modellvorhaben umgesetzt. Damit eng verbunden stellt sich auch die Frage der Nachhaltigkeit im Kontext der Weiterbildung an Hochschulen auf eine neue Weise. Eine spannende Aufgabe für die Zukunft wird sein, das Feld der Hochschulweiterbildung insgesamt und vor allem das Thema der Didaktik wissenschaftlicher Weiterbildung stärker forschungsbasiert, theoriegeleitet und praxisnah weiterzuentwickeln. Hierzu liefern die Diskurse um Forschungsprioritäten in der wissenschaftlichen Weiterbildung nach wie vor zentrale Anknüpfungspunkte“ (Kreutz 2015, S. 65).

Der Aufbau nachhaltiger Strukturen in der AG Forschung wird nicht nur mit regelmäßigen Veranstaltungen und der breiten Einladung von NachwuchswissenschaftlerInnen nachgekommen. Explizite Angebote fördern die Vernetzung untereinander, wie z. B. das Angebot einer Schreibwerkstatt zum intensiven gemeinsamen Schreiben an der eigenen Qualifikationsarbeit. 


\section{Vernetzung}

Neben dem Austausch unter NachwuchswissenschaftlerInnen und dem transparenten Austausch innerhalb der DGWF pflegt die AG Forschung einen „intergenerationalen" fachlichen Austausch mit „etablierten“ ExpertInnen und WissenschaftlerInnen, die schwerpunktmäßig in anderen Fachgesellschaften engagiert sind.

Wenn auch Selbstvergewisserung, Identitätsbildung und Profilbildung der Hochschulweiterbildung in Theorie und Praxis durch Forschung Ziele der AG Forschung sind, so geht es nicht um eine Abschottung gegenüber anderen Teildisziplinen, die sich dem Forschungsgegenstand Hochschule oder dem Lernen allgemein widmen. Um Synergien zu erzeugen, fand bereits eines der Treffen in Kooperation mit der wissenschaftlichen Begleitung zum Bund-Länder-Wettbewerb „Aufstieg durch Bildung: offene Hochschule“ statt. Auch auf der kooperativ ausgerichteten Tagung der Netzwerkinitiative „Zwischen Baum und Borke“, die durch die Gesellschaft für Hochschulforschung (GfHf), die Gesellschaft für Evaluation (DeGEval), die Deutsche Gesellschaft für Hochschuldidaktik (dghd), die DGWF sowie den Stifterverband im Frühjahr 2016 ausgerichtet wurde, war die AG Forschung mit einer Arbeitsgruppe vertreten. Der interaktive Workshop mit dem Titel „Forschung in der Hochschulweiterbildung. Ein Angebot von und für NachwuchswissenschaftlerInnen“ wandte sich $u$.a. den Fragen zu, wann WissenschaftlerInnen kein Nachwuchs mehr sind, mit welchen disziplinären Zugängen der Forschungsgegenstand Hochschule beforscht wird und wie Qualifikationsarbeiten in diesen Feldern finanziert sind. Der Blick über den Tellerrand zeigte, dass Forschung einen wichtigen Beitrag zu Professionalisierung und Strukturierung des Feldes und seiner Teilbereiche leisten kann. NachwuchswissenschaftlerInnen übernehmen hier oftmals unbewusst eine Vorreiterrolle, indem sie disziplinäre Grenzen furchtlos überschreiten, sich tabuisierten Grauzonen unbedarft widmen und Forschung gegenüber eine „forsche“ Haltung einnehmen.

\section{Neue Räume der Wissensgenerierung}

Die Forschungsaktivitäten im vorrangig praxisorientierten Handlungsfeld Hochschulweiterbildung stellen sich oftmals dem Vorwurf, Praxis ohne Forschung und Forschung ohne Praxis zu machen. Die AG Forschung ist sensibel gegenüber diesen Trennungen und Spiegelgefechten in dem Feld. Forschung muss mit Disziplinbezug stattfinden, ohne dabei die Praxis zu vernachlässigen. Praxisfeldintegrierende Forschungsansätze (siehe den Beitrag von Ortfried Schäffter in diesem Band) beziehen Theorie und Praxis interdisziplinär aufeinander. Gerade die Forschung in der Hochschulweiterbildung, als ein Beitrag zum lebenslangen Lernen und Wahrnehmungsfenster der Hochschulen für Bedarfe an Praxisforschung zwischen Distribution von Wissen für die Gesellschaft und gleichzeitig der Erzeugung von wissenschaftlichem Wissen für die Entwicklung der Hochschulen, ist ein Paradebeispiel 
für ein neues Verhältnis zwischen der Institution Hochschule und anderen Räumen der Wissensgenerierung (Stichwort "mode 2“).

Forschung über und in Hochschulweiterbildung bedeutet neben einer Professionalisierung von Praxis und Stärkung des professionellen Selbstverständnisses durch Anerkennung in der akademischen Welt auch die Möglichkeit der Betonung dieser besonderen Position zwischen akademischer Welt und beruflichen Kontexten. Dies heißt nicht zuletzt auch Position gegenüber einer zunehmenden Ökonomisierung von Weiterbildung an Hochschulen zu beziehen und Bildungsprozesse für viele $\mathrm{zu}$ ermöglichen.

\section{Literatur}

Dollhausen, K./Ludwig, J./Wolter, A. (2013): Organisation und Re-Organisation wissenschaftlicher Weiterbildung in einer bewegten Hochschullandschaft. In: Hochschule und Weiterbildung, S. 10-13.

Flechsig, K. H./Haller, H. D. (1975). Einführung in didaktisches Handeln: ein Lernbuch für Einzel- und Gruppenarbeit. Stuttgart.

Kondratjuk, M./Schulze, M. (2014): Forschungsperspektiven auf die Praxis von Hochschulweiterbildung: Ein Blick auf Akteure und die Programmorganisation. In: Hochschule und Weiterbildung, H. 1., S. 59-64.

Kreutz, M. (2015): Tagungsbericht zur Jahrestagung der DGWF 23-25. September 2015, Freiburg. In: Hochschule und Weiterbildung, H. 2, S. 64-65.

Schulze, M./Kondratjuk, M. (2016): Forschungsaktivitäten in der Hochschulweiterbildung. Ein Systematisierungsvorschlag als Auftakt einer Kartografie. In: Zeitschrift Hochschule und Weiterbildung (ZHWB), H. 2; S. 12-18.

Weber, K. (2016). Interview. Geführt von W. Jütte. In: Zimmermann, T. E./Jütte, W./ Horváth, F. (Hrsg.): Arenen der Weiterbildung. Bern, S. 269-290. 


\section{Im föderativen Spannungsfeld: die Landesgruppen}





\section{Landesgruppe Baden-Württemberg}

ULRICH WACKER

Landesgruppen sind die regionale Differenzierungsdimension in der Arbeit der DGWF. Während die Arbeitsgemeinschaften der inhaltlichen und institutionellen Heterogenität Rechnung tragen und für diese leistungsfähige Foren bilden, tragen die Landesgruppen der regionalen Heterogenität Rechnung. Aspekte wie unterschiedliche rechtliche Rahmenbedingungen oder die regionalen Vernetzungsschwerpunkte wissenschaftlicher Weiterbildungseinrichtungen mit Akteuren in Wirtschaft und Gesellschaft sind hierfür Beispiele. Der Gründung der Landesgruppe BadenWürttemberg 2008 in Karlsruhe ging ein Vernetzungsprozess auf Initiative von Dr. Birgit Stadler von der Universität Heidelberg voraus, der zur bewussten Entscheidung führte, über die Vernetzung hinaus den Weg eines integrativen Prozesses in der DGWF zu gehen. Ausschlaggebend hierfür waren die institutionellen Strukturen eines etablierten Verbandes, der die Interessen von Akteuren in der wissenschaftlichen Weiterbildung und im Fernstudium bündelt. Ein wichtiges Argument war zudem, dass ein Verband der Stimme der engagierten Hochschulen größeres Gewicht verleiht - ein wichtiger Aspekt mit Blick auf das Ziel der Landesgruppe, auch bei der Ausgestaltung der rechtlichen Rahmenbedingungen Gehör zu finden.

Daneben war eine wichtige Zielsetzung nach der Gründungsphase, der regionalen Differenzierungsstruktur Landesgruppe im Dachverband neue Dynamik zu verleihen. So engagierte sich die Landesgruppe für eine Satzungsänderung der DGWF, die die SprecherInnen der Landesgruppen zu stimmberechtigten Mitgliedern des DGWF-Vorstandes machte. Hierdurch trug die Landesgruppe zu Impulsen für die Gründung weiterer Landesgruppen bei: ein Prozess, der mit der Gründung der Landesgruppe Bayern 2015 und einer damit flächendeckenden Struktur von Landesgruppen in Deutschland seinen Abschluss fand.

\section{Neue Kooperationsstrukturen}

Erster Schritt in Richtung Arbeit an den rechtlichen Rahmenbedingungen der wissenschaftlichen Weiterbildung in Baden-Württemberg war der regelmäßige Austausch mit dem Ministerium für Wissenschaft, Forschung und Kunst Baden-Würt- 
temberg (MWK). Auch wenn wissenschaftliche Weiterbildung nach wie vor einen kleinen Bereich der Arbeit der Hochschulen darstellt, so misst das MWK der strukturellen und strategischen Bedeutung der wissenschaftlichen Weiterbildung zunehmend Gewicht bei. Im Laufe der Jahre verstetigte sich die Zusammenarbeit mit dem Referat Weiterbildung des Ministeriums für Wissenschaft und Kunst - ein für die Netzwerkarbeit der Landesgruppe wichtiger strategischer Baustein. Ausdruck fand diese Zusammenarbeit auch in einem Arbeitskreis gemeinsam mit variierenden Akteuren wie beispielsweise Südwestmetall oder VertreterInnen der Evaluationsagentur Baden-Württemberg zu Themen, die von Abschlussformaten über Qualität und Nachfrageorientierung bis hin zur Akkreditierung reichen. Dieser Arbeitskreis implizierte auch eine verstetigte Zusammenarbeit mit der Kontaktstelle HOCHSCHULEWIRTSCHAFT, einer Initiative von Südwestmetall zum besseren Matching von Angeboten mit der Nachfrage von Unternehmen nach wissenschaftlicher Weiterbildung.

\section{Weiterentwicklung der rechtlichen Rahmenbedingungen}

Im Kontext des regelmäßigen Austauschs mit dem MWK brachte die Landesgruppe auch ihr Anliegen ein, das Format Kontaktstudium vollständig Bologna-kompatibel auszugestalten und zu flexibilisieren. So wurde die DGWF mit ihrer Landesgruppe Baden-Württemberg als Verband 2013 schließlich zum Dritten Hochschulrechtsänderungsgesetz angehört. Ein wichtiger Meilenstein, denn die Landesgruppe hatte die für die Angebotsentwicklung wichtigen Punkte der Bologna-kompatiblen Ausgestaltung der niederschwelligen Zertifikatsstudien sowie des berufsbegleitenden, weiterbildenden Bachelors diskutiert und eingebracht - wichtige Bausteine auch mit Blick auf kumulative Studienmodelle im Rahmen wissenschaftlich fundierten Lebenslangen Lernens. Ergebnis der gesetzlichen Weiterentwicklung waren die angestrebten flexibleren Rahmenbedingungen, die der Entwicklung neuer Studienmodelle und Studienangebote weitere Dynamik verliehen. So können Zertifikatsstudien in BadenWürttemberg sowohl privatrechtlich als auch öffentlich-rechtlich ausgestaltet und mit Kreditpunkten auf der Basis des ECTS bewertet werden. Die kontinuierliche Zusammenarbeit mit dem MWK trug auch dazu bei, dass das Land Baden-Württemberg 2013 das Förderprogramm „Initiativen zum Ausbau berufsbegleitender Masterangebote“ und 2015 das Förderprogramm „Auf- und Ausbau von Strukturen der wissenschaftlichen Weiterbildung an Hochschulen in Baden-Württemberg“ initiierte, um innovative Angebote und Strukturen zu fördern.

\section{Bündnis für Lebenslanges Lernen}

Wissenschaftliche Weiterbildung in Baden-Württemberg begreift sich als das unmittelbar forschungsbasierte Angebot in der Vielfalt der Angebote von Weiterbildung und Lebenslangem Lernen. Am 31. Januar 2011 unterzeichnete die DGWF-Landes- 
gruppe das „Bündnis für Lebenslanges Lernen“, das die Landesgruppe mit erarbeitet hatte. Das Bündnis für Lebenslanges Lernen war ein Ergebnis der Enquetekommission „Fit fürs Leben in der Wissensgesellschaft“. Ziel war, unterschiedlichste Akteure im Bereich der Weiterbildung besser zu vernetzen, deren Arbeit übergeordnet programmatisch zu formulieren und konkrete Projekte, wie zum Beispiel in der Weiterbildungsberatung, zu entwickeln. Wissenschaftliche Weiterbildung in einem umfassenden Verständnis - nicht nur mit Fokus auf arbeitsmarktorientierte wissenschaftliche Weiterbildung - mit der Betonung des Bildungsauftrags der Hochschulen konnte so in den Bündnistext eingebracht werden. Die Arbeit des „Bündnis für Lebenslanges Lernen“ wurde jüngst für den Zeitraum bis $2020 \mathrm{im}$ „Weiterbildungspakt Baden-Württemberg" programmatisch konkretisiert.

Zahlreiche in der Landesgruppe aktive Hochschulen sind auch Mitglied im Netzwerk für Berufliche Fortbildung in Baden-Württemberg, das vom Ministerium für Finanzen und Wirtschaft unterstützt wird. So entstehen neue Schnittstellen zur beruflichen Weiterbildung, und die Angebote der Hochschulen können im InternetPortal von Fortbildung Baden-Württemberg effektiver präsentiert werden.

\section{Diskursbeiträge und Empfehlungen der Landesgruppe}

Eine wichtige Aufgabe sieht die Landesgruppe in der Entwicklung von Positionen zu aktuellen Fragen der wissenschaftlichen Weiterbildung, die sich aktiv in Debatten einmischen. Startpunkt hierfür war 2010 ein Positionspapier zu Qualitätsstandards in der Wissenschaftlichen Weiterbildung, denen sich die Mitglieder der Landesgruppe verpflichteten. Diese Standards wurden bis 2013 als Qualitätsdimensionen weiterentwickelt, die von wissenschaftlicher Weiterbildung als Dienstleistung über die Beziehung von Forschung und Praxis in der wissenschaftlichen Weiterbildung, die Teilnehmenden- und Bedarfsorientierung sowie Transparenz bis hin zu Beratung, Begleitung, Transfersicherung und Feedback-Prozessen reichen. Die Qualitätsdimensionen sind auch ein wichtiger Input für die Integration der wissenschaftlichen Weiterbildung in die institutionelle und programmbezogene Qualitätssicherung der Hochschulen. Vor diesem Hintergrund brachte die Landesgruppe das Thema wissenschaftliche Weiterbildung aktiv in die Debatte um Programm- und Systemakkreditierung an den Hochschulen ein.

Auf den Qualitätsdimensionen aufbauend erarbeiteten die Mitgliedshochschulen 2014 dann ein Positionspapier zum Selbstverständnis und zur Philosophie der Landesgruppe. Die Landesgruppe versteht sich hierbei als aktiver Partner des DGWFBundesvorstandes und begreift sich durch die Förderung Lebenslangen Lernens auf der Basis der in den Hochschulen vorhandenen Kompetenzen als besonderer Intermediär, dessen Arbeit auch auf Rückkoppelungseffekte auf die Strategien und die Organisation der Hochschulen zielt. Ausgehend hiervon erarbeitete die Landesgruppe aufbauend auf früheren Beiträgen der DGWF jüngst Empfehlungen zu den Formaten der wissenschaftlichen Weiterbildung. Mit Bezug auf die im Landes- 
hochschulrecht vorgegebenen Formate und die Kanonisierung wissenschaftlicher Weiterbildungsabschlüsse in der Schweiz wurde die Systematisierung der Zertifikatsabschlüsse in den Anforderungsdimensionen Bachelor- und Masterebene weiterentwickelt - konsequent abgeleitet aus dem Bologna-Prozess. So trägt die Landesgruppe dazu bei, die Vielfalt der wissenschaftlichen Weiterbildungsangebote übersichtlich, transparent und systematisch zu gestalten. Eine wichtige Aufgabe, die sich aus der Diskussion der Formate ergab, ist aus Sicht der Landesgruppe die Positionierung von Zertifikatsstudien im Deutschen Qualifikationsrahmen (DQR).

\section{Arbeitsstrukturen in der DGWF-Landesgruppe}

Voraussetzung einer erfolgreichen Verbandsarbeit ist Transparenz in Verbindung mit verstetigten und funktionierenden Strukturen der Zusammenarbeit. So treffen sich die Mitglieder der DGWF-Landesgruppe in der Regel dreimal im Jahr zu eintägigen Arbeitstreffen. Die Agenda der Arbeitstreffen fokussiert die jeweils aktuellen inhaltlichen Schwerpunkte. Mit dem Ziel von Transparenz und einer effizienten Bereitstellung von Informationen für die Mitglieder der Landesgruppe und insbesondere neue Mitglieder bündelt eine Moodle-Plattform, die unmittelbar nach der Gründung der Landesgruppe etabliert wurde, alle wichtigen Dokumente und Kommunikationsprozesse. So können Diskurse in der Landesgruppe gut nachvollzogen und auf erarbeiteten Input kann jederzeit zurückgegriffen werden. Die Landesgruppe schafft hierdurch auch einen wichtigen Anreiz für Hochschulen, Mitglied in der DGWF und der Landesgruppe zu werden. Entsprechend erfreulich ist die Entwicklung der Zahl der in der Landesgruppe aktiven Mitgliedshochschulen: Hatten 2008 neun Hochschulen die Landesgruppe gegründet, so arbeiten mittlerweile die Weiterbildungseinrichtungen der Universitäten Freiburg, Heidelberg, Konstanz, Mannheim, Stuttgart, Tübingen und Ulm, des Karlsruher Instituts für Technologie, der Hochschulen Albstadt-Sigmaringen, Biberach, Esslingen, Furtwangen, Heilbronn und Pforzheim, der Pädagogischen Hochschulen in Heidelberg, Karlsruhe, Ludwigsburg und Weingarten sowie der Dualen Hochschule Baden-Württemberg, der Hochschule der Medien in Stuttgart, der Evangelischen Hochschule Ludwigsburg, der Graduate School Rhein-Neckar und der SRH Hochschule Heidelberg in der Landesgruppe zusammen.

Daneben trägt die Landesgruppe aktiv zur Arbeit des Gesamtverbandes bei. Ein wichtiger Beitrag sind hierbei Tagungen. Unterstützt durch die Landesgruppe Baden-Württemberg waren 2015 die Universität Freiburg Gastgeber der DGWF-Jahrestagung und 2011 die Pädagogische Hochschule Heidelberg sowie 2014 die Universität Konstanz Gastgeber der AG-E Frühjahrstagung. Auch um regionale Schnittstellen zum Netzwerk Offene Hochschule weiterzuentwickeln, fand im Februar 2016 eine gemeinsame Tagung der Landesgruppe und des Netzwerks in der Pädagogischen Hochschule Karlsruhe statt. Schließlich veranstalteten das MWK, die Kontaktstelle HOCHSCHULEWIRTSCHAFT und die Landesgruppe 2014 und 2015 gemein- 
sam sechs Regionalforen mit einem abschließenden Regionalforum am 8. Mai 2015 in Stuttgart, in dessen Rahmen Ministerin Theresia Bauer den Weiterbildungspreis Baden-Württemberg HOCHSCHULEWIRTSCHAFT für herausragende Angebotskonzepte überreichte. 



\title{
Landesgruppe Bayern
}

\author{
Gabriele Vierzigmann/Michael Renz/Sylvia Derra/ \\ Sybille Barth/Johannes Ries/Volker Stieg
}

Aus der Tatsache, dass die Landesgruppe Bayern als letzte noch fehlende erst im Jahr 2015 gegründet wurde, könnte man schlussfolgern, dass die wissenschaftliche Weiterbildung (WWB) an den bayerischen Hochschulen noch nicht so recht Fuß gefasst habe. Das Gegenteil ist der Fall. In der Landesgruppe Bayern haben sich Hochschulen zusammengefunden, die bereits seit Langem erfolgreich vielfältige weiterbildende Studienangebote in ihrem Portfolio haben, diesen Bereich der Hochschulbildung weiter ausbauen und diesbezügliche Interessen gemeinsam verfolgen wollen. Studierwillige von heute und morgen wollen flexibler und individualisierter studieren. Der Arbeitsmarkt verlangt nach akademischer Weiterqualifizierung während der Berufstätigkeit. Und nicht zuletzt durch entsprechende Änderungen des Bayerischen Hochschulgesetzes, das mittlerweile eine Vielzahl innovativer Studienformate vorsieht und in dem die Weiterbildung als Auftrag der Hochschulen benannt wird, kommt WWB und lebenslangem Lernen an den Hochschulen eine zentrale Stellung neben Forschung, Lehre und Studium zu. Mit der Einführung von berufsbegleitenden (Bachelor- und Master-)Studiengängen, der Öffnung des Hochschulzugangs für qualifizierte Berufstätige, dem aktiven Umgang mit der Diversifizierung von Bildungswegen und der damit verbundenen Heterogenität der Studieninteressierten und Studierenden hat die WWB Einzug in den akademischen Kernbereich unseres alltäglichen Hochschulgeschäfts gehalten. Uns eint die Überzeugung, dass das lebensbegleitende Studieren in unterschiedlichen Phasen der (Berufs-)Biografie und die Verzahnung von akademischer und beruflicher Bildung ausgesprochen zukunftsträchtig sind. Zugleich wissen wir, dass mit dieser Erweiterung der Hochschulbildung enorme Anforderungen verbunden sind und kreative Anpassungen vorgenommen werden müssen, ohne dabei die Qualität und die Besonderheiten der Hochschulbildung aus den Augen zu verlieren.

Im Bayerischen Hochschulgesetz wurde die Weiterbildung in Art. 2 Abs. 2 bereits 1973 als Aufgabe der Hochschulen niedergelegt. Die erste große Reform des Hochschulgesetzes im Jahr 1998 formulierte in Art. 2 Abs. 3 BayHSchG: „Die Hochschulen dienen dem weiterbildenden Studium und entwickeln Veranstaltungen der Weiterbildung. Sie bieten eigene Veranstaltungen an und beteiligen sich an Weiterbildungsangeboten an- 
derer Träger. Sie fördern die Weiterbildung ihres Personals." 2006 wurde die Weiterbildung auf eine Stufe mit Forschung, Lehre und Studium gestellt (Art. 2 Abs. 1 Satz 1 BayHSchG): „Die Hochschulen dienen der Pflege und Entwicklung der Wissenschaften und der Künste durch Forschung, Lehre, Studium und Weiterbildung in einem freiheitlichen, demokratischen und sozialen Rechtsstaat." Davon abgetrennt wurde die Förderung der Weiterbildung des eigenen Personals in Art. 2 Abs. 1 Satz 7 BayHSchG.

Gemäß Bayerischem Hochschulgesetz können sowohl grundständige als auch postgraduale Studiengänge in besonderen Studienformen angeboten werden, z. B. in Teilzeit oder als berufsbegleitendes Format. Eine spezielle und im Bayerischen Hochschulgesetz normierte Form eines Teilzeitstudiengangs ist der berufsbegleitende Studiengang (Art. 56 Abs. 4 BayHSchG). Ein Studiengang darf nur dann das Attribut „berufsbegleitend“ tragen, wenn neben besonderen Vorkehrungen (insbesondere Konzentration der Lehre auf Abendstunden, Wochenenden und Blockkurse sowie Anteile virtueller Lehre) im Studienverlauf nicht mehr als 20 Leistungspunkte pro Semester veranschlagt sind und er somit neben einer vollen Berufstätigkeit studiert werden kann. Die in diesen Studiengängen lehrenden ProfessorInnen können im Haupt- oder im Nebenamt tätig sein; die Hochschulen können für die aufgrund der besonderen Organisationsform zusätzlich entstehenden Aufwände Gebühren erheben. Das Gesetz regelt zudem weiterbildende Masterstudiengänge, die sich an erfahrene Fachkräfte mit einem ersten Hochschulabschluss und mindestens einem Jahr qualifizierter Berufserfahrung richten. Die lehrenden ProfessorInnen sind in der Regel im Nebenamt tätig; der Aufwand der Hochschulen muss sich zur Gänze aus den Teilnehmergebühren refinanzieren.

Das Bayerische Staatsministerium für Bildung und Kultus, Wissenschaft und Kunst fördert die WWB intensiv. Das Modellprojekt „Akademische Weiterbildung stärken“ (2010-2013) hatte den Aufbau eines vernetzten Systems von Angeboten des lebenslangen Lernens an staatlichen bayerischen Hochschulen (Vorkurse, berufsbegleitende Studiengänge, Teilzeitstudiengänge, Weiterbildungsstudiengänge) sowie Konzepte für die überörtliche Vermarktung der Angebote im Bereich des lebenslangen Lernens und WWB zum Ziel. An den von 2010 bis 2015 ausgeschriebenen Landesförderprogrammen haben nahezu alle staatlichen bayerischen Hochschulen partizipiert, 15 Hochschulen wurden mit zwei bzw. drei verschiedenen Projekten gefördert (die fünf bislang nicht geförderten Hochschulen hatten sich nicht an den Ausschreibungen beteiligt). Ein weiteres Beispiel stellt der Campus Wissenschaftliche Weiterbildung Bayern (cwwb) dar, eine Initiative der bayerischen Universitäten und Hochschulen für angewandte Wissenschaften zur gemeinschaftlichen Vermarktung ihrer Weiterbildungsangebote. Das Portal präsentiert unter www.cwwb.de die Weiterbildungsangebote der staatlichen bayerischen Hochschulen in einem Gesamtkatalog.

Gleichwohl sind weiterbildende und berufsbegleitende Studiengänge auch für die Ministerien ein neues Handlungsfeld. Die Regelungsdichte für diese Studiengänge ist enorm und wird laufend aktualisiert. Dadurch entstehen hohe Arbeitsaufwände für die StudiengangentwicklerInnen und die Gremien der Hochschulen, die ihre 


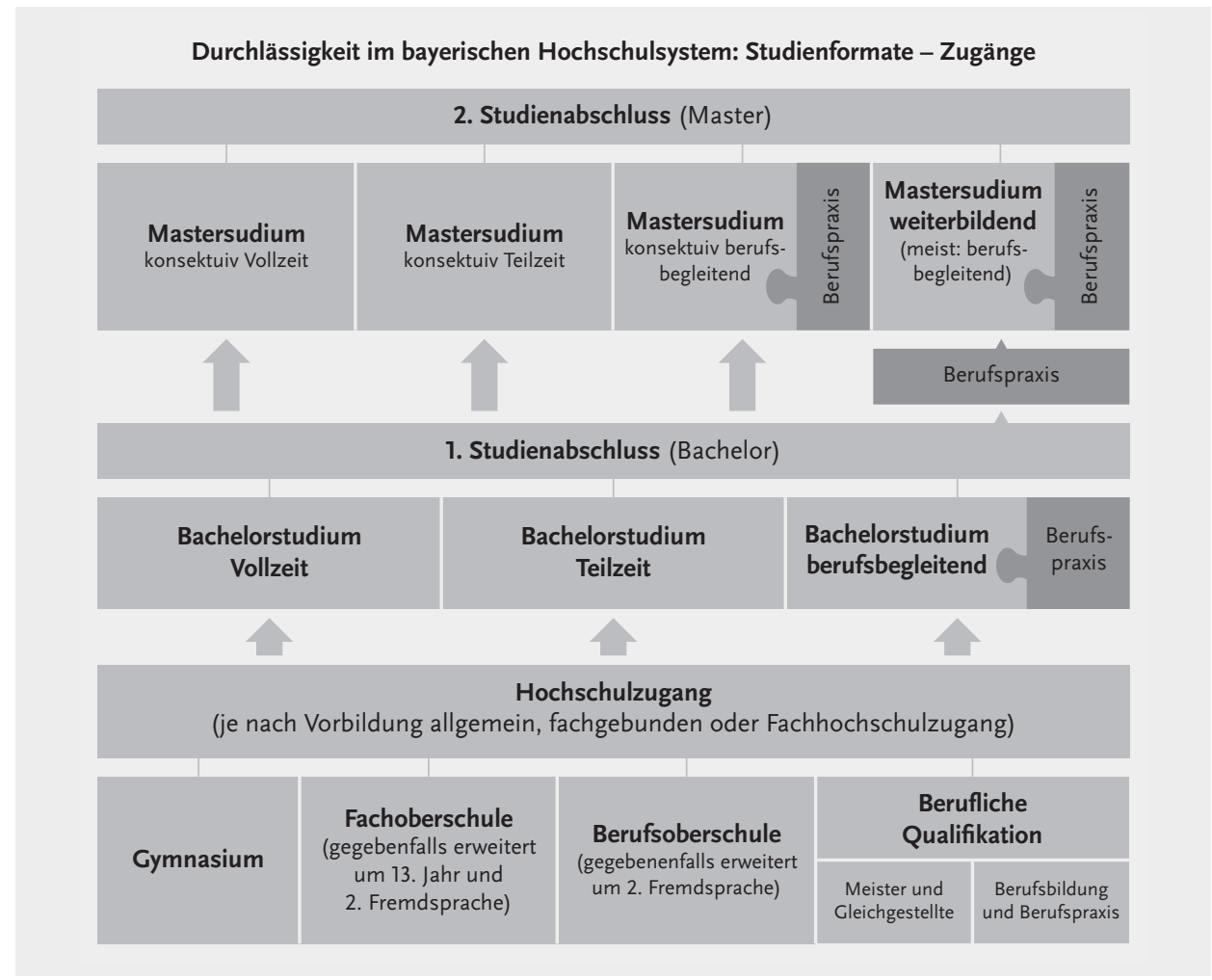

Abb. 1: Durchlässigkeit im bayerischen Hochschulsystem

Quelle: Bayerisches Staatsministerium für Bildung und Kultus, Wissenschaft und Kunst. www.weiter-studieren-inbayern.de

Pilotstudiengänge immer wieder überarbeiten und den neuen Vorgaben anpassen müssen.

Im Kontext dieser Rahmenbedingungen hält die Landesgruppe Bayern ein bildungspolitisches Engagement für angezeigt und hat die Arbeitsgruppen „Gemeinsamkeiten und Besonderheiten: Organisationsformen und Organisationsaufbau WWB an Hochschulen“, „Hochschulen im Spannungsfeld zwischen Bildungsauftrag und Marktteilnahme“ und „Rechts- und Sachfragen“ gebildet. Ziel ist es, entsprechende Thesen einer erfolgreichen WWB an Hochschulen in Bayern zu erarbeiten und diese in Fachdiskussion und Politik einzuspeisen. Die Landesgruppe setzt sich dafür ein, dass für die Förderung der WWB kontraproduktive oder gar widersprüchliche rechtliche Regelungen angepasst werden und von Vorgehensweisen, die in anderen Bundesländern erfolgreich praktiziert werden, gelernt wird. Dazu gehören die Schaffung von Anreizsystemen für Lehrende oder die sich im Spannungsfeld zwischen Markt und Möglichkeiten bewegende Vergütungssituation an Hochschulen, aber auch konkrete Problemstellungen wie Fragen der ORH-konformen Trennungsrech- 
nung oder die (Un-)Möglichkeit, die Tätigkeit als Studiengangleitung separat zu vergüten. Auch ist die vor allem für nicht-traditionelle Studierende nötige Vorbereitungsphase derzeit nur mithilfe externer Bildungspartner abbildbar. Die Hindernisse bei der Umsetzung berufsbegleitender Bachelorprogramme, die einen geringeren Spielraum bei der Gebührenerhebung zulassen, sind in diesem Zusammenhang ebenfalls zu nennen ( $\mathbb{2}$ Absatz 4 HSchGebV).

Die Landesgruppe sieht weiterbildungsaktive Hochschulen als lebenslange Bildungspartner und fordert deshalb ausreichende Planstellen und die Sicherstellung einer (Grund-)Finanzierung ebenso wie die Schaffung von Freiräumen für unternehmerisches Handeln in der WWB. Die Forderungen der Positionspapiere des Universität Bayern e.V. sowie des Hochschule Bayern e. V. aus den Jahren 2013 und 2014 zur (Grund-)Finanzierung der bayerischen Hochschulen sind noch lange nicht erreicht. Gleichwohl wird den Hochschulen durch die Innovationsbündnisse ermöglicht, individuelle Schwerpunkte zu gestalten; ein Ansatz, der auch zur Förderung der WWB zukunftsträchtig erscheint.

Zudem erforscht die Landesgruppe derzeit, welche Organisationsformen und strukturellen Gegebenheiten der WWB an bayerischen Hochschulen zu finden sind bzw. sich bewährt haben und analysiert ausgehend von den Empfehlungen der DGWF (2015) zur "Organisation der wissenschaftlichen Weiterbildung an Hochschulen“, was erfolgsträchtige akademische Organisationsformen auszeichnet. Durch die KMW wurde bereits um die Jahrtausendwende die Empfehlung zum Aufbau zentraler Einrichtungen für die Weiterbildung an Hochschulen ausgesprochen (vgl. KMK 2001, S. 6 f.). Da der Gesetzgeber weitestgehend auf eine Normierung der Organisa-

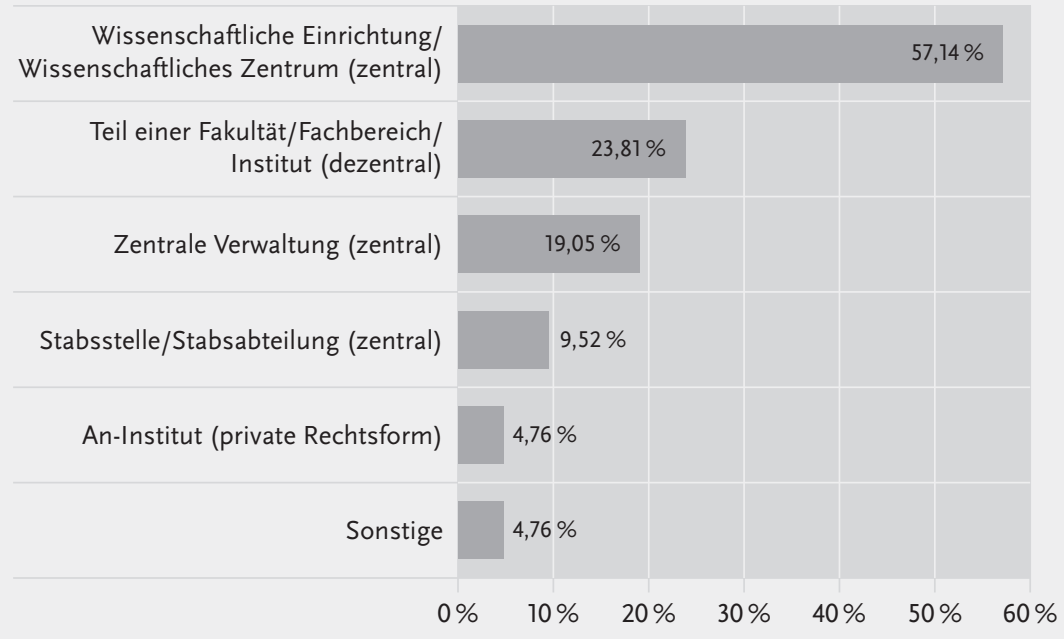

Abb. 2: Organisationsformen wissenschaftlicher Weiterbildung an bayerischen Hochschulen $(n=20)$

Quelle: Eigene Darstellung 
tion von WWB verzichtete, lässt sich in der aktuellen Hochschulpraxis eine Vielzahl unterschiedlicher Ansätze und Erscheinungsformen der organisationalen Einbettung ausmachen (z.B. Hanft/Knust 2007; Wilkesmann 2007; Faulstich/Oswald 2010; Hanft 2014; Vierzigmann/Pohlmann 2016). Bei einer Bestandserhebung zur derzeitigen Organisation und Struktur der WWB in Bayern, an der sich 20 der aktuell 27 der Landesgruppe angeschlossenen Hochschulen beteiligten, bestätigte sich dieser Befund. In der bayerischen Hochschullandschaft sind zentrale Einrichtungen in der WWB zwar verbreitet, aber keinesfalls flächendeckend umgesetzt. So organisieren $24 \%$ der bayerischen Hochschulen ihre Weiterbildungsaktivitäten dezentral, z. B. als Teil einer Fakultät oder eines Fachbereichs (inkl. Mehrfachnennungen). Zudem wird eine starke Heterogenität sichtbar: In Bayern lassen sich Vertreter für insgesamt fünf unterschiedliche Organisationsformen finden. Eine Hochschule ordnet ihre Form sogar außerhalb der angegebenen, üblichen Schemata ein (siehe Abbildung 2). Weiterhin stellt sich bei unserer Befragung heraus, dass nur zwei Hochschulen bei der Organisation ihrer WWB mit einer Stabsabteilung arbeiten. Das Selbstverständnis der bayerischen Hochschulweiterbildungsorganisationen scheint eher intermediär ausgerichtet zu sein (61,1\%). Gerade einmal 16,7\% bzw. 22,2\% der befragten WeiterbildungsvertreterInnen sehen ihre Organisation als primär administrativ oder als primär wissenschaftlich ausgerichtet. Warum sich zentrale Verwaltungsstrukturen bislang nicht durchsetzen konnten bzw. als wenig zielführend erachtet werden, sollen weitere Analysen zeigen.

Die Anzahl der Studierenden in weiterbildenden und berufsbegleitenden Studiengängen zeigt keinen signifikanten Zusammenhang mit der Größe der Hochschule. Im Schnitt kommt an den befragten bayerischen Hochschulen auf 40 StudentInnen bereits ein Weiterbildungsstudent. Die vergleichbar hohen Teilnehmerzahlen dokumentieren, dass eine erfolgreiche Positionierung auf dem Weiterbildungsmarkt mit

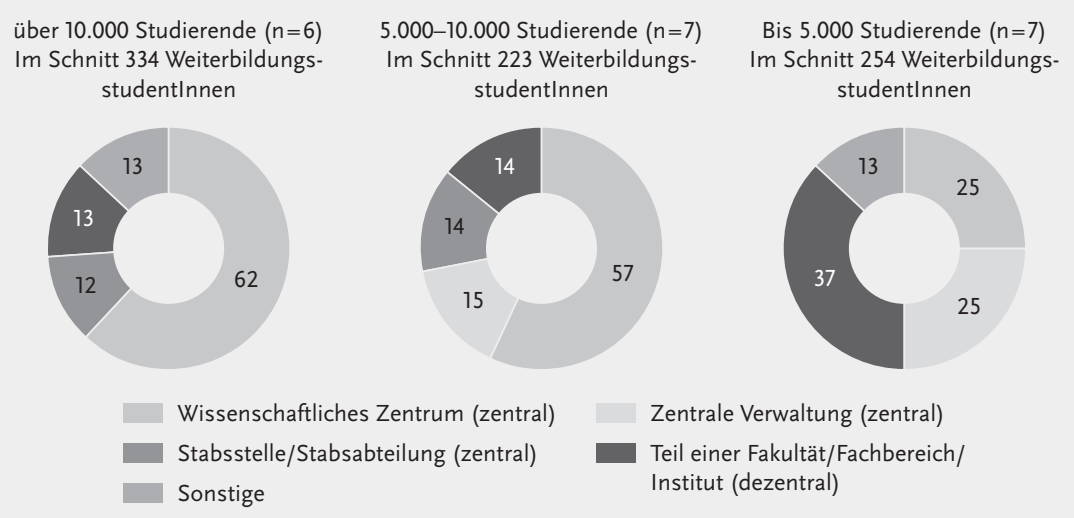

Abb. 3: Organisationsformen wissenschaftlicher Weiterbildung in Zusammenhang mit Größe der Hochschule und Anzahl der WeiterbildungsstudentInnen an bayerischen Hochschulen $(n=20)$

Quelle: Eigene Darstellung 
individuellen Organisationskonzepten und regional unterschiedlichen Lösungen möglich ist. Es kann jedoch beobachtet werden, dass dezentrale Organisationsformen eher an kleineren Hochschulen zu finden sind. Größere Hochschulen setzen vollständig auf An-Institute und zentrale Organisationseinheiten. Nur $12 \%$ der befragten Hochschulen geben an, Tätigkeiten und Aufgabengebiete der WWB aus der Hochschule auszulagern. Hier zeigt sich, dass WWB als eine originäre Aufgabe begriffen wird, die eigenverantwortlich und zunehmend professionell wahrgenommen wird.

Bayerische Hochschulen und der BMBF-Wettbewerb „Aufstieg durch Bildung: offene Hochschulen" sind eine zukunftsweisende Verbindung eingegangen: Vier der neun staatlichen Universitäten (Bayreuth, Erlangen-Nürnberg, Passau und TU München) und acht der 17 staatlichen Hochschulen (Amberg-Weiden, Aschaffenburg, Deggendorf, Ingolstadt, Kempten, Landshut, München und Regensburg) sind an entsprechenden Projekten beteiligt. Für die weitere Arbeit der Landesgruppe soll eine möglichst enge Netzwerkbildung eben jener Projekte mit den VertreterInnen der WWB auf der Agenda stehen, um Wissenstransfer und Synergieeffekte für die WWB zu nutzen.

Die Landesgruppe Bayern geht von einem geschlossenen Regelkreis zwischen Forschung und Weiterbildung aus, den es zu entwickeln gilt: Aus der Forschung heraus werden Ansätze und Ergebnisse generiert, die im Rahmen der wissenschaftlichen Weiterbildung für die Lösung zielgruppenspezifischer Praxisprobleme genutzt werden. Im Kontext der Weiterbildung werden durch die TeilnehmerInnen angewandte Fragestellungen aufgeworfen, die zum Gegenstand der Forschung werden können. Weiterbildung versteht sich somit als Motor für Forschung und kann schließlich selbst zum Gegenstand der Forschung werden, z. B. indem Ansätze, Methoden und didaktische Szenarien im Hinblick auf ihren Beitrag zum Bildungserfolg untersucht werden. Im Wechselspiel zwischen Realität und Rahmenbedingungen sieht sich die Landesgruppe Bayern dabei als wichtiger Rat- und Impulsgeber sowohl für die beteiligten als auch für externe AkteurInnen, um dem gemeinsamen Ziel der Förderung wissenschaftlicher Weiterbildung in Bayern immer noch ein Stück näherzukommen.

\section{Literatur}

DGWF (2015): Organisation der wissenschaftlichen Weiterbildung an Hochschulen. DGWF-Empfehlungen beschlossen am 16./17.07.2015 in Oestrich-Winkel. https://dgwf. net/fileadmin/user_upload/DGWF/DGWF-Empfehlungen_Organisation_08_2015.pdf [Zugriff: 25.01.2015] 
Faulstich, P./Oswald, L. (2010): Wissenschaftliche Weiterbildung, Hans Böckler Stiftung, Arbeitspapier 200.

Hanft, A. (2014): Management von Studium, Lehre und Weiterbildung an Hochschulen. Münster: Waxmann.

Hanft, A./Knust, M. (Hrsg.) (2007): Weiterbildung und lebenslanges Lernen an Hochschulen. Eine internationale Vergleichsstudie zu Strukturen, Organisation und Angebotsformen. Münster: Waxmann.

KMK (2001): Sachstands- und Problembericht zur „Wahrnehmung wissenschaftlicher Weiterbildung an Hochschulen“ - Beschluss der Kultusministerkonferenz vom 21.09.2001. http://www.kmk.org/fileadmin/Dateien/veroeffentlichungen_beschluesse/ 2001/2001_09_21-Problembericht-wiss-Weiterbildung-HS.pdf [Zugriff: 25.01.2015]

Tannapfel, S./Greger, R. (2009): Wissenschaftliche Weiterbildung. In: Geis, M.-E. (Hrsg.): Hochschulrecht im Freistaat Bayern - Handbuch für Wissenschaft und Praxis. Heidelberg: C. F. Müller, S. 238-243.

Vierzigmann, G./Pohlmann, S. (2016): Wissenschaftliche Weiterbildung organisieren. In: Pohlmann, S./Vierzigmann, G./Doyé, T. (Hrsg.): Weiterdenken durch wissenschaftliche Weiterbildung. Impulse aus dem Bundesprogramm Aufstieg durch Bildung: offene Hochschulen. Berlin, Heidelberg: Springer VS.

Wilkesmann, U. (2007): Die Organisation der Weiterbildung. Discussion Papers, Zentrum für Weiterbildung der Technischen Universität Dortmund. http://dx.doi.org/10.17877/ DE290R-7317 [Zugriff: 22.01.16] 



\title{
Landesgruppe Berlin und Brandenburg
}

Peer-Olaf Kalis/Annette Strauß

\begin{abstract}
Die DGWF-Landesgruppe Berlin und Brandenburg war die erste, 1993 gegründete Landesgruppe des damaligen Arbeitskreises Universitäre Erwachsenenbildung (AUE). Ihr wichtigstes strategisches Ziel ist die kontinuierliche Stärkung der wissenschaftlichen Weiterbildung in den jeweiligen Hochschulen und Universitäten. Aktuelle Arbeitsfelder sind die intensive Zusammenarbeit mit den Projekten des Förderprogramms „Aufstieg durch Bildung: offene Hochschulen“ sowie die Integration der Geflüchteten.
\end{abstract}

Die DGWF-Landesgruppe Berlin und Brandenburg war die erste Landesgruppe des damaligen Arbeitskreises Universitäre Erwachsenenbildung (AUE) überhaupt. Ihre Gründung am 4. März 1993 erfolgte im Nachgang der sehr intensiven Kontakte zwischen dem Arbeitskreis Universitäre Weiterbildung der DDR (AUW) und dem AUE. In Berlin und Brandenburg fand sich ein großes Potenzial für eine kontinuierliche Zusammenarbeit, die Freie Universität und die Humboldt-Universität zu Berlin waren hier die Hauptakteure der ersten Stunde, nach kurzer Zeit ergänzt um die Verantwortlichen für die wissenschaftliche Weiterbildung der Technischen Universität Berlin und der damaligen Hochschule der Künste (heute Universität der Künste).

Hauptaufgabe der Landesgruppe war damals die Unterstützung der Aktivitäten der wissenschaftlichen Weiterbildung in der Region Berlin und Brandenburg und vor allem die Zusammenführung der unterschiedlichen Akteure in der Region. Ein wichtiges Instrument war der kontinuierliche Austausch, der in Form von zwei Plenarkonferenzen pro Jahr organisiert war, eine Tradition, die bis heute so fortgeführt wird. Dadurch und durch die Arbeit des damals vierköpfigen Sprecherrats entstanden Kontakte zu den politischen Gremien auf Landesebene der beiden Länder, den Hochschulleitungen sowie Unternehmen, gesellschaftlichen Gruppen und Einrichtungen der Erwachsenenbildung/Weiterbildung. Weitere Ergebnisse der Arbeit der Landesgruppe waren Stellungnahmen zur Situation der wissenschaftlichen Weiterbildung an Hochschulen und dann im Jahr 2002 eine Bestandsaufnahme der Angebote der wissenschaftlichen Weiterbildung aller in den beiden Ländern ansässigen 
Hochschulen sowie die Formulierung von Thesen zur wissenschaftlichen Weiterbildung, die größtenteils auch heute noch Gültigkeit haben (Kalis 2002).

Ein weiteres wichtiges Feld war die Organisation von Fachtagungen zu speziellen Themen der wissenschaftlichen Weiterbildung, die über den Kontext der Landesgruppe hinausgingen, zum Beispiel zum Thema der Qualitätssicherung wissenschaftlicher Weiterbildung oder auch der interaktiven Didaktik als Verbindung von Direkt- und Fernstudium. Übergreifende Themen sind auch heute noch Bestandteil der Landesgruppentreffen, werden aber nicht mehr in Form von Tagungen organisiert, da dies in der Regel durch die jeweiligen Arbeitsgemeinschaften der DGWF (AG-E, AG-F und BAG WiWa) übernommen wird.

Nach dem Jahrtausendwechsel gelang auch die Einbindung der Fachhochschulen in die Arbeit der Landesgruppe. Durch die verstärkten Aktivitäten der Fachhochschulen in der wissenschaftlichen Weiterbildung als auch möglicherweise durch die Umbenennung des Arbeitskreises Universitäre Erwachsenenbildung in Deutsche Gesellschaft für wissenschaftliche Weiterbildung und Fernstudium haben offenbar auch die Fachhochschulen die Landesgruppe als einen Ort des wichtigen fachlichen Austausches für sich gesehen.

Heute kennzeichnet die Landesgruppe eine große Vielfalt der Mitglieder. Der überwiegende Teil der Universitäten, Kunsthochschulen, Hochschulen der angewandten Wissenschaften, bis hin zur Berliner Akademie für weiterbildende Studien e. V., in der die wissenschaftliche Weiterbildung der älteren Erwachsenen beheimatet ist, beteiligt sich an der Arbeit der Landesgruppe. Wichtigstes Anliegen der Mitglieder ist immer noch der vertrauensvolle Austausch. Dies ist vor allem vor dem Hintergrund wichtig, insofern die Arbeits- bzw. Koordinationsstellen für wissenschaftliche Weiterbildung oft nur mit einer Person in der Hochschule besetzt sind und für eine erfolgreiche Arbeit der Austausch mit den Kolleg_innen aus anderen Hochschulen unabdingbar ist.

Die Bedingungen für die wissenschaftliche Weiterbildung an den Mitgliedshochschulen sind höchst unterschiedlich. In Berlin, wo die Hochschulen in der Regel eine Überlast an Studierenden zu bewältigen haben, gilt es die wissenschaftliche Weiterbildung trotzdem noch als Aufgabe der Hochschule sichtbar werden zu lassen. Sie wird in erster Linie in der Form der weiterbildenden Masterstudiengänge wahrgenommen. Daneben gibt es aber auch wissenschaftliche Weiterbildungsmöglichkeiten auf Zertifikatsebene sowie die wissenschaftliche Weiterbildung für ältere Erwachsene. Diese Aktivitäten sind immer limitiert dadurch, dass zunächst die grundständige Lehre abgedeckt werden muss. Wenn auch inzwischen ein Teil der ehemals mit Numerus Clausus belegten Studiengänge wieder zulassungsfrei ist, so ist trotzdem zumindest für die nächsten Jahre damit nur wenig Entlastung für die Lehrenden verbunden, sodass für die wissenschaftliche Weiterbildung nur wenig Kapazität zur Verfügung steht. Es gilt, sich durch den kontinuierlichen Ausbau der wissenschaftlichen Weiterbildung für die Zeit nach dem „Studierendenberg“ (falls 
sie für Berlin jemals kommen sollte) zu rüsten, um sich dann verstärkt den Zielgruppen der wissenschaftlichen Weiterbildung zuzuwenden.

Im Flächenland Brandenburg, in dem die Einwohnerzahl des Landes deutlich unter der der Hauptstadt liegt, gibt es Hochschulen, die oft nicht so große Zuläufe durch Studierende zu verzeichnen haben und damit neue Wege der Studierendenakquise beschreiten müssen. Hier ist oft die wissenschaftliche Weiterbildung in einer besseren Lage ihre Angebote nicht nur als wichtigen Teil der Hochschule zu platzieren, sondern auch das Feld der wissenschaftlichen Weiterbildung als aktives Instrument zur Öffnung der Hochschulen für neue Zielgruppen zu nutzen und die Entwicklung hin zum lebenslangen Lernen zu forcieren. So wird im Land Brandenburg das Thema der Anrechnung von Kompetenzen und der Anerkennung außerhochschulisch erworbener Abschlüsse bereits seit Jahren intensiv verfolgt und praktiziert, um die Zielgruppe beruflich Qualifizierte in die Hochschulen zu integrieren. Aktuell steht das Thema "Duales Studium“ im Fokus der politischen und strategischen Überlegungen im Land Brandenburg. Das „Duale Studium“ ist zwar in seiner Ursprungsform kein Thema der wissenschaftlichen Weiterbildung, wird aber aus der Notwendigkeit der Hochschulöffnung für Lifelong Learners und aufgrund der Erfahrung der wissenschaftlichen Weiterbildung mit diversifizierten Zielgruppen in engem Zusammenhang mit dieser gesehen. Hier zeigt sich der Wandel der wissenschaftlichen Weiterbildung im Sinne ihrer Entgrenzung und ihrer Verzahnung mit den Angeboten der grundständigen Lehre. Generell kann festgehalten werden, dass im Land Brandenburg das Thema "Wissenschaftliche Weiterbildung" auch durch regelmäßige Treffen des Arbeitskreises Weiterbildung im Ministerium für Wissenschaft, Forschung und Kultur gut verankert und intensiv verfolgt wird und dabei auch die DGWF und ihre Arbeit große Beachtung finden.

Als wichtiges, gemeinsames strategisches Ziel wird in der Landesgruppe die kontinuierliche Stärkung der wissenschaftlichen Weiterbildung in den jeweiligen Hochschulen und Universitäten wie auch die Information und Etablierung in Institutionen und Verbänden verstanden. Die unterschiedlichen Ausgangslagen in den beiden Bundesländern sind dabei kein Hindernis, sondern bieten den Akteuren nützliche Einblicke in Vorgehensweisen und Lösungsansätze für unterschiedlichste Problemstellungen.

In den letzten Jahren konnten die Projekte des Förderprogramms „Aufstieg durch Bildung: offene Hochschulen“ in die Arbeit der Landesgruppe eingebunden werden. Alle Projekte haben sich inzwischen vorgestellt und begrüßen den Austausch, der dort möglich ist. Wichtig ist, die Akteure auch nach Auslaufen des Förderprogramms in der Landesgruppe zu halten, da absehbar ist, dass zumindest ein Teil der Projekte verstetigt werden wird und diese im Sinne der oben beschriebenen Entgrenzung auch Teil der wissenschaftlichen Weiterbildung sind.

Eine neue Herausforderung, nicht nur für die Landesgruppe, ist die Integration der Geflüchteten in die Hochschulen, bzw. die Frage, wie die wissenschaftliche Weiterbildung im Besonderen dazu beitragen kann. Diesem Thema hat sich die Landes- 
gruppe in einem Halbjahrestreffen zugewandt und wird es auch zukünftig weiter begleiten.

\section{Literatur}

Kalis, P.-O. (Hrsg.) (2002): Wissenschaftliche Weiterbildung an Hochschulen in Berlin und Brandenburg - Eine Bestandsaufnahme. In: AUE-Informationsdienst Hochschule und Weiterbildung, Sonderheft 2002. 


\section{Landesgruppe Hessen}

Silke Vergara

\section{Die Gründung und die Merkmale der Landesgruppe Hessen}

Seit 2004 besteht ein Verbund hessischer Hochschulen im Bereich der wissenschaftlichen Weiterbildung, das Netzwerk WissWeit. In diesem Verbund wurden für Hessen u.a. Weiterbildungsformate entwickelt, gemeinsame Marketingangebote geschaffen und als zentraler Bereich eine umfangreiche Datenbank von Weiterbildungsangeboten aufgebaut. Die DGWF-Landesgruppe Hessen wurde ausgehend von diesem Verbund im Dezember 2011 in Frankfurt am Main von den hessischen Hochschulen' gegründet.

Die DGWF-Landesgruppe Hessen trifft sich in der Regel einmal jährlich zu einer gemeinsamen Sitzung. Für die Mitglieder besteht die Gelegenheit, das regionale Netzwerk für einen Erfahrungstausch in der Weiterbildung zu nutzen. Die wichtigsten Angebote der DGWF-Landesgruppe Hessen sind der Zugang zu Publikationen und aktuellen Forschungsergebnissen sowie die Möglichkeit zur Teilnahme an verschiedenen Tagungsangeboten. Eine überregionale und europaweite Vernetzung der Fachgesellschaft bietet den Mitgliedern der DGWF-Landesgruppe Hessen weitere Kontaktangebote.

\section{Rechtsgrundlagen der Weiterbildung in Hessen}

In Hessen zählt die Weiterbildung neben der Forschung, Lehre und des Studiums zu den Aufgaben der Hochschulen und ist in $\rrbracket_{3}$ (1) des Hessischen Hochschulgesetzes $(\mathrm{HHG})^{2}$ festgeschrieben. Die Rechtsgrundlagen der Weiterbildung sind in $\mathbb{1} 6$ HHG genannt. Nach \16 (1) HHG sollen Hochschulen Angebote der Weiterbildung entwickeln und anbieten. In $₫ 16$ (2) HHG werden allgemeine Zulassungsvoraussetzungen für die Bewerber/innen der weiterbildenden Masterstudiengänge genannt.

1 Gründungshochschulen waren: Goethe-Universität Frankfurt am Main, Philipps-Universität Marburg, Technische Universität Darmstadt, Justus-Liebig-Universität Gießen, Universität Kassel, Frankfurt University of Applied Sciences, Hochschule Darmstadt, Hochschule Fulda, Hochschule RheinMain und Technische Hochschule Mittelhessen (Stand: Dezember 2011).

2 Hessisches Hochschulgesetz 2015. 
\16 (3) HHG schreibt die Erhebung von kostendeckenden Entgelten für die Weiterbildungsangebote vor und regelt den Umgang mit Mitgliedern der Hochschule, die zusätzliche Aufgaben in der Weiterbildung oder in dualen Studienangeboten übernehmen. Die Finanzierung des wissenschaftlichen Personals wird in $\int_{16} 6$ (4) HHG geregelt und fließt bei der Berechnung der Aufnahmekapazität grundständiger Studiengänge nicht ein, wenn es sich dabei ausschließlich um Entgelte in der Weiterbildung handelt. Bei einem Engagement von Hochschulpersonal in der Weiterbildung wird \70 HHG zur Regelung von Nebentätigkeiten des Personals angewandt. \8 (4) HHG ordnet den Verbleib der Überschüsse in der Hochschule. Eine Novellierung im Jahr 2015 ließ den $\ 16$ HHG unverändert.

\section{Aktueller Stand und Ausblick der Landesgruppe Hessen}

Im Jahr 2013 wurde der Bund-Länder-Wettbewerb „Aufstieg durch Bildung: offene Hochschulen“ gestartet. Die nachstehenden hessischen Hochschulen und Mitglieder der DGWF-Landesgruppe Hessen sind aktiv an diesem Projekt beteiligt:

Einzelprojekte:

- Frankfurt University of Applied Sciences: MainCareer - Offene Hochschule

- Hochschule Fulda: Fulda bildet Lebensbegleitend (FuBiLe)

- Technische Universität Darmstadt: Kontinuum - Wissenschaftliche Weiterbildung an der TU Darmstadt

Verbundprojekte:

- Justus-Liebig-Universität Gießen, Philipps-Universität Marburg \& Technische Hochschule Mittelhessen: WM³ Weiterbildung Mittelhessen

- Universität Kassel, Carl von Ossietzky Universität Oldenburg (Koordinator), Fraunhofer Gesellschaft, FernUniversität in Hagen, Universität Stuttgart; NEXT ENERGY, EWE-Forschungszentrum für Energietechnologie e. V.: mint.online

- Technische Universität Darmstadt, Hochschule Darmstadt, Goethe-Universität Frankfurt am Main, Hochschule Albstadt-Sigmaringen (Koordinator), FriedrichAlexander-Universität Erlangen-Nürnberg, Hochschule Offenburg, Ruhr-Universität Bochum, Universität Passau, Fachhochschule Stralsund: Open $C^{3} S-$ Competence Center for Cyber Security

- Hochschule Darmstadt und Fachhochschule Aschaffenburg: Open e-University

- Technische Universität Darmstadt und Hochschule Weserbergland (Koordinator): Open IT Bachelor und Open IT Master - vom IT-Praktiker zum Bachelor Wirtschaftsinformatik und zum Master IT-Business-Management

Aus diesen genannten Projekten werden zahlreiche Forschungsergebnisse und Erkenntnisse erwartet, von denen auch die Mitglieder profitieren können. Ferner können damit auch Projektergebnisse über die Landesgrenzen einfließen. Der Informationsaustausch mit anderen DGWF-Landesgruppen und weiteren Netzwerken der Weiterbildung soll kontinuierlich verbessert werden, um bestimmte Entwicklungen 
wichtiger Themen effizienter nutzen zu können. Das Netzwerk soll dazu dienen die Verbreitung von Fachwissen und die Verwertbarkeit von Wissen allen Mitgliedern zugänglich zu machen (vgl. Barz 2006, S.40).

Die Zahl der Projekte der hessischen Hochschulen zeigt wiederum das gestiegene Interesse am Thema der Weiterbildung und welche Aufgaben damit zukünftig für die Hochschulen verbunden sein werden. Ein zentraler Punkt ist die Öffnung der Hochschulen für andere Zielgruppen. Eine Ausrichtung auf mehr Heterogenität der Zielgruppen kann zu einer Anpassung der Strukturen und der Weiterbildungsangebote führen. Die Umsetzung der strukturellen Erfordernisse wird ein längerfristiger Prozess sein (vgl. Hanft/Brinkmann 2013, S.13). Bei diesen zukünftigen Aufgaben der Hochschule im Bereich der strategischen Weiterentwicklung können die Mitglieder der DGWF-Landesgruppe Hessen von einem Austausch profitieren. Die Systematisierung der Forschungsergebnisse und eine Überleitung in die Praxis der Weiterbildung wird eine der künftigen Aufgaben sein.

Die DGWF-Landesgruppe Hessen hat sich in den zurückliegenden Landessitzungen auf einige wichtige strategische Themen geeinigt. Die wichtigsten Fragen sind:

- Schaffung von Anreizmodellen für das Engagement in der wissenschaftlichen Weiterbildung,

- Konzeptionelle Strategien und Entwicklungsszenarien für hessenweite Modelle,

- Gemeinsame Marketingaktivitäten.

Aber auch aktuelle hochschulpolitische Entwicklungen finden Eingang in die Diskussionen der DGWF-Landesgruppe Hessen, so wie die Integration von Migrantinnen und Migranten, die mit gezielten Angeboten in der Weiterbildung unterstützt werden können. Die Hochschulen stehen bei diesen Aufgaben noch am Anfang und werden einen intensiven Erfahrungsaustausch der Mitglieder notwendig machen.

Als Schnittstelle kann das Hessische Ministerium für Wissenschaft und Kunst von Bedeutung sein, um grundsätzlich den Kontakt in das Ministerium aufzubauen. Das Ziel ist es, sich bei zukünftigen Gesetzesvorhaben oder Änderungen als DGWF-Landesgruppe Hessen stärker einzubringen, um das Thema Weiterbildung mehr in den Fokus zu rücken.

Die Vertiefungsmöglichkeiten der genannten Themen und Aufgaben sind vielfältig und werden die DGWF-Landesgruppe Hessen in den folgenden Jahren vor politische, strategische, aber auch vor praktische Herausforderungen stellen. Der engagierte Einsatz und die aktive Mitarbeit der Mitglieder werden helfen, offene Fragen $\mathrm{zu}$ beantworten und praxisnahe Lösungen zu finden. 


\section{Literatur}

Barz, H. (2006): Innovation in der Weiterbildung. Was Programmverantwortliche heute wissen müssen. Augsburg. Bund-Länder-Wettbewerb „Aufstieg durch Bildung: offene Hochschulen" http://www.wettbewerb-offene-hochschulen-bmbf.de/

Hessisches Hochschulgesetz (2015): Hessisches Hochschulgesetz vom 30. November 2015.

Hanft, A./Brinkmann, K. (2013): Offene Hochschulen. Die Neuausrichtung der Hochschulen auf Lebenslanges Lernen. Einführung. Lebenslanges Lernen an Hochschulen Strukturelle und organisatorische Voraussetzungen. Münster, S. 13-29.

Hanft, A./Pellert, A./Cendon, E./Wolter, A. (2015): Weiterbildung und Lebenslanges Lernen an Hochschulen. Ergebnisse der wissenschaftlichen Begleitung zur ersten Förderphase der ersten Wettbewerbsrunde des Bund-Länder-Wettbewerbs: „Aufstieg durch Bildung: offene Hochschulen“. Oldenburg. https://de.offene-hochschulen.de/public_ libraries $/ 1$ 


\section{Landesgruppe Nord}

Annekatrin Mordhorst/Sabine Riemer/Anno Stockem

\section{Zur Historie - ein bisschen mehr Geschichte!}

Gegründet wurde die Landesgruppe Nord am 14. März 2007 in Rostock.

Die Mitglieder der Landesgruppe Nord der DGWF (Deutsche Gesellschaft für wissenschaftliche Weiterbildung und Fernstudium) kommen aus den norddeutschen Bundesländern Bremen, Mecklenburg-Vorpommern, Niedersachsen, Hamburg und Schleswig-Holstein. 2016 umfasst die Landesgruppe 34 Hochschulen: In Bremen beteiligen sich vier, in Hamburg sieben, in Mecklenburg-Vorpommern vier, in Niedersachsen 16 und in Schleswig-Holstein drei Hochschulen an der Arbeit der Landesgruppe Nord.

Die Servicestelle Offene Hochschule Niedersachsen als Mitglied bildet eine Schnittstelle zur „Offenen Hochschule“. Die Arbeit der Servicestelle gGmbH zielt darauf, Potenziale wissenschaftlicher (Weiter-)Bildung für Berufstätige zugänglich zu machen. Ein besonderer Fokus liegt dabei auf berufstätigen Studien- und Weiterbildungsinteressierten mit und ohne Abitur. Die Servicestelle ist eine Gesellschaft des Landes Niedersachsen. Sie kooperiert seit 2012 mit vielen relevanten Akteuren und Akteurinnen, die die Öffnung der Hochschulen mitgestalten.

Die Landesgruppe Nord ist aus der „Konferenz Universitärer Zentralstellen (KUZ)“ entstanden. Die KUZ gründete sich Ende der 196oer Jahre als informeller Zusammenschluss der Hochschulen in Niedersachsen, die „universitäre Seminarkurse“ zu ihrem Arbeitsgegenstand hatten. Die Seminarkurse waren eine erste Form der wissenschaftlichen Weiterbildung für Hochschulen in der skandinavischen und auch der britischen Tradition. Mit der Ausweitung des Tätigkeitsprofils über die universitären Seminarkurse hinaus und der Ausweitung der Träger der wissenschaftlichen Weiterbildung in die Fachhochschulen hinein vergrößerte sich das Themenfeld und es erweiterte sich die Teilnahme. Mit der Wiedervereinigung der beiden deutschen Staaten kamen weitere Impulse und Anregungen hinzu, insbesondere in der Fernlehre und dem Fernstudium sowie in der Praxisrelevanz hochschulischer Angebote.

Aus der frühen KUZ kamen wesentliche Impulse auch zur Gründung des Arbeitskreises Universitäre Erwachsenenbildung (AUE), der sich schließlich in die DGWF 
wandelte. Die KUZ beschloss auf ihrer Herbstsitzung in Hannover im Jahre 2006 die Organisationsform $\mathrm{zu}$ ändern und $\mathrm{zu}$ einer Landesgruppe der DGWF mit Geschäftsordnung zu werden. Damit konnte sich dieser Zusammenschluss nun auch formell an der Willensbildung der DGWF beteiligen.

\section{Die Zielsetzung der Landesgruppe Nord}

Die Zielsetzung der Landesgruppe gemäß der Geschäftsordnung ist die Förderung von Studium, Lehre, Forschung und Qualitätssicherung auf dem Gebiet der wissenschaftlichen Weiterbildung.

Die Geschäftsordnung sieht vor, dass die Mitglieder der Landesgruppe insbesondere folgende Ziele in den Vordergrund stellen:

- eine Plattform zu bilden für die Diskussion aller theoretischen und praktischen Dimensionen der Weiterbildung an Hochschulen,

- das allgemeine Verständnis für die Hochschulweiterbildung zu fördern,

- eine Infrastruktur aufzubauen, zu pflegen und weiterzuentwickeln für die institutionsübergreifende Entwicklung, Verbreitung und Qualifizierung von Angeboten der wissenschaftlichen Weiterbildung,

- Forschung und Entwicklung zur Weiterbildung an Hochschulen zu initiieren und zu fördern und sich an entsprechenden Projekten zu beteiligen,

- die Weiterbildung an den Hochschulen Deutschlands auf europäischer und internationaler Ebene gemeinsam zu vertreten und an entsprechenden Projekten teilzunehmen.

Wesentliche Formate sind die wechselseitige Beratung und die Durchführung von Tagungen an den Mitgliedshochschulen. Regelmäßig einmal im Frühjahr treffen sich die Mitglieder der Landesgruppe Nord an einer Hochschule zu einer eintägigen Landesgruppensitzung, um dort aktuelle Themen zu diskutieren, sich zu vernetzen und Erfahrungen auszutauschen.

\section{Themenschwerpunkte in der Landesgruppe Nord}

Seit Bestehen der Landesgruppe stehen immer wieder primär Fragen der Finanzierung der wissenschaftlichen Weiterbildung in Hochschulen, insbesondere Trennungsrechnung sowie steuerrechtliche Fragen, Fragen der Organisation und der praktischen Umsetzung im Vordergrund. Die Themen Organisationsmodelle, Zukunft von wissenschaftlicher Weiterbildung, rechtliche Rahmenbedingungen und Personalausstattung werfen regelmäßig neue oder auch bekannte Fragen auf. Darüber hinaus werden zu ausgewählten Themenschwerpunkten Experten/-innen für Impulsreferate eingeladen. z. B. Offene Hochschule, Marketing, Kooperation und Vernetzung, Nutzung und Rechte von Social Media, Wissenschafts-Zeitvertragsgesetz, Trennungsrechnung. 
Die Mitglieder der Landesgruppe haben auf dem Treffen im Frühjahr 2016 beschlossen, im Jahre 2017 folgende Themen vorrangig zu bearbeiten:

1. Weiterbildungs-Strategien in den Weiterbildungseinrichtungen der Hochschulen

2. Wie kann die Qualität in der Didaktik der Lehrenden in der wissenschaftlichen Weiterbildung gesichert werden?

\section{Herausforderungen in den jeweiligen Bundesländern}

Verlässliche und stabile Strukturen für die Weiterbildungseinrichtungen stehen als Herausforderung an erster Stelle. Diese Verbindlichkeiten der Rahmenbedingungen wissenschaftlicher Weiterbildung an Hochschulen zeigen sich in Organisationsformen, Finanzierung, struktureller Verortung sowie in den personellen Ressourcen. Die existenzielle Unsicherheit vieler Einrichtungen und die Abhängigkeit von Drittmitteln macht planbare Arbeit dort nur in limitierten Zeiträumen möglich; z.B. wenn Fördermittel fließen.

Auffallend ist weiterhin, dass mehrere Einrichtungen, z. B. Kiel, Bremen, Flensburg und Rostock, neben der wissenschaftlichen Weiterbildung auch noch die hochschuldidaktischen Fortbildungen anbieten. Die Hochschule Bremen und die Universität Kiel sind zusätzlich noch verantwortlich für Karriereseminare und Seminare zur Gestaltung der Übergänge von der Universität in die Arbeitswelt.

\section{Gesetzgebung zur wissenschaftlichen Weiterbildung}

Die heterogene Auslegung der Gesetzgebung in der „Verwaltungs“-Praxis der Hochschulen wirkt sich zum Teil sehr problematisch auf die erfolgreiche Organisation von wissenschaftlicher Weiterbildung aus. Als Beispiele sollen hier nur das Wissenschaftszeitvertragsgesetz oder die Trennungsrechnung genannt werden. Zur Vollkosten- und Trennungsrechnung wird die Kultusministerkonferenz Ende 2016/Anfang 2017 eine Empfehlung vorlegen.

$\mathrm{Zu}$ finden sind die Gesetzgebungen zur Organisation der wissenschaftlichen Weiterbildung an Hochschulen für Bremen im Bremischen HSG; \58-60, für Hamburg im Hamburgischen Hochschulgesetz (HmbHG), \57, für Mecklenburg-Vorpommern im Gesetz über die Hochschulen des Landes Mecklenburg-Vorpommern (Landeshochschulgesetz - LHG M-V): $『 31$ und für Schleswig-Holstein im HSG Schleswig-Holstein $\llbracket 58$; 59. In Niedersachsen sieht das Gesetz keine Vorgaben für die Organisation der wissenschaftlichen Weiterbildung vor. 


\section{Good Practice}

Konstruktiv für die langfristig erfolgreiche Entwicklung von wissenschaftlicher Weiterbildung an Hochschulen sind verbindliche Aussagen von Ministerien oder anderen übergeordneten Stellen, wissenschaftliche Weiterbildung als feste Größe an Universitäten und Hochschulen zu betrachten. Im regelmäßigen Dialog mit diesen übergeordneten Einrichtungen gibt es hier auch die Möglichkeit zur Kommunikation über problematische und hinderliche Paragrafen in der Gesetzgebung.

Sehr positiv hat sich die in der Landesgruppe übliche Vorgehensweise erwiesen, bei Fragen alle Mitglieder der Landesgruppe online anzusprechen. Die Resonanz ist sehr hoch und konstruktiv. Zukünftig sollen auch wiederkehrende Fragen über ein Wiki auf den Internetseiten der Landesgruppe Nord kommuniziert werden.

\section{Perspektiven und Pläne der Landesgruppe}

$\mathrm{Zu}$ den bereits genannten Schwerpunktthemen für die nahe Zukunft hatte sich die Landesgruppe Nord bereits 2013 über wichtige Zukunftsthemen und bildungspolitische Ziele verständigt:

1. Die Rahmenbedingungen (personell, organisatorisch, rechtlich und politisch) müssen geklärt und stabilisiert werden.

2. Das Selbstverständnis der Weiterbildung muss „Wirklichkeit“ werden.

3. Die wissenschaftliche Weiterbildung entwickelt sich weiter, und damit muss auch der Fokus auf die Zielgruppen, die Lehr-/Lernformate und die Lehrenden verändert und an die Bedürfnisse beständig angepasst werden.

Wichtig ist auch, den Transfer der Ergebnisse der Projekte der „offenen Hochschule“ dauerhaft sicherzustellen. 


\section{Landesgruppe Nordrhein-Westfalen}

STEFAN GESMAN N

\section{Zur Landesgruppe NRW}

Die Landesgruppe Nordrhein-Westfalen (NRW) wurde nach einer längeren Ruhephase im Herbst 2012 auf Anregung von Bernhard Christmann (ehemaliger Leiter der Arbeitsstelle Wissenschaftliche Weiterbildung an der Ruhr-Universität Bochum) reaktiviert. Im Vordergrund ihrer Arbeit steht die Vernetzung der Mitglieder untereinander, aber auch die Kommunikation und Kooperation mit anderen Akteuren der Weiterbildung in der Region. In den ersten drei Jahren seit Bestehen der Landesgruppe standen primär Fragen der Organisation und Umsetzung von Angeboten der wissenschaftlichen Weiterbildung in Hochschulen im Vordergrund. In unterschiedlichen Arbeitsgruppen wurde u. a. zu den Themen Marketing, Kooperation, Organisationsmodelle, Profil der Hochschule, rechtliche Rahmenbedingungen sowie Finanzierung und steuerrechtliche Fragen gemeinsam gearbeitet. Darüber hinaus konnten $\mathrm{zu}$ ausgewählten Themenschwerpunkten Experten/-innen für Impulsreferate gewonnen werden (z. B. Kooperation und Vernetzung, Führung von unten, CustomerRelationship-Management und didaktische Planung und Gestaltung von BlendedLearning-Angeboten).

Die Landesgruppe NRW trifft sich regelmäßig im Frühjahr zu einer zweitägigen und im Herbst zu einer eintägigen Landesgruppensitzung.

\section{Zu den rechtlichen Rahmenbedingungen der wissenschaftlichen Weiterbildung in NRW}

Wissenschaftliche Weiterbildung gehört neben der Gewinnung wissenschaftlicher Erkenntnisse, anwendungsbezogener Lehre und Studium, Forschungs- und Entwicklungsaufgaben, Förderung des wissenschaftlichen Nachwuchses sowie künstlerisch-gestalterischen Aufgaben zu den Kernaufgaben von Universitäten und Fachhochschulen gemäß $\mathbb{3}_{3}$ Abs.1 und 2 Gesetz über die Hochschulen des Landes Nordrhein-Westfalen (Hochschulgesetz - HG). 
Die Rahmenbedingungen für wissenschaftliche Weiterbildung regelt der $₫ 62 \mathrm{HG}$. Hierbei differenziert der Gesetzgeber grundsätzlich zwischen wissenschaftlicher und künstlerischer Weiterbildung, ohne diese Differenzierung weiter zu konkretisieren. Im Absatz 1 des $₫ 62$ HG werden darüber hinaus zwei Formen der künstlerischen bzw. wissenschaftlichen Weiterbildung unterschieden: die Form des weiterbildenden Studiums und die des weiterbildenden Masterstudiengangs. Während der weiterbildende Masterstudiengang im Absatz 3 des $₫ 62$ HG weitergehend konkretisiert wird („Ein weiterbildender Masterstudiengang ist ein Studiengang, der neben der Qualifikation nach $\ 49$ das besondere Eignungserfordernis eines einschlägigen berufsqualifizierenden Studienabschlusses und das besondere Eignungserfordernis einer einschlägigen Berufserfahrung voraussetzt.“), bleibt das Format des weiterbildenden Studiums weitestgehend unbestimmt. Im Absatz 4 wird lediglich darauf verwiesen, dass die Teilnehmenden des weiterbildenden Studiums Weiterbildungszertifikate erhalten und Näheres über die Prüfungsordnung zu regeln sei. Dies führt in der Praxis der wissenschaftlichen Weiterbildung dazu, dass recht unterschiedliche Umsetzungswege in Bezug auf die Form des weiterbildenden Studiums vorzufinden sind. So lassen sich nicht nur unterschiedliche Bezeichnungen (Zertifikatsweiterbildungen, Zertifikatslehrgänge, Weiterbildende Studien etc.), sondern auch unterschiedliche Zulassungsvoraussetzungen, Abschluss- und Anrechnungsverfahren (ECTS-Punkte) und Umsetzungsmodalitäten konstatieren. Einerseits führt die Unbestimmtheit des Begriffs des weiterbildenden Studiums dazu, dass in NRW ein recht buntes Feld an Angeboten der wissenschaftlichen Weiterbildung vorzufinden ist, andererseits trägt eben jene Unbestimmtheit dazu bei, dass eine eindeutige Abgrenzung gegenüber Dritten (z. B. privaten Weiterbildungsanbietern) erschwert wird.

Dienstrechtlich betrachtet, gehört die wissenschaftliche Weiterbildung zum Hauptamt der Lehrenden. Da jedoch das grundständige Lehrangebot immer Vorrang genießt, wird die Lehre in der wissenschaftlichen Weiterbildung in der Praxis nicht der Lehrverpflichtung angerechnet, sondern i. d. R. zusätzlich vergütet.

Da weiterbildende Masterstudiengänge durch die Teilnehmenden und nicht durch den Landeszuschuss finanziert sind, führt dies nicht zur Erhöhung der Aufnahmekapazitäten. Weiterbildende Studiengänge werden somit nicht in die Kapazitätsberechnung einbezogen.

Es wäre wünschenswert, dass Weiterbildung als Dienstaufgabe grundsätzlich angemessen auf Kapazität und Lehrdeputat angerechnet werden kann. Hierbei gälte es jedoch zugleich zu berücksichtigen, dass Angebote der wissenschaftlichen Weiterbildung zu Vollkosten kalkuliert werden müssen. Das Einbringen des eigenen Lehrdeputats dürfte somit nicht dazu führen, dieses Prinzip der Vollkostenrechnung zu umgehen. 


\section{Zur Zusammenarbeit mit dem Landesministerium}

Die Landesgruppe NRW versteht sich als eine zentrale Schnittstelle zwischen den Hochschulen in NRW und der Landesregierung, hier insbesondere dem Ministerium für Innovation, Wissenschaft und Forschung (MIWF). Aus diesem Grunde sind jährliche Treffen mit den Vertretern/-innen des Ministeriums geplant, um einen konstruktiven fachlichen Austausch über (weiter-)bildungspolitische Themen zwischen der Landesgruppe und dem Ministerium zu ermöglichen.

In diesem Zusammenhang sollen insbesondere Fördermodalitäten für Nutzer/-innen von Angeboten der wissenschaftlichen Weiterbildung mit den Vertretern/-innen des Ministeriums thematisiert werden. Während in der Vergangenheit mit dem Bildungsscheck NRW ein Förderinstrument für Berufstätige in NRW zur Verfügung stand, das auch von vielen Teilnehmer/-innen von Angeboten der wissenschaftlichen Weiterbildung genutzt wurde, haben Veränderungen in den Zugangsvoraussetzungen für den Bildungsscheck in den vergangenen Jahren dazu geführt, dass kaum noch Teilnehmende von Angeboten der wissenschaftlichen Weiterbildung dieses Instrument einsetzen können (Begrenzung auf Berufstätige mit einem zu versteuernden Jahreseinkommen von max. $30.000 €$ bzw. 60.000€ bei gemeinsamer Veranlagung; Zuspitzung der Zielgruppe auf Zugewanderte bzw. Menschen mit Migrationshintergrund, Berufsrückkehrende, Beschäftigte ohne Berufsabschluss, Un- oder Angelernte, Ältere ab 50 Jahren sowie atypische Beschäftigungsverhältnisse).

Darüber hinaus soll mit den Vertretern/-innen des Ministeriums erörtert werden, inwiefern Angebote der wissenschaftlichen Weiterbildung auch im Zuge des Gesetzes zur Freistellung von Arbeitnehmern zum Zwecke der beruflichen und politischen Weiterbildung - Arbeitnehmerweiterbildungsgesetz (AWbG) - Berücksichtigung finden könnten. Gemäß dem AWbG haben Arbeiter und Angestellte, deren Beschäftigungsverhältnisse ihren Schwerpunkt in NRW haben, einen Anspruch auf Arbeitnehmerweiterbildung im Umfang von fünf Tagen pro Jahr. Damit auch Weiterbildungsangebote von Hochschulen im Sinne des AWbG Anerkennung finden könnten, müssten Hochschulen gemäß der Bestimmungen des Weiterbildungsgesetzes NRW (WbG) von der jeweils zuständigen Bezirksregierung als anerkannte Einrichtung der Weiterbildung bewertet werden (die Voraussetzungen für eine solche Anerkennung werden im $\sqrt{15} \mathrm{WbG}$ geregelt). Hier gilt es gemeinsam mit den Vertretern/-innen des Ministeriums zu diskutieren, inwiefern eine solche Anerkennung unter Berücksichtigung bereits vorhandener Akkreditierungen möglich wäre, ohne dass hiermit zugleich zwingend ein Anspruch auf finanzielle Förderung durch das Land NRW verbunden wäre $(\mathbb{1} 16, \mathrm{WbG})$. 


\section{Good-Practice-Beispiele aus der Landesgruppe}

Die Landesgruppe NRW hat in ihren letzten Arbeitstreffen zahlreiche Good-PracticeBeispiele zusammengetragen, die jeweils von ihrer zentralen Verortung innerhalb der Hochschule, ihrem davon ausgehenden Bildungsauftrag (fachübergreifend oder fachspezifische Ausrichtung) und ihren zur Verfügung stehenden Ressourcen abhängen. Dazu gehören beispielsweise:

- Transdisziplinäre und fakultätsübergreifende Weiterbildungsprogramme unter Beteiligung mehrerer Fakultäten zu interdisziplinären Themen

- Erwerb des deutschen Weiterbildungspreises für eine Maßnahme

- Entwicklung von differenzierten Marketingstrategien, die mehrere Vertriebskanäle berücksichtigen sowie auf die jeweiligen Zielgruppen zugeschnitten sind

- Entwicklung und Realisierung kompetenz- und transferorientierter BlendedLearning-Formate

- Modularisierung von weiterbildenden Masterstudiengängen, um unterschiedlichen Weiterbildungsbedürfnissen und Lebenssituationen Berufstätiger gerecht zu werden

- zielgerichtete Bedarfserhebungen zur (Weiter-)Entwicklung von Weiterbildungsprogrammen

- Bündelung regionaler Synergien zur Realisierung von Weiterbildungsprogrammen (Kooperationen zwischen Hochschulen, teilweise auch mit externen Bildungsanbietern)

- Qualitätsmanagement für den Bereich Wissenschaftliche Weiterbildung durch Zertifizierung bzw. differenziertes Prozessmanagement.

\section{Aufstieg durch Bildung: Offene Hochschule}

Ein Mitglied der Landesgruppe, die Hochschule Niederrhein, erhält für das Projekt „Wissenschaftliche Weiterbildung und Wissenstransfer für die Region“ eine Förderung aus dem BMBF-Programm „Aufstieg durch Bildung“. Mit diesem Projekt möchte die Hochschule Niederrhein einen Beitrag zur Fachkräftesicherung in der Region leisten, indem sie ein Weiterbildungsangebot auf Masterniveau für die Region Niederrhein systematisch neu entwickelt. Grundbausteine der wissenschaftlichen Weiterbildung sind Hochschulzertifikate.

Beim zweitägigen Frühjahrstreffen, das im April 2016 an der Hochschule Düsseldorf unter dem Thema „Kundenbeziehungsmanagement“ stand, haben zwei Mitglieder des Projektteams die Ergebnisse einer großflächigen Unternehmensbefragung vorgestellt, die im Rahmen des Projekts durchgeführt wurde.

Ein Kontakt zu den regionalen Netzwerken der Offenen Hochschule besteht indirekt über die Projektleiterin des BMBF-Projektes der Hochschule Niederrhein, die an Veranstaltungen teilnimmt, die von diesen drei Netzwerken organisiert werden. 


\section{Wohin die Reise geht: Perspektiven und Pläne der Landesgruppe NRW}

Die Landesgruppe NRW setzt sich das Ziel, den Auf- und Ausbau von Angeboten der wissenschaftlichen Weiterbildung an Hochschulen in NRW zu forcieren. Zu diesem Zweck plant die Landesgruppe NRW zukünftig verstärkt eine beratende Funktion in Bezug auf solche Hochschulen in NRW einzunehmen, die sich erstmalig oder erneut auf den Weg machen wollen, um Angebote der wissenschaftlichen Weiterbildung an der eigenen Hochschule zu verankern. Hierbei kann die Landesgruppe sowohl auf die hohe fachliche Expertise der Landesgruppenmitglieder als auch auf die bis dato bereits erarbeiteten Arbeitsergebnisse der Landesgruppe zurückgreifen.

Um adäquate Rahmenbedingungen für die Umsetzung von Angeboten der wissenschaftlichen Weiterbildung an Hochschulen in NRW gewährleisten zu können, plant die Landesgruppe NRW zukünftig verstärkt den Kontakt zur Landesregierung aufzunehmen. So sind jährlich stattfindende Gespräche mit den Vertretern/-innen des MIWF geplant, auch um u. a. die bereits genannten rechtlichen Hürden zur Umsetzung von Angeboten der wissenschaftlichen Weiterbildung an Hochschulen in NRW gemeinsam zu diskutieren.

Darüber hinaus wird die Landesgruppe NRW auch weiterhin Kooperations- und Dialogräume für ihre Mitglieder schaffen und großen Wert auf das „Lernen von anderen" und Sammeln von Good Practices legen. Zu diesem Zwecke werden bei den jährlichen Treffen aktuelle Fragen diskutiert und Fallbeispiele besprochen sowie ausgewählte Experten/-innen eingeladen. 



\title{
Landesgruppe Mitteldeutschland
}

\author{
Maria Kondratjuk/Kerstin Tänzer
}

Mit der Gründung der Landesgruppe Mitteldeutschland im April 2014 wurde ein lange gehegter Vernetzungsgedanke umgesetzt. Seither verfolgt die Landesgruppe Mitteldeutschland das Ziel, die Weiterbildungsakteure der Hochschulen und hochschulnahen Einrichtungen der wissenschaftlichen Weiterbildung aus den drei Bundesländern Sachsen, Sachsen-Anhalt und Thüringen (noch) enger zu vernetzen. Diese Vernetzung unter dem Dach der DGWF soll eine hochschul- und länderübergreifende Zusammenarbeit und einen thematisch fokussierten Austausch ermöglichen, um damit gemeinsame Schnittstellen, Herausforderungen und Themen zu bearbeiten. Die dadurch entstehenden Synergieeffekte fließen zum einen in die tägliche Arbeit ein und führen zum anderen zu neuen Fragestellungen und Themenschwerpunkten (vgl. Tänzer 2014).

Der kollegiale Austausch und die Auseinandersetzung mit relevanten Themenstellungen werden von allen Mitgliedern und Gästen der Landesgruppe aktiv genutzt. So finden seit dem Gründungstreffen regelmäßig im Frühjahr und im Herbst die Landesgruppentreffen statt. Als Gastgeber fungiert dabei jeweils eine Mitgliedshochschule abwechselnd aus einem der drei Bundesländer. Neben einem Tätigkeitsbericht des Sprecherrates zählen die Berichte aus dem DGWF-Vorstand und die Neuigkeiten aus den Ländern, wie z.B. neue Landesgesetzgebungen, mittlerweile zu festen Programmpunkten. In der Rubrik „Mitteilungen aus den Hochschulen“ erhalten alle Anwesenden die Möglichkeit, von ihren aktuellen Arbeitsschwerpunkten, Projektergebnissen und besonderen Herausforderungen in der wissenschaftlichen Weiterbildung zu berichten. Somit wird stets ein recht umfassendes Bild der Weiterbildungsaktivitäten aller in der Landesgruppe Mitteldeutschland engagierten Hochschulen und Einrichtungen der wissenschaftlichen Weiterbildung gezeichnet.

Für die inhaltliche Landesgruppenarbeit wurden erstmals beim Gründungstreffen einige für alle Beteiligten wichtige Themen identifiziert, zu denen sich die Gruppe austauschen will bzw. die bearbeitet werden sollen. Diese sind z. B.: Organisationsformen, Geschäftsmodelle (z. B. Thema Auslagerung); die Entwicklung und Implementation von berufsbegleitenden Studiengängen (z. B. organisatorischer Aufwand); Institutionalisierung (z. B. Projekt vs. Haushalt); Dialog mit der Wirtschaft sowie Wissens- und Technologietransfer; Stellenwert der Hochschulweiterbildung; Gebüh- 
ren- und Weiterbildungsordnung; die Bewältigung von Ressourcenproblemen (z. B. Umgang mit gesamthochschulischen Kürzungen); Marketing und Markterschließung; besondere Formate und Programme (berufsbegleitend, Studieren ab 50); Verknüpfung mit den Debatten in der Hochschuldidaktik und den Schlüsselkompetenzen (z. B. Heterogenität); Kooperationen (z. B. hochschulübergreifend).

Diese Themenliste wird regelmäßig aktualisiert und entsprechend der Bedürfnisse der Mitglieder und Gäste der Landesgruppe erweitert. Im Fokus steht die Verknüpfung von Wissenschaft und Praxis durch Weiterbildung. Denn „[w]issenschaftliche Weiterbildung ist keine einfache Brücke, welche die ,Lücke' zwischen Praxis und Wissenschaft elegant schließen könnte. Sie ist vielmehr ein voraussetzungsreicher und kontinuierlicher Prozess der sorgfältigen Analyse von weiterbildungsrelevanten Entwicklungen in Praxis und Wissenschaft und deren Vermittlung zu forschungsgeleiteter Wissensproduktion" (DGWF-Empfehlungen 2015, S. 7). Einzelne Themen werden dabei vor allem direkt bei den Treffen mit Impulsbeiträgen sowie Praxisbeispielen näher beleuchtet und daran anschließende Fragestellungen in kleinen Teams erörtert und präsentiert. Hierzu gehörten zuallererst die Finanzierung von Weiterbildung im Kontext von Vollkosten- und Trennungsrechnung sowie die Anrechnung von außerhochschulisch erworbenen Kompetenzen. Dabei diente jeweils ein Best-Practice-Beispiel einer Hochschule als Ausgangspunkt für Diskussion und Austausch zur Umsetzung in der Praxis an den einzelnen Hochschulen. So konnte Manuela Koch-Rogge von der Hochschule Harz gewonnen werden, die ein Modell zur Anrechnung bereits erworbener Kompetenzen vorstellte, besonderer Fokus lag dabei auf konzeptionellen Vorarbeiten (vgl. dazu Koch/Westermann 2006; Koch-Rogge 2016).

Unter der Überschrift „Entwicklung der Hochschullandschaft“ wurden die Hochschulentwicklungsplanung in den drei Ländern sowie die strategische Ausrichtung der wissenschaftlichen Weiterbildung an den einzelnen Hochschulen betrachtet und verglichen. In den drei Bundesländern ist die Weiterbildung recht unterschiedlich vom Umfang und der Ausrichtung in den jeweiligen Hochschulentwicklungsplänen verankert. Laut einer landesgruppeninternen Umfrage ${ }^{1}$ ist die Hochschulweiterbildung grundsätzlich zu wenig in den Plänen repräsentiert. Die zum Teil sehr unterschiedlichen strukturellen Verankerungen und finanziellen sowie personellen Ausstattungen zeigen die Dynamik im Feld und sind Marker für die noch anhaltende Reorganisation der wissenschaftlichen Weiterbildung (vgl. dazu Dollhausen/ Ludwig/Wolter 2013). Die Landesgruppe Mitteldeutschland wird sich daher noch stärker mit der Verortung der Hochschulweiterbildung als profilbildendem ${ }^{2}$ Element als Gegenstand der Hochschulentwicklung beschäftigen. Dabei können zunächst allgemeine Aspekte der Hochschulentwicklung betrachtet werden, wie die Verände-

1 Der Sprecherrat der Landesgruppe Mitteldeutschland hat eine landesgruppeninterne Umfrage zum Verständnis und der Auslegung der hochschulischen Weiterbildung an den einzelnen Hochschulen initiiert, um ausgehend von dieser Bestandsaufnahme Implikationen für die weitere Arbeit zu formulieren. Hierzu gibt es ein internes Arbeitspapier, welches bei fortgeschrittener Bearbeitung publiziert wird.

2 Wenngleich zwischen vertikaler (Reputation, Qualität u. a.) und horizontaler (Regionalität, Praxisbezug u. a.) Profilbildung unterschieden werden sollte (vgl. dazu Teichler 1999). 
rungen in der Hochschullandschaft und die Hochschule als Organisation mit ihren besonderen strukturellen Merkmalen. Bei einer konkreteren Betrachtung, die sich auf die jeweilige Institution bezieht, kann wissenschaftliche Weiterbildung als Wissenschaftsmanagement diskutiert werden, Hochschulentwicklungs- und Hochschulstrukturpläne sowie die Positionierung von Weiterbildung noch präziser in den Blick genommen und sich letztlich mit hochschulpolitischen Implikationen auseinandergesetzt werden. Die sich daraus ergebenden Spannungsfelder (z. B. Bildungsauftrag vs. Kostendeckung, Projektförmigkeit vs. Nachhaltigkeit, Positionierung vs. Profilierung) können gemeinsam diskutiert werden. Denn auch wenn z. B. die Tätigkeiten in der Hochschulweiterbildung durch Zielvereinbarungen, Leitbilder und Tätigkeitsbeschreibungen formal definiert werden, stellt sich die allägliche Arbeitsrealität jedoch mit anderen Regeln, Dringlichkeiten und Prämissen dar (vgl. Kondratjuk/ Schulze 2014).

Im Zuge dieser Auseinandersetzung entstand die Idee, ein Leitbild der Landesgruppe Mitteldeutschland zu erarbeiten, welches das gemeinsame Verständnis aller beteiligten Hochschulen und deren ausführenden Akteuren über wissenschaftliche Weiterbildung widerspiegelt. Darauf aufbauend ist langfristig die Erarbeitung eines Positionspapiers geplant, in dem die Weiterbildung verstärkt in den Fokus der Hochschulentwicklung gerückt wird. Das Positionspapier soll dabei als Grundlage für den hochschulinternen Dialog dienen. Strategisch hat sich die Landesgruppe Mitteldeutschland aber auch zum Ziel gesetzt, sich aktiv mit Empfehlungen und Stellungnahmen in den länderbezogenen Diskussionsprozess mit Politik und Gesellschaft einzubringen. Ein Positionspapier kann hierfür der Ausgangspunkt für hochschulpolitische Aktivitäten der Landesgruppe sein. Es besteht zudem das Bestreben, auch die Landesministerien und die Landesrektorenkonferenzen in die Arbeit mit einzubeziehen. Damit wäre es der Landesgruppe Mitteldeutschland möglich, sich durch aktive Mitarbeit bei strategischen Planungen zur Hochschulweiterbildung einzubringen.

Zentral für die Landesgruppe Mitteldeutschland ist der auf Zusammenarbeit und Kooperation basierende Austausch. Regelmäßig erfolgt dies im Rahmen der Landesgruppenarbeit, darüber hinaus bestehen auf Länderebene mitunter langfristig gewachsene Kooperationen zwischen den Hochschulen wie z. B. in bestimmten Formen der Zusammenarbeit, die durch ein Verbundprojekt ${ }^{3}$ zur wissenschaftlichen Weiterbildung aller Hochschulen in Sachsen-Anhalt entstanden sind. Ein Produkt aus dieser Projektlaufzeit ist das Weiterbildungsportal der Hochschulen in SachsenAnhalt (vgl. dazu www.wiweiter.de). Aktive Netzwerkarbeit bildet die Grundlage für nachhaltige Strukturen der Zusammenarbeit. So nehmen an den Landesgruppentreffen Vertreterinnen und Vertreter aus Projekten der Programminitiative „Aufstieg durch Bildung: Offene Hochschulen" teil und bringen ihre Projektansätze, Erfahrungen und Ergebnisse aktiv in die Diskussion ein. Es bestehen zudem Kontakte zur

3 Weitere Informationen dazu unter: http://www.ms.sachsen-anhalt.de/themen/arbeit/fachkraeftesicherung-weiterbil dung/wissenschaftliche-weiterbildung/transferzentren/ 
Koordination des „Expertennetzwerks Mitte“ in Thüringen. Eine mögliche Zusammenarbeit von Expertennetzwerk und Landesgruppe Mitteldeutschland wird sehr begrüßt und wurde bereits angegangen.

In der Zusammensetzung der Landesgruppe wurde großer Wert auf die Vertretung aller drei Bundesländer sowie der unterschiedlichen Hochschultypen gelegt. So ist Yvonne Weigert, Sachgebietsleiterin der Wissenschaftlichen Weiterbildung und Fernstudium an der Universität Leipzig (Sachsen) und Mitglied des Sprecherrates in der BAG WiWA Sprecherin der Landesgruppe Mitteldeutschland. Unterstützt wird Frau Weigert von Kerstin Tänzer, wissenschaftliche Mitarbeiterin im Zentrum für wissenschaftliche Weiterbildung der Hochschule Magdeburg-Stendal (Sachsen-Anhalt), Dr. Brit Arnold, Leiterin des Zentrums für Weiterbildung an der Fachhochschule Erfurt (Thüringen, vgl. Arnold 2016) und Maria Kondratjuk, wissenschaftliche Mitarbeiterin im Projekt im Rahmen des "Qualitätspakt Lehre“ an der Otto-vonGuericke-Universität Magdeburg (Sachsen-Anhalt) und Mitglied im Sprecherrat der AG-Forschung in der DGWF. Nicht nur die regionale Verankerung, sondern auch die Expertise der Akteurinnen spielt eine besondere Rolle. Zusätzliche Schnittstellen zur AG Forschung, der BAG WiWA, der AG-E und AG-F sowie den anderen Landesgruppen in der DGWF und zur Zeitschrift Hochschule und Weiterbildung (ZHWB) können so optimal genutzt werden.

Die Mitglieder der Landesgruppe sind heterogen, was sich in der Vielfalt an Einrichtungen für die Weiterbildung an Hochschulen und deren Angebotspalette und unterschiedlichen Organisationsformen und institutionellen Verankerungen widerspiegelt. $\mathrm{Zu}$ den institutionellen Mitgliedern der Landesgruppe zählen die folgenden Hochschulen

aus Sachsen: Technische Universität Chemnitz, Universität Dresden, Universität Leipzig, Hochschule Mittweida, Westsächsische Hochschule Zwickau;

aus Sachsen-Anhalt: Martin-Luther-Universität Halle-Wittenberg, Otto-von-GuerickeUniversität Magdeburg, Hochschule Magdeburg-Stendal, Hochschule Merseburg und

aus Thüringen: Fachhochschule Erfurt, Technische Universität Ilmenau, Ernst-AbbeFachhochschule Jena, Bauhaus Universität Weimar.

Außerdem sind einige persönliche Mitglieder in der Landesgruppe aktiv. Als Gäste nehmen regelmäßig Vertreterinnen und Vertreter folgender Hochschulen und Einrichtungen teil: Hochschule Anhalt, Hochschule Harz, HTWK Leipzig, Fachhochschule Schmalkalden, Bauhaus Weiterbildungsakademie, Jenaer Akademie für Lebenslanges Lernen e. V., TU Bergakademie Freiberg u.a.

Die Besonderheit der Landesgruppe Mitteldeutschland liegt jedoch nicht nur in der Repräsentation der drei Bundesländer Sachsen, Sachsen-Anhalt und Thüringen, sondern auch in der synoptischen und synergetischen Zusammenführung gemeinsamer Themen wie z. B. die Auslegung der Landeshochschulgesetze in Bezug auf 
die Hochschulweiterbildung (siehe Abbildung 1), deren Organisationsformen sowie die Ausrichtung der Gebühren- und Entgeltordnungen.

\begin{tabular}{|c|c|c|}
\hline Sachsen & Sachsen-Anhalt & Thüringen \\
\hline $\begin{array}{l}\text { Quelle: Sächsisches Hochschulfrei- } \\
\text { heitsgesetz vom 15. Januar 2013, in } \\
\text { der Fassung vom 01.01.2015: } \\
\text { \338 Weiterbildende Studien: (2) } \\
\text { Weiterbildende Studiengänge setzen } \\
\text { einen ersten berufsqualifizierenden } \\
\text { Hochschulabschluss voraus und } \\
\text { führen nach Maßgabe verbindlicher } \\
\text { Studien- und Prüfungsordnungen } \\
\text { zu einem weiteren berufsqualifizie- } \\
\text { renden Abschluss. Weiterbildende } \\
\text { Masterstudiengänge setzen eine be- } \\
\text { rufspraktische Erfahrung von in der } \\
\text { Regel nicht unter einem Jahr voraus. } \\
\text { Weiterbildende Studiengänge kön- } \\
\text { nen auch als Fernstudiengänge an- } \\
\text { geboten werden. }\end{array}$ & $\begin{array}{l}\text { Quelle: Hochschulgesetz des } \\
\text { Landes Sachsen-Anhalt (HSG LSA) } \\
\text { vom 14. Dezember 2010: } \\
\text { 『 } 16 \text { (1) Die Hochschulen entwickeln } \\
\text { und bieten Möglichkeiten der Wei- } \\
\text { terbildung an, die der wissenschaft- } \\
\text { lichen Vertiefung und Ergänzung be- } \\
\text { rufspraktischer Erfahrungen dienen. } \\
\text {...Die Veranstaltungen sind mit dem } \\
\text { übrigen Lehrangebot abzustimmen. } \\
\text { Berufspraktische Erfahrungen sind } \\
\text { für die Lehre nutzbar zu machen. } \\
\text { Das Weiterbildungsangebot soll aus } \\
\text { in sich geschlossenen Abschnitten } \\
\text { bestehen und die aus der beruflichen } \\
\text { Praxis entstandenen Bedürfnisse } \\
\text { der Teilnehmenden berücksichtigen. } \\
\text { (2) Weiterbildung kann in eigenen } \\
\text { Studiengängen oder einzelnen Stu- } \\
\text { dieneinheiten angeboten werden. } \\
\text { Weiterbildende Studiengänge können } \\
\text { mit einem Hochschulgrad oder einem } \\
\text { Zertifikat abgeschlossen werden. }\end{array}$ & $\begin{array}{l}\text { Quelle: Thüringer Hochschulgesetz } \\
\text { (ThürHG) vom 21. Dezember 2006: } \\
\text { 『51 Die Hochschulen bieten im } \\
\text { Rahmen ihrer Aufgaben Möglichkei- } \\
\text { ten des weiterbildenden Studiums } \\
\text { an. Dabei können sie auch mit } \\
\text { anderen Einrichtungen der Weiter- } \\
\text { bildung außerhalb des Hochschul- } \\
\text { bereichs auf privatrechtlicher } \\
\text { Grundlage zusammenarbeiten. } \\
\text { Die Hochschulen können das weiter- } \\
\text { bildende Studium auch auf privat- } \\
\text { rechtlicher Grundlage anbieten. Die } \\
\text { Durchführung von Lehrveranstaltun- } \\
\text { gen im Rahmen eines weiterbilden- } \\
\text { den Studiums, das in Kooperation } \\
\text { gemeinsam mit einer Einrichtung } \\
\text { der Weiterbildung außerhalb des } \\
\text { Hochschulbereichs durchgeführt } \\
\text { wird, gehört in der Regel nicht zu } \\
\text { den Dienstaufgaben des Personals } \\
\text { mit Lehraufgaben der Hochschule. }\end{array}$ \\
\hline
\end{tabular}

Abb. 1: Vergleich der Landeshochschulgesetze in Mitteldeutschland

Ausgehend von einer Umfrage zu den vorhandenen inhaltlichen und strukturellen Rahmenbedingungen der wissenschaftlichen Weiterbildung an jeder der in der Landesgruppe aktiven Hochschulen und Einrichtungen wurde beim Frühjahrstreffen (April 2016) eine erste Zusammenfassung zu Wirkung, Formaten, Zielgruppen, Gestaltung und Möglichkeiten einer institutionellen Verankerung der wissenschaftlichen Weiterbildung an den Hochschulen als Arbeitsgrundlage vorgestellt. Daraus wurden Implikationen für die Ausrichtung eines von allen Beteiligten gelebten Leitbildes erarbeitet. Eine Arbeitsgruppe übernimmt die Präzisierung dieses Impulspaketes und die Erstellung einer ersten Fassung eines Positionspapiers der Landesgruppe. Bei der Formulierung eines Leitbildes der wissenschaftlichen Weiterbildung in Mitteldeutschland sind u. a. die Berücksichtigung der Hochschule als Ort lebenslangen Lernens, die Umsetzung des Konzepts Offene Hochschule (einschließlich der Gestaltung von Übergängen), die Etablierung der wissenschaftlichen Weiterbildung als Kernaufgabe und als Standortfaktor für die Region (vgl. Kondratjuk 2014) sowie die Fokussierung auf berufsbegleitende Angebote (unter der Prämisse Vereinbarkeit von Familie, Beruf und Weiterbildung) von besonderem Interesse. 


\section{Literatur}

Arnold, B. (2016): 15 Jahre Weiterbildung an der FH Erfurt. In: Hochschule und Weiterbildung, 2016(1), S. 67-69.

DGWF (2015): Deutsche Gesellschaft für wissenschaftliche Weiterbildung und Fernstudium: Organisation der wissenschaftlichen Weiterbildung an Hochschulen. DGWFEmpfehlungen, Oestrich-Winkel (Juli).

Dollhausen, K./Ludwig, J./Wolter, A. (2013): Organisation und Re-Organisation wissenschaftlicher Weiterbildung in einer bewegten Hochschullandschaft. In: Hochschule und Weiterbildung, 2013(2), S.10-13.

Hochschulgesetz des Landes Sachsen-Anhalt (HSG LSA) vom 14. Dezember 2010: http:// www.landesrecht.sachsen-anhalt.de/jportal/?quelle=jlink\&query=HSchulG+ST\&psml= bssahprod.psml\&max=true\&aiz=true [Zugriff: 13.10.2016]

Koch, M./Westermann, G. (2006): Von Kompetenz zu Credits. Anrechnung beruflicher Kompetenzen auf ein Hochschulstudium. Harzer wirtschaftswissenschaftliche Schriften. Deutscher Universitätsverlag.

Koch-Rogge, M. (2016): Studienabbruch und Ausbildungsverkürzung - eine Untersuchung zur Deckungsgleichheit von Studiengängen und Ausbildungsberufen. Präsentation. DOI: 10.13140/RG.2.2.30360.39688 [Zugriff: 14.10.2016]

Kondratjuk, M. (2014b): The University as a local actor for regional adult education? A German statement based on an empirical research. In: Guimaraes, P./Cavaco, C./ Marrocos, L./Paulos, C./Bruno, A./Rodrigues, S./Marques, M. (Hrsg.): Local Change, Social Actions and Adult Learning: Challenges and Responses, Proceedings. Lisbon, S. 122-131.

Kondratjuk, M./Schulze, M. (2014): Forschungsperspektiven auf die Praxis von Hochschulweiterbildung: Ein Blick auf Akteure und die Programmorganisation. In: Hochschule und Weiterbildung, 2014(1), S. 59-64.

Sächsisches Hochschulfreiheitsgesetz vom 15. Januar 2013, in der Fassung vom 01.01.2015. http://www.recht.sachsen.de/vorschrift/10562-Saechsisches_Hochschulfrei heitsgesetz [Zugriff: 13.10.2016]

Tänzer, K. (2014): Gründung der Landesgruppe Mitteldeutschland in der DGWF. In: Hochschule und Weiterbildung, 2014(1), S. $91 \mathrm{f}$.

Teichler, U. (1999): Profilierungspfade der Hochschulen im internationalen Vergleich. In: Olbertz, J.-H./Pasternack, P. (Hrsg.): Profilbildung, Standards, Selbststeuerung. Ein Dialog zwischen Hochschulforschung und Reformpraxis. Weinheim. Deutscher Studienverlag, S. 27-38.

Thüringer Hochschulgesetz (ThürHG) vom 21. Dezember 2006: http://landesrecht.thue ringen.de/jportal/?quelle=jlink\&query=HSchulG+TH\&psml=bsthueprod.psml\&max= true [Zugriff: 13.10.2016] 


\title{
Landesgruppe Rheinland-Pfalz und Saarland
}

\author{
MARGOT KLINKNER
}

\section{Der Weg zur DGWF-Landesgruppe Rheinland-Pfalz und Saarland}

Die Gründung der DGWF-Landesgruppe Rheinland-Pfalz und Saarland im Jahr 2013 geht auf die Initiative der amtierenden Bundesvorsitzenden Dr. Beate Hörr zurück. Als Leiterin des ZWW - Zentrum für wissenschaftliche Weiterbildung an der JGU Mainz hatte Hörr im Jahr 2012 zunächst erwogen, in Ermangelung einer eigenen Regionalgruppe in Rheinland-Pfalz sich der Landesgruppe Hessen anzuschließen. Als daraufhin auch die ZFH - Zentralstelle für Fernstudien an Fachhochschulen in Koblenz auf der DGWF-Mitgliederversammlung im September 2012 ihren Anschluss an die hessische Landesgruppe signalisierte, war dies für Hörr Grund genug, dem Bedarf nach Vernetzung im eigenen Bundesland noch einmal genauer nachzugehen.

Ein Blick auf die Entwicklung der wissenschaftlichen Weiterbildung in RheinlandPfalz zeigt in diesem Kontext, dass der Vernetzungsgedanke in Rheinland-Pfalz nicht neu ist. Bereits 1992 bildete sich an der Universität Mainz mit der „Arbeitsgruppe Hochschulen in der Weiterbildung“ ein Netzwerk aus in dieser Zeit an den Hochschulen neu gegründeten Weiterbildungseinrichtungen, welche bis Ende der 1990er Jahre regelmäßig den fachlichen Austausch pflegten. Der Blick zurück zeigt auch, dass wissenschaftliche Weiterbildung und Fernstudium in Rheinland-Pfalz bereits über eine langjährige Tradition und Erfahrungspraxis verfügen: Schließlich besteht das ZFUW - Zentrum für Fernstudium und universitäre Weiterbildung an der Universität Koblenz-Landau seit nunmehr 25 Jahren, dicht gefolgt vom heutigen DISC - Distance and Independent Studies Center an der TU Kaiserslautern.

Wie die neuerliche Bedarfsabfrage im Jahr 2012 zeigte, bestand auch weiterhin genügend Interesse unter den Hochschulen und hochschulnahen Einrichtungen für einen Zusammenschluss - und dies nicht nur in Rheinland-Pfalz, sondern auch im angrenzenden Saarland. Was lag also näher, als unter dem Dach der DGWF eine weitere Regionalgruppe ins Leben zu rufen, und zwar länderübergreifend für beide 
Bundesländer. Ende Januar 2013 folgte die Gründungsversammlung an der JGU Mainz und im April 2013 fand die konstituierende Sitzung an der ZFH in Koblenz statt. Den Vorsitz der Landesgruppe führt seither Dr. Margot Klinkner von der ZFH, die zusammen mit ihrer Stellvertreterin Angelina Müller vom ZelL - Zentrum für lebenslanges Lernen der Universität des Saarlandes sowie Dr. Markus Lermen vom DISC der TU Kaiserslautern und Johannes C. Mayer von der Deutschen Universität für Verwaltungswissenschaften Speyer den Sprecher/innen-Rat bildet. Der Kreis der Hochschulen, die in der Landesgruppe mitwirken, hat sich seit 2013 kontinuierlich erweitert: Neben der Hochschule Koblenz, der Fachhochschule Bingen und der htw saar - Hochschule für Technik und Wirtschaft des Saarlandes wirken auch Hochschulen in privater Trägerschaft wie beispielsweise die PTHV - Philosophisch Theologische Hochschule Vallendar in der Landesgruppe mit.

\section{Besonderheiten in der Zusammensetzung der Landesgruppe}

Bereits seit ihrer Gründung zeichnet sich die DGWF-Landesgruppe Rheinland-Pfalz und Saarland durch eine außerordentliche Vielfalt ganz unterschiedlicher Einrichtungen für wissenschaftliche Weiterbildung aus: Neben zentralen Einrichtungen an den vier staatlichen Universitäten in Rheinland-Pfalz (Kaiserslautern, Koblenz-Landau, Mainz und Trier), der Universität des Saarlandes, der Hochschule Koblenz und der Katholischen Hochschule Mainz gehören mit dem VCRP - Virtueller Campus Rheinland-Pfalz und der ZFH - Zentralstelle für Fernstudien an Fachhochschulen auch landesweite bzw. länderübergreifende Einrichtungen zu den Mitgliedern. Die Deutsche Universität für Verwaltungswissenschaften Speyer nimmt aufgrund ihrer Bund-Länder-Trägerschaft einen Sonderstatus ein und ist mit ihrem speziellen Zuschnitt auf postgraduierte Masterprogramme und Weiterbildungsveranstaltungen zugleich prädestiniert für das Engagement in der Landesgruppe. Aufgrund der Vielseitigkeit der Einrichtungen und ihrer jeweiligen strukturellen Einbindung in die Hochschule bzw. Hochschullandschaft besteht innerhalb der Landesgruppe auch eine große Spannbreite von Organisations- und Angebotsformen wissenschaftlicher Weiterbildung. Das Spektrum der Studienangebote wissenschaftlicher Weiterbildung bildet dabei das gesamte Fächerrepertoire der Hochschulen in Rheinland-Pfalz und im Saarland ab. Es umfasst persönlichkeitsbildende, berufsqualifizierende wie auch allgemeinbildende Studienangebote, wie z. B. im Rahmen des Gasthörer- und Seniorenstudiums. Die Angebotsformen reichen von Einzelveranstaltungen über modularisierte Angebote mit Zertifikatsabschluss bis hin zu berufsbegleitenden Bachelor- und Masterstudiengängen, welche in Präsenzform, im Blended-Learning-Format oder rein webbasiert durchgeführt werden. Aufgrund von drei Fernstudieneinrichtungen (ZFUW, DISC und ZFH) und dem VCRP nimmt der Anteil der BlendedLearning-Formate und webbasierten Angebote innerhalb der wissenschaftlichen Weiterbildung tendenziell weiter $\mathrm{zu}$. 


\section{Besonderheiten in der Rechtsprechung und damit einhergehende Herausforderungen}

Insbesondere das rheinland-pfälzische Hochschulgesetz eröffnet beruflich Qualifizierten sowohl in Bezug auf ein grundständiges als auch im Hinblick auf ein weiterbildendes Studium breite Zugangsmöglichkeiten. Während eine Berufsausbildung mit qualifiziertem Abschluss und anschließender zweijähriger Berufspraxis bereits den Zugang zum Bachelorstudium eröffnet, besteht für beruflich Qualifizierte, die darüber hinaus drei weitere Jahre einschlägige Berufspraxis nachweisen können, die Möglichkeit über eine Eignungsprüfung zum weiterbildenden Masterstudium zugelassen zu werden. Rheinland-Pfalz hat mit dieser Gesetzgebung die rechtlichen Voraussetzungen für die politisch angestrebte Öffnung der Hochschulen für neue Zielgruppen auf breiter Ebene geschaffen. Eine steigende Nachfrage aus dem Kreis der beruflich Qualifizierten belegt den Bedarf. Bisherige Erfahrungen aus den Masterstudiengängen zeigen hierbei vielfach erste positive Ergebnisse. Sie zeigen aber auch die Anforderung aufseiten der Hochschulen, die Eignungsprüfung im Sinne einer passgenauen Äquivalenzprüfung möglichst sorgsam auszugestalten und gleichzeitig ein differenziertes Angebot von Vor- und Brückenkursen bereitzuhalten, um beruflich Qualifizierten den Weg ins Studium sinnvoll und nachhaltig zu ebnen. Auch wenn die Öffnung der Hochschulzugänge politisch auf breiter Ebene gewünscht wird und die hochschulgesetzlichen Grundlagen geschaffen wurden, treten in der Praxis berufsrechtliche Hürden zutage, die sich auch mithilfe bildungspolitischer Initiativen nicht ohne Weiteres auflösen lassen. So darf beispielsweise die Berufsbezeichnung "Ingenieurin“ oder „Ingenieur" nach wie vor nur führen, wer das Studium einer technischen oder naturwissenschaftlichen Fachrichtung mit einer Regelstudiendauer von mindestens sechs theoretischen Studiensemestern an einer deutschen wissenschaftlichen Hochschule oder an einer deutschen Berufsakademie im tertiären Bildungsbereich mit Erfolg abgeschlossen hat. Beruflich Qualifizierten, die ohne Erststudium über die Eignungsprüfung ein ingenieurtechnisches Masterstudium erfolgreich absolvieren, bleibt somit nach aktueller Rechtsprechung das Führen eines Ingenieurtitels verwehrt. Die Politik ist hier weiterhin gefordert, im Sinne gleichwertiger Hochschulzugänge der hochschulrechtlichen Öffnung des Masterstudiums auch eine berufsrechtliche Gleichstellung der Abschlüsse folgen zu lassen.

\section{Aktuelle Entwicklungen und zukünftige Ausrichtung}

Angesichts der zuvor beschriebenen Diversität hinsichtlich ihrer Zusammensetzung hat sich die Landesgruppe in ihrer jüngsten Vergangenheit einem eingehenden Diskurs zum Selbstverständnis und zur Profilbildung unterzogen und ein Positionspapier veröffentlicht. Neben der Interessenvertretung ihrer Mitglieder und dem Ziel, die Rahmenbedingungen und die institutionellen Strukturen der von Hochschulen organisierten bzw. verantworteten Weiterbildung zu stärken, setzt sich die Landes- 
gruppe auch für die Vernetzung von Akteurinnen und Akteuren auf unterschiedlichen Ebenen ein. Mitglieder der Landesgruppe sind beispielsweise in drei Verbundprojekte der BMBF-Ausschreibung "Aufstieg durch Bildung: offene Hochschulen“ aktiv eingebunden:

- „Offene Kompetenzregion Westpfalz II - OKWestII“: Hochschule Kaiserslautern (Koordinator), Technische Universität Kaiserslautern (DISC), VCRP

- „E-Bildung als Exponent individueller und regionaler Entwicklung - EhochB“: Fachhochschule Kaiserslautern (Koordinator), Technische Universität Kaiserslautern, Hochschule Ludwigshafen am Rhein

- „Bildungsangebot im Bereich der Wirtschaftswissenschaften und Wirtschaftsinformatik für Berufstätige, Personen mit Familienpflichten und Studienabbrechern/innen - Work \& Study“: Hochschule Koblenz (Koordinator), Fachhochschule Worms, Hochschule Bonn-Rhein-Sieg, Hochschule für Technik und Wirtschaft des Saarlandes.

Orientiert an der grundlegenden Zielsetzung, die wissenschaftliche Weiterbildung und das Fernstudium in Rheinland-Pfalz und im Saarland zu fördern und unter besonderer Berücksichtigung der landesspezifischen Gegebenheiten und der rechtlichen Rahmenbedingungen weiterzuentwickeln, richtet sich die Landesgruppe mit ihrer aktuellen Arbeit gezielt an die politische Ebene, um bildungspolitische Entwicklungen im Interesse der wissenschaftlichen Weiterbildung und des Fernstudiums aktiv mitzugestalten. Um eine Sensibilisierung für die Herausforderungen und den Handlungsbedarf im Bereich wissenschaftlicher Weiterbildung und des Fernstudiums zu erzielen, hat die Landesgruppe anlässlich der Landtagswahl in Rheinland-Pfalz Wahlprüfsteine an die Parteien formuliert, deren Antworten ihr eine konkrete Ausgangsbasis für Anschlussgespräche auf Fraktionsebene ermöglichen. In einer von der Landesgruppe initiierten zentralen hochschulpolitischen Diskussionsveranstaltung anlässlich des 6 . Deutschen Weiterbildungstages zum Thema "Weiterbildung 4.0-Zukunftspotenzial für die Hochschulen!?“" konnte sie den angestrebten hochschulpolitischen Diskurs bereits vertiefen: Abgeordnete des rheinland-pfälzischen Landtages diskutierten am 29. September 2016 an der Johannes GutenbergUniversität mit Vertreterinnen und Vertretern aus Hochschulen und hochschulnahen Einrichtungen über aktuelle Herausforderungen an Hochschulen angesichts zunehmender Digitalisierung und beleuchteten im Kontext einer sich abzeichnenden strategischen Neuausrichtung der Hochschulen Stellenwert, Chancen und Rahmenbedingungen wissenschaftlicher Weiterbildung.

Neben ihrer politischen Arbeit auf Landesebene engagiert sich die Landesgruppe auch auf Ebene des EU-Parlamentes: Durch Kontaktaufnahme zu MdEP Sabine Verheyen ist es ihr gelungen, einen Initiativbericht zur wissenschaftlichen Weiterbildung (finaler Titel „Academic further and distance education as part of the European lifelong learning strategy") anzustoßen, an dessen Erstellung die Landesgruppe in den kommenden Monaten aktiv mitwirken wird. 


\section{Zusammenarbeit über die Grenzen hinweg}





\title{
Netzwerk für wissenschaftliche Weiterbildung und Personalentwicklung der Universitäten in Österreich: AUCEN
}

Elke Gornik/Monika Kil/Katharina Mallich-Pötz/

Anna Steiger/Christine Stöckler-Penz

\begin{abstract}
In Österreich hat sich seit 20 Jahren ein Netzwerk für die wissenschaftliche Weiterbildung und Personalentwicklung an Universitäten etabliert: das Austrian University Continuing Education and Staff Development Network - kurz AUCEN. In diesem Beitrag werden die Entstehung von AUCEN, die Rahmenbedingungen der Universitätsgesetzgebung für wissenschaftliche Weiterbildung und Personalentwicklung, Projektaktivitäten und aktuelle Herausforderungen ausgeführt.
\end{abstract}

\section{AUCEN - vom Round Table zur institutionellen Vertretung von wissenschaftlicher Weiterbildung und Personalentwicklung an Universitäten}

AUCEN - das Austrian University Continuing Education and Staff Development Network - wurde vor 20 Jahren als zunächst informelle Plattform ins Leben gerufen. Initiiert durch die Einladung des damaligen Vizerektors für Aus- und Weiterbildung an der Universität für Bodenkultur Wien, gründete sich im März 1996 ein Round Table, um mit Kolleginnen und Kollegen anderer Universitäten Erfahrungen auszutauschen und über Weiterentwicklungsmöglichkeiten im Bereich der universitären

1 Vizerektorate wurden vor dem Hintergrund des Universitätsgesetzes 1993 eingerichtet und Weiterbildung als Aufgabe der Universitäten definiert. 
Weiterbildung ${ }^{2}$ und der universitären Personalentwicklung zu diskutieren. Dieser Round Table, an welchem damals eine kleine Gruppe interessierter Universitätsangehöriger teilnahm, entwickelte sich zunehmend zu einer regelmäßig stattfindenden Plattform. Zwei Jahre später, im Jahr 1998, entwickelte sich die Plattform zu einem Netzwerk mit dem noch heute geltenden Namen „AUCEN - Austrian University Continuing Education and Staff Development Network“. Eine gemeinsame Zielsetzung sowie eine Vertretung nach außen in Form eines vom Netzwerk kollegial bestimmten Sprechers/einer Sprecherin wurden bereits damals festgelegt. 2001 differenzierten sich die beiden Fachbereiche „Universitäre Weiterbildung“ und „Universitäre Personalentwicklung“ stärker aus, sodass sich im Rahmen der Netzwerktreffen separate Arbeitsgruppen für die beiden Bereiche etablierten. Die Expertinnen und Experten für beide Fachbereiche finden bis heute in AUCEN ein Forum für den professionellen Austausch gemeinsamer Interessen und Anliegen im Akteursfeld „Universität“.

Im September 2005 wurde beim 19. AUCEN-Meeting im Rahmen einer Gründungsversammlung beschlossen, dass AUCEN künftig als Verein agieren soll. Die seinerzeit einstimmig angenommenen Statuten, die mit der Österreichischen Rektorenkonferenz (der heutigen Österreichischen Universitätenkonferenz, uniko) abgeglichen und von den Mitgliedsuniversitäten im Vorfeld begutachtet wurden, sind bis heute - mit geringfügigen Adaptierungen - gültig. Als Mitglieder können alle österreichischen Universitäten aufgenommen werden. Als erste Sprecherin des Vereins AUCEN wurde Ada Pellert, damals Vizerektorin für Lehre und Weiterbildung an der Donau-Universität Krems, einstimmig gewählt. Darüber hinaus wurde die Einrichtung einer Geschäftsstelle beschlossen, welche die strategischen, inhaltlichen und organisatorischen Agenden bündeln sollte. Bei der Gründungsversammlung wurden folgende inhaltliche Schwerpunkte für AUCEN festgelegt:

- Strategieentwicklung, Professionalisierung und Institutionalisierung von universitärer Weiterbildung und Personalentwicklung an den österreichischen Universitäten,

- Strategische Positionierung des Vereins in der österreichischen Bildungslandschaft und Lobbying,

- Inhaltlicher Austausch der Netzwerkmitglieder und Weiterentwicklung von Professionalisierungsmaßnahmen in der universitären Weiterbildung und Personalentwicklung.

Diese inhaltlichen Leitlinien blieben für AUCEN essenziell und spiegeln sich im Mission Statement ${ }^{3}$ von AUCEN wider.

20 Jahre nach dem ersten Round Table im Jahr 1996 hat sich AUCEN im österreichischen Bildungssystem als institutionalisiertes Netzwerk etabliert, das über die

2 In Österreich wurde/wird für den Bereich der „wissenschaftlichen Weiterbildung“ das Synonym „universitäre Weiterbildung" benutzt, wobei sich letzterer Begriff in der Bezeichnung des Netzwerkes AUCEN niederschlägt.

3 http://www.aucen.ac.at/fileadmin/user_upload/p_aucen/AUCEN_mission_statement_2016.pdf [Zugriff: 16.05.2016] 
österreichischen Grenzen hinweg bekannt und vernetzt ist. AUCEN hat einen „Full Network“-Status in EUCEN, dem European University Continuing Education Network, und ist dort aktiv mitgestaltend tätig. Die Präsidentschaft von 2010 bis 2015 durch Andrea Waxenegger, die Gründungs- und Vorstandsmitglied bei AUCEN war, hat zur verstärkten europäischen Sichtbarkeit österreichischer wissenschaftlicher Weiterbildungsaktivtäten beigetragen. Aktuell sind von 22 österreichischen Universitäten 20 institutionelle Mitglieder bei AUCEN. Dass AUCEN als Netzwerk positiv wahrgenommen wird, zeigt unter anderem der Bericht und die Erwähnung im österreichischen Universitätsbericht 2014 und die Feststellung, dass sich AUCEN als Plattform für Erfahrungs- und Informationsaustausch zu allen Fragen universitärer Weiterbildung etabliert hat (vgl. BMWFW 2014, S. 174 ff.).

Bis heute ist AUCEN in einer Vereinsstruktur organisiert (siehe im Detail dazu Gornik/Steiger 2015), welche einen Vorstand vorsieht, der sich aus je drei Expertinnen/ Experten in den Fachbereichen Weiterbildung und Personalentwicklung zusammensetzt. Je ein Vorstandsmitglied übernimmt die Funktion des Sprechers/der Sprecherin sowie des Vizesprechers/der Vizesprecherin. Pro Bereich stehen darüber hinaus ein oder mehrere Koordinatorinnen/Koordinatoren als inhaltliche Ansprechpartner und Ansprechpartnerinnen zur Verfügung. Inhaltlich wie organisatorisch koordinierend tätig steht eine Geschäftsstelle zur Verfügung, welche an einer der Mitgliedsuniversitäten angesiedelt ist. Um alle Mitgliedsuniversitäten in die Entscheidungsprozesse miteinzubeziehen, wurde eine Plenarversammlung in den Statuten festgelegt, welche mindestens einmal jährlich stattfindet. Jede Universität nominiert und entsendet Delegierte für die Bereiche universitäre Weiterbildung und universitäre Personalentwicklung. Bei den Plenarversammlungen sind pro Universität zwei Delegierte stimmberechtigt und werden somit in die Entscheidungen aktiv eingebunden.

Essenzieller Bestandteil von AUCEN sind die zweimal jährlich an einer der Mitgliedsuniversitäten stattfindenden Netzwerktreffen. Das 40. AUCEN-Meeting im Herbst 2016 fand gleichzeitig zum 20-jährigen Jubiläum von AUCEN statt. Parallel dazu wurde, zum zweiten Mal nach 2005, mit der Deutschen Gesellschaft für wissenschaftliche Weiterbildung und Fernstudium (DGWF) eine gemeinsame Jahrestagung in Wien durchgeführt.

\section{Rahmenbedingungen und Herausforderungen der universitären wissenschaftlichen Weiterbildung in Österreich}

Der Bereich der wissenschaftlichen Weiterbildung und seine Verankerung an den österreichischen Universitäten hat vor allem in den letzten 15 Jahren eine bedeutsame Entwicklung vollzogen. Ausgelöst wurde diese durch mehrere Einflussfaktoren: Dazu zählen internationale Entwicklungen wie der Bologna-Prozess und die damit verbundene Veränderung der österreichischen Studienarchitektur, rechtliche 
Veränderungen (Universitätsgesetz 2002 und die damit verbundene Autonomie der Universitäten), aber auch soziodemografische Entwicklungen (im Sinne des Lifelong Learning und der veränderten Bildungsbedarfe), die Frage nach zusätzlichen Finanzierungsquellen für Universitäten sowie die Frage nach der Profilbildung und Positionierung im nationalen und internationalen Bildungsbereich.

Aktuell wird die wissenschaftliche Weiterbildung an Universitäten im ersten gesamtösterreichischen Universitätsentwicklungsplan 2016 bis $2021^{4}$ (BMWFW 2015a, S. 5) als dritte Säule der Lehre an Universitäten aufgeführt: Universitäre Lehre, Pädagogen-/Pädagoginnen-Ausbildung sowie Universitäre wissenschaftliche Weiterbildung. Letzterer wird dabei ein Beitrag zur „sozialen Durchlässigkeit“ zugewiesen und sie wird als „Antwort auf die demografische Entwicklung der Gesellschaft“ positioniert (ebd.).

\subsection{Zahlen und Fakten aus Österreich im Überblick}

Durch die Entwicklungen und Veränderungen begannen die österreichischen Universitäten zunehmend eine Zielgruppe in den Fokus zu nehmen, die als sogenannte „nicht-traditionelle“ Studierende bezeichnet werden. Damit sind vor allem Personen gemeint, die bereits Absolventinnen und Absolventen eines Hochschulstudiums sind, oder aber Personen, die zwar über keinen Studienabschluss verfügen, aber eine entsprechende mehrjährige Berufserfahrung nachweisen können.

Deutlich macht die Veränderung auch ein Blick auf die Zahlen an den Universitäten. Da nur eingeschränkte Daten für den wissenschaftlichen Weiterbildungsbereich in einer Zeitreihe abrufbar sind, werden jene der formalen wissenschaftlichen Weiterbildungsangebote (Universitätslehrgänge, die curricular verankert sind, d.h. durch die Universitätssenate eingerichtet worden sind) als Beispiel herangezogen. Seit dem WS 2004/05 ist dieser Bereich an den österreichischen Universitäten insgesamt um knapp 207\% angestiegen. Österreichweit besuchten im WS 2015/16 20.190 Studierende einen der Universitätslehrgänge in Österreich (Abb.1). Wobei hier deutlich die Donau-Universität Krems, die sich als Universität für Weiterbildung auf postgraduale Universitätslehrgänge spezialisiert hat, mit einem Studierendenanteil (WS 2015/16) von 44\% (oder 8.917 Personen) der größte Anbieter ist. 46\% der Weiterbildungsstudierenden in Österreich teilen sich auf 21 Universitäten auf (wobei 2014 zwei Universitäten keine Universitätslehrgänge anboten).

Als Vergleich dazu haben im WS 2014/15 österreichweit insgesamt 277.508 Personen ein „Regelstudium“ an einer der Universitäten (vgl. BMWFW 2015b, S.31) belegt. Dies bedeutet, dass der Anteil von Weiterbildungsstudierenden (in den Universitätslehrgängen) zu ordentlichen Studierenden der österreichischen Universitäten (ohne

4 Dieses Dokument bezieht sich auf Universitäten des Geltungsbereichs nach UG 2002 \6 und im Kontext des Weiterbildungsangebots auch auf die Donau-Universität Krems gem. DUK-Gesetz 2004, nicht im Fokus stehen die Privatuniversitäten (gem. PUG 2011). 


\section{Studierende in der wissenschaftlichen Weiterbildung}

an österreichischen Universitäten

25.000

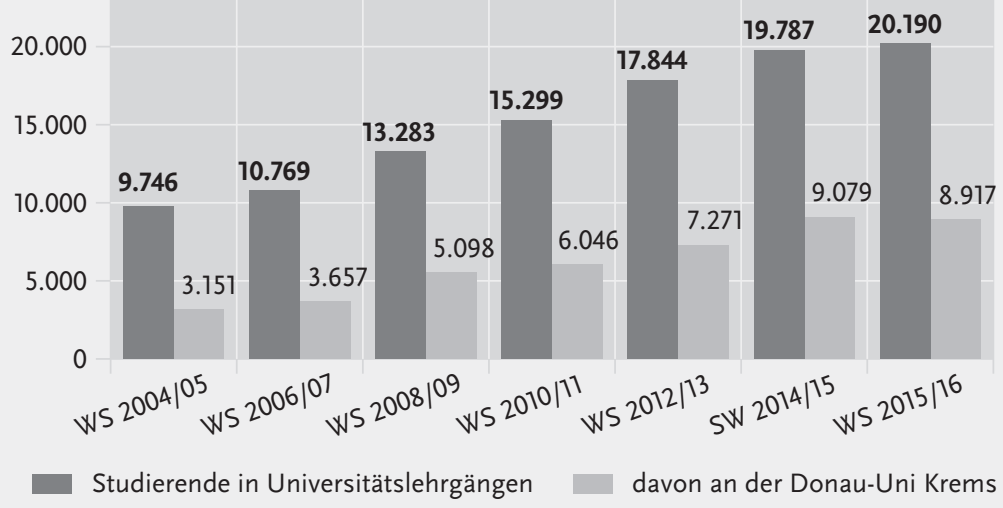

Abb. 1: Studierende in der wissenschaftlichen Weiterbildung an österreichischen Universitäten im Zeitverlauf

Quelle: BMWFW unidata; E. Gornik eigene Darstellung

Donau-Universität Krems, die ausschließlich Weiterbildungsstudierende hat) 3,7\% einnimmt.

Die Zunahme der Weiterbildungsstudierenden an den österreichischen Universitäten ist nur ein Beleg für die Entwicklung dieses Bereiches. Parallel dazu haben sich an den Universitäten eigene Einrichtungen entwickelt, die zunehmend auf- und ausgebaut wurden und sich mit den Formaten der wissenschaftlichen Weiterbildung beschäftigen. Diese Entwicklung führte dazu, dass sich AUCEN mit den Auswirkungen dieser Veränderungen und den institutionellen Ausdifferenzierungen beschäftigt hat. So waren in den letzten Jahren vor allem die organisatorische Verankerung, der personelle Aufbau und die Sichtbarmachung der wissenschaftlichen Weiterbildung innerhalb der eigenen Institution Fokusthemen bei den AUCEN-Meetings.

\subsection{Herausforderungen am Beispiel zweier hochschulübergreifender Projekte}

Qualitätsstandards zu entwickeln und zu sichern ist eine ständige Herausforderung in allen Organisationen. Im Rahmen des Netzwerkes AUCEN wird daher kontinuierlich an der Weiterentwicklung und Sicherung von Qualitätskriterien in den beiden Fachbereichen universitäre Weiterbildung und Personalentwicklung gearbeitet. AUCEN war und ist es ein Anliegen, sich in den diesbezüglichen Diskurs in Österreich aktiv einzubringen. Eine Möglichkeit, die sich in den letzten Jahren vermehrt geboten hat, war, Vertreterinnen und Vertreter von AUCEN in unterschiedlichsten Projekten als Expertinnen und Experten einzubinden. Diese Einbindung ist für das Netzwerk essenziell, da einerseits das Wissen der einzelnen AUCEN-Mitglieder in 
einen nationalen Diskurs eingebracht wird und andererseits die Erfahrungen aus den Projekten in das Netzwerk zurückfließen. Dies ermöglicht AUCEN die kontinuierliche Auseinandersetzung mit aktuellen bildungspolitischen Themen und Projekten sowie deren Weiterentwicklung. AUCEN-Mitglieder wirken unter anderem in Projekten der Agentur für Qualitätssicherung und Akkreditierung Austria (AQ Austria) mit, wie beispielsweise im Projekt „Qualitätsentwicklung der Weiterbildung an Hochschulen“, das 2011 vom Wissenschaftsministerium (BMWFW) unterstützt und von der AQ Austria begleitet wurde (vgl. AQA 2012).

\subsubsection{Qualitätsentwicklung der Weiterbildung an Hochschulen}

Der Ausgangspunkt dieses Projektes der AQ Austria fußte in der Segmentierung des österreichischen Hochschulsystems und den damit unterschiedlichen gesetzlichen Rahmenbedingungen und Regelungen zur externen Qualitätssicherung, die der wissenschaftlichen Weiterbildung zugrunde liegen. Die Vergabe akademischer Abschlüsse sollte jedoch an vergleichbare Standards gebunden sein, die hochschulübergreifend Gültigkeit beanspruchen, zusätzlich muss unter Berücksichtigung der Strategie des Lebenslangen Lernens sowie der Umsetzung des Bologna-Prozesses zukünftig insbesondere Aspekten wie der Durchlässigkeit der einzelnen Bildungssysteme sowie alternativen Zugängen zu Studienprogrammen verstärkte Beachtung geschenkt werden. Das durchgeführte Projekt konzentrierte sich auf das Segment der Universitätslehrgänge (die österreichische Bezeichnung für „Weiterbildende Studien“) mit Masterabschluss mit dem Ziel, Qualität und Wertigkeit von akademischen Abschlüssen in Hinblick auf die Entwicklung hochschulübergreifender Standards zu betrachten.

Wesentliche Ergebnisse und daraus resultierende Empfehlungen aus der Sicht von AUCEN waren (Auszug):

- Eine Orientierung an Learning Outcomes in der Entwicklung der weiterbildenden Studien und damit verbunden eine stärkere Gewichtung der Diagnose vorhandener Kompetenzen.

- Das Ermöglichen von alternativen Zugängen in weiterbildende Masterprogramme für Personen, die keinen ersten Hochschulabschluss vorweisen, jedoch über hochwertige berufliche Qualifikationen bzw. außerhochschulisch erworbene Kompetenzen verfügen.

- Der Aufbau von Beratungsleistungen für die Lernenden, um sie bei ihren individuellen Lernwegen zu unterstützen und eine Ausgestaltung der Studiengänge, die den zeitlichen Bedürfnissen der Lernenden entgegenkommt.

- Eine transparente Workload-Berechnung und entsprechend längere Dauer der Programme, welche berufsbegleitend angeboten werden.

Das österreichische Universitätsgesetz (UG 2002) unterscheidet zwischen regulären - oder auch ordentlichen - und außerordentlichen Studien; zu Letzteren gehören die Universitätslehrgänge, die der Weiterbildung dienen. Studierende von Universitätslehrgängen sind daher außerordentliche Studierende, auch wenn sie einen Weiterbildungslehrgang mit Masterabschluss belegt haben. Studierende weiterbildender 
Lehrgänge sind Studierenden in Regelstudien damit nicht gleichgestellt, z. B. bei der Vergabe von Stipendien. Durch die Verwendung des Begriffes „Universitätslehrgang“, der im internationalen Kontext unbekannt ist, entsteht allerdings eine Unklarheit, die internationalen Kooperationen wie z. B. den Joint Degree-Programme nicht zuträglich ist. Vor dem Hintergrund des Bologna-Prozesses empfahl die AQ Austria (2012, S.18), die Bezeichnung „Lehrgänge“ für weiterbildende Studien sowie den Status „außerordentlich“ für Studierende in Lehrgängen zu hinterfragen und die Studierenden hinsichtlich ihres Status jenen der Regelstudien gleichzustellen. Aus Sicht von AUCEN bildet dies einen wichtigen Ansatz in Hinblick auf die zukünftigen Entwicklungen und die immer wieder betonte zunehmende Bedeutung der Weiterbildung. Dies betrifft ebenfalls eine Überlegung in Richtung einer Erweiterung des Spektrums der Hochschul-Weiterbildung um sogenannte kostenpflichtige Undergraduate-Programme (also auf Bachelor-Niveau), die derzeit gesetzlich an Universitäten nicht möglich sind.

\subsubsection{Anerkennung und Anrechnung non-formal und informell erworbener Kompetenzen}

Die Notwendigkeit, sich aus Sicht von AUCEN mit dem Thema „Anerkennung nonformalen und informellen Lernens“ intensiv zu beschäftigen, ist durch die Forderungen in EU-Dokumenten (u.a. Europäisches Zentrum für die Förderung der Berufsbildung 2009) sowie national in der Strategie zum lebensbegleitenden Lernen (LLL:2020) oder Publikationen der österreichischen Universitätenkonferenz (vgl. Westphal/Friedrich 2009) gegeben. Für die wissenschaftliche Weiterbildung sind diese Fragen nicht neu und können als „gelebte“ Expertise - mit Empirie hinterlegt (vgl. Kil 2015) - eingebracht werden. In welchem Ausmaß und welche Instrumente an den österreichischen Hochschulen für die Anerkennung und Anrechnung Verwendung finden, werden in einem seit Juli 2014 andauernden Projekt der AQ Austria unter aktiver Mitarbeit von AUCEN-Mitgliedsuniversitäten, finanziert durch das österreichische Wissenschaftsministerium, untersucht. Die Projektergebnisse insgesamt sind in einer Broschüre der AQ Austria in 2016 veröffentlicht und als PDF-File erhältlich. 2015 wurden im Rahmen der Jahrestagung der AQ Austria die Zwischenergebnisse präsentiert, die in der Tagungsdokumentation publiziert wurden (vgl. Gornik/Freiberger/Birke 2016, S.125 ff.).

Aus Sicht von AUCEN ist die Beschäftigung mit dieser Thematik für die Zukunft und Entwicklung, nicht nur der wissenschaftlichen Weiterbildung, sondern der gesamten hochschulischen Bildung, essenziell. Gerade in der Weiterbildung zeigen sich oftmals Tendenzen frühzeitig auf, die Jahre oder sogar Jahrzehnte später in den „Regelstudien-Bereich“ Einzug finden (beispielsweise Blended-Learning-Konzepte, neue didaktische Formate, Beratungs- und Serviceleistungen für berufsbegleitende Studierende, etc.). Aus diesem Grund bilden die Erfahrungen, die an den österreichischen hochschulischen Weiterbildungseinrichtungen gemacht wurden, einen wesentlichen Baustein - gerade zum Thema der Anerkennung von non-formal und informell erworbenen Kompetenzen. Es zeigt sich, dass die Diskussionen an den 
österreichischen Hochschulen zu diesem Thema, geprägt durch zwei Faktoren, einseitig verlaufen: vonseiten der Hochschulleitungen werden Argumente eingebracht, die die mangelnde Finanzierung von Verfahren in den Mittelpunkt rücken sowie die Tatsache, dass - aufgrund des freien Hochschulzugangs - an vielen Universitäten kein Mangel an Studierenden herrscht. Umso mehr setzt sich AUCEN mit seinen Vertreterinnen und Vertretern dafür ein, Bewusstseinsbildung für Themen anzustoßen, die (noch) nicht im Fokus aller Hochschulleitungen liegen.

Die beschriebenen Projekte zeigen auf, dass die österreichischen Universitäten oftmals ganz unterschiedlich mit Herausforderungen umgehen (wollen). Inwiefern die Diskussion und gemeinsame Arbeit im österreichischen Netzwerk AUCEN trotz unterschiedlicher Herausforderungen, Interessen und Strategien gewinnbringend sein kann, wird im folgenden Abschnitt thematisiert.

\section{Eigeninteressen der Universitäten versus kooperative Zusammenarbeit - ein produktives Spannungsfeld im AUCEN-Netzwerk}

Netzwerke, seien sie universitätsintern oder universitätsübergreifend, bieten Möglichkeiten des Austausches von Wissen, Expertise und Know-how. Neben dem fachlichen Input spielen der wertvolle Gedanken- und Erfahrungsaustausch aufgrund gemeinsamer Strukturen und Zielgruppen, die Diskussion über universitätsspezifische Inhalte und Formate, die Eröffnung von Perspektiven durch kreatives Miteinander-Denken, der Wissenstransfer und die Ideengenerierung eine Rolle bei der Netzwerkarbeit.

Auf der anderen Seite stehen etliche Herausforderungen im Raum. So befinden sich Universitäten in einem steten Konkurrenzkampf um die „besten“ Studierenden, die „exzellentesten“ Wissenschaftlerinnen und Wissenschaftler, die „innovativsten“ Forscherinnen und Forscher, um die Erhöhung der Budgetmittel und vieles mehr. Darüber hinaus arbeitet jede Universität an ihrem Profil, um bei internationalen Rankings top platziert zu sein, aber auch - um in der wissenschaftlichen Weiterbildung zu bleiben - zahlende Studierende zu lukrieren. Dieser Auftrag der Universitäten führt unausweichlich zu Wettbewerb und Konkurrenz untereinander.

Eigeninteressen der Universitäten versus gemeinsamer inhaltlicher Diskurs und Kooperation - der Gegensatz könnte nicht größer sein. Und dennoch: Im österreichischen Netzwerk für universitäre Weiterbildung und Personalentwicklung (AUCEN) stehen der Austausch von Informationen, das Zurverfügungstellen von Expertise sowie das gemeinsame Erarbeiten von Konzepten und Ideen im Vordergrund. In den beiden Arbeitsgruppen „Wissenschaftliche Weiterbildung“ und „Personalentwicklung“ wird in Form von kollegialer Beratung systematisch und strukturiert Wissen ausgetauscht, Know-how transferiert und dadurch Anregungen für die Arbeit an den jeweiligen Universitäten gegeben. Dass die von den Mitgliedern vorgestellten Kon- 
zepte an einer anderen Universität aufgrund der - bei allen Gemeinsamkeiten, aber dennoch - heterogenen Strukturen an den Universitäten nicht 1:1 umsetzbar sind, ist als Prämisse anzusehen. Dennoch werden neue Ideen und vorgestellte Beispiele "guter Praxis" aufgenommen, analysiert, adaptiert und finden sich in der ein oder anderen Form an den Universitäten wieder. Die Zusammenarbeit in den beiden Fachbereichen "Wissenschaftliche Weiterbildung" und "Personalentwicklung“ führt zu belebendem Austausch sowie fallweise zu gemeinsamen Initiativen zwischen den Universitäten.

Im Spannungsfeld „Eigeninteresse vs. Kooperation“ geht die Zusammenarbeit zwischen den Universitäten im AUCEN-Netzwerk ganz klar als Gewinnerin hervor. Warum? Eine Antwort liegt darin begründet, dass sich die Netzwerkmitglieder über die Wichtigkeit des „Über-den-Tellerrand-Schauens“ einig sind, ebenso wie das Anliegen einer qualitätsvollen und professionellen Weiterentwicklung in universitärer Personalentwicklung und Weiterbildung im Vordergrund steht - verbunden mit dem Wissen, dass die Organisationsstruktur von Universitäten ein Spezifikum ist, welches ihrerseits wiederum ein verbindendes Element darstellt. Eine gemeinsame Strategie bzw. abgestimmte Positionen durch den AUCEN-Vorstand in die Öffentlichkeit zu transportieren, verleiht den Anliegen der universitären Personalentwicklung und Weiterbildung Öffentlichkeitswirksamkeit und führt zu einer verstärkten Präsenz der Universitäten in diesem Bereich.

\section{Ausblick}

Die österreichischen Universitäten haben seit Inkrafttreten des Universitätsgesetzes, der „Entlassung“ der Universitäten in die Vollrechtsfähigkeit und der damit verbundenen Autonomie im Jahr 2004 eine starke Transformation durchlaufen und sahen - und sehen sich noch immer - mit Herausforderungen in bildungspolitischer sowie auch in struktureller Hinsicht in den Institutionen selbst konfrontiert. Die Vertreterinnen und Vertreter von AUCEN sind nicht nur an diesem Transformationsprozess beteiligt, sondern gestalten diesen aktiv mit. Die Stärkung der Qualität und der Durchlässigkeit in der wissenschaftlichen Weiterbildung beispielsweise ist im bis 2021 umzusetzenden österreichischen Universitätsentwicklungsplan (BMWFW 2015a, S.13 ff.) als eines der wesentlichen Ziele zur Verbesserung der Qualität in der universitären Lehre festgelegt worden. Daher wird sich auch AUCEN in den kommenden zwei Jahren diesen Bereichen verstärkt widmen. Für die Umsetzung desselben ist AUCEN sowohl im Bereich der Personalentwicklung (vgl. vor allem Systemziel 5 „Förderung des wissenschaftlichen Nachwuchses mit dem Fokus auf das universitäre Personalmanagement", S. 7) als auch im Bereich der wissenschaftlichen Weiterbildung im Systemziel 3 "Verbesserung der Qualität der universitären Lehre“ (S.5) gut vorbereitet. Im Systemziel 8 „Förderung eines Kulturwandels zugunsten von sozialer Inklusion, Geschlechtergerechtigkeit und Diversität an der Universität" (S. 8) ist die Zusammenarbeit der beiden Fachbereiche „Personalentwicklung“ und 
„Weiterbildung“ - im Sinne des originären Anliegens von AUCEN - künftig gefordert.

\section{Literatur}

AQA - Österreichische Qualitätssicherungsagentur (2016): Anerkennung und Anrechnung non-formaler und informell erworbener Kompetenzen - Empfehlungen zur Gestaltung von Anerkennungs- und Anrechnungsverfahren. Wien: Facultas Verlags- und Buchhandels AG.

AQA - Österreichische Qualitätssicherungsagentur (2012): Qualitätsentwicklung der Weiterbildung an Hochschulen. Wien: Facultas Verlags- und Buchhandels AG.

BMWFW (2014): Universitätsbericht 2014. http://wissenschaft.bmwfw.gv.at/bmwfw/ ministerium/veranstaltungenpublikationen/publikationen/wissenschaft/universitaets wesen/hochschul-und-universitaetsberichte/ [Zugriff: 16.04.2016]

BMWFW (2015a): Der gesamtösterreichische Universitätsentwicklungsplan 2016 - 2021. http://wissenschaft.bmwfw.gv.at/fileadmin/user_upload/wissenschaft/publikationen/ 2015-12-03_goe_UEP-Kurz.pdf [Zugriff: 16.04.2016]

BMWFW (2015b): 2015 > Statistisches Taschenbuch. http://wissenschaft.bmwfw.gv.at/file admin/user_upload/wissenschaft/publikationen/Stat_TB_2015.pdf [Zugriff: 16.04.2016]

BMWFW unidata - Zahlen und Fakten auf Knopfdruck. https://oravm13.noc-science.at/ apex/f?p=103:36:0::NO: [Zugriff: 16.04.2016]

Europäisches Zentrum für die Förderung der Berufsbildung (2009): Europäische Leitlinien für die Validierung nicht formalen und informellen Lernens. Luxemburg: Amt für Veröffentlichungen der Europäischen Union. Abzurufen unter www.cedefop.europa.eu/ files/4054_de.pdf [Zugriff: 04.05.2016]

Gornik, E./Freiberger, D./Birke, B. (2016): Sichtbarmachung von non-formal und informell erworbenen Kompetenzen: Eine aktuelle Einschätzung mit Fokus auf hochschulische Weiterbildungsprogramme. In: AQ Austria (Hrsg.): Gutes Lernen und gute Lehre. Welchen Beitrag leistet die Qualitätssicherung? Beiträge zur 3. AQ Austria Jahrestagung 2015. Wien: Facultas, S. 125-137.

Gornik, E./Steiger, A. (2015): Personalentwicklung an österreichischen Universitäten Was kann das österreichische Netzwerk AUCEN (Austrian University Continuing Education and Staff Development Network) leisten? In: Personal- und Organisationsentwicklung in Einrichtungen von Lehre und Forschung. 2015(2+3), S. 39-43.

Kil, M. (2016): Wissenschaftliche Weiterbildung an Universitäten: Zur Komplementarität und zum gesellschaftlichen Nutzen im System Lebenslangen Lernens am Beispiel der Donau-Universität Krems. In: Weiterbildung - Zeitschrift für Grundlagen, Praxis und Trends, Schwerpunktheft „Universitäre Weiterbildung“, 2016(1), S.12-15. 
LLL:2020 - Strategie zum Lebensbegleitenden Lernen in Österreich. https://www.bmbf. gv.at/ministerium/vp/2011/1llarbeitspapier_ebook_gross_20916.pdf?4dtiae][Zugriff: 12.04.2016]

Privatuniversitätengesetz (2011): https://www.jusline.at/Privatuniversitaetengesetz_(PUG). html [Zugriff: 18.05 .2016$]$

Universitätsgesetz (2002): https://www.jusline.at/Universitaetsgesetz_(UnivG).html [Zugriff: 03.05.2016]

Westphal, E./Friedrich, M. (Hrsg.) (2009): Anerkennung von non-formalem und informellem Lernen an Universitäten. Graz: Leykam. 



\section{Wirkungsvolle Interessenvertretung der universitären Weiterbildung in der Schweiz: Swissuni}

ANDREAS FISCHER

„Swissuni - Universitäre Weiterbildung Schweiz“ ist die Organisation, die in der Schweiz die universitäre Weiterbildung repräsentativ vertritt. Mitglieder sind die Weiterbildungsstellen aller universitären Hochschulen der Deutschschweiz, der Romandie, des Tessins und des Fürstentums Liechtenstein. Swissuni wurde 2002 als Nachfolgeorganisation der seit 1991 aktiven Arbeitsgruppe Weiterbildungsstellen der Schweizerischen Hochschulkonferenz gegründet.

\section{Hochschulweiterbildung in der Schweiz}

Die Hochschulweiterbildung der Schweiz wird getragen von zwölf universitären Hochschulen, neun Fachhochschulen, 17 Pädagogischen Hochschulen und drei Hochschulinstituten (vgl. Fischer 2014). Sie wird in den Formaten Master of Advanced Studies (MAS, mindestens 6o ECTS-Punkte), Diploma of Advanced Studies (DAS, mindestens 30 ECTS-Punkte), Certificate of Advanced Studies (mindestens zehn ECTS-Punkte) sowie als Weiterbildungskurse angeboten. Darüber hinaus offerieren die Hochschulen mehr und mehr Distance Education-Angebote, MOOCs oder speziell für Unternehmen auch Customized Programmes. Eine vollständige statistische Übersicht über das Angebot und die Teilnehmerzahlen existiert nicht. Für 2014/2015 führt das Bundesamt für Statistik auf Stufe MAS an Universitäten 5.534 immatrikulierte Studierende und 1.815 Abschlüsse auf (7.502 Immatrikulierte und 2.693 Abschlüsse an Fachhochschulen und Pädagogischen Hochschulen).' Im Dezember 2015 waren bei den Mitgliedsuniversitäten von Swissuni 181 MAS-, 133 DAS- und 362 CAS-Studiengänge online ausgeschrieben. ${ }^{2}$ Rechtlich stützt sich

\footnotetext{
1 Bundesamt für Statistik: http://www.bfs.admin.ch/bfs/portal/de/index/themen/15/06/tab/blank/uebersicht.html [Zugriff: 07.03.2016]

2 Eigene Recherche der Webseiten am 10.12.2015
} 
die Hochschulweiterbildung auf das Hochschulförderungs- und -koordinationsgesetz (HFKG) und das Weiterbildungsgesetz (WeBiG) des Bundes sowie auf die kantonalen Hochschulgesetze ab, die die Weiterbildung als eine der Aufgaben der Hochschulen definieren. Die Hochschulweiterbildung hat im Grundsatz kostendeckend angeboten zu werden, was sich in ihrer Marktorientierung und letztlich der Preisgestaltung ausdrückt. Das heißt, dass die Kurse und Programme vollumfänglich über die Teilnahmegebühren finanziert werden müssen, weshalb sie markant teurer sind als die Studienangebote der Grundausbildung.

\section{Aktuelles Porträt des Vereins Swissuni}

„Swissuni - Universitäre Weiterbildung Schweiz“ ist ein gemeinnütziger Verein mit dem Zweck der Zusammenarbeit der Weiterbildungsstellen der Universitäten. Er fördert gemäß Statuten den Informations- und Erfahrungsaustausch, entwickelt die Zusammenarbeit untereinander und mit externen Organisationen, arbeitet mit Swissuniversities ${ }^{3}$ zusammen, realisiert und koordiniert Maßnahmen im Bereich der Öffentlichkeitsarbeit, koordiniert die Entwicklung von Weiterbildungsangeboten und fördert die diesbezügliche Zusammenarbeit, macht Vorschläge zur Bildungspolitik im Weiterbildungsbereich und führt gemeinsame Projekte durch. Mitglieder sind die 13 Leiterinnen und Leiter der Weiterbildungsstellen der schweizerischen und liechtensteinischen universitären Hochschulen. Der Verein arbeitet ehrenamtlich und wird von einem zweiköpfigen Vorstand geleitet. Er führt eine Internetplattform zur internen Kommunikation und eine Website mit integrierter Kursdatenbank (www.swissuni.ch). In der Regel finden jährlich drei bis vier Sitzungen statt, davon zwei bis drei in der zentral gelegenen Bundeshauptstadt Bern und die zweitägige Jahresversammlung abwechselnd bei einer der Mitgliedsuniversitäten. Spezielle Themen werden in einzelnen Arbeitsgruppen bearbeitet. Aktuell stehen folgende Themen auf der Agenda: Alleinstellungsmerkmale der universitären Weiterbildung, Zusammenarbeit mit Swissuniversities sowie den Fachhochschulen und Pädagogischen Hochschulen, Flüchtlingssituation, Urheberrechtsabgaben, ISCED-Klassifizierung der Weiterbildungsabschlüsse, Neugestaltung der Website und der Datenbank.

\section{Institutionelle Bedeutung von Swissuni}

Swissuni deckt die universitäre Weiterbildung repräsentativ ab und ist die offizielle Ansprechstelle für Swissuniversities in Sachen universitäre Weiterbildung. Da Swissuniversities als Nachfolgeorganisation der drei Rektorenkonferenzen der Universitäten/ETHs, der Fachhochschulen sowie der Pädagogischen Hochschulen erst kürzlich gegründet wurde, ist die vertikale Integration der Hochschulweiterbildung

3 Swissuniversities ist die Rektorenkonferenz der schweizerischen Hochschulen (vgl. http://www.swissuniversities.ch/, Zugriff: 14.03.2016) 
in diesem Organ noch nicht vollständig zur Zufriedenheit der Weiterbildungsstellen geregelt. Auf horizontaler Ebene bestehen Kooperationsabsichten zwischen den Delegationen der Weiterbildung dieser drei Hochschultypen, die aber noch nicht in eine funktionierende Organisation umgesetzt sind. Über ihre Mitglieder ist Swissuni national und international gut vernetzt, so beispielsweise mit der schweizerischen Conférence Romande de la Formation Continue, der Schweizerischen Gesellschaft für Bildungsforschung, dem Swiss Faculty Development Network (Hochschuldidaktik), dem European University Continuing Education Network, der Deutschen Gesellschaft für wissenschaftliche Weiterbildung und Fernstudium, der Europäischen Gesellschaft für Ingenieur-Ausbildung SEFI, der International Association for Continuing Engineering Education IACEE sowie mit verschiedenen weiteren Fachgesellschaften.

\section{Entstehung von Swissuni}

Der Bund unterstützte mit seinen Sondermaßnahmen zugunsten der universitären Weiterbildung von 1990 bis 1999 die universitären Hochschulen mit dem Zweck des Aufbaus von Weiterbildungsstellen und eines Studienangebots (vgl. Weber/Fischer 1992). Im Rahmen dieser Maßnahmen haben sich die Verantwortlichen der Weiterbildungsstellen ab 1991 zu regelmäßigen Arbeitssitzungen getroffen. „Diese Leiterinnen- und Leitertreffen dienen dem Austausch von Informationen und Erfahrungen. Absprachen ermöglichen teilweise einheitliche Vorgehensweisen, die den administrativen Ablauf von Studiengängen und vor allem auch die Zusammenarbeit der Koordinationsstellen respektive der Hochschulen vereinfachen. Im Rahmen dieser Treffen wird die Realisierung gemeinsamer Projekte (z. B. gesamtschweizerische Kursübersicht auf Videotext, Aufbau einer Modulbörse) vorbereitet. Weitere Ziele sind die fachliche Auseinandersetzung mit verschiedenen Themenschwerpunkten und ein gemeinsames Auftreten gegenüber den Behörden" (Schweizerische Hochschulkonferenz 1993, S. 7). Aufgrund einer Evaluation der Arbeit der Weiterbildungsstellen (Knaus et al. 1994) ist diese Zusammenarbeit 1994 in Form einer Arbeitsgruppe der Weiterbildungskommission der Schweizerischen Hochschulkonferenz institutionalisiert worden. 2001 wurden die nationalen Universitätsgremien neu organisiert; dabei verlor jedoch die Arbeitsgruppe ihre institutionelle Anbindung. Die Mitglieder - überzeugt von der Notwendigkeit einer organisierten Zusammenarbeit - gründeten 2002 den Verein Swissuni, der seither den Kontakt zur Rektorenkonferenz der Schweizerischen Universitäten und deren Nachfolgeorganisation Swissuniversities über eine ständige Gastmitgliedschaft derselben aufrechterhält. 


\section{Ergebnisse der Tätigkeit von Swissuni}

Swissuni hat sich - meist auf eigene Initiative hin - mit einer Vielzahl von Themen beschäftigt und konnte in vielen Fällen wichtige Resultate erreichen, die sich förderlich für die Entwicklung der universitären Weiterbildung auswirkten. Nachfolgend werden sieben Aufgabenbereiche beschrieben, bei denen Swissuni erfolgreich war, gefolgt von zwei schwierigen Feldern, auf denen die erhofften Ergebnisse noch auf sich warten lassen.

\subsection{Kommunikation}

Im Bereich Kommunikation war eine der ersten Aufgaben die Einrichtung einer gemeinsamen Internetplattform zur Publikation der Angebote (nach mehreren Vorgängerauftritten aktuell: www.swissuni.ch), zudem wurden einige gemeinsame Messeauftritte organisiert. Die Website ist noch heute das zentrale Instrument des Außenauftritts von Swissuni und gleichzeitig eine Dienstleistung für die Mitgliedsuniversitäten, die dort ihr Angebot bekanntmachen können.

\subsection{Qualität}

Ein zentrales Thema, das über die ganze Zeit immer wieder diskutiert wurde, ist die Qualität der universitären Weiterbildung. Als Ergebnis dieser Arbeiten wurden 1996 Qualitätsgrundsätze ${ }^{4}$ formuliert, auf die sich alle Universitäten verpflichteten. 2009 publizierte Swissuni die „Empfehlungen für die Qualitätsentwicklung in der universitären Weiterbildung ${ }^{\prime 5}$ und 2013 genehmigte die Schweizerische Universitätskonferenz spezielle Standards für die Akkreditierung von Weiterbildungsstudiengängen, die auf Initiative von Swissuni formuliert wurden.

\subsection{ECTS}

Mit der Einführung des ECTS-Systems in der universitären Ausbildung wurde dessen Umsetzung in der Weiterbildung zu einem dringenden Thema. Swissuni erarbeitete dazu 1998 Empfehlungen $^{6}$, die auch heute noch eine wichtige Grundlage sind.

\subsection{Harmonisierung der Formate}

Die rasante Entwicklung der Weiterbildungsangebote aufgrund der Bundesförderung verlief ab 1990 bezüglich Formaten und Abschlussbezeichnungen zunächst sehr ungeordnet und führte zu einer unübersichtlichen Situation. In engem Kontakt mit der Hochschulkonferenz diskutierte die Arbeitsgruppe ab 1998 über eine Har-

4 www.swissuni.ch/documents/Qualitatsgrundsatze_1996.pdf [Zugriff: 14.03.2016]

5 www.swissuni.ch/documents/Qualitatskriterien_2010.pdf [Zugriff: 14.03.2016]

6 Version 2005: www.swissuni.ch/documents/ects_DE.pdf [Zugriff: 14.03.2016] 
monisierung und führte 1990 eine Bestandsaufnahme durch. Parallel dazu veränderte sich mit den Bologna-Reformen die europäische Hochschullandschaft nachhaltig, wobei jedoch die Weiterbildung weitestgehend vergessen ging. Swissuni formulierte daher bald Empfehlungen zu neuen, „Bologna-tauglichen“ Weiterbildungsformaten, die schliesslich 2004 von den zuständigen Instanzen auf Bundesebene als Empfehlungen in Kraft gesetzt wurden und vier Formate definierten: ein- oder mehrtägige Weiterbildungskurse, Zertifikatslehrgänge (mind. 150 Kontaktstunden), Diplomlehrgänge (mindestens 300 Kontaktstunden), Master of Advanced Studies (MAS)-Programme (mindestens 6o ECTS-Punkte). In der Neuauflage der Empfehlungen $2008^{7}$ wurde der Umfang der Zertifikatslehrgänge auf mindestens zehn ECTS-Punkte und derjenige der Diplomlehrgänge auf mindestens 30 ECTSPunkte festgelegt. Im Nationalen Qualifikationsrahmen für den Hochschulbereich (2009) schließlich wurden die von Swissuni vorgeschlagenen Abschlussbezeichnungen Diploma of Advanced Studies (DAS) und Certificate of Advanced Studies (CAS) zusätzlich zu den MAS verbindlich festgelegt, nachdem schon ein paar Jahre früher erste Studiengänge mit diesen Bezeichnungen eingeführt wurden. Da wenig später nicht zuletzt auch aufgrund von Vorarbeiten von Swissuni die Fachhochschulen und Pädagogischen Hochschulen diese Typisierung ebenfalls einführten, hat sich diese klare Strukturierung der Formate schnell im schweizerischen Hochschulraum und auf den Arbeitsmärkten durchgesetzt und findet auch im deutschsprachigen Ausland Beachtung. So ist diese denn auch der Anlass für die grenzüberschreitende $\mathrm{Zu}$ sammenarbeit mit der Albrecht-Ludwig-Universität Freiburg, die zu einer Gastmitgliedschaft der dortigen Weiterbildungsstelle geführt hat.

\subsection{Immatrikulation}

2004 erreichte Swissuni mit einer Arbeitsgruppe der Rektorenkonferenz, dass alle MAS-Studierenden immatrikuliert und damit in der Hochschulstatistik des Bundesamts für Statistik erfasst werden.

\subsection{Nationaler Qualifikationsrahmen}

Swissuni hat sich 2007 bis 2009 in die Erarbeitung des Nationalen Qualifikationsrahmens für den Hochschulbereich (nqf.ch-HS) ${ }^{8}$ eingebracht und konnte bewirken, dass die Weiterbildungsstudiengänge mit entsprechenden Deskriptoren integriert sind.

7 www.swissuniversities.ch/fileadmin/swissuniversities/Dokumente/DE/UH/Empfehlungen/Empfehlungen_ CRUS_2010_2_d.f-6.pdf, S. 40-42 [Zugriff: 14.03.2016]

8 www.swissuniversities.ch/de/hochschulraum/qualifikationsrahmen/weiterbildung/ [Zugriff: 14.03.2016] 


\subsection{Weiterbildungsgesetz}

Bei der Erarbeitung des Bundesgesetzes über die Weiterbildung ${ }^{9}$ (2009-2014) konnte SwissUni durch Mitarbeit und im Hinblick auf die parlamentarische Behandlung mit intensivem Lobbyieren bewirken, dass ihre Interessen gewahrt blieben und insbesondere der Wettbewerbsartikel nicht zuungunsten der Hochschulweiterbildung formuliert wurde (vgl. auch Fischer 2013, S. 84).

\subsection{Weiterbildungsstatistik}

Die Arbeitsgruppe und später Swissuni unternahmen mehrere Anläufe zur Verbesserung der statistischen Lage der universitären Weiterbildung. Diese scheiterten bisher alle. Es gelang aufgrund der unterschiedlichen Datenlagen an den einzelnen Universitäten nicht einmal, eine minimale gemeinsame Statistik unter den Mitgliedern aufzubauen. Dies ist umso bedauerlicher, als die Integration der universitären Weiterbildung in die Weiterbildungs- wie auch die Hochschulstatistiken des Bundes nur lückenhaft ist.

\subsection{Hochschulpolitische Repräsentanz}

2006 wurde in einer Evaluation der universitären Weiterbildung empfohlen: „Innerhalb des Sektors universitäre Weiterbildung sollte, über die freiwillige Abstimmung unter den Leitern der Weiterbildungsstellen hinaus, eine nationale Arbeitsgruppe die strategische und operative Sicht der universitären Weiterbildung zusammenbringen (d.h. z. B. verantwortliche Vizerektoren und die Leiter der Weiterbildungsstellen), um best practice und positive Erfahrungen mit der Nutzung des innovativen Potentials der Weiterbildung auszutauschen und eine möglichst proaktive Haltung zur universitären Weiterbildung der Schweiz, welche Weitsicht und Verständnis geeigneter Instrumente zu deren Verwirklichung vereint, zu fördern" (Reichert 2006, S. 52). Das Anliegen von Swissuni, die Interessen der universitären Weiterbildung auf der Ebene der Rektoren vertreten zu wissen, besteht seit Jahren. Mit der Weiterbildungskommission der Schweizerischen Hochschulkonferenz gab es zwischen 1990 und 1995 eine starke Vertretung auch auf hochschulpolitischer Ebene, die dann aber nicht weitergeführt wurde. Zwischen 2011 und 2014 war in der Rektorenkonferenz einer der Rektoren Delegierter für Weiterbildung. Seither ist die Repräsentanz der Weiterbildung auf höherer Stufe wieder pendent, die Hochschulweiterbildung ist in die Delegation Lehre integriert und im Organigramm von Swissuniversities nur in Klammern sichtbar („Aus- und Weiterbildung“).

9 https://www.admin.ch/opc/de/federal-gazette/2014/5177.pdf [Zugriff: 14.03.2016] 


\section{Zukünftige Herausforderungen}

Swissuni hat sich in den letzten gut 25 Jahren als sehr wohl in der Lage gezeigt, trotz der direkten Konkurrenz der Mitglieder als Anbieter auf dem Weiterbildungsmarkt die Gegensätze der unterschiedlichen Größen, Interessen und Ausrichtungen der Mitgliedsuniversitäten und der unterschiedlichen sprachlichen und kulturellen Eigenheiten der einzelnen Landesteile zum Nutzen aller Beteiligten immer wieder überwinden zu können. Dabei ist es gelungen, bei aller Konzentration auf die nationale Situation international offen zu sein und über die Mitglieder Kontakte zu anderen Verbänden im Ausland und auf europäischer und globaler Ebene aufrechtzuerhalten. Damit besteht eine solide Basis, um den zukünftigen Herausforderungen zuversichtlich begegnen zu können. Aus heutiger Sicht stehen prioritär folgende Aufgaben bevor:

- die Verstärkung der gemeinsamen Kommunikation nach außen, vor allem über die Website und deren Promotion;

- die Klärung der Position zu den wichtigsten Konkurrenten, den Fachhochschulen;

- die verstärkte Einflussnahme in den hochschulpolitischen Gremien;

- die maßgebliche Mitgestaltung der durch das Hochschulförderungs- und -koordinationsgesetz geforderten einheitlichen Richtlinien für die Hochschulweiterbildung mit Aussagen zur Qualitätssicherung, zum Wettbewerb, zur gegenseitigen Anerkennung und zu den Formaten;

- der Einsatz für die Integration der universitären Weiterbildung in den Nationalen Qualifikationsrahmen;

- die Mitarbeit bei der Umsetzung eines umfassend verstandenen Konzepts des Lebenslangen Lernens in den Universitäten, da voraussehbar ist, dass damit die Grenzen zwischen Aus- und Weiterbildung verwischt werden.

\section{Literatur}

Fischer, A. (2014): Hochschulweiterbildung in einem heterogenen Feld. Arbeitsdokument Geschäftsstelle SWIR 3/2014. Bern: Schweizerischer Wissenschafts- und Innovationsrat.

Fischer, A. (2013): Swissuni 2013: von schädlichen Gesetzesartikeln, nützlichen Qualitätsstandards und engagierten Auseinandersetzungen. In: Hochschule und Weiterbildung 2013(2), S. 84 .

Knaus, A. et al. (1994): Die Arbeit der Weiterbildungsstellen an den kantonalen Hochschulen der Schweiz: Schlussbericht. Bern (nicht publiziert). 
Reichert, S. (2006): Universitäre Weiterbildung in der Schweiz: Bestandsaufnahme und Perspektiven im europäischen Vergleich. Studie im Auftrag des Staatssekretariats für Bildung und Forschung. Bern (nicht publiziert).

Schweizerische Hochschulkonferenz, Weiterbildungskommission (1993): Sondermassnahmen universitäre Weiterbildung, Zwischenbericht. Dokument 35193 . Bern (nicht publiziert).

Weber, K./Fischer, A. (1992): Die universitäre Weiterbildungsinitiative der schweizerischen Regierung. Ein erfolgreiches Instrument in einem föderalistisch organisierten Hochschulwesen. In: Beiträge zur Hochschulforschung, Bayerisches Staatsinstitut für Hochschulforschung und Hochschulplanung, München, S. 441-465. 


\section{Working together to promote University Lifelong learning in Europe: eucen}

FRANÇOISE DE VIRON

\section{Introduction}

Nowadays, collective knowledge, continuous learning and collaborating and learning in network are the most appropriate answer to complexity. Our environments at European, national or regional levels are rapidly changing and continuously evolving. Changes are coming from diverse perspectives: political, social, cultural, economic, and technological.

It implies that universities have to be able to continuously detect, analyse and understand these changes and they have to adapt in order to offer provisions and services to their audience -people, communities, organisations- in order to increase their learning capacity and develop their abilities to face the changes. Universities are new spaces for learning opportunities lifelong and life-wide where a new culture of learning is required.

This chapter presents eucen, a specific European university lifelong learning network. Using an organisational framework, based on the concepts of collaborative inter-organisational network and community of practice, it describes the characteristics, the functions and benefits of this network and highlights some prerequisites for its development. It concludes by a short test application of the framework to other networks dedicated to LLL at national level and highlights some examples in the case of Germany and the DGWF network.

\section{What is lifelong learning at university level?}

Based on its 25 years of practices and on results of European projects surveys (Davies 2007; Davies 2010), eucen proposes the following definition of lifelong learning at university level. 
University Lifelong Learning (ULLL) is the provision by higher education institutions of learning opportunities, services and research for:

- the personal and professional development of a wide range of individuals - lifelong and life-wide

- and the social, cultural and economic development of communities and the region

- It is at university level and research-based; it focuses primarily on the needs of the learners; and it is often developed and/or provided in collaboration with stakeholders and external actors.

Another European project led by eucen, DIALOGUE (de Viron 2014), identifies emergence factors for ULLL development in different European countries: the demographic changes, the increasing number of students, the pressure from labour market demands, and health care considered as strong drivers to develop ULLL. These factors are augmented by the appearance of new private universities in some countries. The emergence of the knowledge society is also outlined by DIALOGUE project partners, as it implies a major change in knowledge production and recognising that other actors besides the universities are engaged with it. "This change forces the university to dialogue in general. In this context, academic research has a crucial role to play in validating methods used to co-create knowledge and to articulate and structure fragmented knowledge into a language that is understood by all" (de Viron 2014, p. 5). Therefore, University Lifelong Learning (ULLL) represents "a radical conceptual shift in thinking about education; it is distinguished for its capacity to subsume all forms of learning-formal, informal and non-formal and to provide opportunity to progress from informal learning to accredited learning in universities" (de Viron 2014, p. 5).

Successive EUA reports (Sursock/Smidt 2010; Surscock 2015) highlight and comment the diversity in the development of University Lifelong Learning throughout European countries. What is called 'lifelong learning' in some countries might be called 'adult education', 'postgraduate studies' or 'continuing education' in others, and the content under the label vary from one country to another (de Viron/Davies 2015). The great variety of lifelong learners is also emphasised, such as young adults without a university degree, individuals seeking professional development, unemployed adults, migrants, and so on.

However, Sursock (2015) notices a strong progression in the number of European universities active in lifelong learning since 2010. "Lifelong learning strategies are in place in $65 \%$ of institutions and in the planning stage for a further $24 \%$; $9 \%$ indicate that they are not planning one" (Sursock 2015, p. 67). This result is based on a questionnaire to which 451 higher education institutions in 48 countries responded. 


\section{3 eucen, a network}

In the University Lifelong Learning context, which is new, complex and diverse, a collaborative approach is needed for professionals. It is the main reason why eucen, European University Continuing Education Network, is created in 1991. eucen is a non-for-profit association, currently comprising 178 members, active in lifelong learning or willing to be active, from 35 European countries. Amongst these members, 150 are universities and 17 are national university networks as DGWF, the German network.

The main goals of eucen are twofold: first to contribute to the social, economic and cultural life of Europe through the promotion and advancement of lifelong learning within higher education institutions in Europe and elsewhere; second to foster universities' influence in the development of lifelong learning knowledge and policies throughout Europe. The eucen vision is a European model of lifelong learning based on democracy, equal rights and social justice (see for instance, COMMIT project, 539519-LLP-1-2013-1-BE-ERASMUS-ESIN) and contributing to economic, social and cultural needs (www.eucen.eu).

\section{4 eucen, a collaborative inter-organisational network}

This paragraph describes eucen by using the concept of network in an organisational perspective. Popp et al. (2013) notice that the phenomenon of a network is now commonly understood, but the concept is not always exactly defined or referred, distinctively from partnerships or strategic alliances. Therefore, based on previous works of Provan, Fish and Sydow (2007) and Weber and Khademian (2008), Popp et al. (2013) propose a working definition highlighting collaboration and cooperation and refer to network when enduring exchange relations are established between members and to collaborative inter-organizational network when three or more organisations are involved in the network and are working together toward a common purpose.

With its 178 members, mainly universities or national networks of universities, eucen is obviously an inter-organisational network.

But is it a collaborative one? To answer this question, we use the typology proposed by Popp et al. (2013) in their critical review of the literature regarding inter-organisational network applied in the public sector and more specifically to the health sector. Popp et al. (2013) identify ten distinctive types of network developing distinctive functions illustrating the cooperation and collaboration within the network. To our understanding, eucen fulfils at least the six following functions:

1. 'Information sharing' and diffusion: eucen disseminates its information via its social media (i. e. LinkedIn, Twitter, Facebook), via its web site and via the 'eucen Highlights' distributed once a month and the 'eucen Informs' newsletter published three times per year. Moreover, eucen's office facilitates communication, 
liaison and collaboration with other appropriate bodies and organisations active in lifelong learning or related subjects.

2. 'Knowledge generation' and exchange: eucen writes European projects proposals in order to develop new knowledge, to identify new ideas, new framework and practices amongst project partners and to spread them to eucen members and beyond.

3. 'Developmental': beside the information exchange, eucen organises a specific event 'the autumn seminar' once a year, dedicated to professional development of member's teams in order to enhance their abilities to implement solutions and to solve problems. eucen provides opportunities for LLL staff development through staff exchange and seeks opportunities for LLL student exchange.

4. 'Learning' at individual, organisational, network and community level: eucen tries to turn any exchange, contact, or activity into a learning opportunity. The best example is probably the annual conference. But projects are also considered by partners as a learning process, as a discursive process where they learn from each other and adopt new position (see for instance the DIALOGUE project final publication in de Viron 2014).

5. 'Innovation': this function is closely connected with the 'knowledge generation' one. Via its involvement in European projects, eucen initiates or conducts research into lifelong learning. eucen also support members wishing to develop European LLL research. Moreover, when organising events, eucen promotes innovation by developing and organising creative spaces where diversity and openness are at work, inviting partners or participants to create new concepts or solutions.

6. 'Policy': as a main goal, eucen intends to represent the interests of the university lifelong learning community within higher education and to European policymakers. For example, eucen is involved in the LLL Platform and is one of the six VET providers Associations in the advice board for DG Employment, Social Affairs and Inclusion. eucen has recently set up an activity named 'eucen Policy Talks' dedicated to EU Policy makers and European LLL stakeholders, illustrating this function to contribute to EC Policy development.

Furthermore, it seems to us that eucen network has two more functions, quality and professional identity, not present in typology of Popp et al. (2013).

7. 'Quality': eucen encourages high standards in all areas of lifelong learning and strives to harmonise levels of quality for lifelong learning among its members. eucen developed many projects dedicated to quality (as examples, EQUIPE and EQUIPE Plus projects, dedicated to European Quality in Individualised Pathways in Education).

8. 'Professional identity' and recognition: being actors in LLL development is a new profession within universities. eucen is a convivial space where these new professionals, managers, teachers, researchers working in ULLL could meet. Members and newcomers are pleased to meet each other, exchange their points of views, explain without fear their problems or difficulties in implementing 
ULLL. eucen is a forum of interchange, beneficial for newcomers or experts in

ULLL, fostering a sense of belonging to a professional community.

Regarding these elements, we could certainly conclude that eucen is a collaborative inter-organisational network.

What are the potential benefits of inter-organisational networks?

Using again the framework developed by Popp et al. (2013) for the inter-organisational networks benefits, we would highlight at least three, i. e. learning, positive deviance and advocacy. We prioritise them as follows:

- Learning and capacity building are certainly the major ones, not only at the network level, but probably in the broader community of lifelong learning. Closely connected to learning benefit, innovation is also expanded via activities developed by the network.

- Positive deviance: the network provides a forum to think and act beyond the universities' norm, structure and mandate. As mentioned by Popp et al. (2013), members are able to work deliberately in deviation from their standard organisational processes, imagine new ways of thinking or doing and influence changes in current home systems. This benefit refers to the 'innovation' and 'professional identity' functions.

- Advocacy: the network legitimates positions and opinions. As outlined by Popp et al. $(2013$, p. 18) "it exerts more pressure due to greater political clout and community reach resulting from greater numbers of actors and diversity of network members (Provan/Lemaire 2012)". eucen network is legitimated to interact at European level, making recommendations to European institutions.

We would like to deepen the way the most important benefit of the network - i.e. learning and capacity building - expands and grows within eucen.

\section{Learning: eucen as a Community of Practice}

To examine and present the learning phenomena within eucen, we apply a community of practice framework (Lave/Wenger 1991; Wenger 2010).

A community of practice -in short CoP- is a group of people (usually individuals) who share a concern or a passion for something they do (the practice) and who learn how to do it better by interacting regularly (Wenger 1998).

Three fundamental items characterise a CoP: the domain, the community and the practice.

The domain characterises the relevance, the value and the purpose of the CoP. Internally, the members of the CoP agreed on the domain and are committed to it. In eucen case, the domain is University Lifelong Learning (ULLL), in all its forms and implementation. 
The community is the social construct where the CoP members interact and build relationships, engage in joint activities and help each other. It usually requires mutual respect, common identity, willingness to share and to interact regularly. In our case, interactions and relationships take place during the events organised by eucen, but also virtually via mail exchanges, virtual meetings, or bilateral meetings. "A community of practice can be viewed as a social learning system" (Wenger 2010, p. 179).

The practice is a crucial characteristic as a $\mathrm{CoP}$ is not a community of interest. CoP members are practitioners. They develop a shared repertoire of resources: lessons learned, tools used, ways of addressing recurring problems, documents and books and so on. eucen members are universities, i.e. organisations, but their representatives within the network are practitioners. Current representatives are (a) rector/president, vice rector/president or dean, dealing with ULLL strategies, (b) ULLL units' managers or staff members developing provisions (c) ULLL teachers or professors and (d) researchers in ULLL or adult education.

Within eucen, we observe professionals committed to taking part in learning together, sharing a common culture constantly engaged in sharing experience, exchanging information and knowledge, formally (during conference for instance via presentations, posters) and informally (during the 'open forum' organised during conference or discussions at breaks) and contributing to a common understanding and to collective knowledge.

To our point of view, eucen acts as a community of practice or is a CoP as suggested by Borzillo, Probst and Rainsch (2008), who assimilate a specific form of interorganizational network and community of practice.

During 25 years, eucen developed knowledge and expertise. We could affirm that a collective and common knowledge exists today. But this knowledge is largely diffused in the members' heads and only partially structured and stored in archives and files (mainly the web site). A future challenge for this community of practice would be to organise a repository to collect, maintain and diffuse this collective knowledge and make it more explicit and visible. In this process, another challenge will be to invite the national networks as specific members of the CoP to contribute and to integrate their own collective knowledge.

What are the main benefits of CoP?

As mentioned by Wenger (Wenger 1998, p. 4), "CoP have the potential to play a vital role in supporting both formal and informal staff development because they bring together groups of people with mutually shared interests and different degrees of knowledge and experience to develop their knowledge and understanding".

Moreover, as outlined by Jimenez-Silva and Olson (2012, p. 335), "As members of a community of practice interact, share and participate in a particular cultural practice, over time, they develop their understanding about the practice, about who they are, and about what they know in relation to the community and its goals". This phenom- 
enon certainly contributes to the development of function (7) 'professional identity' of eucen network.

Beyond the benefits, it is important to outline some prerequisites to develop effective learning within eucen as a CoP, i. e. the quality of the exchange content, the trust and the mutual respect of the members, the need for a culture of professional development, and the need for a language to mediate thinking about practice and to foster the dialogue between ULLL practitioners and ULLL researchers. The two first prerequisites are very frequently mentioned, see for instance Wenger (2010). The last two are highlighted by Blanton and Stylianou (2009) in their study on faculty professional development at university.

\section{6 eucen and national networks}

As mentioned before, amongst eucen members there are now 17 national networks from 15 countries. Learning together, exchanging good practices, influencing the national or regional environment are the main activities of these national networks. These networks are crucial relay for eucen as they are representative of a country or a region, understanding the legal framework and identifying the main challenges. They play a catalyst role within eucen.

All characteristics and benefits of networks or CoP presented in this chapter, could be applied to national networks dedicated to ULLL. From our point of view, national networks are useful instruments fulfilling at least the following functions amongst those described in paragraph 4 :

- 'Information sharing' and diffusion: national networks are active actors in information transmission, in a bi-directional way: from national context to European one and vice-versa. They also could disseminate information largely in their country, to higher education institutions, members or not-members of eucen.

- 'Quality': a national network is a useful instrument in quality development within a country, helping all, small and large institutions to implement LLL.

- 'Innovation' and 'Policy': national networks are the place to carry out or implement organisational changes, through collegial forms of cooperation, taking into account the specific legal context.

The German Association for University Continuing and Distance Education, DGWF, is one of the leading national networks within eucen. DGWF is one of the first national networks, created in 1970, but also one of the largest networks with its more than 320 institutional and individual members. It is worthwhile to mention that German universities and their network had a special role in eucen's creation as the German network existed before and as the first elected president of eucen was Prof. Peter von Mitschke-Collande from Leibniz Universität Hannover. Other German universities' representatives have been also active within eucen's Steering Committee during these last years, such as Dr. Beate Hoerr from Johannes Gutenberg University of Mainz or Mr. Helmut Vogt, Akademischer Direktor at University of Hamburg. 
As an illustration, we apply the framework of inter-organisational network functions developed by Popp et al. (2013) to DGWF. Starting from the activities organised by the network as described on their website (DGWF 2016 https://dgwf.net/ueberuns/), we establish the following correspondence between activities and functions of the network:

- Publications and documentation: in particular, the magazine 'HOCHSCHULE UND WEITERBILDUNG' published twice a year is a nice illustration of function (1) 'Information sharing'

- Annual meetings, conferences, workshops, conferences and symposia contribute largely to functions (3) 'Developmental' and (4) 'Learning'. The diversity of DGWF training provision and the quality of the continuing training data bases have to be emphasised.

- Advising institutions and individuals

- Recommendations and opinions: those activities contribute to functions (6) 'Policy', trying to impact the legal or institutional context, (7) 'Quality' of the lifelong and distance learning and (8) 'Professional identity' of the actors involved in LLL and distance learning.

- Scientifically based research and research projects promoted or supported by the network fulfil the function (2) 'Knowledge generation' and (5) 'Innovation' as "Lifelong learning requires educational institutions to make a change of perspective ... [putting] biographical history learning processes of individuals in focus" (DGWF, annual conference, 2016).

At first glance, DGWF appears to be a collaborative inter-organisational network fulfilling the eight functions.

\section{Perspective for international and national networks: moving to learning organisations?}

We notice that a key function and benefit of inter-organisational networks, being national or international, is learning in an environment that is closely linked to knowledge creation and innovation.

\section{How could networks improve this function in the future?}

As mentioned before in the case of eucen, the development of a systematic knowledge repository is certainly a first step for improving the learning process within inter-organisational networks.

But we could do more. The learning process could be generalised moving from 'CoP learning' to 'organisational learning' that links expansion of learning, gains and creation of knowledge with improved performance (Senge 1993; Easterby-Smith/Lyles 2005; Knight/Pye 2005; Popp et al. 2013). From an organisational learning perspective, Popp et al. (2013, p. 34) suggest that "inter-organisational networks focused on 
learning as a critical function may well consider the value of developing internal 'dynamic capability' and/or 'absorptive capacity'", in other words, to systemise learning in order to develop network capabilities to learn from inside, from all members or groups of members, from its past and current experience, but also to learn from outside the network, stakeholders or external actors.

Burley et al. (2012) in their study on the role of consortia developing the needed cultural and strategic shifts for a new delivery of higher educational content, suggest that the power of consortia -in our case network- to extend, expand, and exploit this learning capacity, to deploy organizational learning may represent a vast, untapped resource.

We could say that networks have the potential to become learning organisations, enabling their members to cope with changes and challenges mentioned in the introduction.

This organisational learning perspective could not take place without involving all ULLL stakeholders and mainly the lifelong learners. They are the final beneficiaries of the ULLL and the final purpose of this evolution is to improve their autonomy in learning and their capacity to act in a rapidly changing world and challenging environment.

\section{Bibliography}

Blanton, M./Stylianou, D. A. (2009): Interpreting a Community of Practice Perspective in Discipline-Specific Professional Development. In: Higher Education, Innovation in Higher Education 34, pp. 79-92.

Borzillo, S./Probst, G./Raisch, S. (2008): The Governance Paradox: Balancing Autonomy and Control in Managing Communities of Practice. In: Academy of Management Proceedings, 2008(1), pp. 1-6.

Burley, D./Gnam, C./Newman, R./Straker, H./Babies, T. (2012): Leveraging higher education consortia for institutional advancement. International Journal of Educational Management, 26(3), pp. 274-283.

Davies, P. (2007): The Bologna process and University Lifelong Learning: the state of play and future Directions', BEFLEX Project, EUCEN publication, November 2007, Spain. http://www.eucen.eu/BeFlex/index.html

Davies, P. (2010): Lifelong Learning Universities: developing Flexibility and Sustainability. BEFLEX Plus Training Pack, BEFLEX Plus Project, EUCEN publication, January 2010, Spain. http://www.eucen.eu/BeFlex\%2oPlus

de Viron, F. (2014): on behalf of the DIALOGUE consortium (Ed.): Bridges between Research and Practice in University Lifelong Learning: Policy Report and Recommendations. Barcelona. (C) The DIALOGUE consortium, 2014. http://dialogue.eucen.eu 
de Viron, F./Davies, P. (2015): From University Lifelong Learning to Lifelong Learning Universities - developing and implementing effective strategy, in The Role of Higher Education in Promoting Lifelong Learning. In: Yang, J./Schneller, Chr./Roche, St. (Ed.): UNESCO Institute for Lifelong Learning, Publication Series on Lifelong Learning Policies and Strategies: No. 3.

DGWF (2016): Deutsche Gesellschaft für wissenschaftliche Weiterbildung und Fernstudium. https://dgwf.net/ueber-uns/ [Accessed: 21.06.2016]

Easterby-Smith, M./Lyles, M.A. (2005): Handbook of Organizational Learning and Knowledge Management, edited by Mark Easterby-Smith and M.A. Lyles, Blackwell Publishing.

Knight, L./Pye, A. (2005): Network learning: An empirically-derived model of learning by groups of organizations. In: Human Relations, 58(3), pp. 369-392.

Jimenez-Silva, M./Olson, K. A. (2012): Community of Practice in Teacher Education: Insights and Perceptions. In: International Journal of Teaching and Learning in Higher Education, 24(3), pp. 335-348.

Lave, J./Wenger, E. (1991): Situated Learning: Legitimate Peripheral Participation. Cambridge: Cambridge University Press.

Popp, J./MacKean, G./Casebeer, A./Milward, H. B./Lindstrom, R. (2013): Inter-organizational networks: A critical review of the literature to Inform practice. http://www.re search4children.com/data/documents/NetworkLiReview-Feb27-2013-Final.pdf

Provan, K. G./Fish, A./Sydow, J. (2007): Inter-organizational networks at the network level: A review of the empirical literature on whole networks. In: Journal of Management, 33(3), p. 479.

Provan, K. G./Lemaire, R. H. (2012): Core concepts and key ideas for understanding public sector organizational networks: Using research to inform scholarship and practice. In: Public Administration Review, 72(5), pp. 638-648.

Senge, P. M. (1993): The fifth discipline: The Art and Practice of the Learning Organisation, Random House Business Books.

Sursock, A./Smidt, H. (2010): Trends 2010: A Decade of Change in European Higher Education. Brussels, European University Association. http://www.eua.be/publications/ eua-reports-studies-and-occasional-papers.aspx

Sursock, A. (2015): Trends 2015: Learning and Teaching in European Universities. Brussels, European University Association. http://eua.be/Libraries/higher-education/ trends-2015.pdf?sfvrsn=0

Weber, E. P./Khademian, A. M. (2008): Wicked problems, knowledge challenges, and collaborative capacity builders in network settings. Public Administration Review, 68(2), pp. 334-349.

Wenger, E. (1998): Communities of Practice: Learning, Meaning, and Identity, New York: Cambridge University Press.

Wenger, E. (2010): Communities of practice and social learning systems: the career of a concept. In: Social learning systems and communities of practice, Springer London, pp. 179-198. 
Perspektiven auf

Hochschulweiterbildung 



\section{Rechtliche und organisatorische Herausforderungen bei der Implemen- tierung der wissenschaftlichen Weiterbildung}

URSULA BADE-BECKER

Der Bedeutungsgewinn wissenschaftlicher Weiterbildung in der bildungspolitischen Diskussion insbesondere vor dem Hintergrund zunehmender Wissensdynamisierung, der demografischen Entwicklung und dem Umbau der Hochschulen zu Institutionen lebenslangen Lernens ist unübersehbar (Wolter 2011), zudem ist wissenschaftliche Weiterbildung seit der Novellierung des Hochschulrahmengesetzes im Jahre 1998 Kernaufgabe der Hochschulen neben Forschung und Lehre (HRG 『2 Abs. 1).

Dennoch wird die Aufgabe der wissenschaftlichen Weiterbildung an Hochschulen nicht von förderlichen Rahmenbedingungen begleitet (Hanft et al. 2016, S. 247 f.).

In diesem Beitrag sollen einige rechtliche und organisatorische Herausforderungen im Kontext der strukturellen Implementierung von wissenschaftlicher Weiterbildung an staatlichen Hochschulen schlaglichtartig beleuchtet werden.

\section{Organisation und organisatorische Verankerung}

Die Hochschulgesetze der Länder lassen die organisatorisch-institutionelle Verortung der wissenschaftlichen Weiterbildung weitgehend offen. Infolgedessen haben sich an den Hochschulen je nach Zielsetzungen und Ressourcen zahlreiche Organisationsvarianten entwickelt. Hochschulintern besteht die Möglichkeit einer zentralen Organisation in Form von wissenschaftlichen Einrichtungen, zentralen Betriebseinheiten, als Aufgabe eines zuständigen Dezernats, als Aufgabe von Rektoratsbeauftragten, als Stabsstelle oder als eine dezentrale Organisation (Fakultäten, Fachbereiche) und als Kombination zentraler und dezentraler Organisationsformen. 
Daneben wird die wissenschaftliche Weiterbildung aus unterschiedlichen, meist finanziell und administrativ motivierten Gründen zunehmend auch hochschulextern organisiert. In diesen Fällen werden eigens Vereine, Gesellschaften mit beschränkter Haftung (GmbHs; z. T. in gemeinnütziger Variante), Akademien (z. T. in kooperativer Form mehrerer Hochschulen) oder Aktiengesellschaften genutzt, um in Addition zur institutionell in der Hochschule selbst verankerten Weiterbildung neue Spielräume für die Realisierung der Weiterbildung zu schaffen. Welche Organisationsform gewählt wird, hängt nicht nur von rechtlichen Rahmenbedingungen und dem jeweiligen Profil der Hochschule ab, sondern auch von den Zielen, die mit der jeweiligen Option erreichbar sind.

Mit der Wahl einer Organisationsform sind jeweils eine Vielzahl von Implikationen verbunden, z. B. hinsichtlich der Steuerbarkeit (Umsatz-, Körperschaft- und Gewerbesteuerpflicht etc.), des Personalrechts und des Trennungsrechts. Bei externer Organisation kommen rechtsformspezifische Anforderungen hinzu.

Wissenschaftliche Weiterbildung ist eher selten in den Fakultäten direkt verankert, soll jedoch wissenschaftsbezogen und fachwissenschaftlich verantwortet sein. Aufgrund der Forderung nach (Voll-)Kostendeckung für wissenschaftliche Weiterbildung, die in allen Hochschulgesetzen aufgenommen ist, hat sich die Weiterbildung zudem den Prinzipien des Marktes zu stellen mit wettbewerblichem Handeln und starker Kunden- und Serviceorientierung. Diese am Markt orientierte unternehmerische Kultur steht jedoch im Widerspruch zur regelgeprägten Ordnung der Verwaltung wie auch zur disziplinär geprägten Logik der Wissenschaft in den Hochschulen (Hanft et al. 2016, S.31 f.).

Exkurs: Organisationstheoretisch betrachtet gelten Hochschulen als hybride Organisationen mit funktionalen, divisionalen und netzwerkförmigen Strukturen, die nebeneinander bestehen (Hanft et al. 2016, S. 30 ff.) und dennoch lose gekoppelt sind. So trifft die bürokratische Ordnung der Verwaltung auf disziplinär geprägte Kulturen der Wissenschaft. Rechtliche Rahmenvorgaben, Anweisungen und Hierarchie prägen die Verwaltung, fachdisziplinäre Orientierungen, Selbstbestimmung und Autonomie die Wissenschaft. Vermittelnde Steuerungsstrukturen fehlen weitgehend.

Wissenschaftliche Weiterbildung lässt sich weder eindeutig der Verwaltung noch der Wissenschaft zuordnen, obgleich sie Elemente beider Teilbereiche aufnimmt (ebd., S. 31). Aus organisationaler Sicht sieht Wilkesmann (2007, S. 3; 2010, S. 32 f.) die wissenschaftliche Weiterbildung ebenfalls nicht im Kern der Hochschule angesiedelt, obgleich sie zu den Kernaufgaben zählt, sondern eher am Rande, an der Grenze zwischen der Organisation Universität und der Umwelt mit ihren jeweils unterschiedlichen Rationalitäten (Kloke/Krücken 2010, S.38).

1 Nach Luhmann (1964, S. 224) interpretieren die Grenzstellen „die Umwelt für das System. Sie müssen Umweltinformationen sichten und sieben und sie in eine Sprache bringen, die im System verstanden und akzeptiert wird“. 


\section{Reorganisation}

Derzeit sind zahlreiche (Re-)Organisationsprozesse im Bereich wissenschaftlicher Weiterbildung in den Hochschulen zu beobachten, die insbesondere vor dem Hintergrund von Entwicklungs- und Profilierungserfordernissen der Hochschulen im Zuge von neuen Steuerungsmodellen in der Verwaltung und in der Wissenschaft zu sehen sind. Hinzu kommt der Bedeutungszuwachs der Hochschulen im Rahmen von regionalen Entwicklungsstrategien, die auch die wissenschaftliche Weiterbildung und ihre Einrichtungen berühren (Dollhausen/Ludwig/Wolter 2013; Wolter/ Banscherus 2015).

$\mathrm{Zu}$ beobachten ist insgesamt „eine (Re)Zentralisierung von Aufgaben und Funktionen der strategischen Planung und Steuerung der Hochschulentwicklung, insbesondere eine deutliche Stärkung des Hochschulmanagements“ (Dollhausen/Ludwig/ Wolter 2013, S.10). Ein Königsweg kann bei den Reorganisationsmaßnahmen der wissenschaftlichen Weiterbildung nicht ausgemacht werden. Die Maßnahmen hängen von den bisherigen organisatorischen Verankerungen ab, von bereits vorhandenen Entwicklungspfaden sowie - neben rechtlichen Bestimmungen - von den künftigen Aufgaben und Organisationszielen.

Der wissenschaftlichen Weiterbildung wird auch eine noch unausgereifte Position inner- und außerhalb der Hochschule bescheinigt, der eine gewisse Entwicklungszeit zugestanden werden muss, ehe sie als vollwertiger Teilbereich der Hochschule neben Forschung und Lehre anerkannt werden kann (Franz/Feld 2014).

\section{Wettbewerbsrecht}

Bereits im Rechtsgutachten von Mestmäcker/Veelken (1990) wurde geprüft, inwieweit das Wettbewerbsrecht auf das Angebot wissenschaftlicher Weiterbildung durch die staatlichen Hochschulen anzuwenden ist. Die Antwort war eindeutig: „Es besteht Konsens darüber, dass die Hochschulen nur wissenschaftliche Weiterbildung anbieten dürfen (Grundsatz der Profiltreue oder Anknüpfung an andere Hochschulaufgaben). Eine Konkurrenzsituation besteht mithin auch nur zu anderen Anbietern wissenschaftlicher Weiterbildung“ (vgl. Mestmäcker/Veelken 1990, S.8). Weiterhin heißt es: „Soweit es sich bei den Angeboten wissenschaftlicher Weiterbildung um ein marktfähiges Gut handelt, nehmen die Hochschulen mit dem Angebot wissenschaftlicher Weiterbildung mithin am allgemeinen Wirtschaftsverkehr teil“ (ebd., S. 51). Die Preisgestaltung ist betriebswirtschaftlich zu begründen.

\section{Europäisches Beihilferecht}

Nachhaltige Auswirkungen auf Finanzierung und Organisation wissenschaftlicher Weiterbildung sind mit dem Europäischen Beihilferecht verbunden, mit dem sich die staatlichen Hochschulen seit 2007 auseinandersetzen müssen.

Im Vertrag über die Arbeitsweise der Europäischen Union (AEUV, 2012) wird in Art.107 Abs.1 der Beihilfebegriff grundlegend behandelt: „Soweit in den Verträgen 
nicht etwas anderes bestimmt ist, sind staatliche oder aus staatlichen Mitteln gewährte Beihilfen gleich welcher Art, die durch die Begünstigung bestimmter Unternehmen oder Produktionszweige den Wettbewerb verfälschen oder zu verfälschen drohen, mit dem Binnenmarkt unvereinbar, soweit sie den Handel zwischen Mitgliedstaaten beeinträchtigen.

Waren staatliche Hochschulen bis Ende 2006 nicht vom Europäischen Beihilferecht betroffen, so änderte sich dieses mit dem Inkrafttreten des EU-Gemeinschaftsrahmens für staatliche Beihilfen für Forschung, Entwicklung und Innovation ab dem 01.01.2007, der nach seiner Reformierung durch den „Unionsrahmen für staatliche Beihilfen zur Förderung von Forschung, Entwicklung und Innovation“ abgelöst wurde und seit 01.07.2014 in Kraft ist. Ziel des aktuellen Unionsrahmens wie auch des vorherigen Gemeinschaftsrahmens ist die Sicherstellung eines Wettbewerbs, der nicht durch unzulässige Gewährung von staatlichen Zuwendungen verzerrt wird.

Das Beihilferecht erfasst die wirtschaftlichen Tätigkeiten einer staatlichen Hochschule (Tauer/Göbel 2014, S. 21). Da geeignete Kriterien für eine Unterscheidung von wirtschaftlichen und nichtwirtschaftlichen Tätigkeiten im Unionsrahmen nicht detailliert bzw. abschließend definiert sind, legte die Kultusministerkonferenz (KMK) 2012 ein entsprechendes Analyseraster vor, an dem sich die Hochschulen bei ihrer Bewertung und Einordnung orientieren können.

Hinsichtlich der wissenschaftlichen Fort- und Weiterbildung an (staatlichen) Hochschulen wird konzediert, dass deren Einordnung als wirtschaftliche Tätigkeit differenziert gesehen wird. „Grundsätzlich ist dort, wo Angebote sich in Konkurrenz zu anderen Angeboten, insbesondere von privaten Anbietern befinden, von einem Markt und damit einer wirtschaftlichen Tätigkeit auszugehen.

Insbesondere deswegen, weil die Zuordnung der Weiterbildung als gesetzliche Aufgabe der Hochschulen im deutschen, nicht aber im europäischen Recht durchgehend vorgesehen ist, wird den Hochschulen seitens der Wirtschaftsprüfer geraten, die Weiterbildung grundsätzlich als wirtschaftliche Tätigkeit auszuweisen (...). Dies korrespondiert auch mit der Regelung einiger Hochschulgesetze und der Forderung von Landesrechnungshöfen, die Weiterbildung grundsätzlich kostendeckend bzw. auf privatwirtschaftlicher Grundlage durchzuführen.

Je enger das Angebot sich aus dem spezifischen Bildungsauftrag der Hochschulen ableiten lässt, in dessen Vordergrund die Ausbildung von mehr und besser qualifizierten Humanressourcen (nichtwirtschaftliche Tätigkeit) steht, desto eher kann die Einordnung als nichtwirtschaftliche Tätigkeit zutreffen, Beispiel: duale Studiengänge“ (KMK 2012, S. 5 f.). Ein abschließender Katalog zur rechtlich eindeutigen Abgrenzung wird explizit nicht vorgelegt, dieser ist jeweils nach Prüfung für und durch die jeweilige Hochschule selbst zu erstellen.

Beide Geschäftsfelder der Hochschulen (nicht-wirtschaftliche und wirtschaftliche Tätigkeiten) müssen bezüglich Kosten und Finanzierung eindeutig voneinander 
getrennt geführt werden, sodass nachgewiesen werden kann, dass eine öffentliche Quersubventionierung wirtschaftlicher Tätigkeiten nicht erfolgt.

Wissenschaftliche Weiterbildungen von Hochschulen, die als wirtschaftlich eingestuft und innerhalb der Hochschule organisiert und durchgeführt werden, sind in der (staatlichen) Hochschule als „Betrieb gewerblicher Art“ (BgA) zu führen. Rechtliche Grundlage für das Besteuerungssubjekt $\mathrm{BgA}$ bildet das Körperschaftssteuergesetz (KStG).

Die Empfehlung der grundsätzlichen Einstufung von wissenschaftlicher Weiterbildung als wirtschaftliche Tätigkeit und der Einordnung als nichtwirtschaftliche Tätigkeit bei enger Ableitung aus dem spezifischen Bildungsauftrag im speziellen Fall hat zur Folge, dass Hochschulen bei der beihilferechtlichen Beurteilung ihrer Weiterbildungsangebote zumeist Gutachter heranziehen (müssen), um ungewollte Rechtsfolgen zu vermeiden. Ein mögliches Vorgehen wird bspw. im Rechtsgutachten der Universität Freiburg i. Brg. (2014) beschrieben.

\section{Vollkostenrechnung}

Neben der beihilferechtlichen Beurteilung stellt sich im Weiteren die Frage nach den anzusetzenden Kosten einer Weiterbildung im Sinne einer Vollkostenrechnung. Komponenten und Berechnungsgrundlagen sind keinesfalls eindeutig und möglicherweise von Hochschule zu Hochschule (und von Bundesland zu Bundesland) unterschiedlich. Hinzu kommen Fragen der Vorfinanzierung von neuen Weiterbildungsangeboten und dem Umgang mit möglichen Defiziten.

\section{Finanzierung und Personal}

\section{Grundfinanzierung}

Bereits in ihrem Positionspapier zur wissenschaftlichen Weiterbildung, das sich an Politik und Hochschulleitungen richtete und Empfehlungen für einen Ausbau der wissenschaftlichen Weiterbildung gab, sprach sich die Hochschulrektorenkonferenz (2008, S. 6 f.) für ein finanzielles Commitment der Länder zur Finanzierung des Weiterbildungsbereichs aus: „Wollen Hochschulen nicht nur personen- oder fakultätsspezifische Veranstaltungen in der Weiterbildung anbieten, sondern sich als Hochschule insgesamt des Themas annehmen, auch Studiengänge anbieten und ein längerfristig angelegtes Gesamtprogramm entwickeln, so muss ihre Grundfinanzierung Mittel für eine entsprechende Infrastruktur umfassen."

Dass diese Forderung nicht an Aktualität eingebüßt hat, zeigt auch eine der Handlungsempfehlungen, die als Ergebnis der Fachkonferenz „Akademische Weiterbildung - eine Zukunftsaufgabe für Hochschulen!“ der Friedrich-Ebert-Stiftung im Oktober 2015 veröffentlicht wurde (Borgwardt 2016, S. 12): „Entscheidend ist jedoch, dass die Hochschulen auch strukturell - durch eine geeignete Ausstattung und aus- 
reichend Personal - in die Lage versetzt werden, kompetente akademische Weiterbildungsträger zu sein. Sie brauchen dafür eine angemessene und nachhaltig gesicherte staatliche Grundfinanzierung, damit sie ihre öffentliche Verantwortung im Bereich Weiterbildung wahrnehmen und eine kostendeckende Finanzierung der Angebote erreichen können."

Sofern einzelne Hochschulen diese Grundfinanzierung übernehmen, so bleibt die Frage nach der beihilferechtlichen Beurteilung derselben.

\section{Personal}

In den Angeboten wissenschaftlicher Weiterbildung kommen in der Lehre zumeist wissenschaftlich ausgewiesenes Personal der eigenen und anderer Hochschulen zum Einsatz sowie - meist in geringerem Umfang - qualifizierte Praktikerinnen und Praktiker. Je nachdem, wie die Weiterbildungsmaßnahme organisatorisch verortet ist, werden die Lehrleistungen im Nebenamt, in Nebentätigkeit, über Lehr- oder Honoraraufträge sowie ggf. über Anrechnung auf das Deputat vergütet.

Organisatorisch, verwaltend und technisch tätiges Personal wird jedoch eher weniger aus dem Stammpersonal der Hochschulen rekrutiert, was zur Folge hat, das nicht nur jeweils adäquat qualifiziertes Personal angeworben werden muss - ggf. auch unter Berücksichtigung einschlägiger Fristen und Verfahren -, sondern dass dieses i.d. R. auch in Abhängigkeit von den finanziellen Ressourcen (z. B. aus Förderprojekten oder erwarteten Einnahmen aus Teilnahmeentgelten) jeweils nur mit befristeten (Teil-)Zeitverträgen beschäftigt werden kann. Projektförmige, befristete, oftmals prekäre Beschäftigungsverhältnisse stehen einer Nachhaltigkeit von wissenschaftlichen Weiterbildungsangeboten jedoch entgegen. Die Personalfluktuation wird erhöht, erhebliche Wissens- und Kompetenzverluste können die Folge sein (Hanft 2014).

Mit der Novellierung des Wissenschaftszeitvertragsgesetzes (2016), die am 17.03.2016 in Kraft trat, verschärfte sich die Situation nochmals. Das Wissenschaftszeitvertragsgesetz (WissZeitVG) regelt, wie die Arbeitsverträge für das wissenschaftliche und künstlerische Personal an staatlichen Hochschulen zeitlich befristet werden können. Die Novellierung zielt insbesondere darauf ab, unsachgemäßen Kurzzeitbefristungen von wissenschaftlichem und künstlerischem Personal entgegenzuwirken. Waren zuvor im WissZeitVG auch die befristeten Beschäftigungen des nicht-wissenschaftlichen Personals geregelt, so gilt für diese Gruppe zukünftig das Teilzeit- und Befristungsgesetz (TzBfG) mit höheren Anforderungen hinsichtlich des Befristungsgrundes und für den Abschluss mehrerer befristeter Arbeitsverhältnisse hintereinander (DFG 2016).

Kreutz, Wanken und Meyer (2012, S. 139) bemerken: „Die Etablierung der weiterbildenden Angebotsstruktur deutscher Hochschulen vollzog sich bisher weitestgehend durch Pilotprojekte, die eine nachhaltige Implementierung i.d. R. nicht gewährleisten können. Eine fortwährende Etablierung setzt jedoch langfristige finanzielle Absi- 
cherung voraus, denn die Aufwendungen im Bereich der wissenschaftlichen Weiterbildung sind nur durch langfristige Planungs- und Organisationsstrukturen und durch stabile Beschäftigungsverhältnisse zu ermöglichen.“

\section{Schlussbemerkung}

Mit dem Umbau der Hochschulen zu Institutionen des lebenslangen Lernens geht die Forderung nach dem Ausbau der wissenschaftlichen Weiterbildung an den staatlichen Hochschulen einher.

Dieses kann nur gelingen, wenn die wissenschaftliche Weiterbildung von verlässlichen, länderübergreifend vergleichbaren Rahmenbedingungen begleitet wird und die langfristige organisationale Einbindung in die Hochschulstrukturen gesichert ist. Insbesondere erscheint es notwendig, die massive Verunsicherung vieler Hochschulen infolge der Veränderungen im Rahmen des EU-Beilhilferechts aufzulösen.

\section{Literatur}

Albert-Ludwigs-Universität Freiburg in Kooperation mit Fraunhofer (2014): Freiräume für wissenschaftliche Weiterbildung. Rechtsgutachten. Vorgaben für die Preisgestaltung der wissenschaftlichen Weiterbildung an der Universität Freiburg unter besonderer Berücksichtigung des europäischen Beihilferechts. Stand Januar 2014. Malte Marwedel, Ass. iur., Universität Freiburg. Eine Veröffentlichung des Z-Projektes. Im April 2014, Freiburg i. Brg. https://www.offenehochschule.uni-freiburg.de/data/doc/zprojekt_pdf/ rechtsgutachten [Zugriff: 16.06.2016]

Borgwardt, A. (Hrsg.) (2016): Akademische Weiterbildung. Friedrich-Ebert-Stiftung, Schriftenreihe Hochschulpolitik, Bonn. http://library.fes.de/pdf-files/studienfoerde rung/12365-20160317.pdf [Zugriff: 26.06.2016]

Deutsche Forschungsgemeinschaft (DFG) (2016): Handreichung zum Wissenschaftszeitvertragsgesetz (WissZeitVG). http://dfg.de/download/pdf/foerderung/rechtliche_rah menbedingungen/handreichung_wisszeitvg.pdf [Zugriff: 23.06.2016]

Dollhausen, K./Ludwig, J./Wolter, A. (2013): Organisation und Re-Organisation wissenschaftlicher Weiterbildung in einer bewegten Hochschullandschaft. In: Hochschule und Weiterbildung 2013(2), S. 10-13.

Europäische Kommission (2014): Mitteilungen der Kommission. Unionsrahmen für staatliche Beihilfen zur Förderung von Forschung, Entwicklung und Innovation (2014/C 198/01). Amtsblatt der Europäischen Union C 198/1, 27.06.2014. 
Franz, M./Feld, T. (2014): Steuerungsproblematiken im Prozess der Implementierung wissenschaftlicher Weiterbildung. In: REPORT Zeitschrift für Weiterbildungsforschung. Opening Higher Education to Adult Learners - Concepts and Research Results, 2014(4), S. 28-40, Bielefeld.

Hanft, A./Brinkmann, K./Kretschmer, S./Maschwitz, A./Stöter, J. (2016): Organisation und Management von Weiterbildung und Lebenslangem Lernen an Hochschulen. Ergebnisse der wissenschaftlichen Begleitung des Bund-Länder-Wettbewerbs Aufstieg durch Bildung: offene Hochschulen. Band 2. Münster, New York: Waxmann. https://de.of fene-hochschulen.de/wb2011-2015/teilstudien/ [Zugriff: 16.06.2016]

Hanft, A. (2014): Management von Studium, Lehre und Weiterbildung an Hochschulen. Studienreihe Bildungs- und Wissenschaftsmanagement. Band 13. Münster, New York: Waxmann.

Hochschulrahmengesetz (HRG) in der Fassung der Bekanntmachung vom 19. Januar 1999 (BGBl. I S. 18), zuletzt geändert durch Artikel 2 des Gesetzes vom 12. April 2007 (BGBl. I S. 506). https://www.gesetze-im-internet.de/bundesrecht/hrg/gesamt.pdf

Hochschulrektorenkonferenz (2008): HRK-Positionspapier zur wissenschaftlichen Weiterbildung. Beschluss des 588. Präsidiums am 7.7.2008. Bonn. Download über https:// www.hrk.de/themen/lehre/weiterbildung/ [Zugriff: 10.06.2016]

Hochschulrektorenkonferenz (2016): Wissenschaftliche Weiterbildung. https://www.hrk. de/themen/lehre/weiterbildung/ [Zugriff: 13.06.2016]

Kloke, K./Krücken, G. (2010): Grenzstellenmanager zwischen Wissenschaft und Wirtschaft? Eine Studie zu Mitarbeiterinnen und Mitarbeitern in Einrichtungen des Technologietransfers und der wissenschaftlichen Weiterbildung. In: Beiträge zur Hochschulforschung, 32(3), S. 32-52.

KMK (Kultusministerkonferenz) (2012): Analyseraster zur Unterscheidung wirtschaftlicher und nichtwirtschaftlicher Tätigkeit von Hochschulen. Ein Leitfaden. http://www.kmk. org/fileadmin/Dateien/pdf/Wissenschaft/SO_120928_AnalyserasterTrennungsrech nung.pdf [Zugriff: 10.06.2016]

Kommission: Gemeinschaftsrahmen für staatliche Beihilfen für Forschung, Entwicklung und Innovation (2006/C 323/01). Amtsblatt der Europäischen Union C323/1, 30.12.2006.

Körperschaftsteuergesetz (KStG) in der Fassung der Bekanntmachung vom 15.10.2002 (BGBl. I S. 4144), zuletzt geändert durch Gesetz vom 22.12.2014 (BGBl. I S.2417) m.W. v. 31.12.2014. https://dejure.org/gesetze/KStG [Zugriff: 01.08.2015]

Kreutz, M./Wanken, S./Meyer, R. (2012): Organisationsformen wissenschaftlicher Weiterbildung. In: Hessische Blätter für Volksbildung 2/2012 - 62. Jg. 2012 - Nr. 2, hgg. vom Hessischen Volkshochschulverband - hvv-Institut gGmbH, Frankfurt am Main.

Luhmann, N. (1964): Funktionen und Folgen formaler Organisation. Schriftenreihe der Hochschule Speyer, Bd. 20. Berlin: Duncker \& Humblot.

Mestmäcker, E.-J./Veelken, W. (1990): Wettbewerb in der Weiterbildung an Hochschulen. Die Anwendung des Wettbewerbsrechts auf das Angebot wissenschaftlicher Weiterbildung durch die staatlichen Hochschulen. - Rechtsgutachten - Reihe Bildung - Wissenschaft - Aktuell 2. Bonn: BMBW. 
Sekretariat der ständigen Konferenz der Kultusminister der Länder in der Bundesrepublik Deutschland: III C - 4120/6.1.2, Analyseraster zur Unterscheidung wirtschaftlicher und nichtwirtschaftlicher Tätigkeit von Hochschulen. Ein Leitfaden. (Stand: 28.09.2012) Quelle: http://www.kmk.org/fileadmin/pdf/Wissenschaft/SO_120928_ AnalyserasterTrennungsrechnung.pdf (22.06.2016)

Tauer, J./Göbel, S. (2014): Die Hochschulweiterbildung zwischen Gemeinwohl und Rechtssicherheit. In: DGWF Hochschule \& Weiterbildung 1/14, Bielefeld Juni 2014, S. 20-25.

Vertrag über die Arbeitsweise der Europäischen Union (Konsolidierte Fassung) (AEUV). Amtsblatt der Europäischen Union C 326/47, 26.10.2012. Quelle: http://eur-lex.eu ropa.eu/search.html?qid=1438509699228\&text=AEUV\&scope=EURLEX\&type=quick\& lang=de Datei: CELEX_12012E_TXT_DE_TXT.pdf [Zugriff: 01.08.2015]

Wilkesmann, U. (2010): Die vier Dilemmata der wissenschaftlichen Weiterbildung. In: ZSE Zeitschrift für Soziologie der Erziehung und Sozialisation, 30(1)1, S. 28-42.

Wilkesmann, U. (2007): Die Organisation der Weiterbildung. Discussion papers des Zentrums für Weiterbildung, Universität Dortmund, o8-2007. http://www.zfw.uni-dort mund.de/wilkesmann [Zugriff: 23.06.2016]

Wissenschaftszeitvertragsgesetz vom 12. April 2007 (BGBl. I S. 506), zuletzt geändert durch Artikel 1 des Gesetzes vom 11. März 2016 (BGBl. I S. 442). https://www.gesetzeim-internet.de/wisszeitvg/BJNR050610007.html [Zugriff: 23.06.2016]

Wolter, A. (2011): Die Entwicklung wissenschaftlicher Weiterbildung in Deutschland: Von der postgradualen Weiterbildung zum lebenslangen Lernen. In: Beiträge zur Hochschulforschung, 33(4), S. 8-35.

Wolter, A./Banscherus, U. (2015): Der Bund-Länder-Wettbewerb „Aufstieg durch Bildung: offene Hochschulen“ im Kontext der (inter-)nationalen Diskussion um lebenslanges Lernen. In: Klages, B./Bonillo, M./Reinders, S./Bohmeyer, A. (Hrsg.): Gestaltungsraum Hochschullehre. Potenziale nicht-traditionell Studierender nutzen. Opladen, Berlin, Toronto: Budrich UniPress Ltd., S. 17-35. 



\section{Offene Hochschule: Motor wissenschaftlicher Weiterbildung?}

ANDRÄ WOLTER

\section{Vorgeschichte}

Seit 2011 werden in Deutschland zahlreiche Hochschulen durch Fördermittel aus dem Bund-Länder-Wettbewerb Aufstieg durch Bildung: offene Hochschulen bei der Entwicklung und Erprobung von berufsbegleitenden, weiterbildenden und anderen flexiblen Studienangeboten unterstützt. Diese Angebote richten sich primär an Zielgruppen, die bereits über eine berufliche Qualifikation verfügen, entweder einen nicht-akademischen Berufsabschluss oder bereits einen Hochschulabschluss. Maßgeblich beeinflusst wurde der Wettbewerb durch den internationalen Diskurs über lebenslanges Lernen und dessen Implementation im Hochschulbereich (ausführlicher Überblick bei Wolter/Banscherus 2016). Wenn auch der „Quantensprung“ für die Weiterbildung an Hochschulen immer noch aussteht, so hat dieser Wettbewerb die Entwicklung der wissenschaftlichen Weiterbildung in Deutschland ein ganzes Stück weiter vorangebracht. Das gilt sowohl für die Vielzahl als auch für die Vielfalt der an den Hochschulen geförderten Projekte und Maßnahmen. Das hat sich spürbar auch in der Arbeit der DGWF selbst niedergeschlagen, ablesbar z. B. an den Mitgliederzahlen oder am Interesse an den Jahrestagungen. Neben positiven Einschätzungen des Wettbewerbs gibt es jedoch auch skeptische Stimmen, die das Programm für eine Fehlinvestition (z. B. wegen fehlender Nachhaltigkeit) oder Fehlsteuerung (in Richtung Ökonomisierung der Weiterbildung an Hochschulen) halten.

In den 1990er Jahren und um die Jahrtausendwende war zunächst der Eindruck unter den Protagonisten der wissenschaftlichen Weiterbildung weit verbreitet, dass sich die Entwicklung der Weiterbildung an den Hochschulen in Deutschland an der Diskrepanz zwischen allgemeiner rhetorischer Hochschätzung und faktischer Beharrung festgefahren hatte. Der in den $1980 e r$ Jahren eine Zeitlang vorhandene vorsichtige Optimismus zugunsten eines weiteren Aufschwungs der wissenschaftlichen Weiterbildung hatte sich in den folgenden Jahren nicht bewahrheitet. Eher ließ sich eine Phase der Stagnation beobachten. In den 199oer Jahren dominierte daher eine Debatte über die Barrieren und Hindernisse, die der Weiterbildung an den Hoch- 
schulen im Wege standen (AUE 1990). Unterschiedliche Ursachen wurden dafür geltend gemacht: an erster Stelle die hohe Studiennachfrage und Auslastung in der akademischen Lehre, die ausgeprägte Forschungs- und Angebotsorientierung der Hochschulen, die mit der stärkeren Bedarfsorientierung von Weiterbildung nur teilweise kompatibel sei, haushalts- und personalrechtliche Hürden, die einem größeren Engagement der Hochschulen in der Weiterbildung im Wege stünden, und andere mehr. Seit Ende der 1990er Jahre kam dann die Frage auf, ob mit dem 1999 eingeleiteten Bologna-Prozess ein Wendepunkt für die Weiterbildung an Hochschulen verbunden sei.

Nachdem das Thema Bologna bereits auf den vorangegangenen Jahrestagungen der DGWF aufgegriffen worden war, hatte erstmalig die Wiener Jahrestagung 2005 die Bedeutung des Bologna-Prozesses für die wissenschaftliche Weiterbildung breit thematisiert (Cendon/Marth/Vogt 2006). Unterschiedliche Einschätzungen standen sich hier gegenüber: Auf der einen Seite wurde angenommen, dass die Idee gestufter Studiengänge der Verankerung der Weiterbildung in der Studiengangsarchitektur entgegenkommen könnte. Auf der anderen Seite umfasste der Bologna-Prozess zwar das lebenslange Lernen, aber nicht unbedingt die wissenschaftliche Weiterbildung in ihrem herkömmlichen Verständnis. Das Verständnis lebenslangen Lernens konzentrierte sich hier vielmehr primär auf solche Komponenten wie „recognition of prior learning“, Zugangswege für nicht-traditionelle Studierende oder flexible Lernpfade im Studium (Banscherus 2010), die weniger mit Weiterbildung nach einem ersten Studienabschluss als mit dem Erwerb dieses Abschlusses selbst verbunden sind. Daraus ergaben sich zwei Schlussfolgerungen: Es galt zum einen, die Impulse des Bologna-Prozesses, die sich aus der neuen Perspektive des lebenslangen Lernens ergaben, in der wissenschaftlichen Weiterbildung aufzugreifen und dabei insbesondere den Blick auf neue Zielgruppen zu richten. Zum anderen war aber davon auszugehen, dass das bisherige Feld der postgradualen Weiterbildung noch gar nicht im Blickfeld des Bologna-Prozesses lag.

Im Anschluss an die Wiener Jahrestagung entstand in einem Gespräch zwischen einem Vertreter des Bundesministeriums für Bildung und Forschung (BMBF) und Wissenschaftlern, die sich für die wissenschaftliche Weiterbildung engagierten, die zunächst noch sehr abstrakte Idee, mit einem Förderprogramm einen Anreiz für neue Initiativen und Maßnahmen im Bereich der Weiterbildung an Hochschulen zu schaffen. Ein solches Programm sollte beiden Zielen dienen: der Förderung der wissenschaftlichen Weiterbildung im bisherigen Sinne und der Erschließung neuer Zielgruppen durch innovative Angebote und Formate. Hierbei gab es jedoch drei Hindernisse zu überwinden: Erstens wurde zu diesem Zeitpunkt gerade im Zuge der Föderalismusreform die hochschulpolitische Kompetenzverteilung zwischen Bund und Ländern neu ausgehandelt, sodass ein solches Förderprogramm nicht in der Alleinregie des Bundes durchgeführt werden konnte, sondern zwischen Bund und Ländern abgestimmt werden musste. Dies zog sich hin, auch aufgrund der Mitfinanzierung des Wettbewerbs durch die EU. Zweitens gab es (und gibt es formell immer noch) auf Länderebene die unglückliche Vorgabe der Kultusministerkonfe- 
renz, nach der es nur den Typus eines weiterbildenden Masterstudiengangs gibt, aber weder weiterbildende Bachelorstudiengänge noch andere Programmtypen (z. B. Zertifikatsprogramme). Drittens waren damals die Regelungen für den Hochschulzugang beruflich Qualifizierter ohne schulische Studienberechtigung immer noch sehr restriktiv und weit von der angestrebten Durchlässigkeit zwischen beruflicher und akademischer Bildung entfernt.

Ein wesentlicher Zwischenschritt war dann die gemeinsame Vereinbarung zu einer Qualifizierungsinitiative für Deutschland zwischen Bund und Ländern auf dem Dresdner Bildungsgipfel im Oktober 2008 (Bundesregierung/Regierungschefs der Länder 2008). Diese wählt bereits den Topos „Aufstieg durch Bildung“ als Titel, der bereits auf eine lange und durchaus kontroverse Begriffsgeschichte zurückblicken kann (Banscherus/Wolter 2016), während der Begriff der „offenen Hochschule“ nur an einer Stelle und eher beiläufig auftaucht. In dieser Vereinbarung wurde bereits für das Jahr 2010 ein Bund-Länder-Wettbewerb „lebenslange wissenschaftliche Qualifizierung“ angekündigt, „durch den die Entwicklung von praxisnahen und ausbildungsbegleitenden Studiengängen an den Hochschulen gefördert und die Integration von Berufstätigen und beruflich Qualifizierten in die Hochschulbildung erhöht wird“. Damit war nicht nur die herkömmliche Engführung des Weiterbildungsbegriffs tendenziell aufgelöst, sondern auch die wesentliche Idee des Wettbewerbs formuliert worden. Der nächste Schritt war die von der Gemeinsamen Wissenschaftskonferenz (GWK) vorbereitete und beschlossene Bund-Länder-Vereinbarung über den Wettbewerb Aufstieg durch Bildung: offene Hochschulen vom Mai 2010, die Grundlage für die Ausschreibung des Wettbewerbs und die Bewilligung der ersten Projekte im Jahre 2011 war (GWK 2010; BMBF 2011).

Auf Länderebene fanden sich z.T. ähnliche Programme, allen voran das Modellprojekt "Offene Hochschule Niedersachsen“ (2009 bis 2012), an dem fünf niedersächsische Hochschulen beteiligt waren (Hanft/Brinkmann 2013). Niedersachsen ist ein Land, das schon seit Jahrzehnten eine Vorreiterrolle bei der Öffnung der Hochschulen für Berufstätige und der Zusammenarbeit von Erwachsenenbildung und Hochschulen gespielt hat. Zur Vorgeschichte und zu den begünstigenden Rahmenbedingungen des Wettbewerbs gehören unter anderem die ANKOM-Projekte zur Entwicklung und Erprobung von Verfahren der Anrechnung beruflicher Kompetenzen auf Hochschulstudiengänge (2006 ff.) und die Neuregelung des Hochschulzugangs für beruflich Qualifizierte ohne schulische Hochschulreife durch die Kultusministerkonferenz (2009). Von daher kann insgesamt gesagt werden, dass in diesem Zeitraum in Deutschland eine Art stille Revolution im Blick auf die Neubewertung beruflicher Qualifikationen für ein Hochschulstudium stattgefunden hat. 


\section{Programmkonturen}

Ausschreibung: In den Bekanntmachungen des Bundesministeriums für Bildung und Forschung (BMBF) zum Wettbewerb Aufstieg durch Bildung: offene Hochschulen für die erste Wettbewerbsrunde (vom März 2011) und die zweite Wettbewerbsrunde (vom Juli 2013) werden vier Ziele genannt. Dies sind (1) die dauerhafte Sicherung des Fachkräfteangebots, (2) die Verbesserung der Durchlässigkeit zwischen beruflicher und akademischer Bildung, (3) die schnellere Integration von neuem Wissen in die Praxis sowie (4) die Stärkung der internationalen Wettbewerbsfähigkeit durch eine nachhaltige Profilbildung der Hochschulen im Bereich des lebenslangen Lernens und beim berufsbegleitenden Studium. Diese Zielbestimmung bewegt sich weitgehend im Rahmen des aktuellen bildungs- und arbeitsmarktpolitischen Diskurses im postsekundären Bereich (vgl. Wolter/Banscherus 2015, S. 22 ff.), der diese Signalbegriffe - Fachkräftebedarf, Durchlässigkeit, Praxisbezug, Profilbildung, lebenslanges Lernen - in der einen oder anderen sprachlichen Variante alle kennt.

Zur Realisierung dieser Ziele sollen Projekte gefördert werden, welche die Entwicklung nachfrage- und bedarfsorientierter Studienangebote - Studiengänge, Module oder Zertifikatsprogramme - im Bereich berufsbegleitender und dualer Studiengänge sowie des lebenslangen Lernens an Hochschulen beabsichtigen. Dieses Spektrum ist deutlich weiter gezogen, als es dem traditionellen postgradualen Verständnis von Weiterbildung entspricht. Diese Angebote sollen die Hochschule für neue Zielgruppen öffnen. Sie richten sich primär an Personen, die bereits über eine berufliche Qualifikation (zum Beispiel den Abschluss einer dualen Berufsausbildung, einer Berufsfachschule bzw. einer beruflichen Fortbildung oder einen Hochschulabschluss) und über berufliche Erfahrungen aus einer Erwerbstätigkeit verfügen. Sie können über eine der herkömmlichen schulischen Berechtigungen für eine Studienaufnahme verfügen oder ein Studium über einen der Zugangswege für Berufstätige ohne herkömmliche schulische Studienberechtigung aufgenommen haben. Weitere Zielgruppen, die neben den Berufstätigen in der Ausschreibung des Wettbewerbs genannt werden, sind Personen mit Familienpflichten, Berufsrückkehrer/innen, Studienabbrecher/innen und arbeitslose Hochschulabsolvent/inn/en.

Auswahl und Verlauf: Der Wettbewerb gliedert sich in eine erste Wettbewerbsrunde mit einer Laufzeit von 2011 bis 2017 und eine zweite Wettbewerbsrunde mit einer Laufzeit von 2014 bis 2020.' Beide Runden unterteilen sich in jeweils zwei Förderphasen, von denen die erste eine mögliche Förderung der Projekte bis zu 3,5 Jahren und die zweite eine mögliche Förderung über einen Zeitraum von bis zu 2,5 Jahren vorsieht. Ziel der ersten Förderphase ist die Entwicklung und Erprobung von Maßnahmen zum lebenslangen Lernen an Hochschulen, einschließlich ihrer Fundierung durch Forschungsaktivitäten. Ziel der zweiten Phase ist die Vorbereitung der möglichst nachhaltigen Implementation und Realisierung dieser Maßnahmen. Für den Wettbewerb stehen von 2011 bis 2020 planmäßig 250 Millionen Euro zur Verfü-

1 Der folgende Überblick stützt sich auf Wolter/Banscherus 2015. 
gung, die aus Mitteln des Bundes und des Europäischen Sozialfonds (ESF) stammen.

Die Auswahl der Projekte der ersten Förderphase der ersten Wettbewerbsrunde erfolgte in einem zweistufigen Verfahren durch eine international besetzte Fachjury: Zunächst wurden 167 Antragsskizzen eingereicht, von denen 34 für die Ausarbeitung eines Vollantrags ausgewählt wurden. Aus den 34 ausführlichen Anträgen wählte die Jury 26 Vorhaben aus. Das BMBF bewilligte daraufhin im September 2011 für die erste Förderphase der ersten Wettbewerbsrunde diese 26 Projekte, davon 16 Einzel- und zehn Verbundprojekte. An den Projekten waren 23 Universitäten und gleichgestellte Hochschulen, 28 Fachhochschulen und drei Forschungseinrichtungen aus 14 Bundesländern beteiligt (Schultz 2012). Die Auswahl der Projekte der ersten Förderphase der zweiten Wettbewerbsrunde erfolgte ebenfalls durch eine Jury, allerdings in einem einstufigen Verfahren. Von den insgesamt 100 eingereichten Anträgen schlug die Jury 47 zur Förderung vor, sodass seit August 2014 weitere 47 Projekte, davon 37 Einzel- und zehn Verbundprojekte, gefördert werden. An den Projekten sind 20 Universitäten und gleichgestellte Hochschulen, 44 Fachhochschulen und eine Forschungseinrichtung aus allen 16 Bundesländern beteiligt (Hopbach 2014).

Angebote, Formate und Zielgruppen: Nach den Rückmeldungen aus den Projekten führen 27 Prozent der in der ersten Wettbewerbsrunde entwickelten Angebote zu einem Bachelorabschluss, 33 Prozent zu einem Mastergrad und 29 Prozent schließen mit einem Zertifikat ab. Während hier also Bachelor-, Master- und Zertifikatsangebote annähernd gleichgewichtig nebeneinanderstehen, dominieren in der zweiten Wettbewerbsrunde mit 45 Prozent die Zertifikatsprogramme, 24 Prozent sollen zu einem Bachelor- und 23 Prozent zu einem Masterabschluss führen. In beiden Wettbewerbsrunden stehen mit über 6o Prozent die berufsbegleitenden Formate deutlich im Vordergrund. Auch setzen die Projekte in beiden Runden mit 45 bzw. 54 Prozent in starkem Maße auf die Verbindung von Online- und Präsenzphasen („Blended Learning“) (Hartmann/Stamm/Ulbricht 2015; Hopbach 2014).

In beiden Runden entfällt mit 25 bzw. 32 Prozent der größte Anteil der geplanten Studienangebote auf die Fächergruppe der Ingenieurwissenschaften. Danach folgen mit 20 bzw. 23 Prozent die Rechts-, Wirtschafts- und Sozialwissenschaften. Auf die Fächergruppe Humanmedizin/Gesundheitswissenschaften entfallen 12 bzw. 20 Prozent der geplanten Angebote, 13 bzw. 14 Prozent auf die Fächergruppe Mathematik/ Naturwissenschaften. Die Fächergruppe Sprach- und Kulturwissenschaften kommt mit 2 bzw. 4 Prozent nur auf ausgesprochen geringe Anteilswerte. Ähnliches gilt für die weiteren Fächergruppen. Mit Blick auf die Zielgruppen der Projekte richten sich mit 21 bzw. 18 Prozent die geplanten Angebote in beiden Runden am häufigsten an Berufstätige. Danach folgen mit 20 bzw. 19 Prozent Personen mit Familienpflichten. Beruflich qualifizierte Studieninteressierte ohne schulische Studienberechtigung sind für 15 bzw. 11 Prozent der geplanten Angebote eine relevante Zielgruppe. Im Unterschied zur ersten Runde, in der sie noch kaum eine Rolle gespielt haben, sind in 
der zweiten Runde mit 13 Prozent der Nennungen aus den Projekten auch die Absolvent/inn/en von Bachelorstudiengängen eine relevante Zielgruppe (Schultz 2012; Hopbach 2014). Die anderen in der Ausschreibung genannten Zielgruppen spielen dagegen eine untergeordnete Rolle. Zwischen den Zielgruppen gibt es allerdings einige Überschneidungen, so etwa zwischen Berufstätigen und Personen mit Familienpflichten.

Infrastruktur: Bei der Entwicklung und Implementation der geplanten Studienformate werden die Projekte vom Projektträger, der VDI/VDE Innovation und Technik $\mathrm{GmbH}$ (VDI/VDE-IT), fachlich und administrativ betreut. Außerdem werden sie von der wissenschaftlichen Begleitung unterstützt, die im Zeitraum 2011 bis 2015 gemeinsam von Wissenschaftler/inne/n der Carl von Ossietzky Universität Oldenburg, der Deutschen Universität für Weiterbildung (DUW) in Berlin und der Humboldt-Universität zu Berlin (HU) durchgeführt wurde. Die wissenschaftliche Begleitung hat ihre Ergebnisse und Erfahrungen aus der ersten Phase der ersten Wettbewerbsrunde soeben in einer dreibändigen Veröffentlichung vorgelegt (Wolter/ Banscherus/Kamm 2016; Hanft/Brinkmann/Kretschmer/Maschwitz/Stöter 2016; Cendon/Mörth/Pellert 2016). Seit 2016 wird die wissenschaftliche Begleitung von der FernUniversität Hagen, der Universität Oldenburg, dem Centrum für Hochschulentwicklung (CHE) und der Technischen Universität Dortmund durchgeführt. Mit der Evaluation der einzelnen Projekte sowie des Wettbewerbs insgesamt wurde eine Arbeitsgruppe des Deutschen Zentrums für Hochschul- und Wissenschaftsforschung (DZHW) beauftragt.

\section{Offene Hochschule: Begriff und Konzept}

Während „Aufstieg durch Bildung“ als Titel der Dresdner Vereinbarung von Bund und Ländern (2008) eher die Ambition des gesamten Programms kennzeichnet, das sich ja auf nahezu alle Sektoren des Bildungssystems, nicht nur auf die Hochschule bezieht, beansprucht der Begriff Offene Hochschule ohne Zweifel einen engeren Bedeutungsraum. Aber beide Begriffe sind nicht eindeutig definiert. Während „Aufstieg durch Bildung" schon eine längere Begriffsgeschichte aufweist und eine eher gesellschaftspolitisch mehrdeutige und umstrittene, nicht ganz ideologiefreie Kategorie ist, ist der Begriff Offene Hochschule in Deutschland nicht sehr etabliert. In gewisser Weise ist es sogar mutig, diesen Begriff für ein hochschulpolitisches Programm zu reklamieren. Denn historische Entwicklung und akademisches Selbstverständnis der deutschen Universität haben noch nie ein Selbstbild oder eine Zielvorstellung als offene Hochschule nahegelegt. Im Gegenteil: Die deutsche Universität hat sich historisch immer eher als Gegenmodell einer offenen Hochschule verstanden.

Die exklusive Bindung des Hochschulzugangs an das Gymnasium, ein akademischer Ausbildungsauftrag, der sich historisch explizit in Distinktion zur beruflichen Bildung begriff, der Anspruch fast aller Hochschulen (auch jenseits der Exzellenz- 
initiative) als akademische Exzellenzeinrichtung (man lese die Leitbilder vieler Hochschulen, die auf das Prinzip der Bestenauslese setzen) - alles das widersprach und widerspricht weithin der Idee einer offenen Hochschule. So hatte auch die Kasseler LESSI-Studie unter den befragten Universitätsprofessoren und -professorinnen eine recht eindeutige Zurückhaltung bei der Bewertung solch hochschulpolitischer Ziele wie erweiterte Zugangsmöglichkeiten ohne Abitur, Anrechnung beruflicher Leistungen auf Studienanforderungen, Anhebung der Studienanfängerquote oder berufsbegleitende Studiengänge ergeben (Schomburg/Flöther/Wolf 2012, S.77) alles Kernvorstellungen des Wettbewerbs Offene Hochschule. Dass die deutsche Hochschule nicht nur programmatisch, sondern auch in ihren tatsächlichen Selektions- und Exklusionsmechanismen wenig offen ist, zum Beispiel im Blick auf „firstgeneration students“ oder „non-traditional students“, ist so vielfältig belegt (zuletzt Lange-Vester/Sander 2016, Merkel 2015), dass der Begriff der Offenen Hochschule zumindest in dieser weiten Semantik eher als euphemistisch zu gelten hat.

Innerhalb des Wettbewerbs lässt sich die Bedeutung des Begriffs Offene Hochschule mit mehreren konstitutiven Zielsetzungen und Handlungsansätzen umschreiben, die primär darum kreisen, die Hochschule in stärkerem Umfang für neue Zielgruppen zu öffnen, insbesondere solche, die Angebote lebenslangen Lernens in der Hochschule nachfragen. Dazu gehören, dem Wortlaut der Wettbewerbsdokumente (z. B. BMBF 2011) nach, mindestens folgende Komponenten: (1) Primäre Zielgruppe sind Berufstätige mit schulischer oder mit alternativer Zugangsberechtigung. (2) Es sollen neue Studienformate entwickelt werden wie berufsbegleitende oder duale Studienangebote oder Zertifikatsprogramme, die den besonderen Bedürfnissen von Berufstätigen entgegenkommen. (3) Es kann sich um Studienangebote in der akademischen Erstausbildung oder in der akademischen Weiterbildung handeln. (4) Diese sollen durch zielgruppenspezifische Maßnahmen (wie z. B. Anrechnung, medienbasiertes Lernen, Unterstützungsangebote) ergänzt werden. (5) Insgesamt sollen Strukturen Lebenslangen Lernens stärker an den Hochschulen verankert werden. Insbesondere soll die Durchlässigkeit zwischen beruflicher und akademischer Bildung vergrößert werden.

Durch die Zusammenschau dieser Komponenten lässt sich der Begriff „Offene Hochschule“ innerhalb des Wettbewerbs genauer umreißen, auch wenn er bildungspolitisch unterschiedlich auslegbar bleibt. In einer stärker systematischen Form können mehrere Definitionsansätze unterschieden werden, die nicht trennscharf sind, sondern eine breite Zone der Überschneidung aufweisen (vgl. Banscherus/Wolter 2016):

- Zugangsbasierter Ansatz: Offene Hochschule als offenere Form des Hochschulzugangs, insbesondere für Berufstätige ohne herkömmliche schulische Studienberechtigung („widening participation“);

- systemischer Ansatz: Offene Hochschule als Konzept zur Überwindung der institutionellen Segmentierung (bzw. zur Förderung der Durchlässigkeit) zwischen beruflicher und akademischer Bildung; 
- nachfrageorientierter Ansatz: Offene Hochschule als Konzept zur Erschließung neuer Zielgruppen für eine akademische Ausbildung mit dem Ziel, die Beteiligung an Hochschulbildung zu erhöhen (,increasing participation“);

- formatorientierter Ansatz: Offene Hochschule als Strategie zur Entwicklung und Implementation flexibler Studienstrukturen jenseits des herkömmlichen Vollzeit-Präsenzmodells;

- medienbasierter Ansatz: Offene Hochschule als technologiebasierter Ansatz zur Förderung selbstgesteuerten Lernens;

- weiterbildungsorientierter Ansatz: Offene Hochschule als Strategie zum Ausbau der Angebote im Bereich der wissenschaftlichen Weiterbildung und des Lebenslangen Lernens an Hochschulen.

Diese verschiedenen Ansätze stehen jeweils in ganz unterschiedlichen bildungspolitischen und bildungswissenschaftlichen Kontexten. Der zugangsorientierte und der systemische Ansatz entsprechen der Forderung nach einer Öffnung der Hochschulen für qualifizierte Berufstätige, die keine schulische Hochschulzugangsberechtigung erworben haben. Dies steht in der langen Traditionslinie der Forderung nach Gleichwertigkeit zwischen allgemeiner und beruflicher Bildung als Gegenmodell zur historisch etablierten Segmentierung zwischen beiden Bereichen - von Martin Baethge treffend als das deutsche „Bildungsschisma“ bezeichnet (Baethge 2007). Der nachfrageorientierte Ansatz greift eher auf bildungsökonomische, komparative und demografische Argumentationen zurück, wonach Deutschland im internationalen Vergleich eine für eine postindustrielle, wissensbasierte Ökonomie sehr niedrige Studienanfängerquote aufweist oder die Hochschulen neue Potenziale angesichts der zu erwartenden, demografisch bedingt rückläufigen Studiennachfrage erschließen müssen.

Der format- und der medienbasierte Ansatz stehen dagegen in der Tradition der Distance-Learning- und Open-University-Bewegung, die in Deutschland zunächst lange Zeit unter dem Stichwort „Studium neben dem Beruf“ stand. Dieser Ansatz liegt eher auf einer instrumentellen Ebene und stellt eher Wege und Maßnahmen zur Realisierung einer offenen Hochschule dar. Damit ist jedoch ein historischer oder bildungspolitischer Paradigmenwechsel verbunden. Für Jahrzehnte konzentrierte sich die bildungspolitische Debatte über eine Öffnung der Hochschule in erster Linie auf die Öffnung des Zugangs zur Hochschule. Erst spät wurde registriert, dass wesentliche Hürden für Berufstätige nicht allein in den Zugangswegen und Zulassungsverfahren bestehen, sondern in einer wenig inklusiven („conducive“), zielgruppenspezifischen Studienorganisation. Der weiterbildungsorientierte Ansatz knüpft schließlich an die Entwicklung von der volkstümlichen („extra-mural“) Hochschulbildung über die universitäre Erwachsenenbildung bis zur wissenschaftlichen Weiterbildung an (Wolter 2011).

Unter diesen verschiedenen Ansätzen haben der zugangs- und systemisch orientierte Ansatz ebenso wie der nachfrage- und der formatorientierte Ansatz für das Verständnis und die Begründung des Konzeptes „Offene Hochschule“ eine beson- 
dere Bedeutung gewonnen. In dieser Perspektive ist Offene Hochschule primär ein Konzept zur Erschließung neuer Zielgruppen für ein Hochschulstudium, unter denen die Berufstätigen, darunter wiederum diejenigen ohne schulische Studienberechtigung, ein besonderes Augenmerk finden, sowie zur Entwicklung und Erprobung zielgruppenadäquater Studienangebote und -formate. Damit rückt auch die Frage der Durchlässigkeit zwischen beruflicher und akademischer Bildung ins Zentrum.

\section{Wissenschaftliche Weiterbildung und Offene Hochschule}

Fragt man nach dem Verhältnis zwischen Offener Hochschule und wissenschaftlicher Weiterbildung, dann bleiben drei Schlussfolgerungen: Erstens sind beide Begriffe bzw. die dahinterstehenden Konzepte und Strategien nicht deckungsgleich, aber es gibt eine breite Zone der Überschneidung. Das Konzept Offene Hochschule ist deutlich weiter gefasst als die (traditionelle) wissenschaftliche Weiterbildung. Zweitens hat der Wettbewerb viele Anregungen und Impulse aufgenommen, die aus der jüngeren Entwicklungsgeschichte der wissenschaftlichen Weiterbildung hervorgegangen sind. Drittens hat der Wettbewerb die Weiterbildung an Hochschulen (im weiten wie im engen Sinne) in einem bislang unbekannten Umfang gefördert, aber es bleibt die Nachhaltigkeit als zentrale Herausforderung.

Die historische Entwicklung der Weiterbildung an den Hochschulen in Deutschland lässt sich in fünf Phasen differenzieren (für die Frühphasen Faulstich 1982, Schäfer 1988, Swoboda 1983; allgemein Wolter 2011, S.11 ff.). Am Ausgang des 19. Jahrhunderts setzt eine erste noch weitgehend vorinstitutionelle Initiative ein, unter solchen Bezeichnungen wie volkstümliche/s Hochschulkurse oder Vortragswesen. Sie knüpfte noch gar nicht an die professionellen Weiterbildungsbedürfnisse akademischer Berufsgruppen an, sondern an die von der englischen extra-mural-Bewegung verbreitete Idee einer öffentlichen Vermittlung wissenschaftlichen Wissens für ein außeruniversitäres Publikum. Diese Traditionslinie der Universitätsausdehnungsbewegung prägte auch noch die in den $1950 e r$ Jahren beginnende zweite Phase, die Institutionalisierung der universitären Erwachsenenbildung in (West-)Deutschland. Diese sah unter dem Namen Seminarkurse ihre Zielgruppe ebenfalls primär in einer bildungsbeflissenen Öffentlichkeit außerhalb der Universität und wollte eine Brücke zwischen Hochschulen und Erwachsenenbildung schlagen.

Die Mitte bzw. zweite Hälfte der 1970er Jahre: das ist die dritte Phase, die des Übergangs von der universitären Erwachsenenbildung zur wissenschaftlichen Weiterbildung. Dieser Begriffswechsel signalisiert einen Umbruch im Selbstverständnis. Stand bisher die Vermittlung wissenschaftlicher Expertise in die außeruniversitäre Erwachsenenbildung im Vordergrund, wurden jetzt die Hochschulabsolventinnen/-absolventen zu einer neuen, später zur zentralen Zielgruppe, und die Hochschule tritt weniger als Vermittlungsagentur zur Erwachsenenbildung, sondern als eigener Anbieter von Studiengängen, Weiterbildungsprogrammen oder Kursen auf. 
Das Spektrum variierte zwischen beruflicher Fortbildung von Erwerbstätigen mit Hochschulabschluss, Fortbildungsangeboten für Personen, die noch über keinen Studienabschluss verfügen, bis hin zu arbeitslosen Absolvent/inn/en. In diesen Zeitraum fallen auch die ersten größeren staatlichen Maßnahmen zur Förderung und Institutionalisierung der Weiterbildung an Hochschulen.

Die 1980er und frühen $1990 e r$ Jahre - dies ist dann, wie oben bereits erwähnt, eher eine Zeit der Stagnation als eines neuen Aufbruchs, in der die ausbleibende Einlösung der hochgesteckten Erwartungen, die Diskrepanz zwischen Programm und Realität der Weiterbildung an Hochschulen im Zentrum stand. Seit Ausgang der $1990 e r$ Jahre befindet sich die wissenschaftliche Weiterbildung in Deutschland stärker im Aufwind (Faulstich et al. 2007), und es setzt eine neue - die vierte - Phase ein. Inzwischen ist die Weiterbildung als dritte Säule (neben Lehre/Studium und Forschung) rechtlich fest im Aufgabenspektrum der Hochschulen verankert, wenn sich auch in der Praxis immer noch eine klare Hierarchie mit der Forschung an der Spitze und der Weiterbildung am Ende abzeichnet. Die Hochschulen haben nicht nur die Institutionalisierung der Weiterbildung vorangetrieben, sondern auch Umfang und Vielfalt ihrer Aktivitäten erweitert, begünstigt durch die Studienstrukturreform, die der Weiterbildung mit dem weiterbildenden Master einen eigenen Abschluss verschafft hat, wenn dieser bislang auch noch von geringer Bedeutung ist.

Allerdings ist diese Entwicklung mit einem tief greifenden Wandel im Selbstverständnis von Hochschulweiterbildung verbunden. Weiterbildung wird heute mehr im Kontext von Bildungsmanagement, Organisationsentwicklung und neuer Hochschulsteuerung (einschließlich Hochschulfinanzierung) gesehen - und weniger als Teil eines bildungstheoretisch und gesellschaftspolitisch legitimierten Bildungsauftrags der Universität. So hat Peter Faulstich die aus seiner Sicht drei zentralen Tendenzen in der wissenschaftlichen Weiterbildung, unverkennbar mit einem kritischen Unterton, als „Vermarktlichung“, „Managementisierung“ und „Bolognalisierung" bezeichnet (Faulstich 2010). Diese Phase geht fließend in eine fünfte über, für die das Paradigma des lebenslangen Lernens im europäischen Kontext - Bologna, Kopenhagen, Qualifikationsrahmen - zum Schlüsselbegriff geworden ist. Eine ihrer wichtigsten Manifestationsformen ist der Wettbewerb Offene Hochschule. Gegenüber dem traditionellen Verständnis wissenschaftlicher Weiterbildung, wie es bis in die 1990er Jahre dominierte, vollzieht der Wettbewerb einige wesentliche Erweiterungen, die durch den hochschulpolitischen und bildungswissenschaftlichen Weiterbildungsdiskurs in den letzten Jahren angestoßen wurden.

Neben postgradualen weiterbildenden Angeboten, also solchen nach einem ersten Studienabschluss, die aber meist für Teilnehmer/innen ohne ersten Abschluss offen waren, dann allerdings ohne Möglichkeit eines formellen Abschlusses, liegt jetzt ein Angebotsschwerpunkt im grundständigen Bereich, der früher nicht als weiterbildend galt. Die Grenzen zwischen Erststudium und weiterbildendem Studium werden fließend, indem Weiterbildung jetzt nicht mehr von der Studiengangsystematik, sondern von der vorangegangenen Bildungs- und Berufsbiografie definiert wird. 
Eine Folge davon ist, dass es nunmehr nicht nur weiterbildende Master-, sondern auch Bachelorstudiengänge gibt, die nach den KMK-Vorgaben zur Studiengangstruktur gar nicht vorgesehen sind, darüber hinaus Zertifikats- und Modulprogramme. Berufsbegleitende Angebote wurden bislang eher als ein Format postgradualer Studienangebote (oder des Fernstudiums, hier seit Langem auch schon im grundständigen Bereich) gesehen. Mit der Offenen Hochschule wird „berufsbegleitend“ jetzt auch zu einem Format grundständiger Studienangebote. Insgesamt führt die Offene Hochschule dazu, dass sich sowohl im Bereich grundständiger wie postgradualer Angebote eine deutliche Flexibilisierung abzeichnet, z. B. durch Teilzeitstudium, Hybridformate wie Blendend Learning oder online-basiertes Studium. Wie bereits erwähnt, wird auch das Spektrum an Zielgruppen erweitert. Damit gewinnt das Thema der Anrechnung beruflicher Qualifikationen auf Studienanforderungen eine hohe Relevanz (Lenz/Schmitt 2016; Hanft et al. 2014), ein Thema, das in der älteren wissenschaftlichen Weiterbildung keine Rolle spielte.

Aber diese Ansätze sind keine Neuerfindungen des Wettbewerbs Offene Hochschule, sondern fügen Anregungen, Empfehlungen oder Modelle zu einem in sich relativ konsistenten Programm zusammen, die von den Akteuren der Weiterbildung an Hochschulen schon vorher mit initiiert und befördert wurden. In jedem Fall hat sich mit dem Wettbewerb das Verständnis von Weiterbildung gegenüber den ersten drei Phasen, teilweise auch noch der vierten Phase in der Entwicklung wissenschaftlicher Weiterbildung im Sinne eines integrierten Konzepts lebenslangen Lernens erheblich erweitert. Begrifflich bieten sich jetzt drei Alternativen: (1) Der traditionelle Begriff wissenschaftlicher Weiterbildung löst sich von seiner postgradualen Engführung und umfasst zukünftig alle lebensverlaufsbezogenen Varianten von Weiterbildung an der Hochschule. (2) Der Begriff Offene Hochschule tritt an die Stelle der wissenschaftlichen Weiterbildung, diese bleibt aber ein Teil des neuen Konzepts. Ob er sich langfristig tatsächlich durchsetzt, ist allerdings offen, da - wie schon gesagt der Begriff Offene Hochschule nicht frei von ideologischen Konnotationen und Widerständen in der deutschen Hochschullandschaft ist. Von daher gibt es (3) noch die Möglichkeit, auf einen anderen passenderen Begriff zurückzugreifen. Dafür bietet sich zurzeit einzig der Begriff des lebenslangen (oder lebensbegleitenden) Lernens an.

Der Wettbewerb Aufstieg durch Bildung: offene Hochschulen ist schon vom Fördervolumen her das umfangreichste Förderprogramm zur Implementierung von Strukturen lebenslangen Lernens im deutschen Hochschulsystem, das bislang initiiert wurde. Die geförderten Projekte haben eine Art Pilotfunktion, sie dienen der innovativen Modellentwicklung für Studiengänge, Unterstützungsangebote, neue medienbasierte Lernformen, Anrechnungsverfahren und andere Handlungsansätze, die auch anderen Hochschulen als Anregung und Modell dienen können. Bund und Länder setzen mit diesem Wettbewerb ein deutliches Zeichen, dass es ihnen ernst ist mit der Öffnung der Hochschulen für Berufstätige und lebenslanges Lernen. Noch nie zuvor waren in ein solches Förderprogramm für die Weiterbildung so viele Institutionen des Wissenschaftsbereichs einbezogen. Die quantitativen Wirkungen 
dieser neuen Öffnungspolitik sind allerdings bislang eher ernüchternd. Nach wie vor fällt der Anteil der Hochschulen am gesamten Weiterbildungsmarkt in Deutschland mit deutlich weniger als fünf Prozent aller Teilnahmefälle sehr bescheiden aus (Kamm et al. 2016). Der Anteil nicht-traditioneller Studierender hat sich zwar in den letzten Jahren, unter anderem dank der „Meisterregelung“ (Anerkennung beruflicher Fortbildungsabschlüsse als allgemeine Hochschulreife), auf etwa drei Prozent aller Studienanfänger/innen vergrößert, liegt aber immer noch auf einem niedrigen Niveau (Otto/Kamm 2016; Wolter 2015).

Insgesamt stellt der Wettbewerb Aufstieg durch Bildung: offene Hochschulen eine Art Studienreformlaboratorium der Hochschulentwicklung in Deutschland dar, in dessen Rahmen neue Studienstrukturen entwickelt und erprobt werden, die sich unter anderem durch das Prinzip der Teilnehmerorientierung und der Flexibilisierung von dem bislang dominierenden Studientyp unterscheiden und möglicherweise eine Folie für die Studienreformen des nächsten Jahrzehnts bilden. Unter diesem Aspekt lässt sich vielleicht als Schlussfolgerung feststellen, dass der Wettbewerb insgesamt eher von qualitativer als von quantitativer Bedeutung ist. Die wichtigste Herausforderung für Programm und Projekte (und der wichtigste Prüfstein für den Erfolg) wird die Sicherung der Nachhaltigkeit sein, die Umsetzung von "Modellprojekten“ in Regelstrukturen an den beteiligten Hochschulen und im Hochschulsystem insgesamt.

\section{Literatur}

AUE, Arbeitskreis Universitäre Erwachsenenbildung (Hrsg.) (1990): Hemmnisse und Desiderata bei der Realisierung wissenschaftlicher Weiterbildung durch die Hochschulen. Hannover.

Baethge, M. (2007): Das deutsche Bildungs-Schisma: Welche Probleme ein vorindustrielles Bildungssystem in einer nachindustriellen Gesellschaft hat? In: Lemmermöhle, D./ Hasselhorn, M. (Hrsg.): Bildung - Lernen. Göttingen. S. 93-116.

Banscherus, U. (2010): Lebenslanges Lernen im Bologna-Prozess. In: Wolter, A./Wiesner, G./Koepernik, C. (Hrsg.): Der lernende Mensch in der Wissensgesellschaft. Perspektiven lebenslangen Lernens. Weinheim. S. 221-238.

Banscherus, U./Wolter, A. (2016): Aufstieg durch Bildung: offene Hochschulen - Annäherungen an ein Begriffspaar. In: Wolter, A./Banscherus, U./Kamm, C. (Hrsg.): Zielgruppen Lebenslangen Lernens an Hochschulen. Münster. S. 31-52.

BMBF, Bundesministerium für Bildung und Forschung (2011): Bekanntmachung von Richtlinien zum Wettbewerb „Aufstieg durch Bildung: offene Hochschulen“. Berlin.

Bundesregierung/Regierungschefs der Länder (2008): Aufstieg durch Bildung. Die Qualifizierungsinitiative für Deutschland. Dresden. 
Cendon, E./Marth, D./Vogt, H. (Hrsg.) (2006): Wissenschaftliche Weiterbildung im Hochschulraum Europa. Hamburg.

Cendon, E./Mörth, A./Pellert, A. (Hrsg.) (2016): Theorie und Praxis verzahnen. Lebenslanges Lernen an Hochschulen. Ergebnisse der wissenschaftlichen Begleitung des BundLänder-Wettbewerbs Aufstieg durch Bildung: offene Hochschulen. Band 3. Münster.

Faulstich, P. (1982): Erwachsenenbildung und Hochschule. Bestandsaufnahme, Modelle, Perspektiven. München.

Faulstich, P. (2010): Die deutsche Perspektive: Mittlere Systematisierung im Spannungsfeld zwischen Hochschule und Weiterbildung. In: Denk-doch-mal.de - Netzwerk Gesellschaftsethik e. V. www.denk-doch-mal.de/node/241.

Faulstich, P./Graeßner, G./Bade-Becker, U./Gorys, B. (2007): Länderstudie Deutschland. In: Hanft, A./Knust, M. (Hrsg.): Weiterbildung und lebenslanges Lernen an Hochschulen. Eine internationale Vergleichsstudie zu Strukturen, Organisation und Angebotsformen. Münster. S. 87-164.

GWK, Gemeinsame Wissenschaftskonferenz (2010): Bund-Länder-Vereinbarung über den Wettbewerb „Aufstieg durch Bildung: offene Hochschulen“ zur Förderung von Wissenschaft und Forschung an deutschen Hochschulen. Bonn.

Hanft, A./Brinkmann, K. (Hrsg.) (2013): Offene Hochschulen. Die Neuausrichtung der Hochschulen auf lebenslanges Lernen. Münster.

Hanft, A./Brinkmann, K./Gierke, W./Müskens, W. (2014): Anrechnung außerhochschulischer Kompetenzen in Hochschulstudiengängen. Oldenburg.

Hanft, A./Brinkmann, K./Kretschmer, S./Maschwitz, A./Stöter, J. (Hrsg.) (2016): Organisation und Management von Weiterbildung und Lebenslangem Lernen an Hochschulen. Ergebnisse der wissenschaftlichen Begleitung des Bund-Länder-Wettbewerbs Aufstieg durch Bildung: offene Hochschulen. Band 2. Münster.

Hartmann, E./Stamm, I./Ulbricht, L. (2015): Im Überblick: Der Bund-Länder-Wettbewerb Aufstieg durch Bildung: offene Hochschulen. In: Hanft, A./Pellert, A./Cendon, E./ Wolter, A. (Hrsg.): Weiterbildung und Lebenslanges Lernen an Hochschulen. Ergebnisse der wissenschaftlichen Begleitung zur ersten Förderphase der ersten Wettbewerbsrunde des Bund-Länder-Wettbewerbs: Aufstieg durch Bildung: offene Hochschulen. Oldenburg. S. 4-9.

Hopbach, A. (2014): Aufstieg durch Bildung: offene Hochschulen - Projekte der 2. Wettbewerbsrunde. In: Stamm, I./Tillack, D. (Red.): Auftakt zur 2. Wettbewerbsrunde des Bund-Länder-Wettbewerbs Aufstieg durch Bildung: offene Hochschulen. Berlin, 7. Oktober 2014 - Dokumentation. Bielefeld. S. 12-21.

Kamm, C./Schmitt, S./Banscherus, U./Wolter, A. (2016): Hochschulen auf dem Weiterbildungsmarkt: Marktposition und Teilnehmerstruktur. Ergebnisse einer sekundäranalytischen Untersuchung. In: Wolter, A./Banscherus, U./Kamm, C. (Hrsg.): Zielgruppen Lebenslangen Lernens an Hochschulen. Ergebnisse der wissenschaftlichen Begleitung des Bund-Länder-Wettbewerbs Aufstieg durch Bildung: offene Hochschulen. Band 1. Münster. S. $137-164$.

Lange-Vester, A./Sander, T. (Hrsg.) (2016): Soziale Ungleichheiten, Milieus und Habitus im Hochschulstudium. Weinheim. 
Lenz, K./Schmitt, S. (2016): Anrechnung von außerhochschulisch erworbenen Kompetenzen als Instrument zur Öffnung der Hochschulen. In: Wolter, A./Banscherus, U./ Kamm, C. (Hrsg.): Zielgruppen Lebenslangen Lernens an Hochschulen. Ergebnisse der wissenschaftlichen Begleitung des Bund-Länder-Wettbewerbs Aufstieg durch Bildung: offene Hochschulen. Band 1. Münster. S.321-344.

Merkel, M. C. (2015): Bildungsungleichheit am Übergang in die Hochschule. Weinheim.

Otto, A./Kamm, C. (2016): „Ich wollte einfach noch eine Stufe mehr“. Vorakademische Werdegänge und Studienentscheidungen von nicht-traditionellen Studierenden und ihr Übergang in die Hochschule. In: Wolter, A./Banscherus, U./Kamm, C. (Hrsg.): Zielgruppen Lebenslangen Lernens an Hochschulen. Ergebnisse der wissenschaftlichen Begleitung des Bund-Länder-Wettbewerbs Aufstieg durch Bildung: offene Hochschulen. Band 1. Münster. S.197-224.

Schäfer, E. (1988): Historische Vorläufer der wissenschaftlichen Weiterbildung. Von der Universitätsausdehnungsbewegung bis zu den Anfängen der universitären Erwachsenenbildung in der Bundesrepublik Deutschland. Opladen.

Schomburg, H./Flöther, C./Wolf, V. (2012): Wandel von Lehre und Studium an deutschen Hochschulen - Erfahrungen und Sichtweisen der Lehrenden (LESSI-Studie). Projektbericht INCHER-Kassel.

Schultz, E. (2012): Ergebnis der Projektauswahl für die erste Wettbewerbsrunde. In: Stamm-Riemer, I./Tillack, D. (Red.): Auftakt des Bund-Länder-Wettbewerbs „Aufstieg durch Bildung: offene Hochschulen“. Berlin, 9. Dezember 2011 - Dokumentation. Bonn/Berlin. S.13-19.

Swoboda, W. H. (1983): Universitäre Erwachsenenbildung. Die deutschen Hochschulen von der Populärbildung zum weiterbildenden Studium. Köln/Wien.

Wolter, A. (2011): Die Entwicklung wissenschaftlicher Weiterbildung in Deutschland: Von der postgradualen Weiterbildung zum lebenslangen Lernen. In: Beiträge zur Hochschulforschung, 33 (4), S. 8-35.

Wolter, A. (2015): Von der Öffnung des Hochschulzugangs zur offenen Hochschule: Der Hochschulzugang für Berufstätige im Wandel. In: Recht der Jugend und des Bildungswesens. S. 291-308.

Wolter, A./Banscherus, U. (2015): Der Bund-Länder-Wettbewerb Aufstieg durch Bildung: offene Hochschulen im Kontext der (inter-)nationalen Diskussion um lebenslanges Lernen. In: Klages, B./Bonillo, M./Reinders, S./Bohmeyer, A. (Hrsg.): Gestaltungsraum Hochschullehre. Potenziale nicht-traditioneller Studierender nutzen. Opladen. S. 17-35.

Wolter, A./Banscherus, U. (2016): Lebenslanges Lernen und akademische Bildung als internationales Reformprojekt. In: Wolter, A./Banscherus, U./Kamm, C. (Hrsg.): Zielgruppen Lebenslangen Lernens an Hochschulen. Münster. S. 53-80.

Wolter, A./Banscherus, U./Kamm, C. (Hrsg.) (2016): Zielgruppen Lebenslangen Lernens an Hochschulen. Ergebnisse der wissenschaftlichen Begleitung des Bund-Länder-Wettbewerbs Aufstieg durch Bildung: offene Hochschulen. Band 1. Münster. 


\section{Beruflichkeit und wissenschaftliche Weiterbildung}

BERND KAßEBAUM

\section{Einleitung}

Man darf sich nicht täuschen lassen: Obwohl nach der jüngsten Erhebung im Erwachsenenbildungssurvey in Deutschland im Jahr 2014 knapp 50 Prozent der befragten Erwerbstätigen an einer Maßnahme der betrieblichen Weiterbildung (und gut zwei Drittel an einer Weiterbildungsmaßnahme insgesamt) teilgenommen haben, sind doch strukturelle Defizite offenkundig. Weit mehr als die Hälfte der betrieblichen Weiterbildungen sind kürzer als ein Tag. Je kleiner die Betriebe und je prekärer die Beschäftigungssituation in den Branchen und Erwerbsformen, desto geringer ist die Weiterbildungsbeteiligung. Vor allem ist die Weiterbildungsbeteiligung extrem abhängig vom Bildungsstand und damit auch Resultat sozialer Benachbzw. Bevorteilung. Je höher das Einkommen bzw. je höher der berufliche Abschluss, desto höher ist die Beteiligung an Weiterbildung. Bei erwerbstätigen Akademikerinnen und Akademikern und bei Erwerbstätigen mit mittleren beruflichen Abschlüssen beträgt die Teilnahmequote 72 Prozent, bei Absolventinnen und Absolventen der dualen oder vollschulischen beruflichen Bildung 53 Prozent, bei formal gering qualifizierten Beschäftigten 44 Prozent. Die Beteiligungsquote von Erwerbslosen an Weiterbildung insgesamt liegt bei 32 Prozent und an der betrieblichen Weiterbildung im Rahmen von arbeitsmarktpolitischen Maßnahmen bei 11 Prozent. Zudem ist mit den arbeitsmarktpolitischen Entscheidungen der letzten Jahre massiv auf die Qualität von Weiterbildung im Rahmen der Arbeitsförderung eingewirkt worden. Weiterbildung - so der ehemalige Geschäftsführer des IAQ, Prof. Gerhard Bosch - ist „mit der Agenda 2010 aus der Mode gekommen“ (Bosch 2016).

Weiterbildung ist und bleibt in der Bildungspolitik der Bundesrepublik ein Stiefkind. Von einer vierten institutionalisierten Säule des Bildungssystems, wie in bildungsreformerischer Zielsetzung schon im Bildungsgesamtplan 1973 von der damaligen Bund-Länder-Kommission angedacht, ist Weiterbildung heute trotz der Bildungsurlaubs- oder Bildungsfreistellungsgesetze in den meisten Bundesländern noch immer weit entfernt. Politik und Wirtschaft sind nicht gewillt, Weiterbildung 
umfassend gesetzlich zu regeln. Trotz einer Reihe von Weiterbildungstarifverträgen haben es auch die Sozialpartner bisher nicht vermocht, dem Thema eine größere Bedeutung zu geben und insbesondere nachhaltige Wege für diejenigen aufzuzeigen, die in den Maßnahmen unterrepräsentiert sind.

In diesen - hier nur angedeuteten - institutionellen und bildungspolitischen Kontext ist die Diskussion über die wissenschaftliche Weiterbildung einzubetten. Wissenschaftliche Weiterbildung spielt dabei im gesamten Weiterbildungsmarkt nach wie vor nur eine untergeordnete Rolle. Nach der Statistik des Berichtssystems Weiterbildung entfielen auf den Bereich der beruflichen Weiterbildung 2007 zwei Prozent der Teilnahmefälle, auf den Bereich der allgemeinen Bildung ca. fünf Prozent. Selbst bei den HochschulabsolventInnen sind die Angaben mit drei Prozent für die berufliche und sieben Prozent für die allgemeine Bildung nicht wesentlich höher. Die jüngeren Zahlen nach dem Adult Education Survey (AES) bestätigen diesen Sachverhalt. Hiernach deckt die Hochschule insgesamt drei Prozent der Nachfrage nach beruflicher Weiterbildung und speziell bei HochschulabsolventInnen 5,5 Prozent der Nachfrage an beruflicher Weiterbildung ab.

Auf den ersten Blick scheint die Teilnehmerzusammensetzung an Maßnahmen der wissenschaftlichen Weiterbildung heterogener $\mathrm{zu}$ sein als zu erwarten: So hat eine große Mehrzahl der Teilnehmenden eine Hochschulzugangsberechtigung, aber nur etwas mehr als 40 Prozent einen Hochschulabschluss, 30 Prozent dagegen einen dualen Aus- oder Fortbildungsabschluss (Zahlenangaben vgl. Wolter 2011; Kamm et al. 2016). Andrä Wolter führt dies u. a. darauf zurück, dass die wissenschaftliche Weiterbildung vielfach auch für die hochschulinternen Weiterbildungsmaßnahmen für die Beschäftigten verantwortlich ist und demnach das Berichtssystem Weiterbildung auch die hochschulinterne Weiterbildung als Bestandteil der wissenschaftlichen Weiterbildung erfasst hat. Jedoch bleibt auch in dieser Statistik festzuhalten, dass nur die wenigsten Teilnehmerinnen und Teilnehmer an Maßnahmen der wissenschaftlichen Weiterbildung einen manuellen Beruf erlernt haben (Faulstich/ Oswald 2012). In der Mehrheit der Angebote wird ein Hochschulabschluss vorausgesetzt. Bei weiterbildenden Studiengängen liegt dieser Anteil bei mehr als 8o Prozent. „Nur in Ausnahmefällen kann die Zugangsberechtigung durch die Verbindung von Hochschulreife, Berufsausbildung und Berufserfahrung erlangt werden" (ebd., S. 381). In nur 1,4 Prozent der Fälle wird eine abgeschlossene Berufsausbildung als Zugangsvoraussetzung akzeptiert (ebd.).

Angesichts dieser Rahmendaten ist eine Reform der Weiterbildungspolitik in Deutschland mehr als dringend geboten. Weiterbildung ist angesichts des demografischen Wandels, angesichts der zunehmenden Digitalisierung der Arbeitswelt und angesichts der erforderlichen Integration von formal gering Qualifizierten oder Erwerbslosen in den Arbeitsmarkt ein wesentlicher Schlüssel zur Sicherung von Beschäftigung und zur Deckung des Fachkräftebedarfs. Die gewerkschaftlichen Reformvorschläge zur Weiterbildung liegen zum Teil seit Jahren vor. Dazu gehören elementar die Regelung der Lernzeiten, der finanziellen und organisatorischen Rah- 
menbedingungen für die Lernenden, die finanzielle Unterstützung der Lernenden, die Qualitätssicherung, die Sicherung der politischen und kulturellen Weiterbildung im Angebotsspektrum und insbesondere in der beruflichen und betrieblichen Weiterbildung die berufliche Verwertbarkeit der Maßnahmen. Nicht zu vergessen ist der Aufbau von Informations- und Beratungsstrukturen; sie sind insbesondere für die Beschäftigten besonders notwendig, die bisher an Weiterbildungsmaßnahmen unterdurchschnittlich teilnehmen, und sollten möglichst am Arbeitsplatz beginnen. $\mathrm{Ob}$ es dabei um die stärkere Ausrichtung an abschlussbezogenen Weiterbildungsmaßnahmen oder um die Anerkennung informell erworbener Kompetenzen geht, wünschenswert ist aus Sicht der Beschäftigten eine Stärkung der beruflichen Handlungskompetenz, um damit ihre Beschäftigungsverhältnisse zu sichern, Effekte für bessere Arbeit und Aufstiegsmöglichkeiten zu erzielen und um ihr Entgelt abzusichern oder zu verbessern.

\section{Annäherungen an ein Verständnis wissenschaftlicher Weiterbildung}

Auf der Homepage des BMBF lauten die ersten Zeilen zur wissenschaftlichen Weiterbildung: „Wissenschaftliche Weiterbildung im Rahmen von lebenslangem Lernen dient der Entwicklung des eigenen Potenzials, der Sicherung des Arbeitsplatzes und der beruflichen Karriere und festigt den Wirtschaftsstandort Deutschland." Und weiter: „Wissenschaftliche Weiterbildung wird von Hochschulen und anderen Bildungseinrichtungen in einer Vielzahl von Formaten angeboten (...) Die Teilnahme steht nicht nur denen offen, die bereits ein Hochschulstudium abgeschlossen haben, da die erforderlichen Voraussetzungen auch auf andere Weise nachgewiesen werden können“ (BMBF 2016).

An dieses Zitat lassen sich eine Reihe von Fragen anschließen, die sich sowohl auf das Selbstverständnis wie auf die Verwirklichung von hier formulierten Ansprüchen beziehen lassen. Historisch aus der universitären Erwachsenenbildung entstanden, bildet wissenschaftliche Weiterbildung heute ein in einer Vielzahl von Formaten und zumeist über Zentraleinrichtungen der Hochschulen organisiertes und nach Maßgabe der Landeshochschulgesetze zur kostendeckenden Arbeit verpflichtetes hochschulisches Bildungsangebot ab. $\mathrm{Zu}$ den Angeboten gehören u.a. weiterbildende Studiengänge, Kurse und Seminare. Die DGWF schlägt in ihren Empfehlungen folgende Systematik vor:

1. Weiterbildende Masterstudiengänge

2. Weiterbildende bzw. berufsbegleitende Bachelorstudiengänge

3. Weiterbildende Zertifikatsstudien

4. Weiterbildungsmodule

5. Weiterbildungsseminare

6. Sonstige Weiterbildungsformate (vgl. DGWF 2010). 
Diese können berufsbegleitend und/oder als Teilzeitangebot angelegt und als Präsenz- oder Online-Angebot strukturiert sein.

Noch heute dominiert an vielen Hochschulen ein Selbstverständnis von wissenschaftlicher Weiterbildung als „postgradualer“ Weiterbildung, d.h. die Angebote setzen häufig einen Studienabschluss voraus. Dieses - tradierte - Verständnis von wissenschaftlicher Weiterbildung auf der Basis grundständiger Studienabschlüsse lässt sich relativ eindeutig definieren. „Danach ging bzw. geht es bei der wissenschaftlichen Weiterbildung primär um Weiterbildungsangebote für Erwerbstätige, die bereits über einen Studienabschluss verfügen“ (Wolter 2011, S.11). Damit ließen sich die Adressaten der Angebote, nämlich Absolventinnen und Absolventen grundständiger Studienprogramme, die Anbieterinstitution, nämlich die Hochschule oder eine ihr zugeordnete Einrichtung, und das Anspruchsniveau, nämlich die Orientierung an Wissenschaftlichkeit, eindeutig bestimmen.

Doch tatsächlich wird dieses Selbstverständnis von den Akteuren der wissenschaftlichen Weiterbildung, von Politik und Gesellschaft hinterfragt und ist durch eine Reihe von Rahmenbedingungen und Prozessen in der wissenschaftlichen Weiterbildung selbst in Fluss geraten. Zu nennen sind politische, gesellschaftliche und ökonomische Faktoren. Peter Faulstich führt sie auf drei Tendenzen der Hochschulentwicklung zurück, die „Vermarktlichung“, die „Managementisierung“ und die „Bolognalisierung“ (vgl. Faulstich 2010, zitiert nach Wolter 2011).

Ausgehend von einem breiten Diskurs über den demografisch und technologisch induzierten Bedarf an Fachkräften haben Europäische Kommission, Bundesregierung und Länder im Kontext von Konzepten des Lebenslangen Lernens verschiedene Programminitiativen und gesetzliche Regelungen herbeigeführt, die auch Einfluss auf die Ausgestaltung wissenschaftlicher Weiterbildung haben. Dies sind beispielhaft die über die Empfehlung der Kultusminister von 2009 herbeigeführten Änderungen in Landeshochschulgesetzen und Verordnungen zur Verbesserung des Hochschulzugangs für Berufserfahrene. $\mathrm{Zu}$ nennen ist die sog. ANKOM-Initiative, mit der die Anrechnung beruflich erworbener Kompetenzen im Studium und damit die Durchlässigkeit zwischen beruflicher und hochschulischer Bildung verbessert werden sollte (vgl. Freitag et al. 2015). Und letztendlich sind die im Bundesprogramm „Aufstieg durch Bildung: offene Hochschulen“ zusammengeführten Projekte zu erwähnen (vgl. die Teilstudien der wissenschaftlichen Begleitung wie Wolter et al. 2016 und Hanft et al. 2016).

Dabei ist offenkundig ein Widerspruch bis heute nicht ausgeräumt: Auch wenn sich mittlerweile bestätigt hat, dass allein die Bereitstellung formaler Wege nicht reicht, sondern auch die materiellen Rahmenbedingungen $\mathrm{zu}$ gestalten, Übergänge $\mathrm{zu}$ schaffen, qualifizierte Informations- und Beratungsstrukturen zu entwickeln, einfache Anrechnungsverfahren einzuführen und zu guter Letzt auch curriculare Änderungen herbeizuführen sind, scheint einerseits die Sensibilität des Hochschulsystems insgesamt und auch die der wissenschaftlichen Weiterbildung für die stärkere Integration Berufserfahrener schrittweise zuzunehmen. Das ist die gute Nachricht. 
Und zugleich ist festzuhalten, dass der Anteil von Berufserfahrenen unter den StudienanfängerInnen seit einem Höchststand Mitte der 199oer Jahre von 38 Prozent schrittweise bis 2012 auf einen Anteil von 22 Prozent gesunken ist. Der Anteil der Berufserfahrenen ohne Hochschulzugangsberechtigung ist dabei je nach Sichtweise in den letzten Jahren immerhin auf ca. drei Prozent gestiegen oder über diese Marke nicht hinausgebracht worden. Festzuhalten bleibt, dass Berufserfahrene mit und ohne Hochschulzugangsberechtigung in der Minderheit sind.

Wissenschaftliche Weiterbildung ist innerhalb des Hochschulsystems auf besondere Weise mit der „Vermarktlichung“ der Hochschulen und der Privatisierung der Hochschulangebote konfrontiert. Wissenschaftliche Weiterbildung wird zwar in den Hochschulgesetzen neben Lehre und Forschung als drittes Aufgabenfeld anerkannt, es muss sich aber in der Regel über Gebühren selbst finanzieren. Neue Management- und Steuerungsmethoden in Hochschulen, basierend auf einem internen „simulierten (Forschungs- und Bildungs-)Markt“ (Pasternack/von Wissel 2011, S.48), und von der Politik etablierter Wettbewerb zwischen den Hochschulen einschließlich der politisch gesteuerten Zuwächse im Bereich der privaten Hochschulen führen zu einem Ökonomisierungsdruck, dem sich auch wissenschaftliche Weiterbildung nicht entziehen kann. Wissenschaftliche Weiterbildung hat sich zudem dem Wettbewerb mit privaten Weiterbildungsanbietern zu stellen. Damit gerät wissenschaftliche Weiterbildung von zwei Seiten unter Druck: Einerseits unterliegt sie vollkommen unstrittig den Qualitätsansprüchen des Hochschul- und Wissenschaftssystems; andererseits muss sie nach den Regeln eines weitgehend privaten Weiterbildungsmarktes agieren. Dies stärkt Tendenzen zu einer immer stärkeren Ausdifferenzierung von Formaten, Zielgruppen und Themen, stärkt aber nicht zwingend die qualitativen Ansprüche an Inhalte und Formate.

Aus Sicht der Lernenden ergibt sich ein anderes Verständnis von Weiterbildung. Da kann bereits das Bachelorstudium nach beruflicher Erstausbildung und/oder einigen Jahren der Erwerbstätigkeit eine Weiterbildung sein. Oder es kann die Unterbrechung des Studiums durch Erwerbstätigkeit sein, die eine spätere Fortsetzung in einem Masterstudium im Sinne einer Weiterbildungsmaßnahme findet. Daraus lässt sich ableiten, dass ein allein institutionelles Verständnis wissenschaftlicher Weiterbildung nicht ausreichend ist. Je differenzierter und vielfältiger Lern- und Erwerbsbiografien werden, desto mehr stellt sich die Frage der Integration von wissenschaftlicher Weiterbildung in das sog. Regelangebot der Hochschule und überdies die der Gestaltung neuer Lernwege zwischen beruflicher und hochschulischer Bildung und damit die Verbesserung sozialer und beruflicher Durchlässigkeit. Für Andrä Wolter ist von Bedeutung, dass „sich das Verständnis von Weiterbildung immer mehr von der alten studiengangbasierten Definition entfernt und sich mehr und mehr zu einer biographie- und teilnehmerbezogenen Auslegung entwickelt“ (Wolter 2016).

Um noch ein Argument aus Sicht der Erwerbstätigen anzufügen: Im Regelfall werden Erwerbstätige im Rahmen betrieblicher Weiterbildung daran interessiert sein, dass sie ihre berufliche Handlungsfähigkeit zumindest in Bezug auf kurzfristige 
technische und/oder organisatorische Prozesse erhalten können. Im Rahmen beruflicher Weiterbildung werden Erwerbstätige an einer längerfristigen Erhaltung und Weiterentwicklung beruflicher Handlungskompetenz interessiert sein. Es scheint klärungsnotwendig, ob wissenschaftliche Weiterbildung sich als Teil beruflicher Weiterbildung verstehen will. Hierbei kann man sich gut vorstellen, dass die wissenschaftliche Weiterbildung gute Bedingungen beruflicher Weiterbildung für akademisch ausgebildete Beschäftigte finden sollte. Schwieriger wird es, wenn man von den Weiterbildungsinteressen von Facharbeitern, Technikern oder Meistern her denkt. Hier sind andere kulturelle und institutionelle „Übersetzungsarbeiten“ erforderlich und didaktische Schritte zu gehen, die auf gegenseitige Lernprozesse setzen.

Ganz anders stellt sich die Frage der Annäherung zwischen Institution und Subjekt(en) oder zwischen Lehrenden und Lernenden, wenn man den Vermarktlichungsprozess in der Arbeit zum Gegenstand macht. Obwohl die Gewerkschaften in den letzten Jahren durchaus Erfolge darin hatten, Leiharbeit einzuschränken, einen Mindestlohn einzuführen und weitere sozial- und tarifpolitische Standards zu setzen, ist die Prekarisierung als Thema längst nicht vom Tisch. Über arbeitsorganisatorische Konzepte wie die des Arbeitskraft-Unternehmers in den Betrieben oder die des Kontraktarbeiters als wieder neu verbreitete Form von Schein-Selbstständigkeit dringt die Vermarktlichung weiter in die Erwerbsarbeit ein.

Die Frage an dieser Stelle ist, wie Bildungsinstitutionen im Allgemeinen und wissenschaftliche Weiterbildung im Besonderen diese Prozesse thematisieren. Gibt es so etwas wie Arbeitswelt- und Berufsorientierung analog zum schulischen Bildungsangebot? Gibt es dafür gute Konzepte? Hat wissenschaftliche Weiterbildung - selbst Gegenstand der Ökonomisierung - die Kraft, den Erwartungen von großen Teilen der Wirtschaft an schnell verwertbaren Qualifikationen zu widerstehen? Kann wissenschaftliche Weiterbildung den hohen Anspruch an politische Bildung als Teil ihres Angebotsspektrums beibehalten? In welchem Verhältnis stehen die Vermarktlichungsprozesse in Erwerbsarbeit und der Druck zur Kommerzialisierung zu einer Wissenschaft, die sich auch kritisch mit diesen Rahmenbedingungen auseinandersetzen will?

Diese grundsätzlichen Fragen werden im Folgenden möglicherweise unbeantwortet bleiben. Indem aber der Versuch unternommen wird, den Diskurs über die Weiterentwicklung wissenschaftlicher Weiterbildung mit einem anderen Prozess zusammenzudenken, der in der Berufsbildungs- und Hochschulpolitik der IG Metall eine gewisse Bedeutung hat, sollen Hinweise in zwei Richtungen möglich sein. Die Hinweise könnten zu folgenden Thesen führen:

„Beruflichkeit“ als Bildungskonzept ist aufgrund seiner Bezüge zur Persönlichkeitsentwicklung und zur Gestaltungskompetenz ein geeigneter Ansatz, um dem Ansturm an kurzfristig auf dem Arbeitsmarkt zu verwertenden Qualifikationen zu begegnen. Es geht dabei um die Frage zukunftsgerechter beruflicher Qualifizierung auch mittels der Möglichkeiten wissenschaftlicher Weiterbildung in einem Wechselspiel zwischen Wissenschaftlichkeit und Berufsorientierung, aber auch um den Er- 
halt oder die Wiedererlangung des Politischen in der Beruflichen Bildung. Im Kern des Bildungskonzepts steht der Erwerb beruflicher reflexiver Handlungskompetenzen im Rahmen wissenschaftlicher Ausbildung.

„Beruflichkeit“ als übergreifendes Politikkonzept stellt die Frage nach dem Zu-, Mitoder Gegeneinander von allgemeiner und beruflicher Bildung, stellt also auch die Frage nach einer künftigen Architektur der Bildungssysteme und der Gestaltung der Übergänge sowie der sozialen und beruflichen Durchlässigkeit zwischen ihnen. Wissenschaftliche Weiterbildung ist von dieser Frage betroffen, weil sie sich einerseits als Angebotsstruktur innerhalb der Hochschulen neben Forschung und Lehre etablieren muss; andererseits wird sie auf neue Lernwege zwischen beruflicher Bildung und Hochschule, auf die Anforderungen nach sozialer und beruflicher Durchlässigkeit und auf die Prozesse in der beruflichen Weiterbildung insgesamt eingehen und sich somit in einer Gesamtarchitektur von Bildung verorten müssen.

\section{Das Leitbild der „erweiterten modernen Beruflichkeit“ der IG Metall}

In einem mehrjährigen und wissenschaftlich begleiteten Prozess hat die IG Metall ein Leitbild der „erweiterten modernen Beruflichkeit“ entwickelt und 2014 zur Diskussion gestellt (vgl. IG Metall 2014; Kuda et al. 2012; auch www.wap.igmetall.de). Der Bildungsausschuss beim Vorstand der IG Metall hat das Leitbild als Orientierung für die Berufsbildungspolitik verabschiedet. Die zeitgleich veröffentlichten „BerufsbildungsPerspektiven 2014" des wissenschaftlichen Beraterkreises von ver.di und IG Metall unterstützen die im Leitbild vorgeschlagene Neugestaltung des Verhältnisses von beruflicher und hochschulischer Bildung (Verdi/IG Metall 2014).

Ausgangspunkt der Überlegungen zu der Arbeit an dem Leitbild waren beschäftigungs-, arbeitsmarkt-, bildungs- und gesellschaftspolitische Herausforderungen, die zu der Überlegung führten, wie Beruflichkeit als Prinzip der Qualifizierung gestärkt und weiterentwickelt werden kann.

Die im Leitbild bearbeiteten Herausforderungen sind:

- die durch Deregulierung, Prekarisierung und Taylorisierung bezeichneten Tendenzen in der Erwerbsarbeit,

- die mit „Akademisierung der Arbeitswelt“ verbundenen bildungs- und arbeitspolitischen Prozesse sowie

- die Gefährdung des Berufskonzepts durch europäische Bildungspolitik.

In Beantwortung dieser Herausforderungen erweitert die IG Metall ihr Verständnis von Beruflichkeit über den Bereich der beruflichen Aus- und Fortbildung auf den Hochschulbereich. Dies ist der Kern des Leitbildes. Hierbei ist der Begriff der Erweiterung nicht nur räumlich zu verstehen, sondern auch inhaltlich als Prinzip der auf gegenseitigen Lernprozessen basierenden Weiterentwicklung von Beruflichkeit. Aktuell hat die IG Metall auf dem Leitbild aufbauend ein Thesenpapier „Digitalisierung 
der Arbeitswelt - Herausforderungen für die Berufsbildung“ veröffentlicht (IG Metall 2016). Sie nimmt Beruflichkeit damit auch als Gestaltungsprinzip digitaler Arbeitswelten auf.

Im Unterschied zum Begriff des Berufs, der als spezifisch gefasstes und im Konsens von Sozialpartnern und Bildungsinstitutionen entwickeltes Bündel von Arbeitsfähigkeiten und Kompetenzen verstanden und in seiner jeweiligen institutionellen Einbettung als Beruf nach BBiG, als schulischer oder als akademischer Beruf begriffen wird, zielt der Begriff der Beruflichkeit auf übergreifende, auf Bildungsprozesse und Arbeitsgestaltung bezogene Prinzipien und Qualitätsmaßstäbe. Beispielsweise wurde mit dem Konzept der „modernen Beruflichkeit“ im Rahmen der Berufsbildungsdebatte um die Reform der dualen Ausbildung ein Schritt weg von dem traditionellen, auf spezielle Berufe bezogenen Verständnis von Beruflichkeit hin zu einem auf ein Berufsfeld orientiertes, an den Arbeits- und Geschäftsprozessen ausgerichtetes und auf dem Konzept der umfassenden und reflexiven beruflichen Handlungskompetenz beruhendes Verständnis abgelöst. Als „erweiterte moderne Beruflichkeit“ stellt das Leitbild gemeinsame Prinzipien und Maßstäbe sowohl für die betrieblich-duale wie für die hochschulische (Berufs-) Bildung zur Diskussion.

„Beruflichkeit“ meint demnach im Verständnis des Leitbildes:

- definierte Qualitätsmaßstäbe für berufliches Lernen in Ausbildung und Studium,

- die Beteiligung der Sozialpartner bei der curricularen Entwicklung von Berufsbildern und Studiengängen entlang dieser Qualitätsansprüche und

- die Stärkung und Weiterentwicklung umfassender reflexiver beruflicher Handlungskompetenzen.

Im Leitbild der IG Metall zielt Beruflichkeit sowohl auf eine bestimmte Qualität von Lernprozessen wie von Erwerbsarbeit. Das Leitbild ist so untrennbar mit der gewerkschaftlichen Diskussion um „gute Arbeit“ verbunden. Im Diskurs über die Schlussfolgerungen der Digitalisierung der Arbeitswelt gibt Beruflichkeit eine Orientierung für die Gestaltung von Lern- und Arbeitsprozessen.

\subsection{Beruflichkeit als Bildungskonzept}

In seinem Verständnis als Bildungskonzept baut das Leitbild auf dem genannten Konzept der modernen Beruflichkeit auf. Neue Akzente traten hinzu. Dazu gehört z. B. die Frage, wie Beschäftigte angesichts weitreichender Prekarisierung und angesichts des Vordringens von Werkvertragsverhältnissen gestärkt werden können, konkret, wie in Ausbildung und Studium auch berufsbiografische und arbeitsgestaltende Kompetenzen ausgebildet werden können. Die zunehmende Digitalisierung wirft die Frage nach dem Verhältnis von Wissenschafts- und Erfahrungsorientierung sowohl in der beruflichen wie in der hochschulischen Qualifizierung auf, dort gemeinhin als „Verwissenschaftlichung“, hier als „Verberuflichung“ tituliert. Beruflichkeit als Bildungskonzept setzt an den Bedürfnissen und Interessen der Lernenden an. Es 
ist ein Gegenkonzept zu dem ausschließlich an den Anforderungen der Arbeitsmärkte ausgerichteten Konzept der „Employability“.

In dem Leitbild der IG Metall werden folgende 15 Aussagen zur Qualität beruflichen Lernens formuliert. Die Überschriften zu diesen Aussagen sind:

- Berufliches Lernen erfordert eine fachlich breite Qualifikation.

- Berufliches Lernen vermittelt Wissen, Handlungsfähigkeit und ermöglicht praktische Erfahrungen.

- Berufliches Lernen orientiert sich an Arbeits- und Geschäftsprozessen.

- Berufliches Lernen geschieht durch die Bewältigung von (berufstypischen) Aufgaben.

- Berufliches Lernen ist entdeckendes und forschendes Lernen.

- Berufliches Lernen ist Bildung.

- Berufliches Lernen ist soziales Lernen.

- Berufliches Lernen zielt auf die Reflexion und Gestaltung von Arbeit.

- Berufliches Lernen umfasst die Reflexion und Gestaltung von Lern- und Berufswegen.

- Berufliches Lernen bereitet auf die Berufsrolle vor.

- Berufliches Lernen fördert und entwickelt Identität.

- Berufliches Lernen verknüpft Erfahrungs- und Wissenschaftsorientierung.

- Berufliches Lernen zielt auf ein anderes Theorie-Praxis-Verhältnis.

- Berufliches Lernen hat unterschiedliche Zielorte.

- Berufliches Lernen schließt niemanden aus.

Die Auffassung, dass aufgrund der Digitalisierung der Arbeitswelt in großem Ausmaß ganze Berufe oder Berufsgruppen verschwinden (Frey/Osborne 2011), ist glaubhaft methodisch problematisiert und widerlegt worden (Pfeiffer 2015). Aus dem IAB ist eine Studie vorgelegt worden, die nicht nach der Verdrängung von Berufen, sondern nach den Substituierungspotenzialen in den Berufen auf der Basis von Tätigkeitsanalysen des gegenwärtigen Niveaus von Computerisierung fragt. Ihre Analysen zeigen einerseits, dass es in „nahezu allen Berufen Tätigkeiten gibt, die derzeit (noch) nicht von Computern übernommen werden können“ (Dengler/Matthes 2015). Gleichwohl kommt diese Studie zu dem Ergebnis, dass 15 Prozent der derzeit sozialversicherungspflichtig Beschäftigten in Berufen arbeiten, die einem Substituierungspotenzial von 70 und mehr Prozent ausgesetzt sind. Gegen diesen und vergleichbare Ansätze wendet Sabine Pfeiffer ein: „Mit zunehmender Automatisierung und Digitalisierung steigt zwangsläufig die Systemkomplexität und der (noch) nicht automatisierte oder algorithmisierte Umgang mit Störungen erfordert gerade deshalb umso mehr und nicht - wie so oft angenommen - weniger lebendiges Arbeitsvermögen zu ihrer situativen Bewältigung“ (Pfeiffer 2015, S. 23).

Es ist davon auszugehen, dass die Berufsbildung angesichts der Veränderungen relativ gut gerüstet ist. Bezogen auf die für erforderlich gehaltenen überfachlichen Qualifikationen lässt sich festhalten, dass sie bereits „heute fester Bestandteil von Metallund Elektroberufen“ (Ahrens/Spöttl 2015, S.198) sind. Einzelne Berufsbilder wie das 
des Produktionstechnologen bzw. der Produktionstechnologin, das allerdings bisher von den Betrieben und von den Jugendlichen kaum nachgefragt wird, enthalten schon heute wesentliche Ausbildungsinhalte zur Beherrschung systemischer Arbeitsprozesse. In einer umfangreichen Studie der Universität Bremen für die bayrischen Metallarbeitgeber wird auf der Basis einer mehrschichtigen empirischen Methode dieser Trend im Grundsatz bestätigt. So halten die in der Studie befragten Expertinnen und Experten neue Berufsbilder in der dualen Ausbildung für nicht erforderlich, aber es wird auf eine „Neuausrichtung mit einer Prozess- und Digitalisierungsperspektive“ (bayme vbm 2016, S. 3) verwiesen. Facharbeiter, Meister und Techniker haben demnach eine Beschäftigungschance in der digitalen Arbeitswelt, wenn sie die durch Digitalisierung hervorgerufenen Prozessabläufe „in ihrer Komplexität beherrschen und einen störungsfreien Betrieb von Anlagen sicherstellen können“ (ebd.). Dabei erscheinen insbesondere solche Bildungskonzepte besonders dringend zu sein, „die einerseits produkt- und prozessbezogen sind und andererseits auf Karriereförderung ausgerichtet sein sollen" (ebd., S. 5). Ausbildungsordnungen sind demnach anzupassen, es mögen auch neue Berufe notwendig werden. Weiterbildungsangebote für Facharbeiter und technische Fachkräfte sind erheblich auszuweiten. ${ }^{\prime}$

Auch auf die Ingenieurwissenschaften und die Informatik kommen große Herausforderungen zu. Ob nun allerdings die Stunde der Informatiker bzw. Informatikerinnen schlägt, wie es teilweise schon kolportiert wurde, ist (noch) nicht geklärt und möglicherweise auch ein Ergebnis arbeitspolitischer Aushandlung. Anstelle einer hierarchisierten Produktion, in der sich technische Fachkräfte als mögliche „Gewinner" und Facharbeiter sowie gering Qualifizierte als mögliche „Verlierer" gegenüberstehen, lässt sich auch eine kooperative, hierarchiearme Arbeitsorganisation vorstellen, in der gewinnbringend das Miteinander von Facharbeit und (produktionsnaher) Ingenieurarbeit geübt wird.

Wie im gewerblichen Bereich wird es auch in den technischen Studiengängen zu einer stärkeren Integration bisher getrennter ingenieurwissenschaftlicher Fächer, z. B. in der Zusammenführung von Wissensbeständen aus Anlagen- und Maschinenbau, Elektro- und Automatisierungstechnik und Informatik kommen. Auch wenn nicht zwangsläufig neue Studiengänge entstehen werden, so müssten doch „die Maschinenbauer in Informatik und die Informatiker in Maschinenbau" (VDI-Nachrichten 2014) qualifiziert werden. Es ist auch von einem Zuwachs der sog. überfachlichen Kompetenzen auszugehen. Die auf der Basis einer „Unternehmensbefragung“ agierende Studie des Stifterverbandes kommt zu dem Schluss, dass technische Studienprogramme um den Erwerb „berufsorientierter Kompetenzen“ in Bezug auf die An-

1 In einem Projekt, welches vom ITB koordiniert wird und an dem u. a. auch die IG Metall beteiligt ist, wird derzeit daran gearbeitet, arbeitsprozessorientierte Kompetenzentwicklung und wissenschaftliche Weiterbildung an der Schnittstelle von Facharbeit und Ingenieurtätigkeiten konzeptionell zusammenzuführen. Dazu werden modellhaft Konzepte des agilen Lernens, des Microlearning und des Lernbegleiterkonzeps weiterentwickelt (vgl. dazu www.brofessio.de). 
wendung von Fachwissen wie auch um sog. berufsorientierte „Digitalkompetenzen“ erweitert werden müssen (Stifterverband 2016, S. 27).

Das dazu notwendige Verhältnis von Theorie und Praxis im Lernprozess stellt sich im besten Fall in der Integration und nicht in der Addition von Methoden dar, d.h. nicht das Auslagern des Erwerbs von "Schlüsselqualifikationen“ in eigenständige Einrichtungen oder das Verdrängen der Praxis in das Praxissemester, sondern ein "Wechselspiel von praktischem Handeln und theoriegeleitetem Wissen“ (IG Metall 2014, S. 28) macht die neue Qualität wissenschaftlichen Lernens aus. Dieses Wechselspiel von Theorie und Praxis kann auf unterschiedlichen Wegen geschehen: Dazu gehören beruflich orientierte Aufgabenstellungen, etwa ausgewählt an künftigen oder im Betrieb erfahrener Arbeits- und Geschäftsprozesse, Studien- und Projektarbeiten, Lerngruppen, Exkursionen, Betriebserkundungen, Planspiele, Praktika und Praxisphasen. Das „Lernen im Arbeitsprozess“ an einem zweiten Lernort im Betrieb wird konstitutiv. Alle Praxisanteile - so eine Handlungshilfe der Gewerkschaften für die Akkreditierung und Studienganggestaltung von Ingenieurstudiengängen, „erfordern die explizite Formulierung von Kompetenzzielen oder -anforderungen im Hinblick auf das Erreichen beruflicher Handlungskompetenz" (Gewerkschaftliches Gutachternetzwerk 2009, S. 34).

Ein weiteres Thema bleibt die Bestimmung des Verhältnisses von Erfahrungs- und Wissenschaftsorientierung. In allgemeiner Weise ist die Formulierung im Leitbild unstrittig, dass „ein bloß kognitiver und/oder wissensbasierter Zugang (...) nicht aus(reicht), um berufliche Handlungskompetenzen zu entwickeln" (IG Metall 2014, S. 26). Ob es als gleiches, gleichwohl die Differenzen beider Bildungstypen akzeptierendes Prinzip für die betrieblich-duale Ausbildung wie für das Studium gelten kann, wird von WissenschaftlerInnen, die auch beratend an der Entstehung des Leitbildes beteiligt waren, kontrovers diskutiert. Georg Spöttl weist darauf hin, dass die „wissenschaftliche Ausbildung an Hochschulen (...) völlig anderen Handlungslogiken (folgt) als eine betrieblich-duale Ausbildung. In Hochschulen dominiert abstrakt-systematisches, wissenschaftliches Fachwissen, das mit wissenschaftlichen Methoden zu erschließen ist" (Spöttl 2015 ohne Seitenangabe). Entscheidend seien nicht die Methoden, sondern das Erkenntnisinteresse und die Unterschiedlichkeit der beruflichen Aufgaben. Jürgen Strauß - in derselben Veröffentlichung - leugnet die Unterschiede wie weiterhin notwendige Klärungen nicht. Für ihn steht auch nicht die Beschreibung des Status quo im Vordergrund, sondern ein Prozess des Voneinander-Lernens. „Das Leitbild kann eine Orientierung für die Reform hochschulischer und betrieblich-dualer beruflicher Bildung sein" (Strauß 2015 ohne Seitenangabe; vgl. Meyer 2016; Kutscha 2015).

\subsection{Beruflichkeit als Politikkonzept}

In einem ökonomischen und gesellschaftlichen Kontext von Deregulierung, Prekarisierung und zunehmender Vermarktlichung ist die Sicherung und Weiterentwicklung von Beruflichkeit ein zentrales Moment, um die Beschäftigungsperspektiven 
der Menschen zu verbessen, um über die Vermittlung zur Qualifizierung einen Beitrag zu guter, qualifikationsförderlicher und ganzheitlicher Arbeit zu leisten und um gesellschaftliche Integration zu fördern. Dafür entscheidend ist, dass das Leitbild letztendlich die Selektivität und Hierarchisierung zwischen allgemeiner und beruflicher Bildung infrage stellt.

Impulse lassen sich auf verschiedenen Ebenen ziehen. So geht es unmittelbar und vorrangig um die Qualität beruflicher Lernwege, also um die Weiterentwicklung von beruflichen Aus- und Fortbildungskonzepten, um die Reform von Studium, Lehre und wissenschaftlicher Weiterbildung. An den Schnittstellen von Berufsbildung und Hochschulsystem erscheinen die Möglichkeiten und Anforderungen an neue Lernwege. Sie müssen gewollt und die Wege durchlässig gestaltet werden. Beruflichkeit als Politikkonzept hat ihre Schnittstellen zur Arbeitspolitik, zur Betriebs- und Tarifpolitik, zur Gesellschaftspolitik. Indem gemeinsame Maßstäbe für berufliches Lernen in der dualen Ausbildung und im Studium, für Fortbildung und Weiterbildung formuliert werden, stellt es letztlich auch die Frage nach dem übergreifenden Zusammenhang der Bildungssysteme und damit nach der künftigen Bildungsarchitektur.

\section{Schlussfolgerungen für wissenschaftliche Weiterbildung}

Unbestritten ist, dass der Stellenwert wissenschaftlicher Weiterbildung an Hochschulen ausgebaut werden muss. Dafür fehlt noch immer eine Reihe von institutionellen und finanziellen Rahmenbedingungen. Vor allem muss eine finanzielle Grundausstattung bereitgestellt werden, welcher der wissenschaftlichen Weiterbildung den Druck der Vollkostenrechnung nimmt und damit den Trend zunehmender Kommerzialisierung relativiert. Die Arbeitsverhältnisse in den Einrichtungen müssen abgesichert werden. Die Lernenden sollen in den Genuss einer reformierten Studienförderung und verbesserter Stipendienmöglichkeiten kommen.

Gleichwohl ist auch wissenschaftliche Weiterbildung konzeptionell weiterzuentwickeln. Wissenschaftliche Weiterbildung - dies vorausgeschickt - hat einen breiteren Ansatz als den der Orientierung an beruflicher Qualifizierung. Nicht zufällig unterstützt die DGFW in ihrer Oldenburger Erklärung den Ausbau der wissenschaftlichen Weiterbildung für ältere Menschen während und nach der beruflichen bzw. familiären Tätigkeit. Es muss weiterhin allgemein- und kulturwissenschaftliche Themenfelder in wissenschaftlicher Weiterbildung geben. Wissenschaftliche Weiterbildung umfasst „berufliche, politische und allgemeine Bildung in einer Vielzahl von Veranstaltungsformen“ (DGWF 2010).

Offensichtlich muss sich wissenschaftliche Weiterbildung aufgrund veränderter Rahmenbedingungen auch dem Lernen in und für die Arbeitswelt in einer weiterentwickelten Weise zuwenden. Als Institution verfügt sie mit den Lehrenden und Lernenden sowie ihrem institutionellen Umfeld über spezifische Kompetenzen.

Zum Abschluss sollen drei Themen zusammenfassend herausgestellt werden. 


\subsection{Orientierung an beruflicher und wissenschaftlicher Handlungskompetenz}

Wissenschaftliche Weiterbildung, die sich weniger von der Institution und weniger von der in ihren Formaten strukturierten Angebotsseite her definiert, sondern von den Lernenden, ihren Erwerbs- und Lernbiografien und den institutionellen Nachfragen aus Gesellschaft und Wirtschaft, unterliegt der Gefahr der Beliebigkeit, wenn sie nicht aus der Institutionen selbst pädagogische, didaktische und curriculare Standards setzt. Sie wird jedoch immer schwieriger, je mehr Lernende die Erkenntnisse wissenschaftlicher Weiterbildung beruflich verwerten möchten oder müssen. Wissenschaftlichkeit als einziger Referenzpunkt reicht in diesem Kontext nicht. Der Bezug allein auf die Anforderungsseite des Arbeitsmarktes macht nicht nur wissenschaftliche Weiterbildung als Institution angreifbar, sondern ist auch mit einem weitgehenden Verlust eines Bildungsanspruches verbunden. Lässt sich der wissenschaftsbezogene Anspruch dagegen mit Beruflichkeit verbinden, so könnten Maßstäbe einer an der beruflichen Qualifizierung orientierten wissenschaftlichen Weiterbildung gewonnen werden. Neben der Orientierung an fachwissenschaftlichen Erkenntnissen und Fähigkeiten tritt ein an den Berufsfeldern der Teilnehmenden orientiertes Verständnis von wissenschaftlicher und beruflicher Handlungskompetenz. Dieses ist der Kern eines sich an zentralen Arbeits- und Geschäftsprozessen orientierenden und auf einer spezifischen Vermittlung von Beruflichkeit und Wissenschaftlichkeit aufbauenden Bildungskonzeptes, aus dem heraus sich auch beruflich-fachliche Standards ableiten lassen, die der Institution der wissenschaftlichen Weiterbildung auch ein Alleinstellungsmerkmal gegenüber anderen Wettbewerbern geben können.

\subsection{Anforderungen nach mehr sozialer und beruflicher Durchlässigkeit}

Wissenschaftliche Weiterbildung, die sich mehr von den Erwerbs- und Lernbiografien her definiert als von den wissenschaftlichen Gegenständen der jeweiligen Fächer, muss nicht nur in der Lage sein, wissenschaftliche Erkenntnisse an den Berufsfeldern orientiert fächerübergreifend $\mathrm{zu}$ organisieren. Sie muss auch Abschied nehmen vom Nachweis eines Studienabschlusses als einer zentralen Teilnehmervoraussetzung. Wissenschaftliche Weiterbildung sollte daher durchlässig werden für Berufserfahrene mit und ohne Hochschulabschluss. Damit ist ganz elementar die Anforderung verbunden, curricular an den Lern- und Arbeitserfahrungen der Lernenden anzusetzen. Aus Sicht der berufserfahrenen Lernenden kann dabei selbst das grundständige Bachelorstudium eine Weiterbildung sein. Entsprechend müssten sich die Institutionen der wissenschaftlichen Weiterbildung in der Hochschule als Kompetenzzentrum für die Integration von Berufserfahrenen einbringen können. Auch für wissenschaftliche Weiterbildung stellt sich zudem die Frage der sozialen Durchlässigkeit. Dies beginnt bei den Gebühren, die Teilnehmende oft nicht aufbringen können, Studienförderung, für die keine Ansprüche bestehen, Beratungsangebote, die nicht existieren oder nicht zielgruppengerecht aufbereitet sind, 
und endet bei Angeboten, die keine Brücken zu Arbeits- und Lernerfahrungen dieser möglichen Teilnehmerinnen und Teilnehmer bauen.

\subsection{Wissenschaftliche Weiterbildung als Teil der Bildungsarchitektur}

$\mathrm{Ob}$ die Diskussion über die vielfältigen Implikationen der Akademisierung, die aus dem demografischen Wandel resultierende Anforderung an die Sicherung des Fachkräftebedarfs oder die Frage nach notwendigen und sinnvollen Arbeits- und Qualifikationskonzepten im Zuge der zunehmenden Digitalisierung - insgesamt führen diese Entwicklungen zu einer neuerlichen und notwendigen Diskussion über das Verhältnis von allgemeiner und beruflicher Bildung. Ein hierarchisch gegliedertes Bildungssystem ist ebenso überholt wie die im Bildungsschisma angelegte systematische Trennung von Allgemein- und Berufsbildung. Aktuell werden aufgrund der veränderten Rahmenbedingungen interessante Debatten geführt, wie mehr gegenseitige Durchlässigkeit geschaffen und neue Lernwege zwischen den Teilsystemen angeboten werden und zu größeren Schnittmengen führen können. Teile der beruflichen Bildung diskutieren für die Ausbildungsberufe neue Berechtigungen als „Berufsabitur" und bauen über Zusatzqualifikationen Brücken ins Hochschulstudium. Der Wissenschaftsrat fordert die Hochschulen und das Berufsbildungssystem auf, mehr „hybride“ Studienangebote zu schaffen. Es gibt ernsthafte und hoffnungsvolle Ansätze der „offenen“ Hochschule, positive Erfahrungen im zweiten und dritten Bildungsweg. Es gibt Denkanstöße für eine andere „Versäulung“ von hochschulischer und beruflicher Bildung; es gibt Modelle der Integration von hochschulischer und beruflicher Bildung. Eines haben diese Prozesse gemeinsam: Sie rufen die Frage auf, wie künftig die Bildungsarchitektur beschaffen und über welche Instrumente sie debattiert werden kann, etwa über die Einrichtung einer Bildungskommission, wie sie der DGB fordert.

Wissenschaftliche Weiterbildung muss sich in diese Debatten eindenken und positionieren. Hierbei wird sicherlich der Strukturwandel des tertiären Bereichs eine wesentliche Markierung sein; allein diese Raumbegrenzung reicht nicht. Darüber hinaus wird auch der Zusammenhang zum großen Feld der beruflichen, allgemeinen und politischen Weiterbildung wie das Verhältnis von Hochschul- und Berufsbildung geklärt werden müssen. Aus Sicht der Gewerkschaften gäbe es dafür einen Maßstab, den Peter Faulstich in einem Beitrag vor dem Bildungsausschuss der IG Metall so in Worte gefasst hat:

„Wenn wir eine Bildungs- und Arbeitspolitik in Richtung auf einen Integrationsprozess wollen, der die bisherige Trennung selektiver Bildungsinstitutionen und polarisierter Arbeitseinsatztypen überwindet, muss auch die Polarität von ,akademischen und ,betrieblichem' Bildungstyp aufgehoben und müssen die traditionellen Barrieren zwischen Facharbeitern und Akademikern aufgebrochen werden, die sich über hierarchische Abschlüsse definieren“ (Faulstich 2015). 


\section{Literatur}

Ahrens, D./Spöttl, G. (2015): Industrie 4.0 und Herausforderungen für die Qualifizierung von Fachkräften. In: Hirsch-Kreinsen u. a. (Hrsg.): Digitalisierung industrieller Arbeit. Baden-Baden, S. 185-205.

bayme vbm (Bayrischer Unternehmensverband Metall und Elektro e. V./Verband der Bayrischen Metall- und Elektroindustrie) (Hrsg.) (2016): Industrie 4.0 - Auswirkungen auf Aus- und Weiterbildung in der M+E Industrie, April 2016.

BMBF (2016): Wissenschaftliche Weiterbildung. www.bmbf.de/de/wissenschaftliche-wei terbildung-1311.html

Bosch, G. (2016): Weiterbildung in der Sackgasse. In: VDI-Nachrichten vom 27.05.2016.

Dengler, K./Matthes, B. (2015): Folgen der Digitalisierung der Arbeitswelt, IAB - Forschungsbericht 11/2015, Nürnberg, Dez. 2015.

DGB Bundesvorstand (Hrsg.): Für eine demokratische und soziale Hochschule. Das hochschulpolitische Programm des Deutschen Gewerkschaftsbundes, Dezember 2012.

DGWF (2010): DGWF-Empfehlungen zu Formaten wissenschaftlicher Weiterbildung. www.dgwf.net

Faulstich, P. (2010): Von der „Universitären Erwachsenenbildung“ zur „Wissenschaftlichen Weiterbildung“. In: Hochschule und Weiterbildung, S. 30-35.

Faulstich, P./Oswald, L. (2012): Wissenschaftliche Weiterbildung. In: Hans-Böckler-Stiftung (Hrsg.): Expertisen für die Hochschule der Zukunft. Demokratische und soziale Hochschule. Bad Heilbrunn, S. 373-399.

Faulstich, P. (2015): Konvergenzstrategie für eine gemeinsame Weiterentwicklung betrieblicher, schulischer und hochschulischer Berufsbildung, Vortrag vor dem Bildungsausschuss der IG Metall vom 21. Mai 2015. www.wap.igmetall.de

Freitag, W.K/Buhr, R./Danzeglocke, E.-M./Schröder, S./Völk, D. (Hrsg.) (2015): Übergänge gestalten. Durchlässigkeit zwischen beruflicher und hochschulischer Bildung erhöhen. Münster.

Frey, C. B./Osborne, M. (2013): The Future of Employment. Oxford.

Gewerkschaftliches Gutachternetzwerk (Hrsg.) (2009): Studium als wissenschaftliche Berufsausbildung. Oktober 2009. www.gutachternetzwerk.de

Hanft, A./Brinkmann, K./Kretschmer, S./Maschwitz, A./Stöter, J. (2016): Organisation und Management von Weiterbildung und Lebenslangem Lernen an Hochschulen. Ergebnisse der wissenschaftlichen Begleitung des Bund-Länder-Wettbewerbs „Aufstieg durch Bildung: offene Hochschulen“. Band 2. Münster.

IG Metall (2014): Erweiterte moderne Beruflichkeit. Ein gemeinsames Leitbild für die betrieblich-duale und die hochschulische Berufsbildung. Diskussionspapier. November 2014.

IG Metall (2016): Digitalisierung der Arbeitswelt - Herausforderungen für die Berufsbildung. Ein Thesenpapier. 16.06.2016. https://wap.igmetall.de/15843.htm 
Kamm, C./Schmitt, S./Banscherus, U./Wolter, A. (unter Mitarbeit von Golubchykova, O. (2016): Hochschulen auf dem Weiterbildungsmarkt. In: Wolter, A./Banscherus, U./ Kamm, C. (Hrsg.): Zielgruppen Lebenslangen Lernens an Hochschulen. Ergebnisse der wissenschaftlichen Begleitung des Bund-Länder-Wettbewerbs Aufstieg durch Bildung: offene Hochschule. Band 1 Münster, New York, S. 137-165.

Kuda, E./Strauß, J./Spöttl, G./Kaßebaum, B. (Hrsg.) (2012): Akademisierung der Arbeitswelt? Zur Zukunft der beruflichen Bildung. Hamburg.

Kutscha, G. (2015): Erweiterte moderne Beruflichkeit - eine Alternative zum Mythos „Akademisierungswahn“ und zur „Employability-Maxime“ des Bologna-Prozesses. In: bwp@ Berufs- und Wirtschaftspädagogik online, 29. http://www.bwpat.de/ausgabe/29/ kutscha

Meyer, R. (2016): Da bewegt sich mehr als man denkt ... und doch noch zu wenig. Zum Verhältnis von beruflicher und hochschulischer Lernkultur. In: www.denk-dochmal.de. Das Online Magazin, 2016(1).

Pasternack, P./von Wissel, C. (2012): Programmatische Konzepte der Hochschulentwicklung in Deutschland seit 1945. In: Hans-Böckler-Stiftung (Hrsg.): Expertisen für die Hochschule der Zukunft. Bad Heilbrunn, S. 21-72

Pfeiffer, S. (2015): Auswirkungen von Industrie 4.0 auf Aus- und Weiterbildung. Wien, Oktober 2015 .

Spöttl, G. (2015): Einspruch aus der Hochschule - welche Bedenken gibt es? In: www.denk-doch-mal.de. Das Online Magazin, 2015(1).

Stifterverband (Hrsg.) (2016): Hochschulbildung für die Arbeitswelt 4.0. www.hochschul bildungsreport2020.de

Strauß, J. (2015): Erfahrungs- und Wissenschaftsorientierung - gemeinsame und unterschiedliche Prinzipien des Lernens in betrieblich-dualer Ausbildung und im Studium. In: www.denk-doch-mal.de. Das Online Magazin, 2015(1).

Verdi/IG Metall (Hrsg.) (2014): Wissenschaftlicher Beraterkreis, Leitlinien für eine gemeinsame duale, schulische und hochschulische berufliche Bildung, April 2014.

Wolter, A. (2011): Die Entwicklung wissenschaftlicher Weiterbildung in Deutschland: von der postgradualen Weiterbildung zum Lebenslangen Lernen. In: Beiträge zur Hochschulforschung, 2011(4), S. 8-35.

Wolter, A. (2016): Die Heterogenität beruflich Qualifizierter im Hochschulstudium. Aktuelle Forschungsergebnisse zur Teilnahme an Hochschulweiterbildung. In: Hochschule und Weiterbildung, 2016(1), S. 9-19.

Wolter, A./Banscherus, U./Kamm, C. (Hrsg.) (2016): Zielgruppen Lebenslangen Lernens an Hochschulen. Ergebnisse der wissenschaftlichen Begleitung des Bund-Länder-Wettbewerbs „Aufstieg durch Bildung: offene Hochschulen“. Band 1. Münster. 


\title{
Zielgruppen in der wissenschaftlichen Weiterbildung
}

WOLFGANG SEITTER

\begin{abstract}
Der Aufsatz bestimmt Zielgruppen als ein Resultat vielfältiger Passungsprozesse zwischen Angebot und Nachfrage. Nach einer systematischen Klärung dieser Passungsherausforderungen werden am Beispiel der abschlussorientierten wissenschaftlichen Weiterbildung zwei große Zielgruppenbereiche differenziert dargestellt, um dann die Bedeutung hochschulinterner Zielgruppen für ein erfolgreiches Matching von Angebot und Nachfrage herauszustellen.
\end{abstract}

Der Zielgruppenbegriff ist ein relationaler Begriff, der auf eine Verhältnisbestimmung und möglichst zielgenaue Passung von (geplantem) Angebot und (intendierter) Nachfrage abhebt. Dabei lässt sich der Zielgruppenbegriff stärker von der Angebots- und Organisationsseite oder von der Nachfrage- und Adressatenseite her denken. Je nach gewähltem Fokus sind die Ausgangsfragen und Systematisierungsprozesse (zunächst) verschieden, entscheidend ist allerdings ihr wechselseitiger Bezug. Was unter wissenschaftlicher Weiterbildung je konkret gefasst wird, auf welches Spektrum von Angebotsvarianten wissenschaftlicher Weiterbildung Adressaten und Zielgruppen bezogen werden (können und sollen), welche Personengruppen innerhalb der Hochschulen für welche Varianten überhaupt zur Verfügung stehen, welche angebotsbezogenen Voraussetzungen Zielgruppen erfüllen müssen, nach welchen Differenzierungskriterien sich Zielgruppen beschreiben lassen - all dies sind Fragen, die nicht unabhängig voneinander, sondern in einer engen Wechselbeziehung zueinander geklärt und beantwortet werden müssen. Zielgruppen konstituieren sich insofern nicht als Resultante unterschiedlicher (isolierter) Bezugsgrößen, sondern vielmehr als Resultat unterschiedlicher Relationierungen von Bezugsgrößen (Angebotssegmente und -varianten, individuelle und institutionelle Adressaten, externe und interne Zielgruppen). Daher geht es bei Prozessen der Zielgruppenkonstitution immer um Passungs-, Übersetzungs- und Matchingherausforderungen, die spezifische - wie auch immer zu beschreibende - Personengruppen mit einem spezifischen Angebot relationieren (wollen). 
Im Folgenden wird zunächst eine systematisch-konzeptionelle Klärung dieser Passung(sherausforderungen) vorgenommen (1), um dann zwei dominante Zielgruppen der abschlussorientierten wissenschaftlichen Weiterbildung näher zu beschreiben (2). Abschließend wird die Passungsnotwendigkeit von externen und internen Zielgruppen der wissenschaftlichen Weiterbildung herausgestellt (3). Ein kurzes Fazit fasst die zentralen Ergebnisse zusammen und gibt einen fokussierenden Ausblick (4).

\section{Zielgruppe als konzeptioneller Passungsbegriff zwischen Angebot und Nachfrage}

Der Zielgruppenbegriff in der Weiterbildung ist ein relationaler Begriff, da er nicht unabhängig von spezifischen Angebotskonzeptionierungen gedacht werden kann. Angebote bzw. Angebotsvarianten präjudizieren die Zielgruppenbestimmung wie auch umgekehrt die antizipierte Zielgruppenbestimmung das bereits existente oder potenzielle Angebot konkretisiert bzw. modifiziert. Zudem sind Angebote in der wissenschaftlichen Weiterbildung und darauf bezogene Zielgruppenbestimmungen stark abhängig von hochschulrechtlichen und länderspezifischen Rahmenbedingungen. Was für wen ein Angebot wissenschaftlicher Weiterbildung darstellt, ist je unterschiedlich zu beantworten, je nachdem, ob man die Sicht der Adressaten oder die Sicht der hochschulischen und hochschulrechtlich gebundenen Angebotsseite als Differenzierungskriterium nimmt. So kann ein im hochschulrechtlichen Sinne als grundständig definierter Studiengang aus der bildungs- und berufsbiografischen Sicht von Teilnehmenden ein weiterbildendes (Erst-)Studium sein, wobei sich bereits die Nomenklatur in den länderspezifischen Regelungen zum Teil deutlich voneinander unterscheidet. Die vielfältigen Charakterisierungen von Studienangeboten wie Vollzeit, Teilzeit, berufsbegleitend, berufsintegriert, dual, grundständig, weiterbildend, etc. erschweren klare Zuordnungen und markieren zum Teil deutliche Differenzen zwischen einer Angebots- und Finanzierungslogik und einer bildungsbiografisch verankerten Teilnahme- und Zielgruppenlogik.

Neben diesen eher systemisch bedingten hochschulpolitischen, -rechtlichen und -finanziellen Implikationen des Zusammenhangs von Angebot und Zielgruppenbestimmung ist mit Blick auf die erwachsenenpädagogisch-programmplanerische Ebene die Differenzierung von Adressaten, Teilnehmenden und Zielgruppen von Bedeutung. Zielgruppenbestimmungen sind nicht losgelöst von didaktischen Programmplanungsvorstellungen durchzuführen. Während der Adressatenbegriff in der Weiterbildung auf (zunächst) unspezifizierte potenzielle Teilnehmende abzielt, impliziert der Zielgruppenbegriff eine nach bestimmten Kriterien vorgenommene Spezifizierung des Adressatenbezugs. Diese Spezifizierung wird in der Regel vor dem Hintergrund der eigenen - bereits vorhandenen bzw. neu zu entwickelnden Bildungsangebote vorgenommen. Betrachtet man in dieser Perspektive die Angebotspalette dessen, was als wissenschaftliche Weiterbildung bezeichnet werden kann, so lassen sich die bisher entwickelten Formate nach Kriterien differenzieren 
wie Abschlussorientierung (mit oder ohne Zertifikat), Zeitdauer (lang oder kurz), inhaltliche Ausrichtung (Forschungs-, Berufs- oder Allgemeinbildungsbezug), Zugänglichkeit (offen für alle oder exklusive Zugangskriterien) oder Finanzierungsform (unentgeltlich ${ }^{1}$ oder kostenpflichtig). Für die mit dem Angebot korrespondierende Zielgruppenkonstitution macht es daher einen zentralen Unterschied aus, ob das Angebot mit Zertifikat endet, von langer Dauer ist, einen klaren Berufsbezug aufweist, an bestimmte Zugangskriterien gebunden ist und vollkostendeckend durch Teilnahmeentgelte finanziert wird, oder ob das Angebot ohne Zertifikatsabschluss, mit kurzer Zeitdauer, allgemein zugänglich und unentgeltlich durchgeführt wird. Umgekehrt kann eine vorgelagerte - das bisherige Angebot zunächst einklammernde - Zielgruppenkonstitution bzw. manifeste Nachfrage deutliche Auswirkungen auf das Angebot haben, im Sinne einer Anpassung, Modifizierung, Erweiterung, Neukombination oder Neuschöpfung von Elementen - allerdings immer im Rahmen der organisationsstrukturellen und -kulturellen Möglichkeiten der anbietenden Hochschule.

Als Resultat bisheriger Suchbewegungen und Passungsprozesse sind bestimmte Korrespondenzen zwischen Angeboten wissenschaftlicher Weiterbildung und Zielgruppen innerhalb der Hochschulen bereits seit vielen Jahren fest institutionalisiert, etwa nach Alter (Seniorenstudium, Kinderuniversität), nach Status (Gasthörerinnen und -hörer) oder nach öffentlichkeitsbezogenen Formaten (Bürgervorlesung, Studium generale). Diese Korrespondenzen können auch organisationsstrukturelle Segmentierungen annehmen (Universität des dritten Lebensalters, Weiterbildungszentren, weiterbildungsspezifische Ausgründungen) oder die Hochschule als Typ insgesamt prägen (Open Universities, Weiterbildungsuniversitäten). Weitere zielgruppenorientierte Konstruktions- und Beschreibungsprinzipien unterscheiden nach soziodemografischen, psychografischen oder verhaltensorientierten Kriterien (vgl. Haubenreich/Breitenberger 2015) oder setzen die „klassischen“ Studierenden jüngeren Alters im Modus des grundständigen Vollzeitstudiums von den sog. „nontraditional students“ oder „lifelong learners“ ab. So weisen etwa Wolter und Banscherus (2016, S.70 ff.) in Anlehnung an internationale Unterscheidungen eine Typologie erwachsener Lernender an Hochschulen aus, die je nach Hauptdifferenzkriterium ihr (weiterbildendes) Studium über alternative Zugangswege beginnen (second learners), unterrepräsentierten Bevölkerungsgruppen angehören (equity groups), trotz formaler Zugangsberechtigung ihr Studium mit zeitlicher Verzögerung beginnen (deferrers), sich erneut wissenschaftlich weiterqualifizieren (recurrent learners), das Studium nach Unterbrechung wieder aufnehmen (returners), ihre Wissensbestände auffrischen (refreshers) oder erst in der nachberuflichen Phase an die Hochschule gehen (learners in later life). Weitere zielgruppenspezifische Einteilungen orientieren sich an der dominanten Inhaltsausrichtung der Angebote und unterscheiden nach Professions- oder Funktionsorientierung (vgl. Weber

1 Unentgeltlich bezieht sich hier lediglich auf die Kostenfreiheit für die Teilnehmenden. Für die anbietenden Hochschulen entstehen selbstverständlich auch für derartige Angebote z. T. erhebliche Kosten. 
2010). Eine überaus differenzierte, nach didaktischen Ebenen sortierende Matrix von Koppelungen und didaktischen Relationierungen legen Walber und Jütte (2015) vor. Im Segment der abschlussorientierten, längerfristigen Weiterbildung werden schließlich individuelle und institutionelle Zielgruppen unterschieden und der doppelte Zielgruppenbezug wissenschaftlicher Weiterbildung herausgestellt (vgl. Seitter 2014). Adressaten und Zielgruppen sind in diesem Segment häufig in Arbeitskontexte von Einrichtungen und Betrieben eingebunden, die oft darüber (mit-)bestimmen, ob und wie die Weiterbildungsteilnahme inhaltlich, zeitlich und finanziell unterstützt wird. Daher ist gerade in diesem Segment eine ansonsten häufig anzutreffende individualistische Fokussierung auf Einzelpersonen wenig zielführend, sondern vielmehr von einem doppelten - durchaus schwierig auszubalancierenden - Zielgruppenbezug auszugehen.

Insgesamt zeigen sich sehr unterschiedliche Ausprägungsformen der Zielgruppenkonstitution, wobei die Differenzierungskriterien vornehmlich aus vorgelagerten gesetzgeberischen Bestimmungen (Hochschulrecht), aus didaktisch-handlungspraktischen Erwägungen erfolgreicher Ansprache und Passung (Weiterbildungspraxis) oder aus empirisch-systematischen Ordnungsversuchen (Forschung) resultieren.

\section{Zielgruppen der abschlussorientierten Weiterbildung}

Im Folgenden soll nicht auf die große Fülle möglicher - und auch faktisch vorhandener - Zielgruppen der wissenschaftlichen Weiterbildung eingegangen werden, wie sie etwa im Bereich der allgemeinen öffentlichkeitsbezogenen Angebote (Studium generale, Bürgeruniversität), der lebensphasenbezogenen Formate (Seniorenstudium, Kinder- und Schüleruniversität) oder der Studienvarianten von nicht traditionell Studierenden im grundständigen Bereich des akademischen Erstabschlusses (zweiter oder dritter Bildungsweg, lebensphasenverzögerte Studienaufnahme, berufsbegleitendes Studium, etc.) zu finden sind. Vielmehr werden die beiden großen Zielgruppenbereiche im spezifischen Segment der abschlussorientierten wissenschaftlichen Weiterbildung (Bachelor, Master, Zertifikate) fokussiert, die bildungspolitisch derzeit besondere Beachtung finden: die Gruppe der bereits akademisch Qualifizierten, die sich erneut wissenschaftlich weiterbildet, und die Gruppe der beruflich Qualifizierten ohne traditionelle Hochschulzugangsberechtigung, die über die wissenschaftliche Weiterbildung zum ersten Mal an hochschulischen Angeboten teilhat. Beide Gruppen werden vor dem Hintergrund gesellschaftlicher Megatrends (demografischer Wandel, Fachkräftemangel, Wissensbasierung von Arbeit, etc.) gegenwärtig besonders adressiert.

Die erste Gruppe der akademisch Qualifizierten, die nach einer Phase der Berufstätigkeit erneut an die Hochschule zurückkehrt, kann als klassische Zielgruppe der wissenschaftlichen Weiterbildung gelten. Sie ist schon seit vielen Jahren an den Hochschulen präsent und entspricht einem etablierten - organisationskulturell insbesondere von Universitäten am ehesten akzeptierten - Angebots- und Adressaten- 
schema (Akademikerinnen und Akademiker, die über formalisierte Studienangebote akademisch weitergebildet werden). Der Bologna-Prozess und die Stufung von Studiengängen hat dieses Format insofern weiter gestärkt, als berufstätige Bachelorabsolventen und -absolventinnen und die sie beschäftigenden Einrichtungen wissenschaftliche Weiterbildung zunehmend als eine Möglichkeit individueller und einrichtungsbezogener Weiterbildung sehen und auch vermehrt nachfragen. Zusammen mit anderen Faktoren wie den Notwendigkeiten systematischer Personalentwicklung und -bindung oder der zunehmenden Verwissenschaftlichung von Arbeitsprozessen hat dieses Segment in den letzten Jahren daher stark an Bedeutung gewonnen und eine große Varianz und Heterogenität entsprechender Zielgruppenorientierungen hervorgebracht. Die Zielgruppenbestimmungen lassen sich unterscheiden nach Branchen, Professionen, Hierarchiestufen oder Funktionen, aber auch nach gezielter interdisziplinärer Zusammensetzung entsprechender Berufsgruppen bei komplexen Herausforderungen (Umwelt, Energie, Mobilität, Cybersicherheit, Innovation, Baubegleitung, etc.). Abschlüsse, die in diesem Segment der wissenschaftlichen Weiterbildung erworben werden, können zudem häufig mit bereits bestehenden professionsorientierten Qualifikationssystemen (Ärzte-, Anwaltskammern) verbunden werden.

Die zweite Gruppe der nicht-traditionellen Studierenden, die mit Berufserfahrung und - meist - ohne traditionelle Hochschulzugangsberechtigung zum ersten Mal ein Studium absolviert, stellt die Hochschulen vor neue Herausforderungen. Bei diesem Zielgruppensegment geht es nicht nur um Fragen der berufsbegleitenden Studienorganisation, sondern auch um Probleme der Durchlässigkeit von beruflicher und akademischer Bildung, der Anrechnung und Anerkennung außerhochschulisch erworbener Kompetenzen oder der Übergangsgestaltung in ein akademisches Studium hinein. Dieser Zielgruppenbereich ist vor allem an Universitäten relativ neu und wird stark durch die bildungspolitische Diskussion um die Öffnung der Hochschulen geprägt. ${ }^{2}$ Auch in diesem Segment gibt es vielfältige zielgruppenspezifische Differenzierungen, die in etlichen Bereichen mit großen Hoffnungen einer zunehmenden akademischen Absicherung und Professionalisierung der eigenen Arbeit (Frühpädagogik, Pflege, Gesundheit) verbunden werden.

Beide großen Zielgruppenbereiche der abschlussbezogenen wissenschaftlichen Weiterbildung zeichnen sich einerseits durch umfassende vorgelagerte Zielgruppenanalysen und Bedarfserhebungen, andererseits durch didaktisch-lernkulturelle Anpassungen mit Blick auf Studienorganisation (Zeitregime), Beratung und Begleitung oder Studienvorbereitung (Brückenkurse) aus. In der Regel wird die zielgruppenspezifische Umsetzung auch von empirisch-evaluativ ausgerichteter Begleitforschung flankiert, um etwa Faktoren wie Studienerfolg, Zeitverausgabung, Lerntransfer, etc. genauer bestimmen zu können (vgl. dazu etwa die entsprechenden Studien und Übersichten in Wolter/Banscherus/Kamm 2016). In beiden Bereichen wird die

2 Das BMBF-Förderprogramm „Aufstieg durch Bildung: offene Hochschulen“ ist ein prominentes Beispiel für diesen bildungspolitischen Kontext. 
Komplexität der Zielgruppenbestimmung noch dadurch erhöht, dass neben den individuellen Adressaten zusätzlich auch die Belange der Einrichtungen/Unternehmen als institutionellen Adressaten berücksichtigt werden müssen, die durch Kostenübernahme und Freistellung die Teilnahme mitfinanzieren.

\section{Matching zwischen externen und internen Zielgruppen als Herausforderung der wissenschaftlichen Weiterbildung}

Für den Erfolg bei der Stärkung der Weiterbildungsfunktion von Hochschulen und einer entsprechenden lebenslauforientierten Ausrichtung ist das Selbstverständnis und die Akzeptanz des Hochschulpersonals sowohl in wissenschaftlicher wie in administrativer Hinsicht von entscheidender Bedeutung. In dieser Perspektive sind die Mitarbeitenden in Wissenschaft und Verwaltung ebenfalls (interne) Zielgruppen wissenschaftlicher Weiterbildung, die für diese (neue) Aufgabe ebenso gewonnen werden müssen wie externe Zielgruppen. Die Planungsverantwortlichen an Hochschulen für die wissenschaftliche Weiterbildung befinden sich insofern in der überaus herausfordernden Situation, nicht nur die externen, sondern auch und gerade die internen Zielgruppen für die Beteiligung an wissenschaftlicher Weiterbildung gewinnen zu müssen. Über Motive, Anreizfaktoren, Beteiligungsformen, etc. dieser beiden internen Zielgruppen gibt es bislang nur wenige empirische Befunde, wie auch die weitere zielgruppenbezogene Differenzierung dieser beiden Gruppen noch aussteht - etwa mit Blick auf Hierarchieebenen und Fächer(cluster), auf (mentale) Nähe oder Distanz, auf aktive Beteiligung, passive Duldung oder mehr oder weniger offene Verweigerung (vgl. Kahl/Lenger/Präßler 2015; Kloke/Krüken 2010).

Neben der Gewinnung dieser internen Zielgruppen liegt eine weitere Herausforderung für die Weiterbildungsverantwortlichen in dem Passungsprozess zwischen den verschiedenen internen und externen Zielgruppen. Es ist wenig sinnvoll, wissenschaftliches Personal mit einer ausgeprägten Forschungsorientierung auch in der wissenschaftlichen Weiterbildung für eine Zielgruppe mit einer ausgeprägten berufspraktischen Verwertungsorientierung gewinnen zu wollen. Dieser wechselseitige Passungs-, Übersetzungs- und Matchingprozess stellt die Akteure und Promotoren der wissenschaftlichen Weiterbildung vor deutlich größere Herausforderungen als Programmplanende in anderen organisationalen Settings der Weiterbildung (vgl. Schemmann/Seitter 2014). In diesem Matching-Prozess bilden sich überhaupt erst die Möglichkeitskorridore heraus, welche Zielgruppen innerhalb einer Hochschule realistischerweise adressierbar sind. Erste systematische Überlegungen und empirische Befunde für diese Matchingproblematik finden sich bei Seitter, Schemmann und Vossebein (2015, S. 48 ff.), die herausarbeiten, wie die unterschiedlichen Präferenzen für bestimmte Formen der wissenschaftlichen Weiterbildung miteinander kompatibel gemacht werden können. 


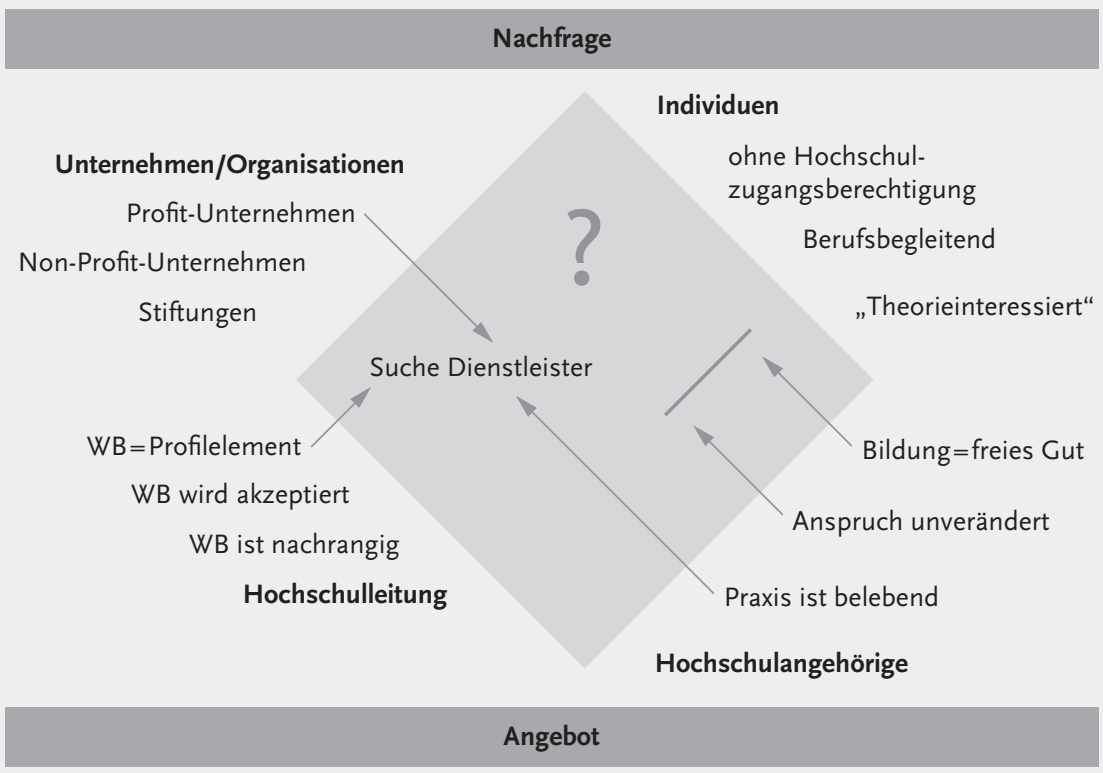

Abb. 1: Mehrdimensionales Beziehungsgeflecht zwischen den vier Zielgruppen (Seitter/Schemmann/Vossebein 2015, S. 56)

Erwarten beispielsweise gewinnorientierte Unternehmen - stärker als Non-ProfitEinrichtungen oder Stiftungen - eine effiziente, bedarfsgerechte Dienstleistungsorientierung der Hochschulen mit Blick auf Konzeptionierung, Umsetzung und Abwicklung von Studienangeboten, so ist es notwendig, dass nicht nur die Hochschulleitung die wissenschaftliche Weiterbildung als entsprechendes Profilelement definiert, sondern sich auch Hochschulangehörige sowohl auf der Wissenschaftswie auf der Verwaltungsebene finden, die einer derartigen Erwartungshaltung von Serviceorientierung tatsächlich entsprechen wollen und beispielsweise nicht darauf beharren, Bildung als ein kostenfreies öffentliches Gut zu verstehen. Erst in der erfolgreichen Passung dieser zielgruppenspezifischen Perspektiven entsteht der organisationale Möglichkeitskorridor erfolgreicher wissenschaftlicher Weiterbildung.

\section{Fazit und Ausblick}

Zielgruppen in der wissenschaftlichen Weiterbildung lassen sich unter verschiedenen Gesichtspunkten mit den damit verbundenen - je unterschiedlichen - Herausforderungen systematisieren und differenzieren:

- als Differenzierung mit Blick auf spezifische Angebotssegmente der wissenschaftlichen Weiterbildung und damit als Passungsherausforderung von Programmsegmenten und Zielgruppen; 
- als weitergehende segmentspezifische Differenzierung nach Branchen, Professionen, Hierarchiestufen oder Funktionen mit entsprechender Angebotskonkretion;

- als Differenzierung zwischen individuellen und institutionellen Zielgruppen und dem damit verbundenen doppelten Adressaten-/Zielgruppenbezug der Weiterbildung;

- als Differenzierung von externen und internen Zielgruppen mit der Betonung von Wissenschaft und Verwaltung als bedeutsame interne Zielgruppen;

- als Relationierung und wechselseitige Bezugnahme der internen und externen Zielgruppen mit der dazugehörigen Herausforderung eines dauerhaften, passgenauen Matching.

Eben dieser letzte Punkt komplexer Matchingprozesse zwischen den unterschiedlichen internen und externen Zielgruppen mit Blick auf ein spezifisches Angebot kommt in der bisherigen Diskussion deutlich zu kurz. Erst die gleichzeitige und iterative Berücksichtigung aller beteiligten Gruppierungen macht die erfolgreiche Umsetzung wissenschaftlicher Weiterbildung passgenauer und damit wahrscheinlich(er). Daher ist von einem Zielgruppenbegriff abzusehen, der den Fokus zu stark auf externe Adressatengruppen lenkt, ohne gleichzeitig die starke Abhängigkeit von und die Wechselwirkung mit internen Zielgruppen und den Möglichkeitskorridoren hochschulischer Angebotsgestaltung in den Blick zu nehmen.

\section{Literatur}

Haubenreich, J./Breitenberger, I. (2015): Zielgruppen im Projekt mint.online. Abschlussbericht zur Entwicklung einer zielgruppenorientierten Ansprache für Weiterbildungsstudierende in MINT-Fächern. http://www.c3l.uni-oldenburg.de/mintonline/files/ publikationen/Abschlussbericht_Zielgruppen\%2omint.online_2015.pdf

Kahl, R./Lengler, A./Präßler, S. (2015) (unter Mitarbeit von F. Lutzmann): Akzeptanzanalyse. Forschungsbericht zur Akzeptanz innerhochschulischer Zielgruppen: Verwaltungspersonal, wissenschaftliches Personal. In: Seitter, W./Schemmann, M./Vossebein, U. (Hrsg.): Zielgruppen in der wissenschaftlichen Weiterbildung. Empirische Studien zu Bedarf, Potential und Akzeptanz. Wiesbaden: Springer VS, S. 291-408.

Kloke, K./Krüken, G. (2010): Grenzstellenmanager zwischen Wissenschaft und Wirtschaft? Eine Studie zu Mitarbeiterinnen und Mitarbeitern in Einrichtungen des Technologietransfers und der wissenschaftlichen Weiterbildung. In: Beiträge zur Hochschulforschung, 32(3), S. 32-52.

Schemmann, M./Seitter, W. (2014): Angebotsentwicklung in der wissenschaftlichen Weiterbildung als Resultante eines vierfachen Zielgruppenbezugs. In: Pätzold, H./ Felden, H. von/Schmidt-Lauff, S. (Hrsg.): Programme, Themen und Inhalte der Erwachsenenbildung. Hohengehren: Schneider, S. 154-169. 
Seitter, W. (2014): Nachfrageorientierung als neuer Steuerungsmodus. Wissenschaftliche Weiterbildung als organisationale Herausforderung universitärer Studienangebotsentwicklung. In: Weber, S.M./Göhlich, M./Schröer, A./Schwarz, J. (Hrsg.): Organisation und das Neue. Beiträge der Kommission Organisationspädagogik. Wiesbaden: Springer VS, S. 141-150.

Seitter, W./Schemmann, M./Vossebein, U. (2015): Bedarf - Potential - Akzeptanz. Integrierende Zusammenschau. In. Dies (Hrsg.): Zielgruppen in der wissenschaftlichen Weiterbildung. Empirische Studien zu Bedarf, Potential und Akzeptanz. Wiesbaden: Springer VS, S. 23-59.

Walber, M./Jütte, W. (2015): Entwicklung professioneller Kompetenz durch didaktische Relationierung in der wissenschaftlichen Weiterbildung. In: Hartung, O./Rumpf, M. (Hrsg.): Lehrkompetenzen in der wissenschaftlichen Weiterbildung. Konzepte, Forschungsansätze und Anwendungen. Wiesbaden: Springer VS, S. 49-64.

Weber, K. (2010): Aushandlung von Zeitregimes in der Weiterbildung. In: Schönbächler, M.-T./Becker, R./Hollenstein, A./Osterwalder, F. (Hrsg.): Die Zeit der Pädagogik. Zeitperspektiven im erziehungswissenschaftlichen Diskurs. Bern: Haupt Verlag, S. 103-118.

Wolter, A./Banscherus, U. (2016): Lebenslanges Lernen und akademische Bildung als internationales Reformprojekt. In: Wolter, A./Banscherus, U./Kamm, C. (Hrsg.): Zielgruppen Lebenslangen Lernens an Hochschulen. Münster-New York: Waxmann, S. 53-80.

Wolter, A./Geffers, J. (2013): Zielgruppen lebenslangen Lernens an Hochschulen - Ausgewählte empirische Befunde. Berlin-Oldenburg.

Wolter, A./Banscherus, U./Kamm, C. (2016) (Hrsg.): Zielgruppen Lebenslangen Lernens an Hochschulen. Münster-New York: Waxmann. 



\section{Wissenschaftliche Weiterbildung im Medium von Praxisforschung - eine relationstheoretische Deutung}

ORTFRIED SCHÄFFTER

\section{Einführung}

Obwohl wissenschaftliche Weiterbildung dem Traditionsbestand von Universitäten und wissenschaftlichen Hochschulen zugerechnet wird, ist sie bis heute noch nicht institutionell im Kernbereich des Wissenschaftssystems verankert, sondern stellt als „Universitäre Erwachsenenbildung“ eine strukturell angelagerte Erweiterung ihrer Lehrfunktion dar, auf deren Ausweitung in „extra-murale“ Bereiche im Notfall auch verzichtet werden könnte. Dieser prekäre Status wissenschaftlicher Weiterbildung im Gefüge des Wissenschaftsbetriebes manifestiert sich in immer wieder aufflackernden Bestandskrisen und einer Tendenz zu ihrem „outsourcing“ in einen sich kommerzialisierenden Weiterbildungsmarkt. Offenbar bekommt man es weiterhin mit Legitimationsproblemen zu tun. Dies steht in auffälligem Gegensatz zur bildungspolitischen Programmatik einer öffentlichen Dienstleistungsfunktion von Wissenschaft, aber auch zu ihrer rechtlichen Verankerung in den Hochschulgesetzen.

In den folgenden Überlegungen wird die widersprüchliche Lage wissenschaftlicher Weiterbildung auf ihre institutionelle Randständigkeit zurückgeführt, die sich allerdings nicht aus pragmatisch zu behebenden Mängeln ihrer pädagogischen Organisation erklären lässt. In diesem Fall hätte sich die prekäre Lage schon längst durch Optimierung ihrer Einrichtungsorganisation überwinden lassen und damit durch all die Bemühungen, wie sie in den letzten Jahrzehnten zu keinen strukturell nachhaltigen Auswirkungen geführt haben. So kommt man nicht an der Einschätzung vorbei, dass die Problematik tiefer angelegt ist. Sie geht in erheblichem Maße auf ein noch immer traditionell geprägtes Verhältnis zwischen Wissenschaft und Gesellschaft zurück. Pointiert formuliert heißt dies: Die periphere Lage und der unsichere Status wissenschaftlicher Weiterbildung in den Institutionalformen des Wissenschaftssystems erweisen sich als symptomatisches Folgeproblem einer mittlerweile obsoleten Engführung auf ein dis- 
ziplinär eingehegtes Wissenschaftsverständnis, das keine unmittelbare "Resonanz" (Rosa 2016) in gesellschaftlichen Umwelten auszulösen vermag.

Aus dieser Diagnose wird auch die Stoßrichtung möglicher Strategien zur Problemlösung ableitbar: Eine zukunftsweisende Entwicklung von nachhaltigen Formaten wissenschaftlicher Weiterbildung muss ihre Dynamik aus einer „Transformativen Wissenschaft" (Schneidewind/Singer-Brodowski 2014) beziehen. Dies verlangt, wie im Titel angekündigt, eine „relationstheoretische Sicht“ (Schäffter 2012; 2014) auf Wissenschaft. Eine gesellschaftlich wirkungsvolle Institutionalisierung wissenschaftlicher Weiterbildung setzt voraus, dass man „Wissenschaft neu denken“ (Nowotny/ Scott/Gibbons 2014) kann, und das verlangt zugleich, das „Verhältnis der Wissenschaft zu Politik, Wirtschaft und Medien in der Wissensgesellschaft" (Weingart 2005; 2015) in seiner gegenwärtigen Transformation genauer $\mathrm{zu}$ verstehen. Der Strukturwandel im Verhältnis von „Wissen und Öffentlichkeit in einem Zeitalter der Ungewissheit“ (Weingart 2005) ist bereits voll im Gange. Wissenschaftliche Weiterbildung lässt sich in diesem Zusammenhang als strukturelle Schnittstelle zwischen dem Wissenschaftssystem und seiner gesellschaftlichen Umwelt fassen, aus der heraus sich in relevanten Praxisfeldern ein für das Funktionssystem resonantes „Publikum“ (Burzan et al. 2008) herauszubilden und auf Dauer zu stellen vermag. Unter der soziologischen Kategorie „Publikum“ wird somit der komplementäre Resonanzboden für die Leistung eines Funktionssystems in ihrer gesellschaftlichen Umwelt verstanden. Das sich gegenwärtig wandelnde Verhältnis (Nowotny 1999) stellt hierbei für die erkenntnistheoretische Grundlegung wissenschaftlicher Weiterbildung

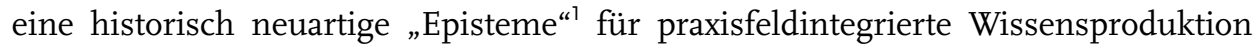
dar. Es entwickeln sich über den bislang produktförmigen Wissenschaftstransfer hinaus Bildungsformate zur Generierung von wissenschaftlich reflektiertem Praxiswissen. Das ist eine noch ungewohnte Sichtweise. Während das Wissenschaftssystem traditionell in seinem funktionsbezogenen Leistungsaspekt selbstreferenziell auf ihren Innenraum bezogen blieb (Stichweh 1988; Burzan et al. 2008, 31) und daher komplementär zu ihrer Erkenntnisleistung und Theoriebildung keine komplementäre „Publikumsrolle“ in gesellschaftlichen Umwelten auszubilden hatte (Hellmann 2010, 26), schlägt nun ihr Verhältnis zur gesellschaftlichen und politischen Umwelt in eine bislang noch wenig verstandene Koproduktion praxisfeldnaher Wissensgenerierung um. Einrichtungen wissenschaftlicher Weiterbildung erhalten mit dem Herausbilden von Publikumsrollen des Wissenschaftssystems nun die strukturelle Voraussetzung, zwischen Wissenschaft und Praxis lernförmige Forschungsleistungen für ausgewählte Adressatenbereiche in Praxisfeldern zu organisieren. Sie übernehmen im Rahmen von Praxisforschung eine reziproke Scharnierfunktion zwischen disziplinärer Wissensproduktion und praxisbasierter Wissensverwendung. In ihrer intermediären Lage zwischen Wissenschaft und gesellschaftlichen Verwendungssituationen werden sie zum Mitakteur eines strukturellen Wandels, in dem

1 Vgl. zum Wandel epistemischer Hintergrundannahmen in der Wissenschaftsgeschichte Rheinberger 2007 und zur „gestalterischen Diagrammatik“ Dell 2016. 
sich die gesellschaftliche Funktion von Wissenschaft grundlegend zu transformieren beginnt. Wissenschaftliche Weiterbildung wird dabei nolens volens zu einer institutionellen Mitspielerin innerhalb einer hochschulpolitischen Arena, in der sie die Transformation von Forschung unter einer bildungstheoretischen Perspektive unterstützend voranzutreiben vermag. Voraussetzung ist allerdings, dass sich wissenschaftliche Weiterbildung ihres eigenen "Gegenstandes" auf einer kategorialen Grundlage zu vergewissern vermag, d.h. dass sie sich tatsächlich als genuin „wissenschaftliche" Weiterbildung versteht und dadurch in ihrem Funktionsbezug von den Institutionalformen des Bildungssystems dezidiert zu unterscheiden weiß. Dies ist bisher nur unzureichend der Fall und gehört damit zur eigentlichen Ursache ihrer Randständigkeit im Wissenschaftsbetrieb. Eine Lösung wäre daher in der Klärung zu suchen, wie Weiterbildung nicht allein zu einem produktförmig zugeschnittenen "Wissenschaftstransfer" beiträgt, sondern auch zum Medium einer praxisfeldintegrierten Generierung von transdisziplinärer Theorie werden könnte. Der transformative Strukturwandel läuft somit auf „Bildung im Medium von Forschung“ und dies in Verbindung von "Praxisforschung im Medium von Weiterbildung" hinaus (vgl. Schäffter 2017). Im Zusammenhang eines "translational turn“ (BachmannMedick 2006, S. 238; Renn 2002) von wissenschaftlicher Weiterbildung könnte sie eine intermediäre Übersetzungsfunktion übernehmen und den Bildungswissenschaften aufgrund ihrer Transdisziplinarität zukünftig die Rolle einer paradigmatischen Musterlösung im Rahmen eines anstehenden paradigmatischen Wandels zukommen.

In der folgenden Argumentation geht es folglich darum, eine solche Entwicklungsperspektive schrittweise im Einzelnen zu begründen, sie mit dem aktuellen Diskurs zur Transformation von Wissenschaft in Beziehung zu setzen, um am Ende zu hochschulpolitischen Entwicklungsperspektiven für wissenschaftliche Weiterbildung in den Universitäten und Hochschulen zu gelangen.

\section{Bisherige Grenzen einer Institutionalisierung von wissenschaftlicher Weiterbildung}

\subsection{Zum bisherigen Selbstverständnis wissenschaftlicher Weiterbildung}

Das besondere Qualitätsmerkmal von „wissenschaftlicher“ Weiterbildung ist im Konkurrenzverhältnis zu anderen Anbietern von Erwachsenenbildung keineswegs geklärt, geschweige konsensfähig. Schließlich kann im Kontext einer modernen Wissensgesellschaft und im Rahmen der Akademisierung und Professionalität des Weiterbildungspersonals jedes seriöse Bildungsangebot für sich "Wissenschaftlichkeit" in Anspruch nehmen, solange es sich auf theoretische Grundlagen und Forschungsergebnisse einer Referenzdisziplin beruft. Wissenschaftliche Weiterbildung definiert sich im Spektrum unterschiedlicher Profile daher keinesfalls aus dem Gegensatz $\mathrm{zu}$ „unwissenschaftlichen“ Bildungsangeboten. Ihr Alleinstellungsmerkmal bezieht 
sie vielmehr aus einem institutionellen Kontext. Das besondere Leistungsprofil beruht auf einer organisatorischen Anbindung der Trägereinrichtung an das Wissenschaftssystem und dessen Institutionalformen „Universität“ und „Hochschule“. Das Label „Wissenschaftlichkeit“ verweist somit weniger auf eine inhaltlich höherwertige Qualität ihrer Angebote, sondern auf ihre institutionelle Einbettung in den wissenschaftszentrierten Lehrbetrieb von Universität und Hochschule mit ihren je besonderen hochschuldidaktischen Lehrformen und formalisierten Abschlussmöglichkeiten. Im Zusammenhang von öffentlichen Vorlesungen, Seminarkursen und weiterbildenden Studiengängen für Adressaten jenseits des tertiären Sektors berufsqualifizierender Bildung handelt es sich um tradierte Formen einer „Universitätsausdehnung “ und damit um eine zusätzliche Ausdifferenzierung disziplinärer oder interdisziplinärer Lehrangebote für immer umfassendere Adressatenbereiche im regionalen und fachlichen Einzugsbereich einer Hochschule. Für sie werden Lehrangebote einer „berufsbefähigenden“ wissenschaftlichen Bildung ${ }^{2}$ im Sinne einer zielgruppenorientierten Didaktik weiteren Personengruppen oder Bildungsmilieus zugänglich gemacht. Das bildungspolitische Leitziel, die wissenschaftlichen Ressourcen, die sich in einer Hochschule als einer öffentlichen Ressource kumuliert vorfinden, für mögliche Nutzergruppen in geeigneter Weise zu erschließen, verweist darauf, dass einem anbieterzentrierten Planungsmodell gefolgt wird. Die Wissensproduktion in der disziplinären Forschung selbst folgt zunächst einer weitgehend anderen Logik als ihre in der Lehre nachfolgende pädagogische Distribution in jeweils thematisch und methodisch passend erscheinende Felder möglicher „Anwendung“. Die spezifische Stärke von Angeboten wissenschaftlicher Weiterbildung folglich auf einer Öffnung der universitären Lehre, die zwar hochschuldidaktisch für eine nicht-studentische Hörerschaft aufbereitet wird, aber dennoch den je besonderen Qualitätsmerkmalen und inhaltlichen Studienschwerpunkten der anbietenden Hochschule und den disziplinären Lehrkompetenzen ihres wissenschaftlichen Personals verpflichtet bleibt. Als ihre strukturelle Schwäche kann gesehen werden, dass sie aufgrund ihrer Einbindung in eine disziplinäre Fachlichkeit von einer vorgegebenen Fachsystematik ausgeht und daher notwendigerweise dem Modus einer „applied science“, also der Anwendung disziplinär zugerichteter Wissensbestände verpflichtet bleibt, deren Quelle der Wissenserzeugung dezidiert in einer „relationalen Distanz“ (vgl. Ibert/Müller/Stein. 2014, 2. Kap.) zur lebensweltlichen Alltagspraxis steht und deren wissenschaftlichen Erkenntnisse in Abgrenzung zu ihr generiert werden. Aus diesem Strukturbruch folgt als „pädagogisch“ zu lösendes Problem, dass nun ein „Transfer“ in einen als „außerwissenschaftlich“ definierten Praxiskontext hochschuldidaktisch zu bewerkstelligen ist. Ähnlich wie bei der innerwissenschaftlichen Ausbildung führt diese Variante disziplinärer Anbieterzentrierung in der Regel dazu, dass ein fachsystematischer Fokus schon aufgrund der Studien- und Prüfungsordnungen Priorität erhalten muss, sodass die möglichen Transferleistungen nur se-

2 Vgl. zur „Wissenschaftlichkeit" einer Berufsausbildung: Fuer-eine-demokratische-und-soziale-Hochschule-hochschulpolitisches-Programm-des-DGB.pdf http://www.dgb.de/themen/++co++61926a20-4053-11e2-a408-00188b4dc422 
kundäre Bedeutung haben und oft genug der weiteren Praxissozialisation und damit den individuellen Lernbiografien überlassen bleiben.

Vor dem Hintergrund der hier skizzierten disziplinären Anbieterzentrierung von wissenschaftlicher Weiterbildung erscheint es als zweifelhaft, dass eine Öffnung universitärer Lehrveranstaltungen für Berufspraktikerinnen und -praktiker zu innovativen Rückwirkungen innerhalb der Hochschule im Sinne größerer Praxisnähe der Hochschuldidaktik führen könnte. Obwohl derartig belebende Impulse in die disziplinäre Lehre gern zur Legitimation von wissenschaftlicher Weiterbildung angeführt werden, erweist sich eine derartige Wechselwirkung im derzeit gegebenen Wissenschaftsverständnis letztlich als „frommer Wunsch“.

\subsection{Weiterbildung, ein verzichtbarer Appendix im Wissenschaftsbetrieb?}

In institutionstheoretischer Deutung folgen die bisher praktizierten Formate wissenschaftlicher Weiterbildung dem Typus einer „symbiotischen“ Anlagerung ihrer pädagogischen Dienstleistungen an das Wissenschaftssystem und seiner Funktionslogik.

Ansätze wissenschaftlicher Weiterbildung folgen in der Mehrzahl einem „symbiotischen Schnittstellentypus“. Sie fungieren als strukturelle Schnittstelle zwischen dem Anlehnungssystem Wissenschaft und dem Anwendungssystem Bildung und setzen somit die disziplinäre Logik ihres bedeutungsverleihenden "Anlehnungssystems“ als faktische Gegebenheit voraus. In dieser funktionalen Anlehnung bieten sie pädagogische Dienstleistungen, mit denen die gesellschaftliche Wirksamkeit des „Funktionssystems Wissenschaft“ im Aufgabenbereich Lehre mit pädagogischen Mitteln unterstützt wird. Analoge Strukturbedingungen finden sich in symbiotischen Institutionaltypen wie Pflegepädagogik, Sozialpädagogik, Gesundheitsbildung oder innerbetriebliche Weiterbildung. „Symbiotische Anlagerung“ von Bildung an ein Funktionssystem setzt jedoch voraus, dass sie dem Anlagerungssystem aufgrund der Instrumentalität des Pädagogischen „äußerlich“ bleibt und somit im Falle des Wissenschaftssystems keinerlei Zugang zu dessen Kernkompetenzen der Erzeugung wissenschaftlichen Wissens erhält. In letzter Konsequenz bedeutet dies, dass ein Bildungsformat wissenschafticher Weiterbildung keinerlei relevante Auswirkung auf die Konzeptualisierung und praktische Durchführung von Forschung haben kann. „Lehre" hat im gegenwärtig noch substanzialistisch gefassten Verständnis von Wissenserzeugung keinerlei Einfluss auf die Forschungspraxis. Disziplinäre Forschung liegt wissenschaftlicher Weiterbildung ähnlich wie auch der hochschuldidaktisch gestalteten Lehre konstitutiv voraus und begründet damit ihr wissenschaftstheoretisches Ableitungsverhältnis im herkömmlichen Wissenschaftsbetrieb. Die damit beschriebene Trennung ist keine neue Erkenntnis und wird seit Wilhelm von Humboldts Programmatik einer "forschenden Lehre“ zumindest im deutschsprachigen Raum kontrovers diskutiert als ein wissenschaftstheoretisch klärungsbedürftiges Verhältnis zwischen der Praxis forschenden Lernens und bildender Umweltaneignung. Fraglos bewegt sich wissenschaftliche Weiterbildung weiterhin innerhalb dieser epistemologischen Spannungslage. 
Solange also pädagogische Dienstleistung im Wissenschaftssystem weitgehend instrumentell auf den Transfer von disziplinär erzeugten Wissensbeständen verstanden wird, kann das konstitutive Spannungsverhältnis zwischen der Weiterbildungspraxis und einer das wissenschaftliche Wissen in den Verwendungssituationen generierenden Forschungspraxis gar nicht erst auf der Bildfläche erscheinen.

Mit dieser Problembeschreibung ist allerdings bereits ein wichtiger Schritt getan. Man wird angesichts des oben erwähnten internationalen Diskurses zu einer „transformativen Wissenschaft“ im „mode 2“ partizipatorischer Wissenserzeugung oder im Rahmen einer „aufgabenorientierten Wissenschaft“ (Ostermeyer/Krüger 2015) darauf aufmerksam, dass die Inkompatibilität von Forschung und Bildung möglicherweise auf ein mittlerweile obsolet gewordenes Verständnis von wissenschaftlicher Forschung und damit auch von Bildung zurückzuführen sein könnte. Hiermit ist der Punkt erreicht, an dem sich die Frage nach einem Wissenschaftsverständnis stellt, in dem das Verhältnis von Bildung und Forschung nicht mehr substanzialistisch als Wissenstransfer gefasst, sondern relationstheoretisch als produktive Wechselwirkung zu gesellschaftlichen Praxiskontexten reformuliert werden kann. Worin aber besteht dazu eine ernst $\mathrm{zu}$ nehmende Alternative? Bei der bereits oben eingeführten Forderung, Wissenschaft in ihrem Verhältnis zu der sie konstituierenden Gesellschaft „neu zu denken“ (Nowotny/Scott/Gibbons 2014), kreist der Diskurs um Suchbegriffe wie „Postnormal Science“ (Funtowicz/Ravetz 2010), „Postacademic Science“ (Ziman 1996) oder auch „Aufgabenorientierte Wissenschaft“ (Ostermeyer/ Krüger 2015), wobei das ihnen gemeinsame Anliegen auf eine „praxissensible Forschung“ (Wolff 2008) bzw. eine „Context-Sensitive Science“ (Gibbons 2000) hinauszulaufen scheint. Ganz offensichtlich geht es um eine sehr grundsätzliche Frage, wie Wissenschaft und Gesellschaft bereits in der Forschungspraxis zueinander ins Verhältnis gesetzt werden können (vgl. als paradigmatisches Musterbeispiel Dell 2016). Um diesen Klärungsschritt vorzubereiten, scheint es hilfreich, eine relationstheoretische Sicht auf Wissenschaft und Forschung einzuführen.

\section{Wissenschaft und Forschung aus relationstheoretischer Sicht}

Bei den wissenschaftshistorischen Hintergrundannahmen einer „Transformationsgesellschaft“ (Schäffter 2001) gehen wir von einer langfristigen epistemischen Transformationsbewegung aus, der zufolge sich schon seit Längerem die Gegenstandsbestimmung in nahezu allen Wissenschaftsdisziplinen von einer substanziellen Konzeptualisierung löst und sich dabei tendenziell einer relationalen Formation annähert. Dies gilt nicht allein für die Naturwissenschaften, sondern immer deutlicher auch für die Sozial- und Kulturwissenschaften. Ein derartig übergreifender Paradigmenwechsel ließe sich als „relational turn“ auf einer transdisziplinären Ebene im Sinne eines wissenschaftshistorischen Megatrends formulieren. Er ist zudem an 
Untersuchungen einer „historischen Epistemologie“ (Rheinberger) bereits in einschlägigen Diskursverläufen verschiedener Einzelwissenschaften $\mathrm{zu}$ belegen. Im Forschungskontext einer „historischen Epistemologie“ wird die Entwicklung von einer zunächst substanzialistisch gefassten Gegenstandsbestimmung hin zu einem relationstheoretisch gefassten „epistemischen Objekt“ erkennbar. Was aber ist unter einer relationstheoretischen Rekonzeptualisierung eines Forschungsgegenstandes $\mathrm{zu}$ verstehen?

\subsection{Relationalität - eine erste Begriffsklärung}

Begriffshistorisch lassen sich zwei grundlagentheoretisch konträre Positionen bei der Bestimmung von Relationalität unterscheiden: nämlich zwischen einer substanzialistischen und einer relationalen. Diese Zugänge führen zu der Differenz einer „externen“ und einer „internen“ Relation.

- Bei einer externen, substanzialistisch gefassten Sicht geht man von vorfindlichen, einer Relation bereits vorgegebenen Elementen aus, die zueinander ins Verhältnis gesetzt werden und hierdurch erst nachträglich zu Relata werden. Die „Dinge“ gehen aus dieser Sicht in ihrem „Selbststand“ der Relation voraus.

- Bei einer konsequent „nicht-essenzialistischen“, also relationalen Sicht konstituieren sich die dinghaften Relata erst aus ihrem jeweiligen Beziehungsverhältnis heraus. Die Relation geht aus dieser Sicht den „Dingen“ voraus. Dies kommt vor allem in Netzwerkkonzepten oder ähnlich korrelativ gefassten Beziehungsstrukturen (bspw. Sprache) zum Ausdruck. ${ }^{3}$

\subsection{Eine substanzialistische und eine relationstheoretische Grundlegung von Forschung und Bildung}

Im Alltagsverständnis geht man von getrennten, objekthaften Gegenständen aus, die zunächst als eigenständig betrachtet und erst sekundär mit anderen ebenfalls eigenständigen Objekten in Beziehung gebracht werden. Metaphorisch ließe sich eine solche Gegenstandsbetrachtung als „Billard-Modell“ bezeichnen: hier „stoßen sich die Dinge hart im Raume“. Anders verfahren wir bei „relationalen“ Begriffen: kein Zwilling ohne den anderen, keine Mutter ohne ihr Kind, keine Fremdheit ohne die Eigenheit, kein System ohne seine systemspezifische Umwelt, keine Vorderseite der Münze ohne ihre Rückseite. Niklas Luhmann fasst in Anschluss an Spencer Brown Relationalität als „Logik der Form“. Kurz gesagt, es geht um „dialektisches Denken“. Form ist beidseitig und beruht auf „Zweiseitigkeit“. In einer relationstheoretischen Gegenstandsbestimmung geht es folglich darum, die im alltäglichen Zusammenhang getrennt wahrgenommenen Objekte daraufhin $\mathrm{zu}$ befragen, ob sie nicht als relationale Begriffe weitaus zutreffender verstanden werden als in der Unterstellung ihrer substanziell getrennten Eigenständigkeit. Ist beispielsweise „Behinderung“ eine besondere objektive Eigenschaft, die ich einem anderen zuschreiben kann, oder

3 In Anlehnung an von Wolzogen 1992. 
gehören dazu vielmehr nicht mindestens zwei? Nämlich einer, der jemanden behindert, und ein anderer, der behindert wird. „Behinderung“ lässt sich somit zutreffenderweise begrifflich intransitiv als substanziell zuschreibbare Eigenschaft und auch transitiv als Beziehungsmodus benutzen. Genau auf diese Unterscheidung bezieht sich der Übergang von einer substanzialistischen hin zu einer relationstheoretischen Konzeptualisierung eines Forschungsgegenstandes. An dem Beispiel der Einseitigkeit einer Zuschreibung von Behinderung wird erkennbar, dass der beabsichtigte Perspektivwechsel fort von einer substanziellen und hin zur Beidseitigkeit einer relationalen Sicht tief greifende Veränderungen in der Gegenstandsbestimmung auslöst, die nicht nur erkenntnistheoretische, sondern zutiefst politische Folgen haben kann und vielfach auch soll. Im Rahmen der kategorial angesiedelten Rekonstruktion eines Begriffs werden nämlich neuartige und bisher noch unbekannte „Hinsichten" auf den sich hierbei klärenden Forschungsgegenstand erschlossen, der damit in einer relationslogischen Hinsicht erst intelligibel, das heißt nun überhaupt erst empirisch erkennbar wird. Den Übergang von einer substanziellen hin zu einer relationslogischen Hinsicht bezeichnen wir als "relationstheoretische Rekonzeptualisierung" eines Gegenstandes. Sie setzt zunächst die Einnahme einer Kontingenzperspektive auf den Gegenstand voraus und beruht damit auf der Erfahrung eines Scheiterns des bisher gültigen kategorialen Deutungshorizonts. Man muss also erst schmerzlich erkennen, dass "das Fremde“ an einem mir begegnenden Menschen keine "Eigenart" ist, die er zu seiner Integration tunlichst „ablegen“ sollte, sondern dass es sich um einen Beziehungsmodus handelt, an dem auch meine persönliche „Eigenart" konstitutiv beteiligt ist.

Um zwischen einer substanzialistischen zu einer relationstheoretischen „Hinsicht“ wechseln zu können, bedarf es zunächst einer „Kontingenzperspektive“ auf ein bislang unhinterfragt als gegebenes Faktum hingenommenes Phänomen (Schäffter 2011; 2012). Dieses Hinterfragen meint man sich vor allem in einer empiristischen Forschungshaltung ersparen zu können, um sich inhaltlich gehaltvolleren Fragen widmen zu können. Dies birgt allerdings die Gefahr, auf ein naives Alltagsverständnis zurückzufallen. Der Frankfurter Relationsphilosoph Julius Schaaf fasste die Problematik folgendermaßen zusammen: „Da aber die Fachwissenschaften diese ihre Voraussetzungen selbst nicht mehr wissenschaftlich untersuchen, sondern einfachhin unterstellen, kann es sich durchaus ereignen, daß sie, von ihnen unbemerkt und unbewusst, von fehlerhaften Prämissen ausgehen, die sich dann in der Anwendung und d.h. in der Praxis unheilvoll auswirken können. Da nun seit dem Beginn der Neuzeit die Fachwissenschaften in steigendem Maße das Gebiet der Praxis eroberten, so konnte es nicht ausbleiben, daß Irrtümer im Grundlegenden verhängnisvolle Auswirkungen haben mussten. Da es nun Aufgabe der Philosophie ist, eben diese Voraussetzungen kritisch $\mathrm{zu}$ prüfen und wenn nötig zu korrigieren, so ergibt sich hieraus der zunächst unerwartete Sachverhalt, daß auch die anscheinend rein 
theoretische Philosophie eine bedeutsame praktische Relevanz gewinnen kann." (Schaaf) ${ }^{4}$

Im Rahmen einer Methodologie kategorial reflektierter Bestimmung bzw. Konstitution des Forschungsgegenstandes gerät die kritische Auseinandersetzung mit den epistemologischen Hintergrundannahmen in den Fokus einer nun transdisziplinär angelegten Forschung. Die Auseinandersetzung mit der Trennung zwischen Philosophie und Einzeldisziplin lässt sich auf das ungeklärte Spannungsverhältnis bei der bildungswissenschaftlichen Gegenstandsbestimmung übertragen.

Von Interesse für eine Methodologie relationstheoretisch angelegter Weiterbildungsforschung ist hierbei eine kritische Distanznahme zu den disziplinär latent einsozialisierten epistemologischen „Erblasten“.

Der inzwischen historisch überfällige Wechsel von einer substanziell gefassten Gegenstandsbestimmung von wissenschaftlicher Weiterbildung im Sinne eines Transfers von disziplinär generierten Kenntnissen und empirisch belegbaren Fakten hin $\mathrm{zu}$ kontextgebundenem Wissen aus der reflektierten Binnensicht eines praktischen Verwendungszusammenhangs verlangt in einem ersten Schritt zunächst, dass eine Kontingenzperspektive auf den Forschungsgegenstand eingenommen werden kann. Eine relationstheoretische Rekonzeptualisierung bislang substanziell gefasster Forschungsgegenstände erfolgt in einem nächsten Schritt dadurch, dass dieser als ein Beziehungsproblem gefasst wird. Im hier diskutierten Zusammenhang zielt eine relationstheoretische Rekonzeptualisierung auf das Beziehungsfeld zwischen einer disziplinären und einer praxisfeldbezogenen Gegenstandsbestimmung ab. Je nach Standort der Wissensproduktion bekommt man es mit einer besonderen Perspektive auf den Forschungsgegenstand $\mathrm{zu}$ tun. Entscheidend für einen relationstheoretischen Deutungszusammenhang ist nun, keine der beiden Konstitutionsbedingungen zu verabsolutieren, sondern sie korrelativ in ihrer „relationalen Distanz" im Sinne einer „produktiven Differenz" (Ibert/Müller/Stein 2014) zueinander ins Verhältnis zu setzen. Dies erfolgt nicht in der Vollzugslogik eines linearen Transfers, sondern als dialogischer Aushandlungsprozess, wie er von Gibbons u.a. als Lernprozess im „mode 2“ beschrieben wurde. An diesem Punkt wird die strukturelle Transformation erkennbar, die in diesem Kontext wissenschaftliche Weiterbildung erfahren muss. Sie wird zum Medium einer Translation ${ }^{5}$ zwischen zwei nicht kompatiblen, aber komplementär aufeinander bezogenen Sichtweisen auf einen Forschungsgegenstand, der weder allein disziplinär noch aus einer Praxis heraus konstituiert wird. Aus ihrer Nähe und Distanz eröffnet sich ein Beziehungsraum zur gemeinsamen Generierung von Wissen (vgl. auch Reichwald/Piller 2006). Was darunter genauer zu verstehen ist, soll

4 Vgl. auch Schaaf 1966; Schäffter 2012, 2014.

5 Zur „interaktiven Wertschöpfung“ im Rahmen eines translatorischen Paradigmas vgl.: Bachmann-Medick 1997; Renn 2002; Böhmer 2014; Reichwald/Piller 2006; Bonnemeier/Reichwald 2012; Ibert/Müller/Stein 2014. 
an der nachfolgenden Unterscheidung zwischen zwei Quellen der Erzeugung wissenschaftlichen Wissens verdeutlicht werden.

\subsection{Zwei Weisen der Erzeugung von wissenschaftlichem Wissen}

Die klassische Dichotomie zwischen einer substanzialistischen und einer beziehungsbasierten Gegenstandsbestimmung scheint inzwischen mit dem wissenssoziologischen Erklärungsansatz von Gibbons et al. (1994) zugunsten einer korrelativen Auffassung überwunden worden zu sein (vgl. auch Nowotny 1999; Gibbons 2000; Bender 2001; Nowotny Scott/Gibbons 2001). Für die Konzeptualisierung von wissenschaftlicher Weiterbildung im Medium von Praxisforschung ermöglicht ihr Ansatz eine beidseitig sowohl von disziplinärer Theorie als auch praxisfeldbasierter Expertise getragene „Produktion von Wissen“, sodass nicht mehr eine der beiden Positionen die andere dominiert, sondern sich beide Seiten wechselseitig aussteuern. Wie der Titel ihrer Schrift „The New Production of Knowledge” bereits signalisiert, geht es dem internationalen Autorenteam um Gibbons und Nowotny nicht mehr allein um einen auf „Anwendung“ gerichteten Transfer wissenschaftlichen Wissens im Sinne disziplinär bereitgestellter Wissensbestände, also um „Wissensdistribution“ oder „Wissensmanagement“, sondern um soziale Kontexte der Wissensproduktion, in denen zwei differente Paradigmen in einer „community of practice“ produktiv zueinander ins Verhältnis gesetzt werden. Die Formen gesellschaftlicher Wissensproduktion unterliegen zudem einem historischen Wandel (Meier-Rabler 1990). Traditionelle Kontexte disziplinär fundierter Wissensproduktion an Universitäten und Forschungseinrichtungen werden zunehmend durch extramurale „contexts of knowledge production“ erweitert. Mit ihnen werden auch Schnittstellen zwischen unterschiedlichen Wissensordnungen (Ibert/Müller/Stein 2014) als differente Kontexte der Wissensproduktion bestimmbar und empirisch beobachtbar. Diese Kontexte lassen sich als praxisfeldgebundene „Lernwelten“ fassen, die allerdings nicht mit dem Konzept eines unreflektierten „Alltagswissens“ latenter Routinestrukturen zu verwechseln sind. Konsequent unterschieden wird daher zwischen zwei differenten Quellen der Wissensproduktion, die nun in „relationaler Distanz“ in translatorischen „Beziehungsräumen der Wissensgenerierung“ (Ibert/Müller/Stein 2014) füreinander fruchtbar gemacht werden, weil mit ihnen zwei komplementäre Konstitutionsbedingungen eines Forschungsgegenstandes in einem dialogisch angelegten Aushandlungsprozess korrelativ aufeinander bezogen werden können.

\section{Mode 1: Disziplinäres Wissen und seine Anwendung}

Traditionell wird von einer disziplinär verankerten Erzeugung von Wissen im Kontext von fachlichen Forschungsdesiderata und ihnen gemäßen Problemfassungen ausgegangen. Wissenschaftliche Forschungsergebnisse werden aus der tradierten Systematik eines historisch entstandenen Wissenschaftssystems hervorgebracht; und so steht bei ihnen die Anwendbarkeit auf außerwissenschaftliche Problemlösungen zunächst nicht im Vordergrund, wenn diese auch nicht ausgeschlossen wird. Die Erzeugung disziplinärer Wissensbestände zielt folglich primär auf Er- 
kenntnisgewinn im Rahmen systematisch aneinander anschließender Forschungserträge. So findet sich hier auch ein Verständnis in Bezug auf das Verhältnis von Theorie und Praxis, das von einem Primat der disziplinären Wissensproduktion ausgeht und dabei deutliche Abgrenzungen gegenüber „angewandter“ Forschung und einer aus disziplinärem Wissen sekundär abgeleiteten „angewandten Wissenschaft“ zieht. In diesem Verständnishorizont von struktureller „Translation“ lassen sich die Diskurse um "Wissenschaftstransfer" oder zu einer Transformation zunächst noch „reiner" disziplinärer Wissensbestände im degenerierend vermischenden Prozess ihrer praktischen Anwendung verorten (Dewe 1988; Wingens/Weymann1991; Beck/ Bonß 1989). Auch die disziplinären Beziehungen zwischen sogenannten „Handlungswissenschaften“ wie z. B. Pädagogik zu ihren grundlagentheoretisch angelegten Referenzdisziplinen Psychologie, Soziologie und Geschichte werden noch immer unter einem derartigen disziplinär eingehegten Theorie-Praxis-Verhältnis diskutiert. Dies ist der essenzialistische Bedeutungshorizont, in dem die gegenwärtige Praxis wissenschaftlicher Weiterbildung noch disziplinär eingehegt wird.

\section{Mode 2: Erzeugung von Wissen im transdisziplinären Kontext}

Wissensproduktion erfolgt hier in praktischen Verwendungszusammenhängen. Gibbons et al. (1994) heben in diesem Zusammenhang hervor, dass eine derartige wissenschaftliche Forschung in der späten Moderne dabei ist, aus den tradierten Institutionalformen des Wissenschaftssystems auszuwandern, sodass Forschungsinstitute und Universitäten durch verwendungsbasierte Kontexte der Wissensproduktion in lebensweltlich verankerter Alltagspraxis deutliche Konkurrenz erfahren und somit gerade dabei sind, ihr bisheriges Alleinstellungsmerkmal als Quelle wissenschaftlicher Wissensbestände zu verlieren. Dies wirft ein völlig neues Licht auf praxisfeldintegrierte Verfahren der Theoriegenerierung, mit denen man in entwicklungsbegleitender Weiterbildung bereits wichtige Erfahrungen sammeln konnte, deren Ergebnisse jedoch nur schwer in disziplinäres Wissen integrierbar waren und im Rahmen von Evaluation vorwiegend aus dieser Engführung heraus beurteilt wurden. Offenbar bekommt man es hier mit einem neuen Typus einer "Production of Knowledge“ zu tun, der bisher noch nicht wissenschaftstheoretisch einzuordnen war, solange man ausschließlich disziplinzentriert zu denken pflegte. Derartige praxisfeldimmanente Formen der Erzeugung von wissenschaftlichem Wissen bezeichnet die Autorengruppe um Gibbons im Kontrast zur disziplinären Wissensproduktion im Sinne eines Arbeitsbegriffs als „mode 2“:

"The transformation is described in terms of the emergence alongside traditional modes of knowledge production that we will call Mode 2. By contrast with traditional knowledge, which we call Mode 1, generated within a disciplinary, primarily cognitive context, Mode 2 knowledge is created in broader, transdisciplinary social and economic contexts." (Gibbons et al. 1994, S. 1) 
Beim Konzept einer aus dem Praxisfeld hervorgehenden Quelle der Wissensproduktion scheint man es zunächst mit einer Umkehrung des Theorie-Praxis-Verhältnisses zu tun zu bekommen, aber damit würde man die entscheidende Pointe übersehen. Zwar verlagert sich die Produktion von Wissen gerade in den innovativen Gesellschaftsbereichen der späten Moderne, wie z. B. der IT-Technologie oder in systemischen Verfahren der Organisationsberatung und der Personalentwicklung sowie in der Urbanistik (Dell 2016), aus den tradierten Institutionen heraus in lebensweltnahe Formationen, was gegenwärtig an Phänomenen der „Entgrenzung“ von Institutionalformen in nahezu allen gesellschaftlichen Funktionssystemen beobachtet werden kann. So handelt es sich - um dies an einem Beispiel zu verdeutlichen - bei vielen theoretischen Ansätzen der Organisationstheorie um Reimporte aus Praxisfeldern der Entwicklungsberatung zurück in disziplinär eingehegte und universitär verwaltete Wissensbestände, die zuvor aus einer Theoriegenerierung in Praxiskontexten wissenschaftlicher Beratung (Weber 2005; Dell 2016) hervorgegangen waren, ohne allerdings dem Denkstil oder der Tradition einer einzelnen Disziplin verpflichtet zu sein. Exemplarisch hierfür können die Forschungsarbeiten von Karl Weick gelten, deren Theoreme transdisziplinäre Generalisierungen von Einsichten darstellen, die in kontextgebundener Problemanalyse in feldspezifischer Beratungspraxis gewonnen wurden (Weick 1985, 1995, 2001). Interessant ist hierbei, dass eine solche Rückführung in disziplinäres Wissen immer dort misslingen muss, wo sich aufgrund des grenzübergreifenden Charakters der in praktischen Verwendungszusammenhängen generierten Theorien keine dafür geeignete Disziplin für zuständig erklärt bzw. wo beim Reimport das neue Wissen disziplinär verengt und aufgrund epistemologisch differenter Hintergrundannahmen unzutreffend rezipiert und tradiert wird. Dies könnte auch für eine Bildungswissenschaft lebensbegleitenden Lernens zutreffen und führte zu der Konsequenz, dass ihre Engführung auf Erziehungswissenschaft überwunden und sie vielmehr auf einer transdisziplinären Ebene als Meta-Wissenschaft verstanden und weiterzuentwickeln wäre (vgl. Lenzen 1997). Im Verständnis einer „aufgabenorientierten Wissenschaft“ (Ostermeyer/Krüger 2015) im „Mode 2“ könnte auch das disziplinär chronifizierte Theorie-Praxis-Schisma auf einer epistemologischen Ebene einer relationstheoretischen Lösung zugeführt werden.

\section{4 „Aktionsforschung“, ein deutsches Erkenntnishindernis}

Die hier angeregte bildungswissenschaftliche Rezeption des internationalen Diskurses zum „mode 2 “ könnte auf erhebliche Abwehr stoßen, wenn das neue Verhältnis zwischen Wissenschaft und Gesellschaft kurzschlüssig mit dem in Deutschland in Verruf geratenen Konzept der Aktionsforschung gleichgesetzt würde. Während man im deutschsprachigen sozialwissenschaftlichen Diskurs zur Aktionsforschung unter diesem Begriff überwiegend ein inzwischen historisch „abgeschlossenes Kapitel“ (Altrichter/Gstettner 1993) versteht, das sich aufgrund methodologischer Inkonsistenz und geringer Fruchtbarkeit als obsolet erwiesen hat, wird der Begriff „Action Research“ in den angelsächsischen und skandinavischen Forschungsdiskursen auch über die traditionellen Konzepte hinausgehend weitergeführt und breit ausdifferen- 
ziert (vgl. Nielsen/Svensson 2006; Eikeland 2006, 2007, 2017). „Action Research“ bezieht sich unter der erweiterten Bedeutung von "Interactive Research" in einem umfassenderen Verständnis als in der deutschsprachigen Aktionsforschung der 7oer Jahre nun auch auf koproduktive Formen reziproker Zusammenarbeit zwischen dem Wissenschaftssystem und seiner gesellschaftlich-politischen Umwelt. Allerdings enthält sie (noch) nicht den von uns bildungstheoretisch gestalteten Zusammenhang einer methodologischen Verschränkung von Forschung und Lernen. Aufgrund dieser Differenz verzichten wir hier darauf, das von uns andernorts präsentierte und praktizierte Konzept einer „lernförderlichen Entwicklungsbegleitung“ begrifflich der Aktionsforschung zuzuordnen, selbst wenn diese neuerdings, ähnlich wie dies in den Schriften von Olav Eikeland erfolgt, nicht mehr als externe Interventionsstrategie, sondern gleichermaßen koproduktiv angelegt ist. Mögliche Gemeinsamkeiten und signifikante Unterschiede müssen daher einer späteren Diskussion überlassen bleiben (zur deutschsprachigen Kontroverse um Aktionsforschung vgl. Haag/Krüger/Schwärzel 1972; Altrichter/Gstettner 1993; Kordes 1995; Wolff 2008; Schäffter 2017).

\section{Forschung im Modus einer lernförmig angelegten Wissensproduktion}

Wenn wissenschaftliche Weiterbildung zur Schnittstelle zwischen disziplinären Wissensbeständen und tätigkeitsfeldgebundener Expertise werden soll, so hat sie sich einer intermediären Funktion zwischen zwei autonomen Wissensdomänen zu vergewissern. Die Deutung von Wissensdifferenz als Koproduktivität (Ibert/Müller/ Stein 2014) führte in Kontexten von „Bildung als Medium von Praxisforschung“ zur Entwicklung von Designs einer wissenschaftlichen Begleitung von Entwicklungsund Gestaltungsprojekten und stellte hierbei eine zentrale Fragestellung im Beraterdiskurs bei der Herausbildung praxisorientierter „Begleitforschung“ dar (vgl. Brödel et al. 2003; Weber 2005; Schäffter 2017). Als ein entscheidender Ertrag kann hierbei angesehen werden, dass die bisherige Dichotomie im Theorie-Praxis-Verhältnis überwunden werden und einem komplementären Verständnis Platz machen konnte. Dennoch stellten die Beratungskonzepte im Rahmen des Projekts „Lernkultur: Kompetenzentwicklung“ zunächst nur eine tentative Suchbewegung dar. Auch wenn die unterschiedlichen Prozesse wissenschaftlich angeleiteter Projektbegleitung eine derartige intermediäre Rolle einzunehmen pflegten, war diese „schwebend“ angelegte Positionierung von zielgenerierenden Planungskonzeptionen noch nicht hinreichend grundlagentheoretisch gesichert, um sie konzeptionell nach innen und außen artikulieren und somit auch bildungstheoretisch nachdrücklich vertreten $\mathrm{zu}$ können (vgl. ausführlich Schäffter 2017). 


\subsection{Verfahren und methodische Schritte einer transdisziplinär angelegten Praxisforschung}

Im Gegensatz zu disziplinärer Forschung, die ihren Ausgang an einer anschlussfähigen Problematik nimmt, die Bestandteil eines kontinuierlichen Entwicklungsverlaufs ist und damit als weitere Stufe eines fortschreitenden Erkenntniszusammenhangs verstanden werden kann, unterliegt eine transdisziplinär angelegte Praxisforschung einer grundsätzlich anderen Entwicklungslogik. Um sie zu verstehen, reicht es allerdings nicht aus, sie allein als Umkehrung zu betrachten, sondern ihr Verhältnis zu den für sie als relevant erscheinenden Wissenschaftsdisziplinen besteht in einer wechselseitig ausregelnden Abstimmung zwischen einer lebensweltlich bedeutsamen Verwendungssituation und einer auf diese bezogenen Theoriebildung. Ein derartiger Verlauf des Forschungsprozesses lässt sich in einer idealtypischen Schrittfolge beschreiben:

- Ausgang des Forschungsprozesses bildet eine sich manifestierende Problematik oder eine Entwicklungsoption in der Alltagspraxis, die einerseits theoretisch noch nicht hinreichend verstanden und zudem professionell noch unzureichend bearbeitet bzw. vorangetrieben werden kann. Hierbei geht es nicht zwingend um zu behebende Defizite, sondern vielmehr um sich neu herausbildende Möglichkeiten zur Welterschließung, also letztlich um Innovation.

- Die so erkannte Optionalität wird als Unterbrechung von Routineverläufen und damit als potenzieller Lernanlass genutzt, sofern überhaupt ein Entwicklungsspielraum („slack“) gegeben ist. In Anlehnung an John Dewey lässt sich formulieren, dass in diesem Fall auf eine Irritationserfahrung mit einer „Inquiry“ geantwortet wird, die in eine selbstgesteuerte Suchbewegung übergeht.

- Im Gegensatz zum traditionellen Konzept der „Professionswissenschaft“, bei dem sich eine spezielle Disziplin herausbildet, die sich für die Klärung und fachliche Bewältigung zukunftsrelevanter Entwicklungsprobleme als zuständig erklärt und somit eine Reflexionsfunktion übernimmt, braucht Praxisforschung in dem von Gibbons u. a. beschriebenen „mode 2 “ nicht mehr auf nur eine Referenzdisziplin zurückzugreifen, sondern bewegt sich bereits bei der „Situationsdefinition“ in einem transdisziplinären Rahmen. Im Prozess der „Inquiry“ stellt sich bereits die Frage, auf welchen disziplinären Deutungshorizont zur Problembeschreibung sinnvollerweise zurückgegriffen werden sollte. Eine solch transdisziplinäre Entscheidungsebene entspricht einer postmodernen Wissenschaftsauffassung (Moebius/Reckwitz 2008).

- Die im Verlauf der Inquiry vollzogene pragmatische Suchbewegung in Richtung auf eine adäquate „Situationsdefinition“ stellt sich nicht unter dem Wissenschaftscode von „Wahrheit“, sondern folgt den lebensweltlichen Relevanzen des jeweiligen Praxisfeldes und bleibt damit kontextgebunden. Aus dieser Perspektive werden nun wissenschaftliche Deutungsangebote aus einer oder mehreren Disziplinen herangezogen und in Hinblick auf ihre jeweilige Eignung durchmustert. In diesem Prozess erfährt die bisherige praxisinterne Situations- 
definition eine Horizonterweiterung und Ausdifferenzierung durch eine Außenperspektive.

- Im Aneignungsprozess zwischen einer zunächst konventionellen Situationsdefinition des Praxisfeldes und ihrer Horizonterweiterung durch externe Theoreme, die aus dem Relevanzsystem des Praxisfeldes als problemadäquat angesehen werden, bildet sich synergetisch ein neues Wissen heraus, das weder allein dem ursprünglich üblichen Praxiswissen noch den rezipierten disziplinären Wissensbeständen entspricht. Dies gilt insbesondere dann, wenn zur Problemklärung und seiner Lösung Theoreme und Wissensbestände aus unterschiedlichen Disziplinen herangezogen wurden, die zuvor unverbunden waren und nur unter der praxisbezogenen Problemstellung aufeinander bezogen wurden.

- Der transdisziplinär angelegte Aneignungsprozess aus der kritischen Interessenlage einer verwendungsbasierten Praxisforschung (vgl. Ostermeyer/Krüger 2015) stellt für alle daran beteiligten Akteursgruppen im Praxisfeld ein komplexes Projekt wissenschaftlicher Weiterbildung dar, gleichgültig ob sie personell den Relevanzen des Wissenschaftssystems oder denen des Praxisfeldes verpflichtet sind. Grundsätzlich handelt es sich um eine produktive Schnittstelle zwischen zwei oder mehreren Wissensdomänen, die in ihrer wechselseitigen Bezugnahme in einen transdisziplinären Bildungsprozess verwickelt werden, der nur gelingt, wenn sich beide Seiten in ihrer jeweiligen Position wertschätzen.

- Im Ergebnis führt ein derartig dialogischer Kontakt zur Generierung einer reflektierten Binnensicht und im Falle ihres Gelingens zu Lösungen, die nur eine der beiden Seiten aus ihren Bordmitteln heraus allein nicht erzielen könnte. Es handelt sich somit um innovatives Praxiswissen von wissenschaftlicher Qualität, auf das aufgrund ihrer „interaktiven Wertschöpfung“ von keiner der beteiligten Seiten alleiniger Anspruch erhoben werden kann. Ein solch dialogisch generiertes Praxiswissen hat den Charakter von Gemeingut (Helfrich/HeinrichBöll-Stiftung 2009).

\section{Hochschulpolitische Entwicklungsperspektiven}

Sobald Einrichtungen der wissenschaftlichen Weiterbildung ihre intermediäre Position zwischen Wissenschaft und Gesellschaft in der Weise erweitern, dass sie neben dem bisher praktizierten Wissenstransfer auch am institutionellen Zentralwert des Wissenschaftssystems, nämlich an der Forschung durch Theoriebildung und Wissensproduktion mitwirken, wird sich ihr Stellenwert besonders in den Hochschulen, aber auch in den Universitäten fundamental verändern. Die Mitwirkung an einer, alte Grenzen überschreitenden, Forschungspraxis, die gegenwärtig dabei ist, aus den herkömmlichen Institutionalformen des Wissenschaftssystems „auszuwandern“, führt sie aus einer peripheren Lage geradewegs ins Zentrum einer Entwicklung, von der Universitäten und Hochschulen aufgrund ihrer segmentierten Fächergliederung oder disziplinären Grenzregime bisher kaum ernsthaft Kenntnis genommen haben. 
Dem Selbstverständnis wissenschaftlicher Weiterbildung als Ausdruck einer sich transdisziplinär verstehenden Bildungswissenschaft (Lenzen 1997) käme hierbei auch zugute, dass sie sich allein durch ihre Mitwirkung an der Produktion von praxisfeldbasiertem Wissen von den anderen Institutionalformen des Bildungssystems dezidiert unterscheiden würde, die ja ebenfalls wissenschaftliche Weiterbildung betreiben, hierbei aber auf externe Wissensquellen zurückgreifen. Schließlich kommt ein gesellschaftspolitischer Aspekt hinzu: Die gesellschaftliche Inklusion in das Wissenschaftssystem (Stichweh 1988), die aufgrund eines rekursiven Leistungsprofils disziplinär segmentierter Theoriebildung letztlich nur für den Erkenntniszuwachs in der je eigenen Disziplin forscht und daher definitionsgemäß nur sekundäre, d.h. wissenschaftsfremde Formen eines gesellschaftlichen „Publikums“ aufbauen konnte, gewinnt nun über Forschungsmethoden „interaktiver Wertschöpfung“ in einer „Forschungswende“ (oekom 2015) die demokratische Bedeutung von Wissenschaft als ein „Common Good“ (Helfrich/Heinrich-Böll-Stiftung 2009) zurück. Damit eröffnen sich Entwicklungsperspektiven in Richtung auf ein Verständnis von „Citizen Science“ (Finke 2014; 2015), das die an der Forschung aktiv mitwirkenden PraktikerInnen und sich professionalisierenden Akteure in der Ökologiebewegung (oekom 2014) nicht nur als unbezahlte „Laien“ partizipieren, sondern sie in der Position von professionell arbeitenden Akteuren eines gesellschaftlichen Entwicklungsbereichs zu Initiatoren von zivilgesellschaftlich verankerter Praxisforschung werden lässt, zu der sie als AuftraggeberInnen und Akteure von Praxisforschung ihnen geeignet erscheinende WissenschaftlerInnen hinzuziehen. Wissenschaftliche Weiterbildung erhielte in einem partizipatorisch angelegten Wissenschaftsverständnis eine intermediäre Position, deren Wirkungen weit in den traditionellen Wissenschaftsbetrieb hineinreichen und dort auch intern eine Neuorientierung in Richtung auf eine „transformative Wissenschaft“ ermutigen und fachlich befördern könnten.

Für den zukünftigen wissenschaftlichen Status der Hochschulen und Fachhochschulen eröffnen die hier vorgetragenen Überlegungen ein weitgehend neues Kapitel. Zwar besteht zunächst Anlass zur Sorge, dass der sich gegenwärtig abzeichnende Strukturwandel von dieser dezidiert intermediären Institutionalform des Wissenschaftssystems ungenutzt bleibt, wenn man sich weiterhin unreflektiert an den Traditionen und Vorrechten der Universitäten orientiert. Andererseits zeigt sich am Material einer institutionstheoretisch angelegten empirischen Analyse (vgl. Schulze 2016), dass die Hochschulen im Vergleich zu den Universitäten bereits wichtige Voraussetzungen zur Transdisziplinarität mitbringen und - wenn auch weitgehend implizit - ihre Forschungsansätze nach dem oben skizzierten Verlauf eines „mode 2“ strukturieren. Insofern hat an den Hochschulen dieses „Neudenken“ in Richtung auf eine „aufgabenorientierte Wissenschaft“ schon lange eingesetzt. Es fragt sich nur, ob es auch explizit seine politische und institutionelle Anerkennung und methodologisch konsistente Berücksichtigung erfährt. Zudem wird eine strukturell entscheidende Differenz zwischen dem wissenschaftspolitischen Stellenwert von Einrichtungen wissenschaftlicher Weiterbildung im Kontext einer Universität und dem einer Hochschule erkennbar: Während wissenschaftliche Weiterbildung an den Hochschulen 
zu einem konstitutiven Bestandteil lernhaltiger Praxisforschung ausgebaut und institutionalisiert werden könnte und dies in ersten Ansätzen möglicherweise bereits der Fall ist, so bildet sie für universitäre Kontexte eine nur zusätzliche, aber begründungsbedürftige Option für transdisziplinäre Praxisforschung und damit gewissermaßen ein strukturelles Gegengewicht gegen die weiterhin fortschreitende Parzellierung in sich segmentär abgrenzende Teildisziplinen. Diese Strukturdifferenz genauer zu begründen und in ihren hochschulpolitischen Entwicklungsperspektiven zu entfalten, fehlt hier der Raum. Ihre weitere Diskussion in den Einrichtungen der wissenschaftlichen Weiterbildung anzustoßen, war letztlich Anlass und Herausforderung zu diesen Überlegungen.

\section{Literatur}

Altrichter, H./Gstettner, P. (1993): Aktionsforschung - ein abgeschlossenes Kapitel in der Geschichte der deutschen Sozialwissenschaft? In: Sozialwissenschaftliche LiteraturRundschau.

Bachmann-Medick, D. (2014): Cultural Turns: Neuorientierungen in den Kulturwissenschaften. Reinbek.

Bachmann-Medick, D. (Hrsg.) (1997): Übersetzung als Repräsentation fremder Kulturen. Berlin.

Beck, U./Bonß, W. (1989): Weder Sozialtechnologie noch Aufklärung? Analysen zur Verwendung sozialwissenschaftlichen Wissens. Frankfurt am Main.

Bender, G. (Hrsg.) (2001): Neue Formen der Wissenserzeugung. Frankfurt am Main/New York.

Böhmer, A. (2014): Diskrete Differenzen. Experimente zur asubjektiven Bildungstheorie in einer selbstkritischen Moderne. Bielefeld.

Bonnemeier, S./Reichwald, R. (2012): „Hybride Wertschöpfung“ - vom Industrieproduzenten zum Lösungsanbieter - ein State-of-the-Art Bericht. In: Zeitschrift für betriebswirtschaftliche Forschung (zfbf), Sonderheft 65, S. 45-72.

Brödel, R./ Bremer, H./Chollet, A./Hagemann, I.-M. (Hrsg.) (2003): Begleitforschung in Lernkulturen. Münster.

Burzan, N./Lökenhoff, B./Schimank, U./Schöneck, N. M. (2008): Das Publikum der Gesellschaft. Inklusionsverhältnisse und Inklusionsprofile in Deutschland. Wiesbaden: VS Verlag.

Deutscher Gewerkschaftsbund (DGB): Fuer-eine-demokratische-und-soziale-Hochschulehochschulpolitisches-Programm-des-DGB.pdf http://www.dgb.de/themen/++co++619 26a20-4053-11e2-a408-00188b4dc422

Dell, C. (2016): Epistemologie der Stadt. Improvisatorische Praxis und gestalterische Diagrammatik. Bielefeld. 
Dewe, B. (1988): Wissensverwendung in der Fort- und Weiterbildung. Zur Transformation wissenschaftlicher Informationen in Praxisdeutungen. Baden-Baden.

Eikeland, O. (2017): Die Epistemologie oder Gnoseologie der Aktionsforschung. Erscheint in: Schemme, D./Novak, H. (Hrsg.): Gestaltungsorientierte Forschung in Innovationsund Entwicklungsprogrammen - Potenzial für Praxisgestaltung und Wissenschaft. Bonn: BIBB.

Eikeland, O. (2007): From epistemology to gnoseology - understanding the knowledge claims of action research. In: Management Research News, 5, S. 344-358.

Eikeland, O. (2006): Validity of Action Research and Validity in Action Research. In: Nielsen, K. A./Svensson, L. (Hrsg.): Action and Interactive Research. Beyond practice and theory. Maastricht, S. 193-240.

Finke, P. (Hrsg.) (2015): Freie Bürger - Freie Forschung. Die Wissenschaft verlässt den Elfenbeinturm. München: oekom.

Finke, P. (Hrsg.) (2014): Citizen Science. Das unterschätzte Wissen der Laien. München: oekom.

Funtowicz, S./Ravetz, J. (2010): Post-Normal Science and Governance und Conditions of Complexity. In: Decker, M. (Hrsg.): Interdisciplinarity in Technology Assessment. Implementation and its Chances and Limits. Berlin/Heidelberg et al., S.15-24.

Gibbons, M. (2000): Context-Sensitive Science. In: Science and Public Policy, 3, S. 159-163. http://supportservices.ufs.ac.za/dl/userfiles/Documents/o0ooo/546_eng.pdf

Gibbons, M./Limoges, C./Nowotny, H./Schwartzmann, S./Scott, P./Trow, M. (1994): The New Production of Knowledge. The Dynamics of Science und Research in Contemporary Societies. London.

Haag, F./Krüger, H./Schwärzel, W./Wildt, J. (Hrsg.) (1972): Aktionsforschung. Forschungsstrategien, Forschungsfelder und Forschungspläne. München.

Helfrich, S. /Heinrich-Böll-Stiftung (Hrsg.) (2009): Wem gehört die Welt? Zur Wiederentdeckung der Gemeingüter. München/Berlin.

Hellmann, K.-U. (2010): Prosumer Revisited: Zur Aktualität einer Debatte. Eine Einführung. In: Blättel-Mink, B./Hellmann, K.-U. (Hrsg.): Prosumer Revisited. Zur Aktualität einer Debatte. Wiesbaden: VS Verlag.

Höhne, S. (2009): Kunst- und Kulturmanagement. Eine Einführung. München: Fink.

Ibert, O./Müller, F. C./Stein, A. (2014): Produktive Differenzen. Eine dynamische Netzwerkanalyse von Innovationsprozessen. Bielefeld: transcript.

Kordes, H. (1995): Pädagogische Aktionsforschung. In: Enzyklopädie Erziehungswissenschaft. Band 2, Stuttgart, S. 185-219.

Lenzen, D. (1997): Lebenslauf oder Humanontogenese? Vom Erziehungssystem zum kurativen System - von der Erziehungswissenschaft zur Humanvitiologie. In: Lenzen, D. Luhmann, N. (Hrsg.): Bildung und Weiterbildung im Erziehungssystem. Lebenslauf und Humanontogenese als Medium und Form. Frankfurt am Main: Suhrkamp, S. 228-247.

Meier-Rabler, U. (1990): Strukturwandel der Wissensproduktion. Das Ende der Wissensmonopole? Salzburg. http://www.inst.at/trans/6Nr/maier.htm 
Moebius, S./Reckwitz, A. (Hrsg.) (2008): Poststrukturalistische Sozialwissenschaften. Frankfurt am Main: Suhrkamp.

Nielsen, K. A./Svensson, L. (Hrsg.) (2006): Action and Interactive Research. Beyond practice and theory. Maastricht.

Nowotny, H. (1999): Es ist so. Es könnte auch anders sein. Über das veränderte Verhältnis von Wissenschaft und Gesellschaft. Frankfurt am Main.

Nowotny, H./Scott, P./Gibbons, M. (2014): Wissenschaft neu denken. Wissen und Öffentlichkeit in einem Zeitalter der Ungewissheit. Weilerswist: Velbrück.

Nowotny, H./Scott, P./Gibbons, M. (2001): Re-Thinking Science. Knowledge and the Public in the Age of Uncertainty. Cambridge.

Oekom e. V. (2015): Forschungswende. Wissen schaffen für die Große Transformation. München: oekom.

Ostermeyer, S. P./Krüger, S.-K. (Hrsg.): Aufgabenorientierte Wissenschaft. Formen transdisziplinärer Versammlung. Münster/New York.

Reckwitz, A. (2004): Die Kontingenzperspektive der Kultur. Kulturbegriffe, Kulturtheorien und das kulturwissenschaftliche Forschungsprogramm. In: Jaeger, F./Rüsen, J. (Hrsg.): Handbuch der Kulturwissenschaften. Band 3. Stuttgart/ Weimar, S. 1-20.

Reichwald, R./Piller, F. (2006): Interaktive Wertschöpfung. Open Innovation, Individualisierung und neue Formen der Arbeitsteilung. Wiesbaden: Gabler.

Reichwald, R./Krcmar, H./Nippa, M. (Hrsg.): Hybride Wertschöpfung: Konzepte, Methoden und Kompetenzen für die Preis- und Vertragsgestaltung. Lohmar.

Renn, J. (2002): Einleitung: Übersetzen, Verstehen, Erklären. Soziales und sozialwissenschaftliches Übersetzen zwischen Erkennen und Anerkennen. In: Renn, J./Shimada, S./Straub, J. (Hrsg.): Übersetzung als Medium des Kulturverstehens und sozialer Integration. Frankfurt am Main/New York, S. 13-35.

Rheinberger, H.-J. (2007): Historische Epistemologie zur Einführung. Hamburg: Junius.

Rosa, H. (2016): Resonanz. Eine Soziologie der Weltbeziehung. Berlin: Suhrkamp.

Schaaf, J. (o. J.): Heidegger und die Ökologie. http://haus-des-verstehens.ch/metaphysik frei-reloaded/219.html

Schaaf, J. (1966): Beziehung und Beziehungsloses (Absolutes). In: Henrich, D./Wagner, H. (Hrsg.): Subjektivität und Metaphysik. Festschrift für Wolfgang Cramer. Frankfurt am Main: Klostermann, S. 277-289.

Schäffter, O. (2017): Bildung im Medium von Forschung - Forschung im Medium von Bildung. Strategien einer lernförderlich gestaltenden Entwicklungsbegleitung. Erscheint in: Schemme, D./Novak, H. (Hrsg.): Gestaltungsorientierte Forschung in Innovations- und Entwicklungsprogrammen - Potenzial für Praxisgestaltung und Wissenschaft. Bonn: BIBB.

Schäffter, O. (2014): Die Kategorie der Relationalität - der paradigmatische Kern und einzelwissenschaftliche Anwendungsfelder. Version 18 vom o8.01.2014. https://ebwb.huberlin.de/team/schaeffter/katrel_16_1 
Schäffter, O. (2012): Relationale Weiterbildungsforschung. In: Baldauf-Bergmann, K. (Hrsg.): Veränderungen von Lernen und Weiterbildung in der Transformationsgesellschaft. Tätigkeitstheorie. Journal für tätigkeitstheoretische Forschung in Deutschland (Online-Journal) 2012(7). http://www.ich-sciences.de/index.php?id=87\&L=0

Schäffter, O. (2011): Die Kontingenzperspektive auf den Forschungsgegenstand. In: Hof, C./Ludwig, J./Schäffer, B. (Hrsg.): Steuerung - Regulation - Gestaltung. GovernanceProzesse in der Erwachsenenbildung zwischen Struktur und Handlung. Baltmannsweiler: Schneider, S. 232-239. https://ebwb.hu-berlin.de/team/schaeffter/downloads/iii88

Schäffter, O. (2001): Weiterbildung in der Transformationsgesellschaft. Zur Grundlegung einer Theorie der Institutionalisierung. Baltmannsweiler: Schneider.

Schneidewind, U./Singer-Brodowski, M. (2014): Transformative Wissenschaft. Klimawandel im deutschen Wissenschafts- und Hochschulsystem. 2. Aufl. Marburg: Metropolis.

Schulze, M. (2016): Erfolgsfaktoren weiterbildender Masterstudiengänge an Fachhochschulen. Der berufsbegleitende weiterbildende Masterstudiengang Sozialmanagement. Unveröff. Manuskript einer sich in Arbeit befindlichen Dissertation an der HU Berlin.

Stichweh, R. (1988): Inklusion in Funktionssysteme der modernen Gesellschaft. In: Mayntz, R./Rosewitz, B./Schimank, U./Stichweh, R. (Hrsg.): Differenzierung und Verselbständigung. Zur Entwicklung gesellschaftlicher Teilsysteme. Frankfurt/New York: Campus, S. 293.

Strathern, M. (2004): Commons and Borderlands: Working Papers on Interdisciplinarity, Accountability and the Flow of Knowledge.

Weber, C. (2005): Koproduktive Prozesse: Wissensnetzwerke zwischen kontextualisiertem und dekontextualisiertem Wissen. In: Baldauf-Bergmann, K./Küchler, F. von/ Weber, C. (Hrsg.): Erwachsenenbildung im Wandel - Ansätze einer reflexiven Weiterbildung. Baltmannsweiler: Schneider, S. 125-135.

Weick, K. E. (2001): Drop your Tools. In: Bardmann, T. M./Groth, T. (Hrsg.): Zirkuläre Positionen 3. Organisation, Management und Beratung. Opladen, S. 123-138.

Weick, K. E. (1995): Sensemaking in Organizations. Thousand Oaks.

Weick, K. E. (1985): Der Prozess des Organisierens. Frankfurt am Main.

Weingart, P. (2015): Die Wissenschaft der Öffentlichkeit. Essays zum Verhältnis von Wissenschaft, Medien und Öffentlichkeit. Weilerswist: Velbrück.

Weingart, P. (2005): Die Stunde der Wahrheit? Zum Verhältnis der Wissenschaft zu Politik, Wirtschaft und Medien in der Wissensgesellschaft. Weilerwist: Velbrück.

Wingens, M./Weymann, A. (1991): Die Verwendung soziologischen Wissens in der bildungspolitischen Diskussion. Pfaffenweiler.

Wolff, S. (2008): Wie kommt die Praxis zu ihrer Theorie? Über einige Merkmale praxissensibler Sozialforschung. In: Kalthoff, H./Hirschauer, S./Lindemann, G. (Hrsg.): Theoretische Empirie. Zur Relevanz qualitativer Forschung. Frankfurt am Main: Suhrkamp, S. 234-259.

Wolzogen, C. von (1992): Eintrag Relation im 20.Jahrhundert. In: Ritter, J./ Gründer, K. (Hrsg.): Historisches Wörterbuch der Philosophie. Bd. 8, Darmstadt: WBG, S. 602-6o6.

Ziman, J. M. (1996): „Postacademic Science“: Constructing Knowledge with Networks and Norms. In: Science Studies, 1, S. 67-80. 
Empfehlungen der DGWF 



\title{
Organisation der wissenschaftlichen Weiterbildung an Hochschulen ${ }^{1}$
}

\author{
DGWF-Empfehlung, beschlossen am 16./17.07.2015 \\ in Oestrich-Winkel
}

\section{Ausgangslage}

Die Frage, wie die Weiterbildung an einer Hochschule idealerweise organisiert und vor allem institutionell verankert werden kann, hat nicht an Aktualität verloren, stellt sie sich doch insbesondere bei drei Anlässen, die auch in letzter Zeit häufig zu beobachten sind:

- Neugründung einer Weiterbildungseinrichtung, weil die Weiterbildung neu oder verstärkt als Aufgabe der Hochschule wahrgenommen wird oder aufgrund der Neugründung einer Hochschule.

- Reorganisation der Weiterbildung aufgrund eines veränderten Umfelds, eines veränderten Verständnisses von Weiterbildung oder einer Reorganisation der Hochschule insgesamt.

- Überführung von befristeten, über Programmmittel finanzierten Personalstellen in eine Daueraufgabe der Hochschule.

Im Rahmen eines DGWF-Projekts zur Organisation und Reorganisation wissenschaftlicher Weiterbildung haben Dollhausen, Ludwig und Wolter den politischen und institutionellen Hintergrund von Veränderungen in der wissenschaftlichen Weiterbildung an Hochschulen und damit die Ausgangslage für die vorliegenden Empfehlungen wie folgt beschrieben (2013, S.10-11):

„Seit einigen Jahren zeigen insbesondere zwei bildungspolitische Strategien in der deutschen Hochschullandschaft deutliche Effekte. $\mathrm{Zu}$ nennen ist zum einen der Ende der 1990er Jahre auf den Weg gebrachte Bologna-Prozess, der sowohl auf die ,Harmonisierung' der akademischen Erstausbildung durch die Einführung von Bachelor- und Masterstudiengängen sowie die Umsetzung von Punktesystemen zur Leistungsbewertung (ECTS) zielt, als auch auf die Erweiterung des Bildungs-

1 Ersetzt die Empfehlungen der DGWF von 2010: Status und Personal der Einrichtungen für Weiterbildung. Sie wurden redaktionell bearbeitet von Bernhard Christmann und Andreas Fischer. 
angebots der Hochschulen durch die Entwicklung und Einführung von berufsbegleitenden Bachelorstudiengängen, weiterbildenden Masterstudiengängen, Zertifikatsstudien und weiteren, kürzeren Angebotsformen für nicht regulär, d.h. in akademischer Erstausbildung und in Vollzeit Studierende. Zum anderen ist in den Hochschulen infolge der Verpflichtung der EU-Mitgliedstaaten auf die LissabonAgenda im Jahr 2000 und mithin der Förderung des lebenslangen Lernens als einem Schlüsselelement zur Erreichung des strategischen Ziels, Europa zur ,wettbewerbsfähigsten und dynamischsten Wissensgesellschaft der Welt‘ zu machen (EUKommission 2001, S. 3), ein Veränderungsbedarf entstanden. (...) Mindestens ebenso geraten die Hochschulen heute infolge des Greifens von neuen Steuerungsmodellen in der Hochschulverwaltung und in der Wissenschaft sowie eines Bedeutungszuwachses der Hochschulen im Rahmen von regionalen Entwicklungsstrategien unter einen Veränderungsdruck. (...) In den Hochschulen führt dies dazu, dass die eigene Entwicklung verstärkt unter Gesichtspunkten der Qualität und Effizienz, Attraktivität und Dienstleistungsorientierung, der wissenschaftlichen Exzellenz und der Vernetzung mit der Region reflektiert und forciert werden muss. Ein sich bereits abzeichnender organisatorischer Effekt dieser Entwicklung auf der Ebene der Hochschulen ist dabei eine (Re-)Zentralisierung von Aufgaben und Funktionen der strategischen Planung und Steuerung der Hochschulentwicklung, insbesondere eine deutliche Stärkung des Hochschulmanagements.

Die hier nur skizzierten Entwicklungen im Hochschulbereich sind für die Einrichtungen der wissenschaftlichen Weiterbildung an Hochschulen vor allem in zweierlei Hinsicht folgenreich: Einerseits erfährt die wissenschaftliche Weiterbildung, insbesondere im Zusammenhang mit den Öffnungserfordernissen des hochschulischen Lehr- bzw. Bildungsangebots im Kontext des Lebenslangen Lernens als Kernaufgabe der Hochschulen neben Forschung und Lehre, eine deutliche Aufwertung. Andererseits sind damit aber auch Bedeutungsverschiebungen im Verhältnis der wissenschaftlichen Weiterbildung und ihrer Einrichtungen zur Hochschulleitung sowie zu den Fakultäten, die die grundständige Lehre realisieren, angezeigt. Mit diesen strukturellen Veränderungen werden gegenwärtig viele Hochschulen in der Planung und Umsetzung von (Re-)Organisationsmaßnahmen der wissenschaftlichen Weiterbildung und ihrer Einrichtungen konfrontiert."

\section{Organisationsformen}

In der Länderstudie Deutschland der internationalen Vergleichsstudie zur Struktur und Organisation der Weiterbildung an Hochschulen (Hanft/Knust 2007) unterscheiden Faulstich et al. (a.a. O., S. 110) folgende Organisationsformen (in Reihenfolge der Häufigkeit):

- Wissenschaftliche Einrichtung/wissenschaftliches Zentrum,

- Zentrale Betriebseinheit,

- Abteilung/Arbeitsbereich in zentraler Verwaltung, 
- An-Institut,

- Außeruniversitäre Einrichtung,

- Stabsstelle/Stabsabteilung der Hochschulleitung,

- Teil einer Fakultät/eines Fachbereichs,

- (Sonstiges).

„Am häufigsten (jeweils mehr als $17 \%$ ) wird die Weiterbildung als wissenschaftliche Einrichtung/wissenschaftliches Zentrum, als zentrale Betriebseinheit oder als Abteilung/Arbeitsbereich in zentraler Verwaltung organisiert, gefolgt von der Organisation als An-Institut, als außeruniversitäre Einrichtung, als Stabsstelle oder als Teil einer Fakultät bzw. eines Fachbereichs (jeweils weniger als 10\%). Fast $15 \%$ agieren in anderen Organisationsformen (hier wurden hauptsächlich Vereine genannt)“ (ebd.).

Unabhängig von der Bezeichnung der Organisationsform, wie in den oben genannten Beispielen, können grundsätzlich und in systematischer Betrachtung die Verantwortlichkeiten für die Weiterbildung erstens innerhalb oder außerhalb der Hochschule angesiedelt werden. Bei einer Organisationsform innerhalb der Hochschule können sie zweitens in der Nähe der Wissenschaft oder der Verwaltung oder im dazwischenliegenden, intermediären „third space“ positioniert sowie eher zentral oder eher dezentral organisiert werden. Drittens ist bezüglich der Aufgabenbreite zwischen reinen Weiterbildungsstellen und solchen mit einem erweiterten Aufgabenspektrum zu unterscheiden.

Die Wahl der Organisationsform für die wissenschaftliche Weiterbildung ist prinzipiell abhängig von den inner- und außerhochschulischen Rahmenbedingungen, vom Aufgabenspektrum sowie von den zugeordneten Funktionen. Nicht zuletzt die Länderstudie Deutschland hat jedoch deutlich gemacht, dass die wissenschaftliche Weiterbildung institutionell in sehr unterschiedlicher Weise in die Hochschulen eingebunden ist. „Die strategischen Ansatzpunkte und Ausrichtungen der Re-Organisationsmaßnahmen hängen daher von der jeweiligen Einbettung bzw. Kopplung der wissenschaftlichen Weiterbildung in bzw. an den institutionellen Kontext der Hochschule sowie von bereits vorhandenen Entwicklungspfaden der Organisation wissenschaftlicher Weiterbildung ab“ (Dollhausen/Ludwig/Wolter 2013, S. 11).

Nachfolgend werden diese möglichen Organisationsformen sowie die Aufgabenbreite und Funktionen der wissenschaftlichen Weiterbildung näher betrachtet. Auf dieser Basis werden dann abschließend Empfehlungen für die Organisation wissenschaftlicher Weiterbildung formuliert.

\section{Strukturelle Verortung in der Hochschule}

Im Zuge der zunehmenden Ausdifferenzierung und Reorganisation der Hochschulen ist zwischen dem Wissenschaftsbereich und der Verwaltung ein intermediärer Bereich, der „third space“, entstanden. Diesem gehören Organisationseinheiten an, die weder rein wissenschaftlich noch administrativ ausgerichtet sind. „Die Organisa- 
tionsstrukturen der Wissenschaftsorganisationen, insbesondere der Universitäten, wurden sowohl nach innen als auch nach außen offener (Whitchurch 2004, S. 291). Dadurch ergaben sich für das Personal Möglichkeiten, Aufgabenbereiche neu und vor allem zunehmend eigenständig zu definieren. Es bildeten sich laut Whitchurch berufliche Rollen heraus, die weder eindeutig der Wissenschaft noch der Verwaltung zuzurechnen sind und deshalb einem third space angehören. Das dort tätige Personal übernimmt sowohl akademische wie auch administrative Funktionen und vermittelt zwischen den verschiedenen institutionellen Bereichen einer Wissenschaftsorganisation. Das Ausüben dieser Zwischenpositionen stellt z. T. hohe Anforderungen an Fähigkeiten und Qualifikationen dar. Da die Tätigkeiten die traditionellen Grenzen zwischen Wissenschaft und Administration überschreiten, kommt es zudem bei denjenigen, die traditionelle Berufsmodelle getrennt nach Wissenschaft und Verwaltung praktizieren, zu Irritationen und misstrauischem Verhalten. Die Entstehung des third space ist ein organisationskultureller Bruch, dessen Verarbeitung einige Zeit beanspruchen wird und eine hohe Ambiguitätstoleranz bei allen Beteiligten erfordert“ (Kottmann/Nickel 2010, S. 24).

Für das Profil und den Status der für die Weiterbildung einer Hochschule zuständigen Stelle ist es von großer Bedeutung, wo sie in diesem Feld verortet wird: akademisch/wissenschaftsnah, administrativ/verwaltungsorientiert oder intermediär?

\section{Primär wissenschaftlich ausgerichtete Stelle (in der Regel fakultätsnah situiert)}

Diese Option wird gewählt, wenn es sich im engeren disziplinären Sinne um Wissenschaft in Forschung und Lehre handelt; disziplin- und fakultätsübergreifende Leistungen sowie die Dienstleistungen werden angehängt.

Bezeichnungen: wissenschaftliche Einrichtung, wissenschaftliches Zentrum, Institut, Forschungsstelle.

- Vorteile: Forschung und Vermittlung liegen nah beieinander und profitieren voneinander. Wissenstransfer erfolgt ohne Umwege. Für Wissenschaftler/innen kann es attraktiv sein, sich an der Weiterbildung zu beteiligen. Die Weiterbildungsstelle profitiert vom Prestige einer wissenschaftlichen Einrichtung.

- Nachteile: Wissenschaftliche Einrichtungen werden in der Regel Fakultäten zugeordnet. Damit bedarf es eines besonderen kommunikativen Aufwandes, die disziplin- und fakultätsübergreifenden Leistungen zu platzieren und umzusetzen. Weiterbildung als Aufgabe besonderer Art, die sich von Forschung und Studium unterscheidet, wird oft schwer verstanden und dadurch behindert. Forschung hat Priorität gegenüber Weiterbildung.

Um die Sichtbarkeit der wissenschaftlichen Weiterbildung zu unterstützen, ist eine zentrale wissenschaftliche Einrichtung geeigneter, die dann keiner Fakultät mehr zugeordnet ist. Dies sind dann allerdings in der Regel eher intermediär ausgerichtete Stellen. 


\section{Primär administrativ ausgerichtete Stelle}

(in der Regel in die Verwaltung integriert)

Diese Option wird gewählt, wenn übergreifende Leistungen und Services im hochschuleigenen System mit einer engen Anbindung und Steuerung realisiert werden sollen bzw. im Vordergrund der zugedachten Aufgaben stehen. Dies kann in Form einer zentralen Betriebseinheit, aber auch innerhalb der Verwaltung oder als Stabsstelle erfolgen. Stabsstellen übernehmen eher Kommunikations- und Steuerungsfunktionen aus Sicht der Hochschulleitungen; Verwaltungseinheiten orientieren sich stark an der Organisation ordnungsgemäßer Abläufe - beide Formen bilden jeweils nur einen geringen Teil der Aufgabenvielfalt wissenschaftlicher Weiterbildung $\mathrm{ab}$.

Bezeichnungen: Fachstelle, Referat, Stabsstelle, Supportstelle, Abteilung, zentrale Betriebseinheit, Dienststelle.

- Vorteile: Die Verwaltungsnähe erleichtert die betrieblichen Abläufe der Finanzund Personaladministration und den Zugang zur Infrastruktur (Räume, EDV, Druck- und Kopierzentrale).

- Nachteile: Oft mangelhaftes Verständnis für die weiterbildungsspezifischen Anforderungen an die Administration, Abwehrreflexe der Wissenschaftler/innen, wenig Reputation, geringere Flexibilität.

\section{Intermediär ausgerichtete Stelle mit wissenschaftlichen und Dienstleistungs- Aufgaben}

(in der Regel in „neuen“ Organisationsformen zwischen den Fakultäten oder nahe der Hochschulleitung ohne Integration in Verwaltung)

Eine solche Stelle kann eher wissenschaftlich mit einer professoralen Leitung oder eher dienstleistend mit einem (wissenschaftlichen) Beirat ausgestaltet werden. Die professorale Leitung wird in der Regel über Deputatsfreistellungen ermöglicht.

Bezeichnungen: Zentrum, Kompetenzzentrum, Weiterbildungszentrum, Professional School.

Für intermediäre Einrichtungen ergibt sich der größte Handlungsspielraum insbesondere dann, wenn

- für das eigene Tätigkeitsfeld auch wissenschaftliche Schwerpunkte gesetzt werden,

- die wissenschaftliche Weiterbildung hochrangig in das Profil und das Leitbild der Hochschule eingebunden ist,

- fakultäts- und disziplinübergreifend vernetzt gearbeitet wird,

- eine strategische Unterstützung durch einen entsprechend besetzten Beirat erfolgt.

- Vorteile: Die Vorteile entsprechen denjenigen der primär wissenschaftlich ausgerichteten Stellen. Eine professorale Leitung erhöht das Renommee und die Reputation der Einrichtung - insbesondere dann, wenn die Professur entspre- 
chend besetzt ist. Die Stelle kann den ganzen Support aus einer Hand bieten. Sie ist legitimiert, in eigener Regie wissenschaftliche Weiterbildung anzubieten.

- Nachteile: Kritisch sind hier die Passung sowie das inhaltliche und personale Arrangement zwischen professoraler Leitung und operativer Geschäftsführung.

\section{Zentralität innerhalb der Hochschule}

Die Hochschulen müssen festlegen, wie sie die mit dem Angebot von Weiterbildung verbundenen Funktionen organisatorisch zuordnen. Soll alles an einem Ort konzentriert oder auf verschiedene Stellen aufgeteilt werden? Dabei ist auch über die Verteilung auf die verschiedenen Ebenen zu entscheiden (ganze Hochschule, Teilhochschule, Fakultät/Departement, Institut/Abteilung): Alles auf einer Ebene (und welcher?) oder auf welchen anderen Ebenen auch noch? Es ist davon auszugehen, dass es an vielen Hochschulen parallele Organisationformen gibt bzw. geben wird: eine zentrale Organisation und davon unabhängig dezentral organisierte Angebote einzelner Fakultäten/Fachbereiche.

- Vorteile zentraler Organisation: Weiterbildung kann gesteuert und strategisch ausgerichtet werden, einheitliche Bedingungen und einheitlicher Auftritt, Profilierung und Wiedererkennbarkeit, Möglichkeiten des finanziellen Ausgleichs (Querfinanzierung), Nähe zur Hochschulleitung/Macht, Effizienz/Skalenerträge, Professionalität, Managementstrukturen, leichte Integration in Qualitätssicherung.

- Vorteile dezentraler Organisation: Nähe zur programmspezifischen Wissenschaft und Forschung und der entsprechenden Wissenschaftskultur, Nähe zur Lehre und den Lehrenden und der entsprechenden didaktischen Kultur, Nähe und Zugang $\mathrm{zu}$ den Zielgruppen, kurze administrative und organisatorische Wege, Engagement und "Ownership“ der Programmverantwortlichen, flexible Lösungen.

\section{Beispiele}

- Zentrale Organisation auf der Ebene von Hochschule oder Fakultät: Das Weiterbildungszentrum ist für alle strategischen und operativen Funktionen verantwortlich, von der Entwicklung über Planung, Organisation und Bewerbung bis zur Durchführung und Evaluation.

- Dezentrale Organisation auf der Ebene der Institute mit zentraler Unterstützung der Ebene Hochschule: Die Programme werden durch die Institute entwickelt, geplant und angeboten, die Bewerbung und Teile der Organisation (Räume, Finanzen) werden zentral auf der Ebene Hochschule erledigt (oder andere Aufteilungen). 


\section{Auslagerung}

Die Aufgaben von Weiterbildungseinrichtungen werden in der Regel innerhalb der Hochschule erfüllt und im internen Organigramm der Hochschule verortet. Aus vor allem rechtlichen und finanziellen Gründen (insbesondere dienst-, haushalts- und besoldungsrechtliche Vorgaben) können diese Aufgaben aber auch ausgelagert werden, in erster Linie in sachlich und vertraglich eng mit der Hochschule verzahnte und von ihr kontrollierte privatrechtliche Organisationen wie Vereine, GmbH, AnInstitute, Akademien und Aktiengesellschaften (vgl. Faulstich et al. in Hanft/Knust 2007, S.109).

- Vorteile der Auslagerung: größerer Handlungsspielraum auf dem Weiterbildungsmarkt (Profil, Label, Werbung, Preis- und Produktgestaltung, Verpflichtung externer Lehrpersonen aus der Praxis, Kooperationsmöglichkeiten mit Organisationen der Nachfrage), Freiheit in den Anstellungs- und Entlohnungsbedingungen des Lehrpersonals, geeignete Infrastruktur und ggf. günstigerer Standort.

- Nachteile der Auslagerung: Gefahr der zunehmenden Abkoppelung (Entfernung von der Mutterhochschule), schwierigere Durchsetzung der Standards der Hochschule (Qualitätssicherung), Legitimation zum Angebot wissenschaftlicher Weiterbildung muss immer wieder bekräftigt werden, geringe Identifikation der Lehrenden und Forschenden der Hochschule mit ihrer Weiterbildung.

- Anbindungsformen:

- Eigenständige Einrichtung unter Aufsicht der Hochschule,

- Eigenständige Einrichtung mit Beteiligung und Mitsprache der Hochschule,

- Eigenständige Einrichtung ohne Mitsprache der Hochschule,

- An-Institut mit enger organisatorischer und personeller Koppelung.

- Rechtsformen der Auslagerung:

- Verein,

- $\mathrm{GmbH}$ (eventuell gemeinnützig),

- Stiftung,

- AG,

- An-Institut (i.d. R. als GmbH).

\section{Aufgabenbreite und Funktionen der Einrichtung}

Die Wahl der Organisationsform ist außer von externen und internen rechtlichen Rahmenbedingungen vorrangig abhängig von dem der Einrichtung zugedachten Aufgabenspektrum und den damit verbundenen Funktionen im Gesamtgefüge der Organisation und Leistungen der Hochschule. Je ausgeprägter und eigenständiger diese Funktionen und Aufgabenbereiche formuliert werden, umso eher lässt sich eine geeignete Organisationsform begründen. 
Durch eine interne Reorganisation des Wissenschaftsbereichs wird oft das Verhältnis der Fachbereiche bzw. Fakultäten zur Hochschulleitung und Verwaltung verändert. Ebenso können durch eigenständigere Aktivitäten der Fakultäten einige zentrale Funktionen umgelagert werden. Daher muss dann die Institutionalisierung bzw. Reorganisation der Einrichtungen für Weiterbildung zwischen dezentralen Wissenschaftsaktivitäten und zentralen Leitungs- und Steuerungsfunktionen neu bestimmt werden. Zunächst geht es darum, ihre Zuständigkeiten zu redefinieren. Dabei muss unterschieden werden zwischen den Funktionen wissenschaftlicher Weiterbildung einerseits und den Aufgaben und der Organisation der einschlägigen Einrichtung für Weiterbildung an Hochschulen andererseits.

Zentrale Aufgaben der Einrichtungen für wissenschaftliche Weiterbildung, welche von anderen Institutionalisierungsformen im Wissenschaftsbereich nicht erfüllt werden, beziehen sich hauptsächlich auf das „Bildungsmanagement“ wissenschaftlicher Weiterbildung. Dies ist nicht nur eine betriebswirtschaftliche, sondern vor allem eine bildungswissenschaftliche Aufgabe, die gefasst werden kann unter dem Begriff der Makrodidaktik, wenn es darum geht, die Rahmenbedingungen für das Lernen wissenschaftlichen Wissens zu organisieren. Neben den organisatorischen oder administrativen Aufgaben ergeben sich aus diesem Profil auch eigenständige wissenschaftliche Aufgaben, welche auch Forschungsaktivitäten implizieren können.

Demgemäß erfüllen Einrichtungen für Weiterbildung für die Hochschulen idealerweise eine Reihe von Funktionen:

- die disziplin- und/oder fakultätsübergreifende Bearbeitung von Problemstellungen und Beratung der Hochschule,

- die Entwicklung und Erschließung von Themen,

- die Erschließung von Zielgruppen,

- die Umsetzung von Bildungsveranstaltungen,

- Innovationen bei der Organisation, bei Methoden und Problemperspektiven,

- den Transfer zwischen Hochschule und gesellschaftlichen Interessen,

- das Marketing für wissenschaftliche Resultate und Methoden.

Das Leistungsspektrum einer ausgebauten Einrichtung für Weiterbildung umfasst dann bei der Entwicklung, Planung, Beratung und Auswertung von Angeboten wissenschaftlicher Weiterbildung folgende Aufgaben:

- Bedarfsanalysen,

- Programmplanung,

- didaktische Beratung,

- Methodenberatung,

- Medienbereitstellung,

- Qualitätssicherung,

- Dozent/innen-Vermittlung,

- Kursentwicklung,

- Kontaktherstellung,

- Lernberatung, 
- Personalberatung,

- Finanzierungsberatung,

- Forschungsrecherchen.

Wissenschaftliche Weiterbildung ist keine einfache Brücke, welche die „Lücke“ zwischen Praxis und Wissenschaft elegant schließen könnte. Sie ist vielmehr ein voraussetzungsreicher und kontinuierlicher Prozess der sorgfältigen Analyse weiterbildungsrelevanter Entwicklungen in Praxis und Wissenschaft und deren Vermittlung $\mathrm{zu}$ forschungsgeleiteter Wissensproduktion. Wissenschaftliche Weiterbildung entsteht in Netzwerken und kooperativen Arbeitszusammenhängen, die in aller Regel erst einmal aufgebaut werden müssen.

Einrichtungen für Weiterbildung an Hochschulen mit einem solchen Profil sind intermediäre Institutionen. Sie sind dies nicht per Definition, sondern durch ihr Handeln und ihre intermediäre Vermittlung zwischen den sachlichen Ebenen des Bedarfs, der Gestaltung und des Transfers von Wissen als intermediärem Produkt und den persönlichen Ebenen der beteiligten Akteure. Ihre „Partner“ im engeren Sinne sind Wissenschaftler/innen als Wissensproduzenten und Lehrende und die Lernenden als "Suchende“ und Anwender neuen Wissens.

In vielen Fällen übernimmt die für die Weiterbildung einer Hochschule zuständige Stelle auch noch weitere Aufgaben, die mit der Weiterbildung verwandt sind (oder auch nicht!). Für die Profilierung ist es ungünstig, wenn das Aufgabenspektrum zu breit oder der Zusammenhang zwischen den Aufgaben gering ist. Andererseits ermöglicht die Zusammenfassung verwandter Aufgaben an einer Stelle eine bessere Abstimmung und die Nutzung von Synergien.

- Aufgabenfelder von Einrichtungen für das LLL: Weiterbildung, Wissenstransfer, Fernstudium, Seniorenstudium und -universität, Hochschuldidaktik, überfachliche Kompetenzen, offene Hochschule, Fortbildung für Lehrerinnen und Lehrer, Alumniorganisation, Tag der Forschung, öffentliche Wissenschaft, Medien- und E-Learning-Zentrum, Beratung und Service, Forschung und Dienstleistungen wie Kongressmanagement usw.

\section{Empfehlungen}

Es gibt keine Musterlösung für die richtige Organisationsform. Insbesondere abhängig vom Aufgabenspektrum, den Organisationszielen und der bestehenden Organisation und Kultur der Hochschule ist individuell die optimale Lösung zu suchen.

Die DGWF empfiehlt dabei vor allem Folgendes zu beachten:

- Die Aufgabenbreite ist so festzulegen, dass eine Profilierung der Einrichtung möglich ist.

Für ein erfolgreiches Bildungsmanagement ist eine Bündelung aller Aufgaben und Funktionen im Kontext der wissenschaftlichen Weiterbildung in einer Einrichtung sinnvoll. Die damit verbundene Profilierung der Einrichtung nach in- 
nen und außen verdeutlicht die Intention der Hochschule, die wissenschaftliche Weiterbildung konsequent auszubauen.

- Für eine hohe Reputation ist der wissenschaftliche Bezug von großer Bedeutung.

Wissenschaftliche Weiterbildung zeichnet sich inhaltlich durch ihren Forschungsbezug aus. Bedarfserhebung, Konzeptentwicklung und Qualitätssicherung erfordern eigenständige wissenschaftliche Tätigkeiten. Dies spricht für eine intermediäre und/oder wissenschaftlich ausgerichtete Verortung.

- Ein explizites Commitment der Hochschulleitung stärkt die wissenschaftliche Weiterbildung.

Die wissenschaftliche Weiterbildung ist Teil des Lehr- und Bildungsauftrages der Hochschule. Die Hochschulleitung fördert aktiv die inhaltliche und organisatorische Entwicklung dieses Bereiches. Wissenschaftliche Weiterbildung ist Teil des Leitbildes der Hochschule und des Hochschulentwicklungsplanes.

- Für die Sichtbarkeit und die Durchsetzungskraft zugunsten der Weiterbildung ist eine Einrichtung von Vorteil, die zumindest einen wichtigen Teil der Funktionen zentral erfüllt.

Die zentrale Ansiedlung der Einrichtung im third space verdeutlicht ihren Stellenwert für die gesamte Hochschule und hebt den intermediären Charakter ihrer Aufgaben und Funktionen hervor.

- Auslagerungen können in Abhängigkeit von der spezifischen Standortsituation und den Aufgaben der Einrichtung eine sinnvolle Lösung sein.

Die wissenschaftliche Weiterbildung ist originäre Aufgabe der Hochschulen. Die Umsetzung dieses Auftrages erfolgt in enger Kooperation mit dem Wissenschaftsbereich und der Hochschulverwaltung. Dies ist bei einer Auslagerung sicherzustellen, damit die Identifikation der Hochschule mit der Einrichtung für Weiterbildung nicht beeinträchtigt wird.

Im Weiteren zeigen die Erfahrungen, dass sich die folgenden Bedingungen für eine erfolgreiche wissenschaftliche Weiterbildung an Hochschulen besonders günstig auswirken:

- klare Aufgabenbeschreibungen, Verantwortlichkeiten und Kompetenzen für die Akteure der Weiterbildung,

- mittel- bis längerfristig gefestigte Lösungen bezüglich Organisationsform, Aufgaben und Kompetenzen,

- angemessene und langfristig gesicherte personelle und finanzielle Mittel sowie Infrastruktur,

- Absicherung des finanziellen Risikos für Entwicklung und Ausfall von Angeboten,

- Handlungsspielräume für marktwirtschaftliches Verhalten,

- direkter Zugang der Weiterbildungsverantwortlichen zu den Lehrenden und Forschenden, Möglichkeit für Anreize zur Lehre in der Weiterbildung,

- enger Bezug zu den Fakultäten, Anerkennung der Weiterbildung in der Academia. 


\section{Literatur}

DGWF Hochschule und Weiterbildung (2/2013): Schwerpunktthema: Re-Organisation wissenschaftlicher Weiterbildung.

Dollhausen, K./Ludwig, J./Wolter, A. (2013): Organisation und Re-Organisation wissenschaftlicher Weiterbildung in einer bewegten Hochschullandschaft. In: Hochschule und Weiterbildung 2/2013, DGWF Bielefeld.

EU-Kommission (2001): Einen europäischen Raum des lebenslangen Lernens schaffen, Brüssel: URL: http://www.bibb.de/dokumente/pdf/foko6_neues-aus-euopa_o4_raum1ll.pdf (letzter Zugriff 20.07.2015).

Faulstich, P./Graeßner, G./Bade-Becker, U./Gorys, B. (2007): Länderstudie Deutschland. In: Hanft, A./Knust, M. a. a. O., S. 84-188.

Hanft, A./Knust, M. (2007): Internationale Vergleichsstudie zur Struktur und Organisation der Weiterbildung an Hochschulen, Oldenburg.

Kottmann, A./Nickel, S. (2010): Wissenschaftsmanagement - Terra incognita der Wissenschaft? Überblick über den Forschungsstand. In: Nickel, S./Ziegele, F. a. a. O., S. 24.

Nickel, S./Ziegele, F. (2010): Karriereförderung im Wissenschaftsmanagement - nationale und internationale Modelle. CHE Gütersloh 2010 (https://www.bmbf.de/pubRD/ O_CHE-STUDIE_Endbericht_final.pdf, letzter Zugriff 20.07.2015).

Whitchurch, C. (2004): Administrative Managers - A Critical Link. In: Higher Education Quarterly. Vol. 58, No. 4, S. 280-298. 



\section{Formate wissenschaftlicher Weiterbildung}

\section{DGWF-Empfehlung, beschlossen im Dezember 2010}

\section{Selbstverständnis wissenschaftlicher Weiterbildung und Ausgangslage}

Wissenschaftliche Weiterbildung findet als eine der Kernaufgaben der Hochschulen ihren Platz neben der Forschung und wird im Zusammenwirken mit dem grundständigen Studium zu einem selbstverständlichen Teil der akademischen Lehre (Graeßner et al. 2009, S. 543). Sie umfasst berufliche, politische und allgemeine Bildung in einer Vielzahl von Veranstaltungsformen, von Einzelveranstaltungen über systematisierte Programme bis hin zu mehrsemestrigen weiterbildenden Studiengängen.

Wissenschaftliche Weiterbildungsangebote der Hochschulen wenden sich an Personen mit einem ersten Hochschulabschluss und an Personen, die sich beruflich oder auf andere Weise für eine Teilnahme qualifiziert haben und ermöglichen organisierte, zielgruppengerechte Lernprozesse auf fachlichem und didaktisch-methodischem Niveau der Hochschule. Ihre enge inhaltliche Anbindung an die Forschung sowie ihre Doppelfunktion - mit ihr wird einerseits eine Dienstleistung für die Gesellschaft erbracht, andererseits verhält sie sich ihr gegenüber zugleich kritisch distanzierend, indem sie die Praxis mit ihren eigenen Fragestellungen und Erkenntnissen konfrontiert - zeichnet wissenschaftliche Weiterbildung an Hochschulen im Besonderen aus (vgl. Dikau 1999, S.13 f.).

Die Studienorganisation und die damit verbundene Vereinbarkeit der Angebote mit Familie und Beruf entscheiden wesentlich über die Teilnahme der Adressaten. Die in Präsenzform, als Fernstudien oder in Blended Learning-Form vorgehaltenen Angebote orientieren sich daher grundsätzlich an den zeitlichen und organisatorischen Rahmenbedingungen der Adressaten (Faulstich et al. 2007, S. 132 f.).

Mit der vorgefundenen Angebotsvielfalt, die es zu erhalten gilt, wird jeweils auf spezifische Nachfragen reagiert (DGWF 2005, S. 8). 
Bei der Entscheidung für eine Weiterbildung kann für die Lernenden einerseits die inhaltliche Bedeutsamkeit der Themen für individuelle Verwendungszusammenhänge leitend sein und ein formaler Abschluss nicht zwingend im vorrangigen Interesse stehen.

Andererseits gewinnt die persönliche Verwertbarkeit von Abschlüssen und Zertifikaten - insbesondere im beruflichen Kontext - zunehmend an Bedeutung.

Im Berliner Kommunique der Bologna-Nachfolge-Konferenz der europäischen Hochschulministerinnen und -minister (2003, S. 5) wird die bedeutende Rolle des European Credit Transfer System (ECTS) für die Förderung der studentischen Mobilität und die internationale Curriculumentwicklung hervorgehoben. Das ECTS soll nicht nur die allgemeine Grundlage für nationale Leistungspunktsysteme stellen, sondern gleichfalls zu einem System für die Übertragbarkeit und Kumulierung von Leistungspunkten führen, das im gesamten europäischen Hochschulraum einheitliche Anwendung findet - ergo auch für das Segment der wissenschaftlichen Weiterbildung.

Die nicht einheitliche Bezeichnung der „Formate“ unterhalb der Ebene „weiterbildender Masterstudiengang“ in den Bundesländern - ein Sachverhalt, der den jeweiligen Traditionen und der entsprechenden Terminologie in den Hochschulgesetzen ${ }^{1}$ geschuldet ist - steigert die Intransparenz der wissenschaftlichen Weiterbildungsangebote auf einem zunehmend internationaler werdenden wettbewerblichen Markt.

\section{Systematisierung und Empfehlungen}

Durch die Reorganisation der Studienstruktur im BA/MA-System wird der Stellenwert von Zertifikaten in der Weiterbildung neu bestimmt. Dabei tauchen eine Reihe von Fragen auf:

- Soll es weiterbildende Bachelor-Studiengänge geben?

- Sind Masterstudiengänge insgesamt als Weiterbildung zu behandeln?

- Welchen Umfang sollen weiterbildende Masterstudiengänge haben?

- Wie können im Studium - als Lernen in der Hochschule - vorgängige, in der Berufsausbildung oder auf andere Art erworbene Kompetenzen angerechnet werden?

1 In den Hochschulgesetzen der Länder finden sich im Wesentlichen folgende Begriffe:

- weiterbildender Studiengang,

- weiterbildendes Studium,

- Kontaktstudium,

- Zusatzstudium,

- Ergänzungsstudium,

- Aufbaustudium.

Kontakt-, Zusatz- und Aufbaustudium sind im Sinne der HRK als „postgraduale Studien“ zusammenzufassen und im strengen Sinne der Weiterbildung nicht zuzuordnen. Mit der Einführung der gestuften Studiengänge werden diese Formate voraussichtlich obsolet (Faulstich et al. 2007, S. 96). 
- Wie werden Weiterbildungsanteile in die konsekutive BA-/MA-Struktur einbezogen?

- Wie können Übergangsmöglichkeiten zwischen Beruf, Berufsausbildung, Bachelor und Master, sowie zu gleichzeitiger oder anschließender Berufstätigkeit gestaltet und gesichert werden?

Mit der vorliegenden Empfehlung wird die Intention verfolgt, die Transparenz und die Vergleichbarkeit der wissenschaftlichen Weiterbildungsangebote sowie die Durchlässigkeit zwischen einzelnen Angeboten über eine stärkere Systematisierung und Standardisierung der Weiterbildungsformate und ihrer Abschlüsse inklusive einer einheitlichen Terminologie zu fördern. Es sollen zum einen maximale Übergangs- und Durchlässigkeitsmöglichkeiten geschaffen, zum anderen Anerkennung, Anrechnung und Verbindlichkeit erhöht und angestrebt werden. Folgende Effekte werden erwartet:

- die Ermöglichung der systematischen Verknüpfung der Angebote wissenschaftlicher Weiterbildung einzelner Hochschulen zu den grundständigen Studienangeboten und zu den Angeboten der beruflichen Weiterbildung,

- die Verdeutlichung der inhaltlichen und formalen Wertigkeit der Abschlüsse (über Leistungspunkte und Abschlussbezeichnungen),

- die Schärfung des Profils wissenschaftlicher Weiterbildungsangebote der Hochschulen.

Die Weiterbildungsaktivitäten der Hochschulen umfassen ein breites Spektrum: Es reicht von Angeboten, die einen Bachelor- oder Master-Abschluss voraussetzen, über eigenständige zertifizierte Weiterbildungsstudien bis hin zu Einzelveranstaltungen.

Folgende Systematisierungsstufen werden empfohlen:

1. Weiterbildende Masterstudiengänge

2. Weiterbildende bzw. berufsbegleitende Bachelorstudiengänge

3. Weiterbildende Zertifikatsstudien

4. Weiterbildungsmodule

5. Weiterbildungsseminare

6. Sonstige Weiterbildungsformate

In diesem Spektrum gilt ein besonderes Augenmerk der Gestaltung der „Weiterbildenden Master-Studiengänge“. Es kommt darauf an, eine Engführung zu verhindern und die Entwicklung offenzuhalten.

\subsection{Weiterbildende Masterstudiengänge}

Die KMK differenziert im Bereich der Masterstudiengänge in konsekutive und weiterbildende Masterstudiengänge. Die Rahmenanforderungen an weiterbildende Masterstudiengänge sind in den Ländergemeinsamen Strukturvorgaben für die Akkreditierung von Bachelor- und Masterstudiengängen (Kultusministerkonferenz 2010) geregelt. 
Der Zugang zu weiterbildenden Masterstudiengängen erfordert grundsätzlich neben einem berufsqualifizierenden Hochschulabschluss auch die qualifizierte berufspraktische Erfahrung von i.d. R. nicht unter einem Jahr. In Ausnahmefällen können die Landeshochschulgesetze vorsehen, dass an die Stelle des berufsqualifizierenden Hochschulabschlusses eine Eingangsprüfung treten kann (KMK 2010, S. 4).

Die Inhalte des weiterbildenden Masterstudiengangs berücksichtigen die beruflichen Erfahrungen der Weiterbildungsstudierenden und knüpfen an diese an.

Für den Masterabschluss werden unter Einbeziehung des vorangegangenen Studiums bis zum ersten berufsqualifizierenden Abschluss i. d. R. 300 ECTS-Punkte benötigt. Davon kann bei entsprechender Qualifikation der Studierenden im Einzelfall abgewichen werden. Das gilt auch dann, wenn nach Abschluss eines Masterstudiengangs 300 Leistungspunkte nicht erreicht werden (KMK 2010, S. 3).

Der quantitative Umfang des weiterbildenden Masterstudiengangs beträgt i. d. R. 6o bis 120 Leistungspunkte nach ECTS (vgl. KMK 2005, S.2). Außerhalb des Hochschulwesens erworbene Kenntnisse und Fähigkeiten können bis zu 50\% eines Hochschulstudiums ersetzen, wenn sie nach Inhalt und Niveau dem Teil des Studiums gleichwertig sind, der substituiert werden soll (KMK 2002, S. 2; KMK 2010, S.3). Für einen erfolgreich abgeschlossenen weiterbildenden Masterstudiengang wird der Mastergrad verliehen.

Empfehlung:

Der weiterbildende Masterstudiengang ist vom Umfang her ein sehr anspruchsvolles Format. Es wird empfohlen, Angebote dieser Art in Schwerpunkte zu gliedern, die formal jeweils ein „Weiterbildendes Zertifikatsstudium“ ergeben. Kumulativ kann darüber der Masterabschluss erreicht werden. Die nach den KMKBeschlüssen und den rechtlichen Regelungen der Länder mögliche Gestaltung des Zugangs, der Anrechnung von Qualifikationen auf fehlende Kreditpunkte sowie die Anerkennung vorgängiger Leistungen auf das Studium sollen extensiv genutzt werden.

\subsection{Weiterbildende bzw. berufsbegleitende Bachelorstudiengänge}

Obgleich im Rahmen individueller Bildungsentscheidungen auch konsekutive Masterstudiengänge und ebenso (berufsbegleitende) Bachelorstudiengänge als Weiterbildung genutzt werden, zählen diese bildungs- bzw. ordnungspolitisch bisher nicht zum Bereich der wissenschaftlichen Weiterbildung (vgl. Akkreditierungsrat 2007, S. 5).

Mit der verstärkten Einführung von berufsbegleitenden Bachelorstudiengängen sollen insbesondere qualifizierte Berufstätige für ein Hochschulstudium gewonnen werden. Gemäß der Strukturvorgaben der KMK (2010, S.3) sind für den Bachelorabschluss nicht weniger als 180 Leistungspunkte nach ECTS nachzuweisen, wobei gleichwertige Kompetenzen und Fähigkeiten, die außerhalb des Hochschulbereichs 
erworben wurden, bis zur Hälfte der für den Studiengang vorgesehenen Leistungspunkte angerechnet werden können.

Empfehlung:

Der weiterbildende resp. berufsbegleitende Bachelorstudiengang ist wie der weiterbildende Masterstudiengang ein sehr anspruchsvolles Format. Er sollte nicht nur organisatorisch ein berufsbegleitendes Studieren ermöglichen, sondern auch inhaltlich die beruflichen Erfahrungen der Weiterbildungsstudierenden aufnehmen und an diese anknüpfen.

\subsection{Weiterbildende Zertifikatsstudien: längerfristige Weiterbildungsangebote mit einem Arbeitspensum von zwölf bis 60 Leistungspunkten}

Unterhalb der weiterbildenden Studiengänge finden sich begrifflich insbesondere das Format des „weiterbildenden Studiums“ sowie des „Kontaktstudiums“. Diese Formate bezeichnen längerfristige Weiterbildungsangebote, die mit unterschiedlichen Abschlussmöglichkeiten ausgestattet sind (bspw. Zertifikate, Zertifikate und (nicht-)akademische Titelverleihung, Teilnahmeurkunde, Teilnahmebescheinigung).

Die vorgenannten Weiterbildungsformate sind zum Teil in den Hochschulgesetzen der Länder aufgeführt und mit Kriterien versehen.

Empfehlung zur Benennung und zu Mindestanforderungen:

\begin{tabular}{|l|l|}
\hline $\begin{array}{l}\text { Bezeichnung des } \\
\text { Angebotsformats }\end{array}$ & Weiterbildendes Zertifikatsstudium \\
\hline Zugang/Zulassung & Zielgruppenorientiert, mind. ein Jahr Berufstätigkeit \\
\hline Regelung & Zulassungs-, Studien- und Prüfungsregelung der Hochschule \\
\hline Umfang & zwölf bis 60 Leistungspunkte \\
\hline Struktur & $\begin{array}{l}\text { Curricular festgelegtes, modularisiertes Programm; Modulgröße i. d. R. je- } \\
\text { weils mindestens fünf LP oder ein Vielfaches davon }\end{array}$ \\
\hline Abschluss & $\begin{array}{l}\text { Zertifikat mit diploma supplement, Vergabe von Leistungspunkten und ge- } \\
\text { gebenenfalls eines Titels }\end{array}$ \\
\hline Durchlässigkeit & Module in affinem (weiterbildenden) Masterstudiengang anerkannt \\
\hline Qualitätssicherung & $\begin{array}{l}\text { Evaluation/en; (institutionsspezifisches) Qualitätsmanagementsystem; } \\
\text { ggf. Systemakkreditierung }\end{array}$ \\
\hline
\end{tabular}

Das „Weiterbildende Zertifikatsstudium“ ist ein lang erprobtes Format der wissenschaftlichen Weiterbildung, das zunehmend Akzeptanz auf der Abnehmerseite gewinnt; seine behutsame Standardisierung erhöht die Attraktivität und eröffnet formale Möglichkeiten der Durchlässigkeit und Kombination mit affinen weiterbildenden Masterstudiengängen oder anderen weiterbildenden Zertifikatsstudien.

2 Zur Modulgröße vgl. KMK 2010. 


\subsection{Weiterbildungsmodule: Module eines akkreditierten Studiengangs als Weiterbildungsangebot}

\section{Empfehlung zur Benennung und zu Mindestanforderungen:}

\begin{tabular}{|l|l|}
\hline $\begin{array}{l}\text { Bezeichnung des } \\
\text { Angebotsformats }\end{array}$ & $\begin{array}{l}\text { Weiterbildungsmodul } \\
\text { (i. d. R. Modul eines akkreditierten weiterbildenden } \\
\text { Studiengangs) }\end{array}$ \\
\hline Zugang/Zulassung & offen für alle Interessierten, die den inhaltlichen Anforderungen entsprechen \\
\hline Regelung & Modulhandbuch der Hochschule \\
\hline Umfang & i.d. R. mindestens 5 LP oder ein Vielfaches davon \\
\hline Struktur & Curricular festgelegter Abschnitt (Modul) eines Studiengangs \\
\hline Abschluss & $\begin{array}{l}\text { Modulprüfungszeugnis mit Notenangabe und Angabe der erworbenen Leistungs- } \\
\text { punkte }\end{array}$ \\
\hline Durchlässigkeit & Modul in affinem Bachelor- bzw. (weiterbildenden) Masterstudiengang anerkannt \\
\hline Qualitätssicherung & $\begin{array}{l}\text { Evaluation/en; (institutionsspezifisches) Qualitätsmanagementsystem; ggf. System- } \\
\text { akkreditierung }\end{array}$ \\
\hline
\end{tabular}

\subsection{Weiterbildungsseminare}

\section{Empfehlung zur Benennung und zu Mindestanforderungen:}

\begin{tabular}{|l|l|}
\hline $\begin{array}{l}\text { Bezeichnung des } \\
\text { Angebotsformats }\end{array}$ & Weiterbildungsseminar \\
\hline Zugang/Zulassung & offen für alle Interessierten, die den inhaltlichen Anforderungen entsprechen \\
\hline Regelung & nicht definiert; optional: Rahmenordnung der Hochschule \\
\hline Umfang & i. d. R. drei bis fünf LP \\
\hline Struktur & ggf. curricular festgelegte Veranstaltung \\
\hline Abschluss & qualifizierte Teilnahmebescheinigung und Vergabe von LP \\
\hline Durchlässigkeit & Möglichkeit der Anerkennung von Leistungen, sofern LP vergeben wurden. \\
\hline Qualitätssicherung & $\begin{array}{l}\text { Evaluation/en; (institutionsspezifisches) Qualitätsmanagementsystem; ggf. System- } \\
\text { akkreditierung }\end{array}$ \\
\hline
\end{tabular}

3 Bei den Modulen handelt es sich zwar um Module akkreditierter weiterbildender Masterstudiengänge, welche sich an Personen mit erstem Hochschulabschluss wenden. Dieses schließt jedoch nicht aus, den Zugang zu Modulen auch anderen interessierten Personenkreisen zu ermöglichen, die nicht diesen Anforderungen entsprechen.

4 Bei den Modulen handelt es sich zwar um Module akkreditierter weiterbildender Masterstudiengänge, welche sich an Personen mit erstem Hochschulabschluss wenden. Dieses schließt jedoch nicht aus, den Zugang zu Modulen auch anderen interessierten Personenkreisen zu ermöglichen, die nicht diesen Anforderungen entsprechen. 


\subsection{Sonstige Weiterbildungsformate}

Die Vielfalt der Veranstaltungsformate im Bereich wissenschaftlicher Weiterbildung gilt es zu erhalten, denn sie entspricht den Bedürfnissen aufseiten der Nachfrage.

$\mathrm{Zu}$ den Veranstaltungsformaten, die nicht den unter 2.1 bis 2.5 genannten Formaten ${ }^{5}$ entsprechen, wird folgende Empfehlung gegeben:

\begin{tabular}{|l|l|}
\hline $\begin{array}{l}\text { Bezeichnung des } \\
\text { Angebotsformats }\end{array}$ & nicht definiert; Kategorie: sonstige Weiterbildungsangebote \\
\hline Zugang/Zulassung & $\begin{array}{l}\text { offen für alle Interessierten, die den inhaltlichen } \\
\text { Anforderungen entsprechen }\end{array}$ \\
\hline Regelung & nicht definiert; optional: Rahmenordnung der Hochschule \\
\hline Umfang & i. d. R. bis drei LP \\
\hline Struktur & ggf. curricular festgelegte Veranstaltung \\
\hline Abschluss & $\begin{array}{l}\text { - Teilnahmebescheinigung ohne Vergabe von LP oder } \\
\text { - qualifizierte Teilnahmebescheinigung und Vergabe von LP }\end{array}$ \\
\hline Durchlässigkeit & Möglichkeit der Anerkennung von Leistungen, sofern LP vergeben wurden \\
\hline Qualitätssicherung & $\begin{array}{l}\text { Evaluation/en; (institutionsspezifisches) Qualitätsmanagementsystem; ggf. System- } \\
\text { akkreditierung }\end{array}$ \\
\hline
\end{tabular}

\section{Verknüpfungen und Kombinationsmöglichkeiten der Weiterbildungsformate}

Mit den vorgenannten Empfehlungen soll die Vielfalt der vorhandenen Formate wissenschaftlicher Weiterbildung an den Hochschulen nicht eingeschränkt werden. Sie folgen den Prinzipien des Adressatenbezugs und der Teilnehmerorientierung, z. B. bei besonderen Angeboten für Ältere. Sie wollen deshalb die Nutzbarkeit der Formate untereinander bzw. füreinander aufzeigen und fördern und damit Vergleichbarkeit und Durchlässigkeit ermöglichen. Dieses setzt einen gewissen Grad an Systematisierung voraus. So ist denkbar, dass die Kumulierung „kleinerer“ Formate zum Abschluss eines Zertifikatsstudiums führen bzw. innerhalb dessen als bereits erbrachte Leistung/en anerkannt werden kann. Zertifikatsstudien wiederum können als Bestandteil weiterbildender Masterstudiengänge ihrerseits als erbrachte Studienleistungen anerkannt werden. Eine maßvolle Systematisierung und Standardisierung fördert die horizontale und vertikale Durchlässigkeit innerhalb und zwischen den einzelnen Formaten der wissenschaftlichen Weiterbildung und zu den grundständigen Studienangeboten.

5 Z. B. Tagung, Kongress, Kurs, Workshop, Inhouse-Schulung. 


\section{Literatur}

Akkreditierungsrat (2007): Handreichung des Akkreditierungsrates an die Agenturen auf Grundlage der „Empfehlungen der Arbeitsgruppe ,Weiterbildende Studiengänge' des Akkreditierungsrates zur Qualitätssicherung und Akkreditierung weiterbildender Masterstudiengänge“. http://www.akkreditierungsrat.de/fileadmin/Seiteninhalte/Be schluesse_AR/2007_10_14_Empfehlungen_der_AG_Weiterbildung-Handreichung_ Agenturen_neu.pdf

DGWF-Empfehlung (2005): Perspektiven wissenschaftlicher Weiterbildung in Deutschland aus Sicht der Einrichtungen an Hochschulen. Beschlossen am 17.9.2005 in Wien.

Dikau, J. (1999): Zwischen öffentlicher Verantwortung und Marktorientierung. In: AUEInformationsdienst Hochschule und Weiterbildung 2/1999, Jena 1999, S.10-23.

Faulstich, P./Graeßner, G./Gorys, B./Bade-Becker, U. (2007): Länderstudie Deutschland. In: Hanft, A./Knust, M. (Hrsg.): Internationale Vergleichsstudie zur Struktur und Organisation der Weiterbildung an Hochschulen, S. 84-188. http://www.bmbf.de/pub/ internat_vergleichsstudie_struktur_und_organisation_hochschulweiterbildung.pdf

Graeßner, G./Bade-Becker, U./Gorys, B. (2009): Weiterbildung an Hochschulen. In: Tippel, R./von Hippel, A. (Hrsg.): Handbuch Erwachsenenbildung/Weiterbildung. 3., überarbeitete und erweiterte Auflage, Wiesbaden 2009, S. 543-555.

Kommunique der Konferenz der europäischen Hochschulministerinnen und -minister am 19. September 2003 in Berlin (2003): „Den Europäischen Hochschulraum verwirklichen“. http://www.hrk-bologna.de/bologna/de/download/dateien/Berliner_Kommu nique.pdf

Kultusministerkonferenz (2002): Anrechnung von außerhalb des Hochschulwesens erworbenen Kenntnissen und Fähigkeiten auf ein Hochschulstudium (I). Beschluss der Kultusministerkonferenz vom 28.06.2002. http://www.akkreditierungsrat.de/filead min/Seiteninhalte/Dokumente/kmk/KMK_AnrechnungausserhalbHochschule_l.pdf

Kultusministerkonferenz (2005): Qualifikationsrahmen für Deutsche Hochschulabschlüsse. Beschluss der Kultusministerkonferenz vom 21.04.2005; im Zusammenwirken von Hochschulrektorenkonferenz, Kultusministerkonferenz und Bundesministerium für Bildung und Forschung erarbeitet. http://www.akkreditierungsrat.de/ fileadmin/Seiteninhalte/Dokumente/kmk/KMK_050421_Qualifikationsrahmen.pdf

Kultusministerkonferenz (2010): Ländergemeinsame Strukturvorgaben für die Akkreditierung von Bachelor- und Masterstudiengängen. Beschluss der Kultusministerkonferenz vom 10.10.2003 i.d. F. vom 04.02.2010. http://www.kmk.org/fileadmin/veroeffentlich ungen_beschluesse/2003/2003_10_10-Laendergemeinsame-Strukturvorgaben.pdf 


\section{Perspektiven wissenschaftlicher Weiterbildung in Deutschland aus Sicht der Einrichtungen an Hochschulen}

DGWF-Empfehlung, beschlossen am 17.09.2005 in Wien

\section{Vorbemerkung}

Immer dringlicher wird die Frage nach der Zukunft wissenschaftlicher Weiterbildung in dem sich entwickelnden Hochschulraum Europa gestellt. Durch die Schaffung einheitlicher Grundstrukturen der Hochschulsysteme hat die wissenschaftliche Weiterbildung starken Aufwind erhalten. Sie tritt aus einer Randrolle ins Zentrum der Diskussion um die Perspektiven von Lehre. Stichwörter dafür sind die neu zu organisierenden Bachelor- und Master-Studiengänge; diese erhöhen die Flexibilität und die Verknüpfungen zwischen grundständigen und weiterführenden Studien. Gleichzeitig entsteht daraus ein unübersichtliches Feld, in dem die Diversität der Lernmöglichkeiten wächst. Dies begründet für die „Deutsche Gesellschaft für wissenschaftliche Weiterbildung und Fernstudium“ die Notwendigkeit einer koordinierten Strategie der wissenschaftlichen Weiterbildung für Europa.

Durch den Bologna-Prozess ist nun vieles in Bewegung gekommen. Dabei gibt es widersprüchliche Einschätzungen:

1. Der Bologna-Prozess ist für die wissenschaftliche Weiterbildung eine große Chance. Durch die Reorganisation der Studienstrukturen entstehen unverhoffte Möglichkeiten, Verknüpfungen zwischen Erstausbildung und Weiterbildung herzustellen. Spätestens im Zusammenhang der Diskussion um die MasterProgramme ist eine Trennung kaum noch systematisch begründbar. Die Rückkehr an die Hochschulen zur Weiterbildung nach einer zwischenzeitlichen Berufstätigkeit wird selbstverständlich. Viele Hochschulen befinden sich mittlerweile im Prozess einer konsequenten „Bolognarisierung“. 
2. Der Bologna-Prozess ist ein großes Risiko. Gerade wenn man an die Tradition der abendländischen Universität denkt, war die Freiheit von Studium und Lehre einer ihrer wichtigsten Grundpfeiler. Diesem droht nun durch die Standardisierung der Studienorganisation eine Tendenz zur Verschulung. Modularisierung erschwert die in der Weiterbildung notwendige Flexibilisierung der Angebote. Strikte Beschäftigungsfähigkeit mündet eher in einer Überforderung der Hochschulangebote. Die Risiken werden verstärkt, weil gleichzeitig nicht nur der Bologna-Prozess, sondern mindestens noch die Reorganisation durch neue Managementstrukturen und außerdem die Forderung nach Exzellenz und Elite parallel laufen.

3. Der Bologna-Prozess ist eine Herausforderung. Es geht nicht darum, sich zurückzulehnen und die Entwicklung treiben zu lassen. Im Rahmen der widersprüchlichen Tendenzen gibt es auch Gestaltungsstrategien, welche die Vielfalt von Diversität und Systematik strukturieren müssen. Hier könnte eine „mittlere Systematisierung“ greifen, welche zwischen Marktregulation und Autonomieansprüchen vermittelt.

Die Erfahrung zeigt, dass wissenschaftliche Weiterbildung an Hochschulen dann Kontinuität erhält, wenn intermediäre Institutionen zwischen Fachbereichen und Fakultäten einerseits und Präsidial- bzw. Rektoratsverwaltungen andererseits geschaffen werden, welche übergreifende Aktivitäten organisieren: Bedarfsanalysen, Programmplanung, didaktische Beratung, Methodenberatung, Medienbereitstellung, Qualitätssicherung, Dozentenvermittlung, Unterstützung bei der Kursentwicklung, Unterstützung bei der Entwicklung von E-Learning-Konzepten, Kontaktherstellung, Lernberatung, Personalberatung, wissenschaftliche Begleitung, Finanzierungsberatung und Forschungsrecherchen. Solche Einrichtungen wissenschaftlicher Weiterbildung befördern Initiativen, sichern die Kontinuität der Aktivitäten und sind Ansprechpartner für die wachsende Weiterbildungsnachfrage.

Die Breite möglicher Gestaltungsfelder führt zu hohen Anforderungen. Für die wissenschaftliche Weiterbildung müssen deshalb einige Strukturfragen geklärt werden: die Zugangsfrage, die Strukturproblematik, die Abschlussproblematik, die Integration von Aus- und Weiterbildung, das Verhältnis von Hochschulangeboten und externen Institutionen. Hochschulintern besteht Entwicklungsbedarf bezogen auf die Kapazitäts- und die Deputatswirksamkeit wissenschaftlicher Weiterbildung, die Qualitätssicherung der Angebote, ihre Kosten und Finanzierung, die fachbereichsübergreifende Organisation und die Stärkung eines eigenen wissenschaftlichen Profils.

Mindestens zu diesen Fragen ist eine koordinierte Strategie sinnvoll, welche für die Adressaten der Weiterbildungsangebote Transparenz und Auswahlsicherheit schafft. Die folgenden Empfehlungen richten sich an hochschul- und weiterbildungspolitische Akteure innerhalb und außerhalb der Hochschulen. Sie konzentrieren sich auf Organisations- und Ressourcenfragen, die entschieden werden müssen, um dem Expansionsprozess wissenschaftlicher Weiterbildung Kontinuität zu geben und gleichzeitig neue Initiativen zu entfalten. 


\section{Ausgangslage und Selbstverständnis wissenschaftlicher Weiterbildung}

Wissenschaftliche Weiterbildung ist Kernaufgabe der Hochschulen in Deutschland. Sie ist Strukturprinzip von Lehre und des Transfers von Forschung. Die Diskussion über wissenschaftliche Weiterbildung hat nicht nur im hochschulpolitischen Umfeld Aufwind.

Bereits jetzt hat dieses Aktivitätsspektrum erhebliches Gewicht: Im „Hochschulkompass“ der Hochschulrektorenkonferenz findet man über 1.500 Einträge in der Rubrik „Weiterführende Studienangebote“. (Im Vergleich dazu sind etwa 9.000 grundständige Studienmöglichkeiten verzeichnet.) Es werden außerdem mehr als 300 Weiterbildungsinstitutionen im Hochschulkontext ausgewiesen. Eine Recherche der „Deutschen Gesellschaft für wissenschaftliche Weiterbildung und Fernstudium“ (DGWF) schätzt die jährlichen Teilnahmefälle auf fast 100.000. Zusätzlich weist die Gasthörerstatistik der Hochschulen etwa 40.000 Teilnehmende aus. Die Gesamtzahl dürfte noch wesentlich höher sein.

Der Bedarf an wissenschaftlicher Weiterbildung nimmt zu. So sieht sich Weiterbildung an Hochschulen neuen Herausforderungen im Rahmen „lebenslangen Lernens“ gegenüber. Sich verkürzende Wissenshalbwertszeiten bezogen auf fachliche Inhalte betreffen Hochqualifizierte besonders.

Ein gewichtiger Anteil wissenschaftlicher Weiterbildung wird an und durch Hochschulen realisiert. Nach wie vor liegt dabei der Schwerpunkt der Angebote unterhalb der Studiengänge mit akademischem Abschluss. Dies verschiebt sich zusehends.

Es finden sich seit mehr als 30 Jahren unterschiedlich weite und anspruchsvolle Fassungen für das Verständnis wissenschaftlicher Weiterbildung bei den verschiedenen einschlägigen Gremien. Die Umbruchsituation drängt dazu, die Organisation von Lehre, welche dann grundständige wie weiterführende Angebote einbezieht, insgesamt neu zu klären. In jüngerer Zeit haben vor allem zwei Stellungnahmen die wissenschaftliche Weiterbildung der Hochschulen wesentlich gefördert:

- der Sachstands- und Problembericht zur „Wahrnehmung wissenschaftlicher Weiterbildung an den Hochschulen“ der Kultusministerkonferenz (KMK) aus dem September 2001 und

- der 10-Punkte-Plan von HRK, BDA und DIHT vom Februar 2003.

Unter wissenschaftlicher Weiterbildung wird von der KMK „die Fortsetzung oder Wiederaufnahme organisierten Lernens nach Abschluss einer ersten Bildungsphase und in der Regel nach Aufnahme einer Erwerbs- oder Familientätigkeit” verstanden und dann auf Hochschulen bezogen, „wobei das wahrgenommene Weiterbildungsangebot dem fachlichen und didaktischen Niveau der Hochschule entspricht" (KMK 2001, 2).

Angemessene Reaktionen auf den wachsenden Bedarf an wissenschaftlicher Weiterbildung sollen erleichtert und ausgebaut werden durch: 
- den Abbau hinderlicher gesetzlicher Rahmenbedingungen (Haushalts-, Dienstund Besoldungsrecht),

- Schaffung zusätzlicher Anreize für die Hochschulen,

- die Liberalisierung des Hochschulzugangs,

- die weitere Gestaltung des Hochschuldienstrechts,

- regionale Netzwerke für wissenschaftliche Weiterbildung mit Hochschulen und Wirtschaft,

- Gründung bzw. Stärkung der „Einrichtungen für Weiterbildung“ an den einzelnen Hochschulen selbst,

- die Modularisierung der Studien- und Lernangebote, inkl. der - auch teilzeitstudiengeeigneten - Bachelor- und Masterstudiengänge.

In den Prozessen der Um- und Neustrukturierung der Hochschulen entwickeln sich Reorganisationstendenzen, wobei wissenschaftliche Weiterbildung stärker in das Zentrum der Hochschulen gerückt ist. Die Differenz zwischen grundständigem und weiterbildendem Studium wird durch die BA/MA-Diskussion aufgeweicht. Die Hochschulen sind insgesamt Prozessen der Um- und Neustrukturierung unterworfen. $\mathrm{Zu}$ nennen sind

- Veränderungen der Studienstruktur im Rahmen des Bologna-Prozesses (Bachelor- und Masterabschlüsse, Modularisierung),

- Akkreditierung der Studienangebote,

- Ökonomisierung, extern im Rahmen der Hochschulentwicklungsplanung von Ländern und Bund und hochschulintern im Rahmen von Managementmodellen, Profilbildungsprozessen und Exzellenzstrategien.

Die Organisationsentwicklungsprozesse verlaufen häufig nicht immer sach- und zielorientiert, da sich innerhalb vieler Hochschulen eher taktische und partikulare Interessen halten, beruhend auf der

- Selbsterhaltung von Institutionen,

- Entwicklung von Parallelstrukturen ohne ausreichende Abstimmung,

- Altersstruktur scheidenden Personals und entsprechende Rücksichtnahmen,

- geringen Aufmerksamkeit der Hochschulleitungen gegenüber wissenschaftlicher Weiterbildung.

Demgegenüber setzt sich die DGWF für einen kontinuierlichen, sach- und zielgerichteten Prozess der Entwicklung der wissenschaftlichen Weiterbildung in Deutschland an und in den Hochschulen ein. 


\section{Aufgaben der Einrichtungen für Weiterbildung an Hochschulen}

Mit der Diskussion um den Bologna-Prozess, in der einerseits der Stellenwert wissenschaftlicher Weiterbildung aufgewertet worden ist, andererseits und gleichzeitig die Rolle der Fakultäten bzw. Fachbereiche gestärkt wird, ist die Situation der zentralen Einrichtungen brisant geworden. Bisherige „Nischen“ lösen sich auf. Teilweise wird damit die Stabilität der Institutionen gefährdet; teilweise werden Ressourcen reduziert, Aufgaben ausgelagert und Personal abgebaut. Gleichzeitig gibt es eine „proklamatorische“ Aufwertung.

Diese Lage macht eine Neubestimmung der Stellung der Einrichtungen für wissenschaftliche Weiterbildung notwendig. Durch den Ausbau des Weiterbildungsbereiches haben sich die Gewichte im Verhältnis von Hochschulen und externen Kooperationspartnern deutlich verschoben. Weiterbildung ist zu einer eigenständigen und selbstverständlichen Aufgabe geworden.

Durch die interne Reorganisation des Wissenschaftsbereichs wird auch das Verhältnis der Fachbereiche zur Hochschulleitung und Verwaltung verändert. Durch die eigenständigeren Aktivitäten der Fakultäten werden einige zentrale Funktionen umverlagert bzw. verschwinden. Deshalb muss die intermediäre Institutionalisierung der zentralen Einrichtungen zwischen dezentralen Wissenschaftsaktivitäten und zentralen Leitungsfunktionen neu bestimmt werden. Zunächst geht es darum, ihre Aufgaben zu redefinieren.

Dabei muss unterschieden werden zwischen den Funktionen wissenschaftlicher Weiterbildung sowie den Aufgaben und der Organisation der einschlägigen Einrichtungen für Weiterbildung an Hochschulen. Es verschärft sich eine Paradoxie: Die Bedeutung wissenschaftlicher Weiterbildung nimmt zu und ist im hochschulpolitischen Diskurs unbestritten; das Gewicht der sie organisierenden Einrichtungen ist infrage gestellt und immer wieder neu zu bestimmen. Es gilt deshalb, wenn man die Einrichtungen für Weiterbildung an Hochschulen für wichtig und unverzichtbar hält, Aufgaben zu identifizieren, welche ohne Verluste an Funktionalität und Effizienz von der Hochschulverwaltung oder den Fachbereichen nicht ausgefüllt werden. Dazu muss ein prägnanter Leistungskatalog erstellt werden. Dieser sollte den Einrichtungen hochschulübergreifend gemeinsam sein, damit nach außen unmittelbar verständlich ist, was ihr Profil ausmacht.

Zentrale Aufgaben der Einrichtungen wissenschaftlicher Weiterbildung, welche von anderen Institutionalisierungsformen im Wissenschaftsbereich nicht erfüllt werden, beziehen sich hauptsächlich auf das „Bildungsmanagement“ wissenschaftlicher Weiterbildung. Dies ist nicht nur eine betriebswirtschaftliche, sondern vor allem eine bildungswissenschaftliche Aufgabe, die gefasst werden kann unter dem Begriff der Makrodidaktik, wenn es darum geht, die Rahmenbedingungen für das Lernen wissenschaftlichen Wissens zu organisieren. Es ist deutlich zu machen, dass dies nicht 
nur eine organisatorische oder administrative, sondern eine eigenständige wissenschaftliche Aufgabe ist, welche auch Forschungsaktivitäten impliziert.

Demgemäß erfüllen Einrichtungen für Weiterbildung für die Hochschulen eine Reihe von Funktionen:

- Entwicklung und Erschließung von Themen,

- Erschließung von Adressatinnen und Adressaten,

- Innovation der Organisation, Methoden und Problemperspektiven,

- Transfer zwischen Hochschule und gesellschaftlichen Interessen,

- Marketing für wissenschaftliche Resultate und Methoden,

- disziplinübergreifende Bearbeitung von Problemstellungen.

Das Leistungsspektrum eines ausgebauten Lernzentrums wissenschaftlicher Weiterbildung umfasst bei der Entwicklung, Planung, Beratung und Auswertung Aufgaben wie:

- Bedarfsanalysen,

- Programmplanung,

- Didaktische Beratung,

- Methodenberatung,

- Medienbereitstellung,

- Qualitätssicherung,

- Dozent/innenvermittlung,

- Kursentwicklung,

- Kontaktherstellung,

- Lernberatung,

- Personalberatung,

- Finanzierungsberatung,

- Forschungsrecherchen.

Mit dieser Ausrichtung kann die Arbeit der Einrichtungen für wissenschaftliche Weiterbildung auch in Rückbezug auf ihre eigene Geschichte fortgesetzt werden. Die Zentral-, Kontakt- und Transferstellen für wissenschaftliche Weiterbildung sind ursprünglich meist in den 1970er Jahren entstanden, als die Hochschulpolitik, ausgehend von demokratischen Impulsen, eine Öffnung des Hochschulzugangs und vor allem die Teilhabe an wissenschaftlichem Wissen für breitere Bevölkerungsgruppen intendierte. Wissenschaftliche Weiterbildung war Beitrag zur Studienreform und zur Demokratisierung der Hochschulen. Mit den neuen Herausforderungen hat sich diese Aufgabe noch verstärkt. 


\section{Empfehlungen zur Weiterentwicklung wissenschaftlicher Weiterbildung}

Der Stellenwert der Hochschulangebote in der wissenschaftlichen Weiterbildung entscheidet sich letztlich daran, inwieweit sie in der Lage sind die wachsende Nachfrage aufzugreifen. Im Vordergrund steht der Nutzen für die Lernenden: thematisch bezogen auf die Brauchbarkeit der Angebote, formal hinsichtlich der durch Zertifikate belegten Ansprüche.

Um solche Angebote wissenschaftlicher Weiterbildung zu entwickeln, müssen in den Hochschulen Ressourcen aktiviert bzw. akquiriert werden. Diese werden nur dann Zukunftsfähigkeit erhalten, wenn kontinuitätssichernde Organisationsformen institutionalisiert werden.

\subsection{Struktur der Angebote und Zertifikate}

Die Veranstaltungsformen der wissenschaftlichen Weiterbildung umfassen ein Spektrum, das von Einzelveranstaltungen über systematisierte Programme bis zu mehrsemestrigen weiterbildenden Studiengängen reicht. Es schließt auch Tagungen und Kongresse ein. Diese Angebotsvielfalt, welche auf spezifische Nachfrage reagiert, gilt es zu erhalten und gleichzeitig transparenter zu machen.

Diejenigen Weiterbildungsangebote der Hochschulen, die sich hauptsächlich an berufstätige Erwachsene wenden, sind entsprechend meist berufsbegleitend organisiert. Für die Lernenden liegt der Wert von Weiterbildung auf zwei Ebenen: Zum einen geht es um die inhaltliche Bedeutsamkeit der Themen für individuelle Verwendungszusammenhänge. Dabei kann ein Abschluss gleichgültig oder überflüssig sein. Zum andern wird nach dem formalen Nutzen der Zertifikate für Abschlüsse, Berechtigungen und deren Verwertungsmöglichkeiten gefragt.

Am Ende weiterbildender Studiengänge stehen in der Regel Zertifikate, deren Vergabe an Leistungsnachweise gebunden ist. Der Nutzen dieser Zertifikate für die Absolventen ist umso höher, je angesehener die anbietenden Institutionen sind. Im Rahmen der Gesamtentwicklung sind deshalb Qualitätssicherung, Zertifizierung und Akkreditierung im Zusammenhang zu sehen. Daraus ergeben sich Problemaspekte für abschlussbezogene Angebote wissenschaftlicher Weiterbildung als zu gestaltende Herausforderungen:

- Adressaten und Zugang: Wird der Zugang für Adressaten ohne Hochschulzugangsberechtigung bzw. -abschluss offengehalten?

- Angebotsumfang: Ist die Zeitbelastung für berufstätige Teilnehmende zumutbar?

- Studierbarkeit und Angebotsorganisation: Ist die organisatorische und zeitliche Angebotsform den Teilnahmemöglichkeiten angemessen?

- Trägerschaft/Anbieter: Bleibt die Verantwortung für die Angebote bei der Hochschule? 
- Lehrpersonal: Ist die Wissenschaftlichkeit der Lehre gesichert?

- Durchlässigkeit und Anrechenbarkeit: Werden vorgängige Erfahrungen und Leistungen angerechnet und sind die Lernerfolge mit entsprechenden Berechtigungen verbunden?

- Abschlussbezeichnungen und Wertigkeit: Bringen die Abschlüsse den Absolventen einen inhaltlichen und formalen Nutzen?

Zum weiterbildenden Studium können nach Gesetzeslage in den Bundesländern Hochschulabsolventen sowie Personen zugelassen werden, die die Eignung im Beruf oder auf andere Weise erworben haben. Eine Anerkennung vorgängigen Lernens (recogniton of prior learning) und eine Anrechnung nachgewiesener Kompetenzen findet in Ansätzen statt. Angesichts der Ablösung tatsächlicher beruflicher Kompetenz von früher erworbenen formalen Abschlüssen muss gesichert werden, dass wissenschaftliche Weiterbildung für interessierte, erfahrene und geeignete Berufstätige zugänglich bleibt.

Besonders die Frage nach Zertifikaten erhält wachsendes Gewicht. Mit den gestuften Studienabschlüssen des BA/MA-Konzepts entwickeln sich zunehmend auch Standards für Module - also auch unterhalb der Studienabschlüsse. Gleichzeitig ist die Vergabe von Zertifikaten in der wissenschaftlichen Weiterbildung noch uneinheitlich. Selbst innerhalb einzelner Einrichtungen der wissenschaftlichen Weiterbildung ist die Vergabe von Abschlussdokumenten nicht immer einheitlich geregelt; vergeben werden: Teilnahmebescheinigungen, Teilnahmezertifikate (mit Prüfungsleistungen), Abschlusszeugnisse, auch akademische Abschlüsse. Diese Uneinheitlichkeit schwächt das Profil der Weiterbildungsangebote. Gleichzeit ist es notwendig, die Abschlüsse auf die Gesamtstruktur der Studienangebote zu beziehen und zu vereinheitlichen.

Mit der Entwicklung verstärkter Abschlussbezogenheit für die wissenschaftliche Weiterbildung einerseits wächst andererseits die Gefahr einer mehrfachen Eingrenzung der Angebote durch Dominanz einer beruflichen Orientierung bei Intentionen, Themen und Adressaten. Demgegenüber gibt es auch eine starke Nachfrage nach nicht direkt verwertbaren Angeboten. Hierbei geht es vor allem um interdisziplinäres Zusammenhangswissen und fachgrenzenüberschreitendes Verstehen.

Die Lehrpersonen in der wissenschaftlichen Weiterbildung benötigen eine besondere didaktische Kompetenz und entsprechende Beratung, um sie auf die Besonderheiten des Studiums berufstätiger Erwachsener vorzubereiten. Dafür können die Organisationseinheiten für die wissenschaftliche Weiterbildung professionelle Unterstützungsleistungen bieten.

Zunehmend kommen im Angebot der Hochschulen E-Learning-Module und -Studiengänge zum Einsatz. Hier gibt es vielfältige und effektive Formen, von eigenständigen Angeboten über die Kooperation verschiedener Hochschulen bis zur internationalen Zusammenarbeit. Für den Einbezug von E-Learning-Konzepten in Systeme von Blended Learning müssen zusätzliche Kriterien festgelegt werden. 


\section{DGWF-Empfehlung}

Auf dem Weg der Modularisierung und der Eingliederung vorhandener Angebote in das ECTS sollte vorangeschritten werden.

Die DGWF empfiehlt die Entwicklung von Standards für die Vergabe von Zertifikaten. Diese sollten sich an den hochschulischen Anforderungen orientieren und nach dem Muster des „Diploma Supplement“ inhaltliche Schwerpunkte umfassen. Bei umfangreicheren Abschlusszertifikaten sollten darüber hinaus die Abschlussarbeit und ggf. erbrachte Teilleistungen aufgenommen werden.

Die Hochschulen werden aufgefordert, für weiterbildende Studiengänge mit akademischem Abschluss Zugänge auch für Teilnehmende ohne Hochschulabschluss weiter zu eröffnen und dafür notwendige Gesetzesänderungen anzuregen. Alternative Zugangswege sind zu regeln, die erforderlichen Kompetenzen sind in jeweils geeigneter Weise festzustellen.

Die Anerkennung vorgängigen Lernens und die Anrechnung vorhandener Kompetenzen sollen im Rahmen von Projekten erprobt werden. Die dabei zu gewinnenden Erkenntnisse können zum Maßstab für allgemeine Regeln der Hochschulen genommen werden.

Um die Breite des Angebotsspektrums entsprechend dem jeweiligen Hochschulprofil zu sichern, geht es um den Erhalt und den weiteren Ausbau

- eines breitbandigen Profils wissenschaftlicher Weiterbildung,

- heterogener Ziel- und Teilnahmegruppen,

- von umsetzungs- und anwendungsorientierter Fächervielfalt und Interdisziplinarität unter Berücksichtigung der Geistes- und Sozialwissenschaften,

- von Angeboten unterhalb von akkreditierten Angeboten.

\subsection{Qualität und Akkreditierung der Angebote}

Für die im Rahmen der Hochschulen vorhandenen Angebote wissenschaftlicher Weiterbildung gelten grundsätzlich die gleichen Qualitätsmaßstäbe wie für Angebote des grundständigen Studiums.

Daraus resultieren Anforderungen an ein systematisch aufgebautes Modell:

- Vergleichbarkeit sichern und spezifische Träger- und Maßnahmeentwicklungen zulassen,

- Transparenz herstellen und gleichzeitig regionale und spezifische Unterschiede gewährleisten,

- Selbst- und Fremdevaluation kombinieren und

- gleichzeitig den Aufwand einschränken, aber auch

- verlässliche Aussagen der Evaluation zulassen.

In fast allen Einrichtungen für wissenschaftliche Weiterbildung findet eine Evaluation von Bildungsveranstaltungen statt. In der Mehrzahl der Einrichtungen hat sich jedoch ein systematisches Qualitätsmanagement noch nicht durchgesetzt, welches 
sich auch auf weitere Aspekte als auf die Input-, Durchführungs- und Ergebnisqualität bezieht. Entwicklungspotenziale bestehen insbesondere bei

- der Qualitätspolitik,

- den Qualitätszielen,

- der Kompetenzverteilung und

- der Gewichtung der Qualitätssicherungspraktiken.

Neben den bisherigen Qualitätssicherungsverfahren (z. B. Verabschiedung von Programmen, Qualifikationsprüfungen bei Lehrbeauftragten durch akademische Gremien, Evaluation einzelner Maßnahmen) sind Qualitätssicherungssysteme erst in Ansätzen vorhanden. Es gibt Erfahrungen mit den in der Weiterbildung verbreiteten Qualitätssicherungssystemen EFQM und LQW. Gleichzeitig wird die wissenschaftliche Weiterbildung einbezogen in das hochschulspezifische Verfahren der Akkreditierung. Bachelor- und Masterstudiengänge, auch weiterbildende Bachelor- und Masterstudiengänge müssen akkreditiert werden.

\section{DGWF-Empfehlung}

Die Angebote der hochschulischen Weiterbildung in der wissenschaftlichen Weiterbildung unterliegen grundsätzlich den gleichen Qualitätskriterien wie im grundständigen Studium.

Ein Forschungsbezug ist anzustreben. Die wissenschaftliche Leitung der Programme obliegt in der Regel Professor/innen der Hochschule. Das Verhältnis zwischen Dozent/innen aus dem eigenen Haus und Lehrbeauftragten sollte der Thematik angemessen sein. Lehrende, die von außen kommen, müssen Voraussetzungen erfüllen, die denen für sonstige Lehrbeauftragte der Universität vergleichbar sind.

Alle Programme (Studiengänge, Kurse, Seminare, Module) der wissenschaftlichen Weiterbildung werden von akademischen Gremien beraten und verabschiedet. Prüfungsverfahren und Prüfungsdurchführung in weiterbildenden Zertifikats-, Bachelorund Masterstudiengängen entsprechen in Art und Weise vergleichbaren anderen Prüfungen an der Hochschule.

Teilnehmendenschutz im Sinne von „Verbraucherschutz“ ist ein zentrales Thema. Dies bezieht sich auf

- die Informationspolitik (Transparenz usw.),

- die Teilnahmeverträge und

- das Beschwerdeverfahren.

In Bezug auf den Teilnehmendenschutz wird eindringlich auf die Empfehlungen des Deutschen Instituts für Erwachsenenbildung und der Stiftung Warentest (Bildungstests) verwiesen.

Die DGWF empfiehlt, dass sich Einrichtungen der Weiterbildung zum einen an den Kriterien der Akkreditierungsverfahren orientieren. Zum anderen wird angeregt, dass Mitarbeiter/innen der Einrichtungen für Weiterbildung sich aktiv als Gutachter/innen an der Akkreditierung beteiligen. Die Beteiligung der wissenschaftlichen 
Weiterbildung an Akkreditierungsverfahren selbst kann diese für Belange der Einrichtungen für Weiterbildung öffnen und spezifizieren; demgemäß wird die Bildung von Gutachter/innen-Pools aus der wissenschaftlichen Weiterbildung angeregt.

Zusätzlich kann eine „systemnahe“ eigenständige Qualitätstestierung für die Aktivitäten der Weiterbildung sinnvoll sein (z. B. EFQM oder LQW).

\subsection{Kapazitäts- und Deputatswirksamkeit wissenschaftlicher Weiterbildung}

Bisher wird wissenschaftliche Weiterbildung sowohl in nicht zertifikatsorientierter als auch in abschlussbezogener Form (Zertifikate oder Weiterbildungsmaster und auch Weiterbildungsbachelor) - was die Art der Lehrverpflichtung hochschulischer Lehrpersonen anbetrifft - überwiegend in Nebentätigkeit durchgeführt.

Die Anrechnung auf das Deputat ist in vielen Bundesländern und Hochschulen möglich, wenn der Fachbereich bzw. die Fakultät dafür Kapazitäten zur Verfügung stellt, was sich allerdings bei Zugangsbeschränkungen mindernd auf die Zulassungszahlen des erstausbildenden Studiums auswirkt. Es sind auch Ausgleichsregelungen möglich, wonach bei Deputatsanrechnung der Fachbereich bzw. die Fakultät Mittel für Lehraufträge (aus den Einnahmen) erhält, um eventuelle Ausfälle im grundständigen Studium auszugleichen.

Die Lehraufgaben der Hochschulen werden von den Wissenschaftlerinnen und Wissenschaftlern der Fachbereiche bzw. Fakultäten wahrgenommen. Angesichts der Tatsache, dass mit der Einführung der gestuften Studienabschlüsse auch ein Masterabschluss im weiterbildenden Studium möglich geworden ist, lässt sich kaum noch legitimieren, dass ein Teil der Lehre, nämlich die weiterbildende Lehre, obwohl zu den Kernaufgaben der Hochschule gehörend, in Nebentätigkeit ausgeführt wird. Eine Rechtfertigung dafür war nur gegeben, solange die Anrechnung auf das Deputat bei Lehre in nicht abschlussbezogener Weiterbildung zu einer Minderung der Aufnahmekapazitäten beim ausbildenden Studium führte.

\section{DGWF-Empfehlung}

Die Hochschule sollte bekräftigen, dass Lehre in der Weiterbildung auf das Deputat angerechnet werden kann. Dabei kann in der Regel eine Minderung der Kapazitäten beim abschlussbezogenen Studium durch Lehraufträge, welche aus den Einnahmen der Weiterbildung zu finanzieren sind, verhindert werden.

Für weiterbildende Bachelor- bzw. Masterstudiengänge sollte eine kapazitäre Verankerung im Spektrum abschlussbezogener Studiengänge eines Fachbereichs bzw. einer Fakultät mindestens nach der Erprobungs- und Einführungsphase angestrebt und umgesetzt werden.

Durch die Kapazitätswirksamkeit weiterbildender Bachelor- und Masterstudiengänge wird es möglich, Lehrpersonen im Rahmen ihres Deputats in weiterbildenden Studiengängen einzusetzen. Dies sollte insbesondere bei „Kern-Lehrpersonen“ greifen. 


\subsection{Kosten und Finanzierung wissenschaftlicher Weiterbildung}

Die rechtlichen Vorgaben der Bundesländer legen in der Regel fest, dass für das weiterbildende Studium mindestens kostendeckende Gebühren zu erheben sind.

Die Hochschulen sind derzeit in vielen Fällen nicht in der Lage, die Kosten einzelner Angebote zu beziffern. Außerdem lassen es eine Reihe von Faktoren nicht sinnvoll erscheinen, das Gebot der Kostendeckung auf einzelne Angebote zu beziehen. $\mathrm{Zu}$ den Kostenfaktoren gehören z. B.

- Entwicklungskosten,

- Markteinführung,

- Unterdeckung durch Abmeldungen oder durch nicht ausreichend belegte Module.

Dazu kommt die wissenschaftliche oder gesellschaftliche Relevanz von Angeboten, die nicht marktgängig sind. Es ergibt darum Sinn, das Gebot der Kostendeckung grundsätzlich auf die Durchführungskosten der Angebote insgesamt zu beziehen und im Einzelfall sogar Unterdeckungen in Kauf zu nehmen.

Die Kosten wissenschaftlicher Weiterbildung werden durch Mittel aus dem Wirtschaftsplan der Hochschule, durch Drittmittel, größtenteils aber durch Teilnehmerentgelte finanziert. Letztere werden zum Teil auf der Grundlage staatlicher Gebührenordnungen für das Hochschulwesen, zum Teil auf der Grundlage hochschuleigener Gebührenordnungen erhoben. Grundlage für Letztere stellen in der Regel auch Kostenkalkulationen dar, die aufgrund der Buchhaltung der Hochschulen jedoch oft nur mit pauschalierten Größen arbeiten können.

Die Vergütungen, die für Lehrleistungen in der wissenschaftlichen Weiterbildung gezahlt werden, richten sich zum Teil nach den Sätzen für Lehrbeauftragte der Hochschulen und zum Teil nach Sätzen, von denen angenommen wird, sie seien im jeweiligen Zusammenhang marktüblich. Letzteres gilt auch für andere Leistungen in der wissenschaftlichen Weiterbildung.

Eine geordnete Verteilung der Einnahmen aus wissenschaftlicher Weiterbildung nach Abzug aller Kosten findet bisher nicht statt.

\section{DGWF-Empfehlung}

Die Hochschulen streben durch Zielvereinbarung mit der jeweiligen Landesregierung an, das Gebot der Kostendeckung auf die Durchführungskosten und auf die Gesamtheit aller Angebote der wissenschaftlichen Weiterbildung zu beziehen, damit auch zukünftig wirtschaftliches Handeln möglich bleibt.

Die Festsetzung der Teilnehmerentgelte soll sich grundsätzlich an einer Kostenkalkulation (kaufmännische Buchhaltung) ausrichten, wobei Abweichungen nach oben und unten möglich bleiben sollten, solange das Gebot der Kostendeckung in der Ausprägung nicht infrage steht. 
Soweit Lehrleistungen vergütet werden, sollte der Lehrauftragssatz der Hochschulen die Richtschnur bilden. Höhere Vergütungen sind möglich, wenn Markterfordernisse es geraten erscheinen lassen und Landesmittel nicht oder nur im geringen Maße in die Finanzierung einfließen.

Die Hochschule regelt die Höhe der Vergütungen in einer eigenen Honorarordnung für die wissenschaftliche Weiterbildung, ohne dass dabei die notwendige Flexibilität im Einzelfall eingeschränkt wird.

Aus Teilnahmeentgelten, die nicht zur Deckung der Durchführungskosten benötigt werden, sind Beiträge zur Deckung der Fixkosten zu leisten.

Die Hochschule richtet einen zentralen Fonds „Wissenschaftliche Weiterbildung“ ein, aus dem die Entwicklung neuer Angebote, die Markteinführung neuer Angebote, temporäre Defizite, Investitionen u. a.m. finanziert werden können. Der Fonds wird nach einer angemessenen Ersteinlage durch die Hochschule aus Weiterbildungseinnahmen gespeist. Die dafür abzuführenden Mittel sollen in einem angemessenen Verhältnis stehen zu den Mitteln, die bei der veranstaltenden Stelle verbleiben.

\subsection{Organisation der Weiterbildung}

Unter dem Dach einer Hochschule bestehen häufig mehrere Einrichtungen, die sich - größtenteils neben anderen Aufgaben - koordinierend um die Entwicklung von Transferaktivitäten und das Angebot weiterbildender Veranstaltungen kümmern, z. B. zentrale Einrichtungen für Weiterbildung, Technologietransfer-Einrichtungen, Service-Büros der Verwaltung usw. Hinzu kommen die Lehreinheiten der Fakultäten, die Angebote der wissenschaftlichen Weiterbildung entwickeln und durchführen, Einrichtungen des Studium Generale sowie An-Institute, Freundeskreise, Hochschulgesellschaften usw., die ebenfalls in diesem Feld tätig werden.

Diese gewachsene Gemengelage ist weder nach innen noch nach außen begründbar und verständlich zu machen. Nach innen nicht, weil es nicht den Zufällen von Kontakten und Kenntnissen überlassen bleiben darf, welche Einrichtung oder Ebene in welcher Weise mit einem Weiterbildungsvorhaben befasst wird. Nach außen deshalb nicht, weil sie weder kundenfreundlich noch kooperationsoffen ist. Außerdem spricht vieles dafür, dass durch die zergliederte Organisationsstruktur Ressourcen und Synergieeffekte nicht oder nicht hinreichend genutzt werden.

\section{DGWF-Empfehlung}

Die Einrichtungen werden in einer gemeinsamen zentralen Institution zusammengefasst. $\mathrm{Zu}$ prüfen ist die Einbeziehung ausgelagerter Einrichtungen, die von der Hochschule gesteuert werden. In einem ersten Schritt sollen vorhandene Profile erhalten und gegebene Rechtsformen weitergeführt werden, soweit sie sich bewährt haben. 
Die Aufgaben der zentralen Institution liegen in der Steuerung aller Weiterbildungsprozesse der beteiligten Einrichtungen, der Zusammenführung und Optimierung von Querschnittsaufgaben wie Management, Marketing, didaktische Entwicklung und Unterstützung sowie der gemeinsamen Nutzung von Räumen, Geräten und Material.

In den akademischen Angelegenheiten der zentralen Institution entscheidet ein Gremium unter der Beteiligung des für die Weiterbildung zuständigen Mitglieds des Präsidiums bzw. des Rektorats. Das Gremium verhandelt Ziel- und Leistungsvereinbarungen mit dem Präsidium.

In einem zweiten Schritt sind die Profile und die gegebenen Rechtsformen zu prüfen. Darüber hinaus sollte ein gemeinsames Weiterbildungsbudget verhandelt und verteilt werden.

Mit Sitz und Stimme sind in diesem Weiterbildungsgremium der Hochschule vertreten:

- die Leitungen der zentralen Einrichtungen und ggf. der Verwaltungs- und weiterer Einheiten,

- die Leitungen von Instituten, die eigenständig wissenschaftliche Weiterbildung anbieten,

- die Leitungen von An-Instituten entsprechender Geschäftsfelder, soweit die Hochschulbeteiligung hier maßgeblich ist,

- die Studiendekane der Fakultäten bzw. der analogen wissenschaftlichen Einheiten, sofern vorhanden die geschäftsführende Leitung von Instituten für Weiterbildung, Erwachsenenbildung bzw. Personalentwicklung.

Es ist zu prüfen, ob diesem Gremium ein Beirat zugeordnet wird. Dieser Beirat ist zu besetzen mit regional bedeutsamen Vertreter/innen aus Wirtschaft, Kommune (Region) und Verbänden. Der Beirat unterstützt die wissenschaftliche Weiterbildung bei der strategischen Entwicklung der Geschäftsfelder und der Angebote.

Bei einem hohen Aktivitätsniveau und -spektrum ist es sinnvoll für thematisch gebündelte Angebote weitere Gremien einzusetzen, die die Funktionen von Fachbeiräten übernehmen.

Das zentrale Gremium für die Weiterbildung

- prüft die Einbindung der wissenschaftlichen Weiterbildung in das Leitbild der Hochschule und

- entwirft das Weiterbildungskonzept für die Hochschule bzw. entwickelt es weiter; das Weiterbildungskonzept wird von den zuständigen Entscheidungsgremien der akademischen Selbstverwaltung der Hochschule, der Hochschulleitung und ggf. eingesetzten Hochschulräten diskutiert und verabschiedet.

Je nach Hochschulstandort und Umfang des Angebots an wissenschaftlicher Weiterbildung kann sich für die Zukunft auch empfehlen, die Bündelung der Weiterbildung in einer eigenständigen Fakultät zu prüfen. Doppelmitgliedschaften der Fächer bieten dafür die Grundlage. 


\subsection{Stärkung des wissenschaftlichen Profils}

Bei einer sich zuspitzenden, nach außen gerichteten wissenschaftlichen Profilierung der Hochschulen, wie sie in der Diskussion um Exzellenz forciert wird, müssen die Einrichtungen für wissenschaftliche Weiterbildung selbst ihre Wissenschaftlichkeit nachweisen.

Die vorrangige Bezugsdisziplin der wissenschaftlichen Weiterbildung - die Weiterbildungswissenschaft - hat sich bisher nicht in einem Ausmaß profiliert, um sich im Wettstreit um „Exzellenz“ weit genug vorn zu platzieren. Die Einrichtungen müssen selbst dazu beitragen, wenn es darum geht, die bildungswissenschaftlichen Aufgaben mit ökonomischen Gegebenheiten produktiv zu verbinden und zu gestalten.

\section{DGWF-Empfehlung}

Zur Stärkung der Einrichtungen für Weiterbildung an Hochschulen ist eine wissenschaftliche Leitung sowie die Unterstützung und Beratung durch einen entsprechend profilierten Beirat sicherzustellen.

In den Arbeitsverträgen für das beschäftigte Personal ist ein angemessenes Verhältnis von wissenschaftlichen und Dienstleistungstätigkeiten darzustellen. Dabei ist es unerheblich, ob die Beschäftigten arbeitsrechtlich in der Gruppe der „Wissenschaftler/innen" (Fakultäten, wissenschaftliche Betriebseinheiten) oder in der Gruppe "technischer und Verwaltungsdienst" geführt werden. Die wissenschaftliche Grundlegung der Tätigkeit ist im Profil und im Leitbild der Einrichtung deutlich auszuweisen.

Im Mittelpunkt der wissenschaftlichen Tätigkeit steht auf konzeptioneller Ebene ein anwendungs- und umsetzungsbezogener Ansatz der wissenschaftlichen Begleitforschung.

Zur Unterstützung des wissenschaftlichen Profils ist eine verstärkte Teilnahme an entsprechenden Modellversuchen, Projekteinwerbungen und wissenschaftlichen Wettbewerben zu realisieren. 



\section{Verzeichnis der Autorinnen und Autoren}

\section{Ursula Bade-Becker, Dr.}

Geschäftsführerin des Zentrums für wissenschaftliche Weiterbildung an der Universität Bielefeld e. V. Stellv. Leiterin der Kontaktstelle Wissenschaftliche Weiterbildung der Universität Bielefeld. Stellv. Vorsitzende der Deutschen Gesellschaft für wissenschaftliche Weiterbildung und Fernstudium e. V. von 2012-2016. Sprecherin der Arbeitsgruppe der Einrichtungen für Weiterbildung an Hochschulen (AG-E) in der DGWF von 2008-2012.

ursula.bade-becker@uni-bielefeld.de

\section{Sybille Barth, Dipl.-Ing. oec.}

Friedrich-Alexander-Universität Erlangen-Nürnberg (FAU) Referat F2, Leiterin der Kontaktstelle für Wissens- und Technologietransfer und der wissenschaftlichen Weiterbildung; Projektkoordination der Projekte STRUKTUR 2013 und STRUKTUR 2015 .

sybille.barth@fau.de

\section{Thomas Bertram, Dipl.-Päd.}

Leibniz Universität Hannover, Zentrale Einrichtung für Weiterbildung, Leitung Gasthörenden- und Seniorenstudium, Vorsitzender des Sprecher/innen/rates der BAG WiWA seit 2016. www.ghs.uni-hannover.de

Thomas.Bertram@zew.uni-hannover.de

\section{Bernhard Christmann, Dipl.-Ing.}

bis März 2012 Geschäftsführer der Akademie der Ruhr-Universität gGmbH, Bochum. Von 1994 bis 2008 Vorsitzender des Sprecherrates der AG-E; von 2003 bis 2012 Mitglied im Vorstand der DGWF.

bernhard.christmann@online.de 


\section{Silvia Dabo-Cruz, Dipl.-Päd.}

Universität des 3. Lebensalters an der Goethe-Universität Frankfurt am Main, Leiterin der Geschäftsstelle, Vorsitzende des Sprecher/innen/rates der BAG WiWA von 2011 bis 2016. www.uzl.uni-frankfurt.de

dabo-cruz@em.uni-frankfurt.de

\section{Sylvia Derra, M.A.}

Friedrich-Alexander-Universität Erlangen-Nürnberg (FAU) Referat L1 - Rechtsangelegenheiten, Studienprogrammentwicklung und Studienzuschüsse, Konzeptionierung und Einrichtung von weiterbildenden und berufsbegleitenden Studiengängen.

sylvia.derra@fau.de

\section{Andreas Fischer, Dr.}

Direktor des Zentrums für universitäre Weiterbildung der Universität Bern, Gründungsmitglied von Swissuni. Arbeitsschwerpunkte: Wissenschaftliche Weiterbildung, Programmentwicklung, Weiterbildungsmanagement sowie Weiterbildungspolitik.

andreas.fischer@zuw.unibe.ch

\section{Stefan Gesmann, Prof. Dr.}

FH Münster, Fachbereich Sozialwesen, Geschäftsführer Referat Weiterbildung, Lehrund Forschungsgebiet: Erwachsenenbildung/Weiterbildung in der Sozialen Arbeit.

s.gesmann@fh-muenster.de

\section{Elke Gornik, Mag. MBA}

Deputy Director des Postgraduate Center der Universität Wien, Leiterin der Bereiche Postgraduate Programs und Qualitätsmanagement, AUCEN-Vorstandsmitglied seit 01/2016; Veröffentlichungen im Bereich Lifelong Learning und wissenschaftliche Weiterbildung.

elke.gornik@univie.ac.at 


\section{Beate Hörr, Dr. phil., M.A.}

Leiterin des Zentrums für wissenschaftliche Weiterbildung an der Johannes Gutenberg-Universität Mainz (JGU).

2012-2016 Vorsitzende der Deutschen Gesellschaft für wissenschaftliche Weiterbildung und Fernstudium e.V. (DGWF), seit 2016 Beisitzerin („Internationales“). 2010-2015 Mitglied im Steering Committee EUCEN (European Universities Continuing Education Network).

hoerr@zww.uni-mainz.de

\section{Wolfgang Jütte, Prof. Dr.}

Professor für Weiterbildung an der Fakultät für Erziehungswissenschaft der Universität Bielefeld; Sprecher der AG Forschung der DGWF und geschäftsführender Herausgeber der Zeitschrift „Hochschule und Weiterbildung“ (zhwb). Arbeitsschwerpunkte: Wissenschaftliche Weiterbildung, Kooperations- und Netzwerkforschung, internationale Erwachsenenbildung, pädagogische Professionalität.

wolfgang.juette@uni-bielefeld.de

\section{Karla Kamps-Haller, Dipl.-Hdl.}

bis Dezember 2015 Direktorin des iwib - Institut Weiterbildung im Beruf der Hochschule RheinMain in Wiesbaden. Seit Januar 2016 Training und Beratung, Schmitten im Taunus.

karla.haller@t-online.de

\section{Peer-Olaf Kalis, Dipl.-Ing.}

Projektleiter Wissenschaftliche Weiterbildung in der Zentraleinrichtung wissenschaftliche Weiterbildung und Kooperation der Technischen Universität Berlin, Arbeitsschwerpunkt derzeit Lehren und Lernen mit digitalen Medien, Vorsitzender des Sprecherrats der DGWF Berlin/Brandenburg und Vorstandsmitglied der DGWF, Beiratsmitglied des Berliner Zentrums für Hochschullehre und im Projekt „Smart Learning“ der Handwerkskammer Berlin.

peer-olaf.kalis@tu-berlin.de

\section{Bernd Kaßebaum, Dr.}

IG Metall Vorstand, Ressort Bildungs- und Qualifizierungspolitik bis Ende 2016. Mitglied im Wissenschaftlichen Beraterkreis von ver.di und IG Metall.

bernd.kassebaum@igmetall.de 


\section{Monika Kil, Prof. Dr.}

Vize-Rektorin Lehre/Wissenschaftliche Weiterbildung, Departmentleiterin und Professur Weiterbildungsforschung und Bildungsmanagement an der Donau-Universität Krems, AUCEN-Vorstandsmitglied seit 2016, QM für die Wissenschaftliche Weiterbildung, Berufsbegleitendes Studieren, insbes. Blended Learning.

monika.kil@donau-uni.ac.at

\section{Margot Klinkner, Dr.}

Stellvertretende Geschäftsführerin Zentralstelle für Fernstudien an Fachhochschulen - ZFH, Koblenz.

Leitung Bereich II: Einrichtung, Vermarktung und Qualitätssicherung von Studiengängen und Studienmaterialien.

m.klinkner@zfh.de

\section{Maria Kondratjuk, M.Sc. Bildungsmanagement/Dipl.-Sozialpädagogin}

Wissenschaftliche Mitarbeiterin an der Professur für Erwachsenenbildung/Weiterbildung und Medienpädagogik der Universität Potsdam und wissenschaftliche Mitarbeiterin im Arbeitsbereich fokus: LEHRE an der Professur für Hochschulforschung und Professionalisierung der akademischen Lehre der Otto-von-Guericke-Universität Magdeburg.

maria.kondratjuk@ovgu.de

\section{Burkhard Lehmann, Dr. phil.}

Geschäftsführer des Zentrums für Fernstudien und Universitäre Weiterbildung an der Universität Koblenz-Landau. Vorsitzender der Deutschen Gesellschaft für wissenschaftliche Weiterbildung und Fernstudium e. V. Arbeitsschwerpunkte: Wissenschaftliche Weiterbildung, Lehren und Lernen mit digitalen Bildungsmedien, Fernstudium. Autor von Fachartikeln und Fachgutachter.

lehmann@uni-koblenz.de

\section{Claudia Lobe, Dr.}

Wissenschaftliche Mitarbeiterin an der Universität Bielefeld im Bereich „Weiterbildung und Governance of Lifelong Learning“ an der Fakultät für Erziehungswissenschaft. Redaktionsmitglied der Zeitschrift Hochschule und Weiterbildung (ZHWB). Forschungsfelder: Teilnehmerforschung, Biografie und Lebenslauf, pädagogische Hochschulforschung.

claudia.lobe@uni-bielefeld.de 


\section{Katharina Mallich-Pötz, Mag. Dr.}

Leiterin Personalentwicklung an der Medizinischen Universität Wien seit 2010, AUCEN-Geschäftsstellenleiterin seit 2016; Strategische Planung sowie Umsetzung von Personalentwicklungsmaßnahmen (z. B. Diversity Management, Konfliktkultur, Leadership) an der MedUni Wien, universitätsübergreifende Vernetzungsaktivitäten im Rahmen von AUCEN.

katharina.mallich-poetz@meduniwien.ac.at

\section{Annekatrin Mordhorst}

Leiterin der Stabsstelle für wissenschaftliche Weiterbildung der Christian-AlbrechtsUniversität zu Kiel. Schwerpunkte der Tätigkeit: Fortbildung für das Hochschulpersonal allgemein; Hochschuldidaktik; Fortbildung für das wissenschaftliche Hochschulpersonal; Weiterbildungs-Beratung; Coaching; Career Center; Wissenschaftliche Weiterbildung.

amordhorst@uv.uni-kiel.de

\section{Karin Pauls}

Universität Hamburg, Arbeitsstelle für wissenschaftliche Weiterbildung, Wissenschaftliche Mitarbeiterin, Mitglied im Sprecher/innen/rat der BAG WiWA seit 2011. k.pauls@aww.uni-hamburg.de,www.aww.uni-hamburg.de

\section{Michael Renz, M.A.}

Geschäftsführer Campus-Akademie für Weiterbildung der Universität Bayreuth, Projektleitung externe Weiterbildung, wissenschaftlicher Mitarbeiter im BMBF-Projekt "QuoRO“.

michael.renz@uni-bayreuth.de

\section{Sabine Riemer, Dipl.-Päd.}

Wissenschaftliche Mitarbeiterin und Lehrende in der Koordinierungsstelle für Weiterbildung der Hochschule Bremen. Schwerpunkte: Hochschuldidaktik, Kompetenzanalyse und Lernbiografieberatung.

sabine.riemer@hs-bremen.de 


\section{Johannes Ries, M.A.}

Friedrich-Alexander-Universität Erlangen-Nürnberg (FAU) Referat F2

Kontaktstelle für Wissens- und Technologietransfer - Bereich Wissenschaftliche Weiterbildung; Wissenschaftlicher Mitarbeiter in den Projekten STRUKTUR 2013 und STRUKTUR 2015 .

johannes.ries@fau.de

\section{Ortfried Schäffter, em. Prof. Dr.}

em. Professor für Theorie der Weiterbildung an der Humboldt-Universität zu Berlin. Zurzeit Mitarbeit am dreijährigen Projekt: „Berufsbiographische Übergangszeiten als Bildungsraum nutzen“. Das praxisfeldintegrierte Forschungsprojekt orientiert sich in seinem Format wissenschaftstheoretisch an einem Ansatz organisationsgebundener Professionalitätsentwicklung im „Mode 2“.

ortfried.schaeffter@googlemail.com

\section{Mandy Schulze, M.A., Dipl.-Betriebswirtin}

Wissenschaftliche Mitarbeiterin im Arbeitsbereich fokus: LEHRE an der Professur für Hochschulforschung und Professionalisierung der akademischen Lehre der Otto-von-Guericke-Universität Magdeburg, Doktorandin an der Humboldt-Universität zu Berlin und Lehrbeauftragte an der Alice-Salomon-Hochschule Berlin.

schulze_m@yahoo.de

\section{Wolfgang Seitter, Prof. Dr.}

Professor für Erwachsenenbildung/Weiterbildung an der Philipps-Universität Marburg, Weiterbildungsbeauftragter der Universität, wissenschaftliche Leitung des Marburger Teilprojektes von WM33 Weiterbildung Mittelhessen (2011-2017).

seitter@uni-marburg.de

\section{Anna Steiger, Mag.}

Vizerektorin für Personal und Gender, Technische Universität Wien; AUCEN- Vorstandsmitglied seit dem Gründungsjahr 2006, AUCEN-Vizesprecherin 2008 - 2015, AUCEN-Sprecherin seit 2016; Strategische Steuerung von Personaladministration, -entwicklung und -planung sowie des Bereichs Gender und Diversity an der TU Wien.

anna.steiger@tuwien.ac.at 


\section{Volker Stieg, Dipl.-Kfm.}

Cluster-Manager am Institut für Akademische Weiterbildung der Technischen Hochschule Ingolstadt.

volker.stieg@thi.de

\section{Christine Stöckler-Penz, Mag.}

Leiterin der Organisationseinheit „Life Long Learning“ an der Technischen Universität Graz, AUCEN-Vorstandsmitglied seit 2012, AUCEN-Vizesprecherin seit 2016; verantwortlich für die Gesamtkonzeption und -koordination der Weiterbildungsaktivitäten an der TU Graz in Zusammenarbeit mit den Instituten, Programmentwicklung, strategische Ausrichtung, Koordination der Programmabwicklung.

stoeckler-penz@tugraz.at

\section{Anno Stockem}

Geschäftsführer des Centers für lebenslanges Lernen $\left(\mathrm{C}_{3} \mathrm{~L}\right)$ der Universität Oldenburg. Er ist seit 1989 in unterschiedlichen Bereichen der Bildungsbranche tätig, u.a. im Klett-Konzern sowie in der Cornelsen Bildungsgruppe. Arbeitsschwerpunkte sind u. a. Bildungsmarketing sowie Management in Bildungsorganisationen.

anno.stockem@uni-oldenburg.de

\section{Annette Strauß, Dr.}

Geschäftsführerin der Agentur für wissenschaftliche Weiterbildung und Wissenstransfer - AWW e. V., Technische Hochschule Brandenburg, Beisitzerin im Vorstand der DGWF e. V., Mitglied im Sprecherrat der Landesgruppe Berlin-Brandenburg und der AG-F, Mitglied des Arbeitskreises wissenschaftliche Weiterbildung des Ministeriums für Wissenschaft, Forschung und Kultur (MWFK) des Landes Brandenburg.

annette.strauss@aww-brandenburg.de

\section{Kerstin Tänzer, M.A.}

Wissenschaftliche Mitarbeiterin für Weiterbildungsforschung, Weiterbildungsberatung und Netzwerkkommunikation im Zentrum für wissenschaftliche Weiterbildung (ZWW) der Hochschule Magdeburg-Stendal.

kerstin.taenzer@hs-magdeburg.de 
Silke Vergara, Dr. rer. pol.

Qualitätsmanagement und Leitung Studiengangsmanagement der berufsbegleitenden Masterstudiengänge der UNIKIMS - Management School der Universität Kassel. Sprecherin der Landesgruppe Hessen und stellvertretende Vorsitzende der Deutschen Gesellschaft für wissenschaftliche Weiterbildung und Fernstudium e.V.

vergara@uni-kassel.de

\section{Michael Vesper, Dipl.-Päd.}

Christian-Albrechts-Universität zu Kiel, Allgemeine Wissenschaftliche Bildungsangebote, Leiter der Geschäftsstelle, Mitglied im Sprecher/innen/rat der BAG WiWA seit 2007 .

mvesper@uv.uni-kiel.de

\section{Gabriele Vierzigmann, Prof. Dr. phil.}

Vizepräsidentin der Hochschule für angewandte Wissenschaften München (20082016), Ressort Weiterbildung und berufsbegleitendes Studium, wissenschaftliche Leiterin des BMBF-Projekts „Offene Hochschule Oberbayern“ (Teilprojekt München), Sprecherin der Landesgruppe Bayern und stellvertretende Vorsitzende der Deutschen Gesellschaft für wissenschaftliche Weiterbildung und Fernstudium e.V.

gabriele.vierzigmann@hm.edu

\section{Françoise de Viron, Prof., PhD}

President of eucen, European University Continuing Education Network, Professor at Université catholique de Louvain, GIRSEF/RIFA (Belgium).

francoise.deviron@uclouvain.be

\section{Helmut Vogt, Akademischer Direktor i. R.}

33 Jahre lang bis Ende März 2016 Leiter der Arbeitsstelle für wissenschaftliche Weiterbildung (AWW) der Universität Hamburg, von 1994 bis 2014 im Vorstand der DGWF, umfangreiche nationale und internationale Tätigkeiten, u.a. im Verwaltungsrat des Deutschen Instituts für Erwachsenenbildung, Bonn, und im Vorstand des European University Continuing Education Network (EUCEN), Barcelona.

helmut.vogt@uni-hamburg.de 


\section{Ulrich Wacker, Dr.}

Referent für Lebenslanges Lernen, Universität Konstanz, Geschäftsführer der Akademie für Wissenschaftliche Weiterbildung an der Universität Konstanz, Sprecher der Landesgruppe Baden-Württemberg, Mitglied im Sprecherrat der AG-E.

Ulrich.Wacker@uni-konstanz.de

\section{Markus Walber, Akad.-Dir. Dr.}

Leiter der Kontaktstelle Wissenschaftliche Weiterbildung und Rektoratsbeauftragter für wissenschaftliche Weiterbildung der Universität Bielefeld; Mitglied der Fakultät für Erziehungswissenschaft in der Arbeitsgruppe Weiterbildung \& Governance of Lifelong Learning.

markus.walber@uni-bielefeld.de

\section{Andrä Wolter, Prof. Dr.}

Professor für Erziehungswissenschaftliche Forschung zum Tertiären Bildungsbereich, Humboldt-Universität zu Berlin; 2012-2016 stellvertretender Vorsitzender der DGWF; Mitglied der Autorengruppe des Nationalen Bildungsberichts.

andrae.wolter@hu-berlin.de 


\section{methoden-kartothek.de}

Seminare online planen

\section{$\nearrow$ www.methoden-kartothek.de}

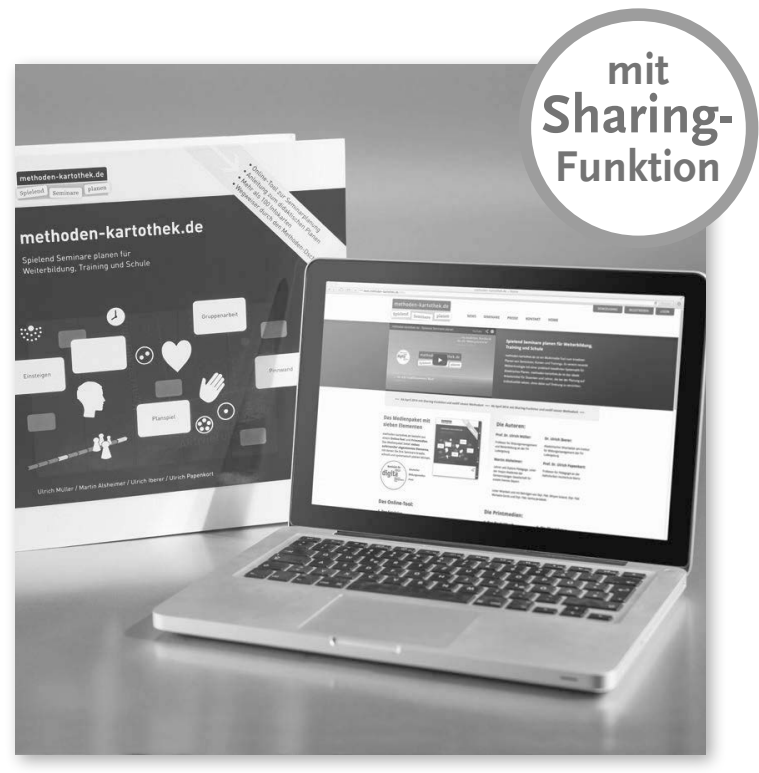

Einfache Seminarplanung: Ablaufpläne per Drag-anddrop erstellen

\section{Parametergesteuerte}

Methodenauswahl: Per Mausklick zur besten Methode für jede Seminarsituation

\section{Sharing-Funktion: Bearbeiten} von Seminaren im Team methoden-kartothek.de bietet einen schnellen und einfachen Zugang zu mehr als 150 Lehrmethoden für Seminare, Trainings oder Schulstunden.

Mit diesem Methodenkanon können Lehrende, Coaches und Trainer:innen Unterrichtseinheiten, Schulungen und Seminare planen, dokumentieren und archivieren.

\section{"Ein großer Wurf."}

Sascha Rex, dis.kurs (DVV)

\section{methoden-kartothek.de BASIS}

- Online-Tool inklusive Support und Update-Service - Didaktischer Leitfaden „Einführung in die Seminarplanung", $160 \mathrm{~S}$.

$69,00 €(D)$, Best.-Nr. 6004255

ISBN 978-3-7639-4984-7

\section{methoden-kartothek.de PREMIUM}

- Online-Tool inklusive Support und Update-Service - Didaktischer Leitfaden „Einführung in die Seminarplanung", 160 S.

- Ordner mit ausführlichen Methodenbeschreibungen $99,00 €(D)$, Best.-Nr. 6004249

ISBN 978-3-7639-4985-4

Ausführliche Informationen und den kostenlosen Demozugang erhalten Sie auf methoden-kartothek.de 

45 Jahre Deutsche Gesellschaft für wissenschaftliche Weiterbildung und Fernstudium - Zeit für eine Zwischenbilanz.

Mehr als 30 Autor:innen beleuchten in diesem Sammelband die Geschichte der Fachgesellschaft und bilanzieren die bisherige Arbeit der 12 Arbeitsgemeinschaften und Landesgruppen.

Die Beiträge geben Einblick in die thematisch ausdifferenzierte Verbandsarbeit sowie einen Überblick zum Stand der wissenschaftlichen Weiterbildung an Hochschulen. Darüber hinaus werfen die Autor:innen einen Blick auf die nahe Zukunft der noch relativ jungen fachwissenschaftlichen Disziplin und die weitere Professionalisierung des Feldes.

Doch der vorliegende Band möchte nicht nur informieren:

Die Autor:innen eröffnen Möglichkeiten des Engagements und laden Interessierte zur Mitarbeit ein. 